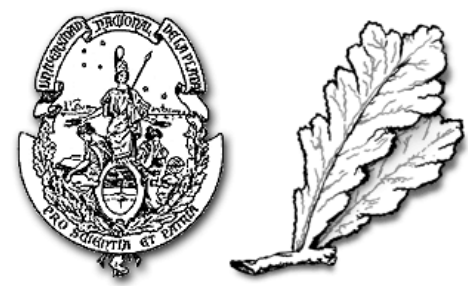

UNIVERSIDAD NACIONAL DE LA PLATA

FACULTAD DE INGENIERÍA

Departamento de Electrotecnia

\title{
Avances en Espectroscopia Mössbauer: Nuevas Estrategias para Experimentos de Velocidad Programable
}

\author{
Alejandro L. Veiga \\ TESIS PRESENTADA PARA EL GRADO DE DOCTOR EN INGENIERIA DE LA \\ UNIVERSIDAD NACIONAL DE LA PLATA
}

DIRECTOR: Dr. Miguel A. Mayosky.

CO-DIRECTOR: Ing. Nolberto Martínez.

INFRAESTRUCTURA PARA LA REALIZACION: Laboratorio de Electrónica Industrial, Control e Instrumentación (LEICI), Facultad de Ingeniería, UNLP, y Laboratorio de Espectroscopia Nuclear e Interacciones Hiperfinas (LENIH) del Departamento de Física de la UNLP, perteneciente al Instituto de Física La Plata (IFLP-CONICET). 



\section{Resumen}

La técnica Mössbauer es una herramienta fundamental para el estudio de la estructura nanoscópica de diversos materiales que representan un rol clave en el desarrollo tecnológico, especialmente el hierro y sus compuestos. La técnica de medición fue desarrollada en la década del 70, período en que se diseñó y perfeccionó el instrumental que actualmente se utiliza en la mayoría de los laboratorios. Desde entonces son pocas las modificaciones que se han realizado a la instrumentación asociada con este tipo de observación nuclear.

Las técnicas Mössbauer tradicionales de aceleración constante y de velocidad constante encuentran limitaciones en algunas experiencias particulares, sobre todo en lo que se refiere al estudio de la dinámica de los materiales ante variaciones de parámetros externos, como temperatura, campo magnético, presión o grado de evolución de una transición de fase.

En esta tesis se introduce una nueva técnica de medición, llamada de velocidad programable, que es una combinación de las dos técnicas mencionadas. Se realiza la especificación, diseño e implementación de la instrumentación necesaria para aplicar la nueva metodología en un laboratorio de espectroscopia nuclear tradicional. Se incluyen varios aspectos originales relacionados con la optimización de la detección de eventos resonantes y con la operación de los transductores a velocidad constante.

El nuevo modo de operación presenta ventajas relevantes respecto de los anteriores, principalmente en lo que se refiere a eficiencia y tiempo de adquisición, manteniendo la exactitud y precisión de sus predecesores. Al habilitar la adquisición automática de espectros parciales de absorción, facilita la realización de experimentos que permiten estudiar la estructura de los materiales en forma dinámica.

La aplicación de nuevas tecnologías al diseño del instrumental resultó en la implementación de un espectrómetro más versátil que sus antecesores, que consigue ampliar el espectro de aplicación al agregar nueva funcionalidad a los instrumentos. Resulta así un universo de nuevos experimentos realizables que permiten la medición de nuevos parámetros físicos, como así también una gran cantidad de experimentos de larga duración que pueden ser automatizados.

La estructura de la tesis se describe brevemente a continuación.

En el Capítulo 1 se introducen los fundamentos del efecto Mössbauer y de la técnica de medición. El propósito es definir las variables de interés y determinar los factores que las afectan. En el Capítulo 2 se presentan los principales aspectos relacionados con la instrumentación, indicando algunos puntos sensibles de mejora. Sobre el final del capítulo se realiza la propuesta del nuevo espectrómetro de velocidad programable, planteando claramente los objetivos de la tesis.

Los Capítulos 3 a 5 constituyen la sección de materiales y métodos. Se organizó en tres partes para dedicar un capítulo a los aportes realizados para cada uno de los bloques principales en 
que se dividió la instrumentación del experimento. Estos bloques se nombraron etapa de adquisición, de accionamiento y de procesamiento.

En el Capítulo 6 se presentan y discuten los resultados experimentales obtenidos. Primero se muestra un procedimiento de calibración que permite caracterizar el desempeño del nuevo instrumento, y luego la implementación de algunos experimentos novedosos. Finalmente se exponen las conclusiones y se enumeran algunas líneas de trabajo interesantes para el futuro.

Los apéndices agrupan diseños o líneas de trabajo afines al tema, que se desarrollaron en simultáneo con la tesis, pero que no integran el eje principal de trabajo. 


\section{Marco de trabajo}

Esta tesis se llevó a cabo en dos laboratorios experimentales de la Universidad Nacional de La Plata (UNLP) que registran más de 30 años de actividades en conjunto. Se mencionarán brevemente los antecedentes de cada uno de ellos.

El Laboratorio de Espectroscopia Nuclear e Interacciones Hiperfinas (LENIH) del Departamento de Física, Facultad de Cs. Exactas de la UNLP, perteneciente al Instituto de Física (IFLP) del CONICET, comenzó su actividad en la década del 70. Un número importante de investigadores, estudiantes y técnicos se dedican al estudio de los problemas físicos relacionados con la materia condensada. Entre sus integrantes cuenta con especialistas en Espectroscopia Mössbauer y Correlaciones Angulares Perturbadas. Actualmente, seis lineas de investigación contribuyen al conocimiento de la estructura nanoscópica de los materiales y su relación con las propiedades macroscópicas de los mismos.

El Laboratorio de Electrónica del Departamento de Física trabaja en forma coordinada con el Laboratorio de Electrónica Industrial, Control e Instrumentación (LEICI) de la Facultad de Ingeniería, a partir de un Convenio de Colaboración Mutua celebrado en febrero de 1978 entre las Facultades de Ciencias Exactas e Ingeniería de la UNLP. El intercambio de instrumental, la realización de seminarios comunes y la programación conjunta de actividades, han dado lugar a una complementación tanto en los aspectos temáticos como en los de financiamiento. El Laboratorio atiende los requerimientos de los laboratorios del Departamento de Física en lo que se refiere al desarrollo de equipamiento auxiliar para aplicaciones específicas, requerimientos de nuevas configuraciones experimentales y apoyo para la puesta en marcha de nuevos experimentos.

En el período 1997-1999, siendo miembro del Laboratorio de Electrónica, presenté mi Tesis de Magister de la UNLP en el tema Sistemas jerárquicos de tiempo real para la adquisición de datos y control. Las técnicas desarrolladas encontraron aplicación en la implementación de instrumental para experimentos Mössbauer, lo cual abrió la posibilidad de nuevas aplicaciones y trabajo en conjunto. La actividad se retomó en el año 2003 hasta la actualidad, dando como fruto esta Tesis.

Deseo expresar mi sincero agradecimiento a los integrantes del Laboratorio de Barridos Térmicos Mössbauer del LENIH: Francisco Sánchez, Gustavo Pasquevich, Pedro Mendoza Zélis y Marcela Fernández Van Raap, por su confianza, soporte permanente y largas tardes de interesantes discusiones. Esta tesis no hubiera sido posible sin el interés y dedicación que ellos mostraron desde que las primeras ideas empezaron a ser evaluadas, hace ya varios años. En especial a Gustavo Pasquevich, cuya tesis para obtener el grado de Doctor en Física fue desarrollada en forma simultánea con ésta, produciendo una realimentación realmente fructífera y estimulante.

En los últimos años se produjeron en conjunto múltiples publicaciones internacionales, como así también gran cantidad de presentaciones a congresos, tanto nacionales como 
internacionales. Los detalles de dichas publicaciones se presentan en un apéndice de esta tesis.

Los instrumentos desarrollados se encuentran actualmente operando en laboratorios del LENIH y produciendo datos para un número importante de investigaciones en curso. 


\section{Contenido}

\section{Capítulo 1}

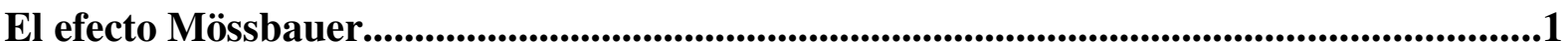

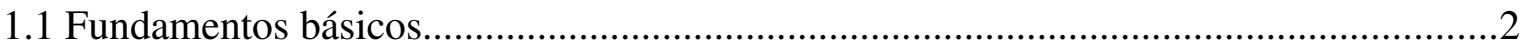

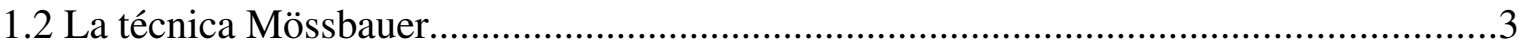

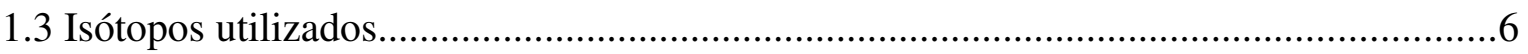

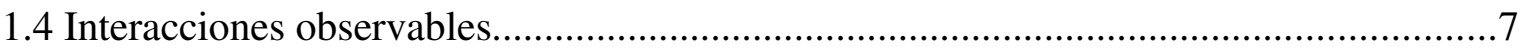

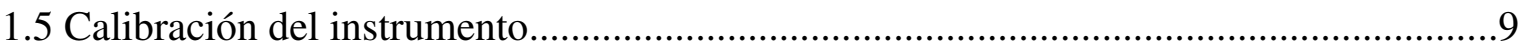

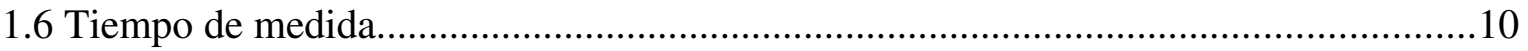

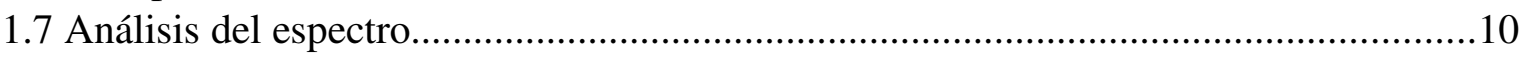

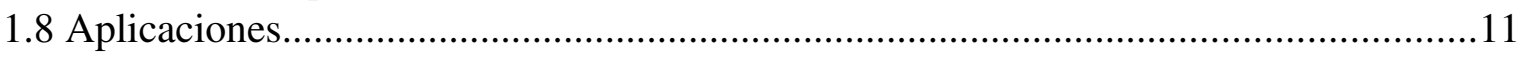

\section{Capítulo 2}

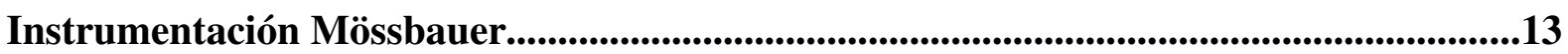

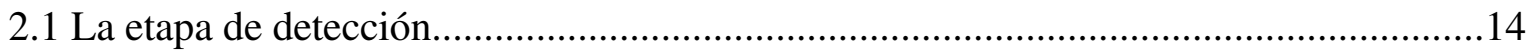

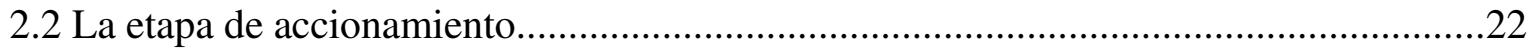

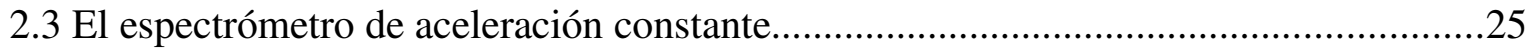

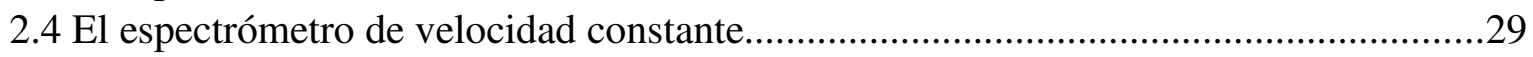

2.5 Propuesta para un nuevo espectrómetro de velocidad programable..............................35

\section{Capítulo 3}

Optimización de la etapa de detección.........................................................................41

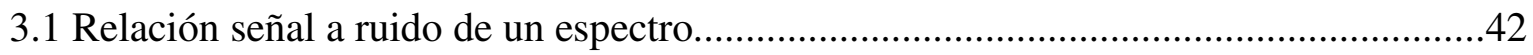

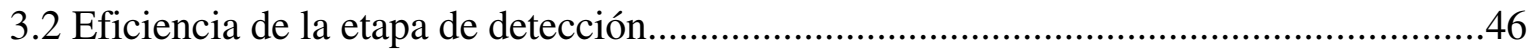

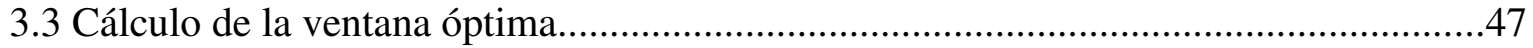

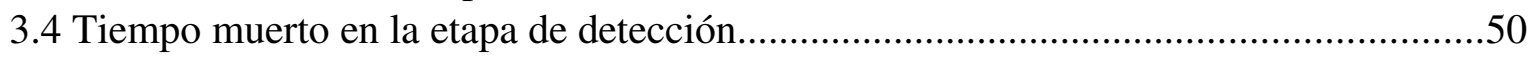

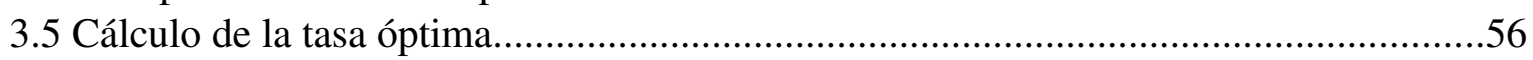

$3.6 \mathrm{El}$ procedimiento de calibración propuesto............................................................6

\section{Capítulo 4}

La etapa de accionamiento a velocidad programable.................................................63

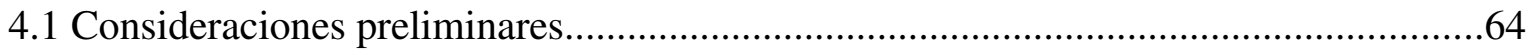

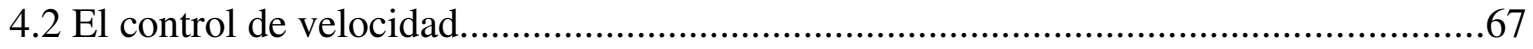

4.3 Técnica de accionamiento con realimentación de posición..........................................75

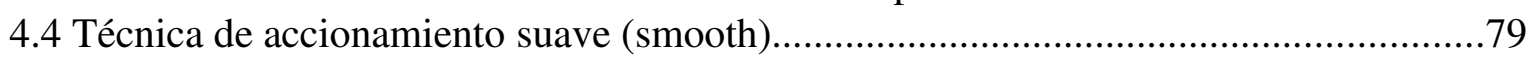

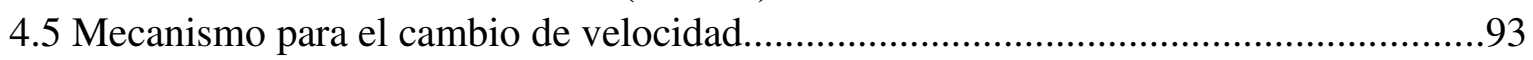

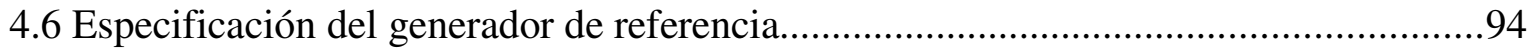

\section{Capítulo 5}

La etapa de procesamiento programable........................................................................97

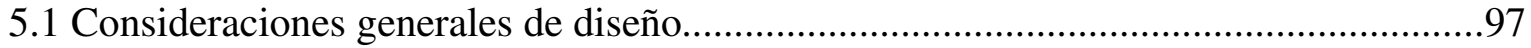

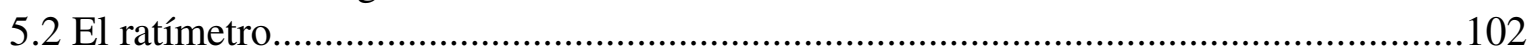

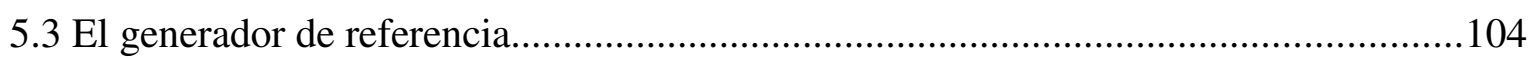

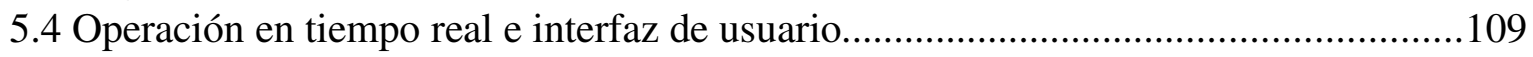




\section{Capítulo 6}

Aplicaciones y resultados.

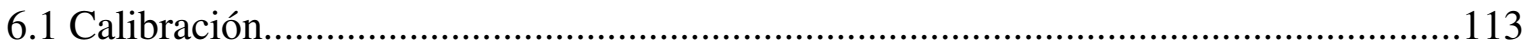

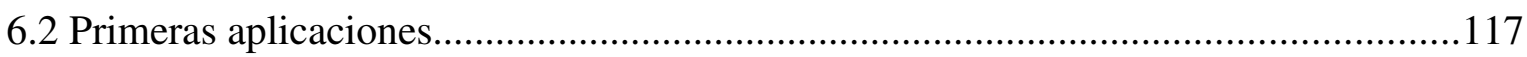

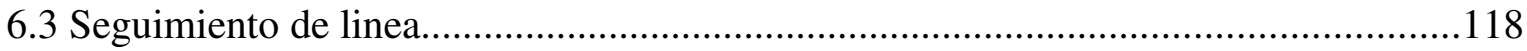

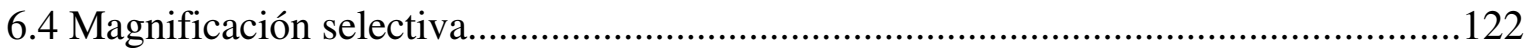

\section{Capítulo 7}

Conclusiones, aportes originales y líneas abiertas de trabajo.

Líneas abiertas de investigación.

Apéndice A

Mejoras en la etapa de detección.

Apéndice B

Barridos de campo magnético.

Apéndice $\mathbf{C}$

Control de velocidad con realimentación de posición.

Apéndice D

Publicaciones relacionadas con la tesis.

Bibliografía.

Referencias. 


\section{Capítulo 1 El efecto Mössbauer}

El efecto Mössbauer, también conocido como absorción resonante de rayos gamma, es un fenómeno descubierto por el físico alemán Rudolf Ludwig Mössbauer a fines de la década del 50 [Mössbauer 1958], lo cual le valió la obtención del Premio Nobel de Física en 1961. Desde entonces, la técnica experimental que explota las propiedades de este fenómeno se ha transformado en una herramienta muy utilizada para el estudio de la estructura atómica y nuclear de la materia, como así también de sus propiedades químicas y magnéticas.

Al igual que otras técnicas de observación nuclear (resonancia magnética, correlaciones angulares perturbadas, reacciones nucleares resonantes, etc.) el efecto Mössbauer encuentra diversas aplicaciones en física y química de la materia condensada. Tal es el caso de la física de sólidos, magnetismo, física metalúrgica, mineralogía, biología y arqueología de las Bellas Artes [De Benedetti 1960].

Se trata de una técnica de resonancia nuclear que utiliza la emisión libre de retroceso de rayos gamma desde un núcleo radiactivo, seguido por la absorción selectiva de dichos fotones en otro núcleo cercano. El fenómeno es extremadamente sensible a pequeñas diferencias entre la estructura del núcleo emisor y el núcleo absorbente. La absorción depende fuertemente del estado electrónico, químico y magnético de los átomos que componen el material en estudio [Cranshaw 1974].

A lo largo de este capítulo se presentarán los aspectos más relevantes de esta técnica, desde el punto de vista de la instrumentación utilizada en los laboratorios. Un análisis detallado de la física asociada al efecto Mössbauer puede encontrarse en la bibliografía específica recopilada al final de la tesis. 


\subsection{Fundamentos básicos}

Para entender el mecanismo del efecto Mössbauer es necesario comprender previamente algunos principios fundamentales como la absorción resonante, la emisión libre de retroceso y el efecto Doppler, los cuales se detallan a continuación.

\subsubsection{Absorción resonante}

Al igual que la nube de electrones del átomo, el núcleo puede existir sólo en ciertos estados de energía definidos. Una fuente radiactiva emite un fotón durante la transición desde un nivel determinado de energía a uno de energía inferior. Estos fotones de alta energía (desde $\mathrm{KeV}$ hasta unos pocos $\mathrm{MeV}$ ) se denominan rayos gamma. En el proceso inverso, para que un fotón pueda ser absorbido por un núcleo, su energía debe ser exactamente igual a la diferencia entre dos de dichos estados. Este fenómeno se denomina absorción resonante.

El factor más importante en la distribución de energía de los fotones emitidos es la vida media del estado excitado $\tau$. El principio de incertidumbre de Heinsenberg relaciona las incertidumbres de tiempo $\Delta t$ y energía $\Delta E$ (dos variables canónicas conjugadas) con la constante de Plank $\left(h=6,626 \times 10^{-34} J s\right)$, tal que $\Delta E . \Delta t \geq h / 2 \pi$. El nivel de referencia tiene vida infinita y por lo tanto no tiene incertidumbre en su energía. En cambio, un estado excitado tiene una vida media de un microsegundo o menos, por lo tanto existirá una distribución en la energía de los rayos gamma con un ancho $\Gamma$ a mitad del máximo, tal que:

$$
\Gamma \tau=\frac{h}{2 \pi}
$$

La distribución de energía de los fotones emitidos y absorbidos $N(E)$ es una lorentziana de parámetro $\Gamma$ dada por:

$$
\mathrm{N}(E)=\frac{1}{4} \frac{\Gamma^{2}}{(E-E .)^{2}+\left(\frac{\Gamma}{4}\right)^{2}}
$$

donde $E_{0}$ es la energía del estado excitado menos la energía del estado fundamental [Greenwood and Gibb 1971, Chapter I].

\subsubsection{Emisión libre de retroceso}

Un rayo gamma que es emitido por un núcleo perteneciente a un átomo libre no puede ser absorbido por un núcleo similar de otro átomo ya que su energía es menor que la de resonancia. Esto se debe al retroceso que le imprime la emisión al núcleo que está libre. Si en cambio el núcleo emisor se encuentra formando parte de la red de un sólido, la masa del sistema se incrementa drásticamente y la energía consumida en el retroceso es despreciable.

Este concepto puede ser ilustrado por el comportamiento de un rifle. Si el rifle se sostiene débilmente durante un disparo, su retroceso (o culatazo) será violento. Si se lo sostiene 
firmemente contra el hombro, el retroceso será reducido considerablemente. El momento del sistema que dispara debe ser igual y opuesto al del proyectil (conservación del momento). Sosteniendo el rifle firmemente el tirador incluye la masa de su cuerpo al sistema de disparo, reduciendo la velocidad de retroceso.

\subsubsection{Efecto Doppler}

Cuando el núcleo emisor está en movimiento respecto del absorbente, los fotones emitidos experimentan un corrimiento de energía. La energía de un fotón emitido por una fuente que se mueve a velocidad mucho menor que la de la luz puede expresarse como:

$$
E_{\gamma}=E_{0}\left(1+\frac{v}{c}\right)
$$

$E_{0}$ es la energía del fotón emitido por un núcleo en reposo, $v$ es la velocidad de la fuente respecto del absorbente y $c$ la velocidad de la luz. El incremento de energía del fotón es proporcional a la velocidad relativa de la fuente. Variando dicha velocidad es posible variar la energía de los fotones con gran resolución dentro de un rango muy pequeño (del orden del ancho de los niveles atómicos).

\subsection{La técnica Mössbauer}

Rudolph Mössbauer descubrió que, para ciertos niveles de energía, un fotón puede ser emitido desde un núcleo perteneciente a una red sólida, la cual retrocede como una única masa rígida. En estas condiciones la energía perdida en el retroceso es despreciable y el fotón emitido puede excitar a un núcleo absorbente idéntico que se encuentra en una red próxima [Mössbauer 1958]. En estas circunstancias, demostró que una fracción $f_{L M}$ de los rayos gamma es emitida libre de retroceso, pudiendo ser absorbida por otro núcleo idéntico. La fracción $f_{L M}$, llamada fracción Lamb-Mössbauer, está dada por:

$$
f_{L M}=e^{-K^{2}\left[x^{2}\right]}
$$

donde $K=2 \pi / \lambda, \lambda$ es la longitud de onda del rayo gamma y $\left[x^{2}\right]$ es el valor cuadrático medio del desplazamiento térmico del átomo.

Este factor disminuye cuando la energía de los fotones aumenta (disminuye su longitud de onda) o aumenta la temperatura. En el caso muy favorable del $\mathrm{Fe}^{57}$ puede alcanzar un $70 \% \mathrm{o}$ más a temperatura ambiente. En casos menos favorables, como es el caso del $\mathrm{W}^{182}, f_{L M}$ es menor que el $1 \%$ a temperatura de He [Cranshaw 1974].

La técnica Mössbauer consiste en utilizar el mismo tipo de núcleo en dos sólidos. En uno de ellos se utiliza el núcleo en estado de radiación o excitado y en el otro en estado absorbente o fundamental. Así es posible absorber en el segundo la radiación gamma del primero. El método es tan sensible que si un sólido se mueve con relación al otro con una velocidad tan baja como un milímetro por segundo la resonancia es destruida por el efecto Doppler. La absorción resonante también desaparecerá si los estados de energía, tanto de la fuente como del absorbente, son afectados por algún parámetro externo, como por ejemplo ante la 


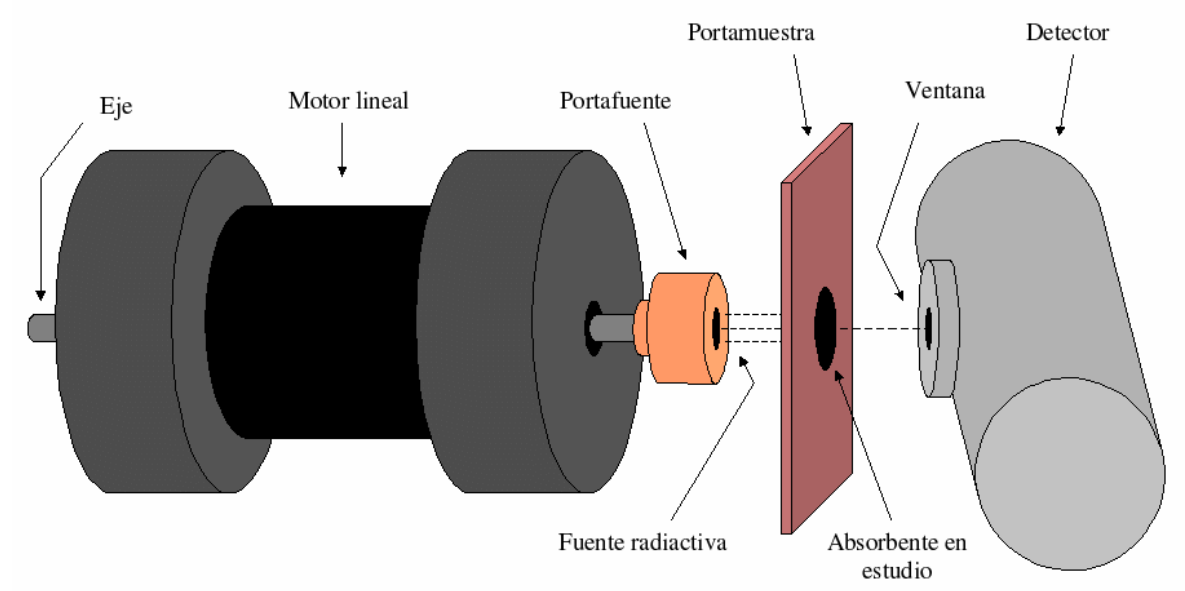

Figura 1: Disposición del transductor electromecánico, la fuente, el absorbente en estudio y el detector en un espectrómetro Mössbauer típico.

aplicación de campo magnético o la variación de la temperatura. En este caso puede variarse la velocidad relativa entre la fuente y el absorbente hasta restablecer la absorción resonante, pudiéndose así cuantificar los efectos de dicho parámetro.

A los fines de la implementación, la fuente radiactiva se monta sobre un transductor electromecánico (usualmente un motor lineal) que le imprime un movimiento longitudinal oscilatorio de valor medio nulo. Los fotones emitidos por dicha fuente sufren una modulación de su energía debido al efecto Doppler, desde un mínimo a la máxima velocidad negativa, pasando por velocidad nula hasta un máximo a la máxima velocidad positiva. Con el eje de dicho transductor se alinean el absorbente que se desea estudiar y un detector de rayos gamma, como muestra esquemáticamente la Figura 1.

Cada fotón que llega al absorbente tiene una cierta probabilidad de resonar en un núcleo idéntico. El resto de los fotones atraviesan el material sin ser absorbidos. Los núcleos que atrapan fotones son llevados a un nivel excitado de energía. Luego de un tiempo en ese estado (vida media del estado excitado) un nuevo fotón será emitido isotrópicamente (en cualquier dirección), con lo cual la probabilidad de que llegue al detector puede ser considerada despreciable. Por lo tanto, los fotones que tienen la cantidad de energía exacta como para quedar atrapados en un núcleo del absorbente nunca alcanzan al detector.

Al representar la transmisión del material (tasa de conteo del detector) en función de la velocidad de la fuente se obtiene un espectro de absorción como el de la Figura 2, donde la disminución de la tasa de conteo es causada por la absorción resonante. En el caso de la figura, la máxima absorción se encuentra en el centro del espectro (velocidad nula), lo cual implica que los estados de energía del absorbente son idénticos a los de la fuente. En la Sección 1.4 se muestran las singularidades más frecuentes que pueden experimentar dichos niveles y que pueden ser observadas por medio de esta técnica. La forma de la línea de absorción se debe a la convolución de la distribución lorentziana de la energía del nivel emisor con la del absorbente (ambas de ancho $\Gamma$ ), resultando en un ancho teórico de $2 \Gamma$.

En una implementación de un espectrómetro Mössbauer pueden identificarse tres bloques fundamentales relativos a la instrumentación: 

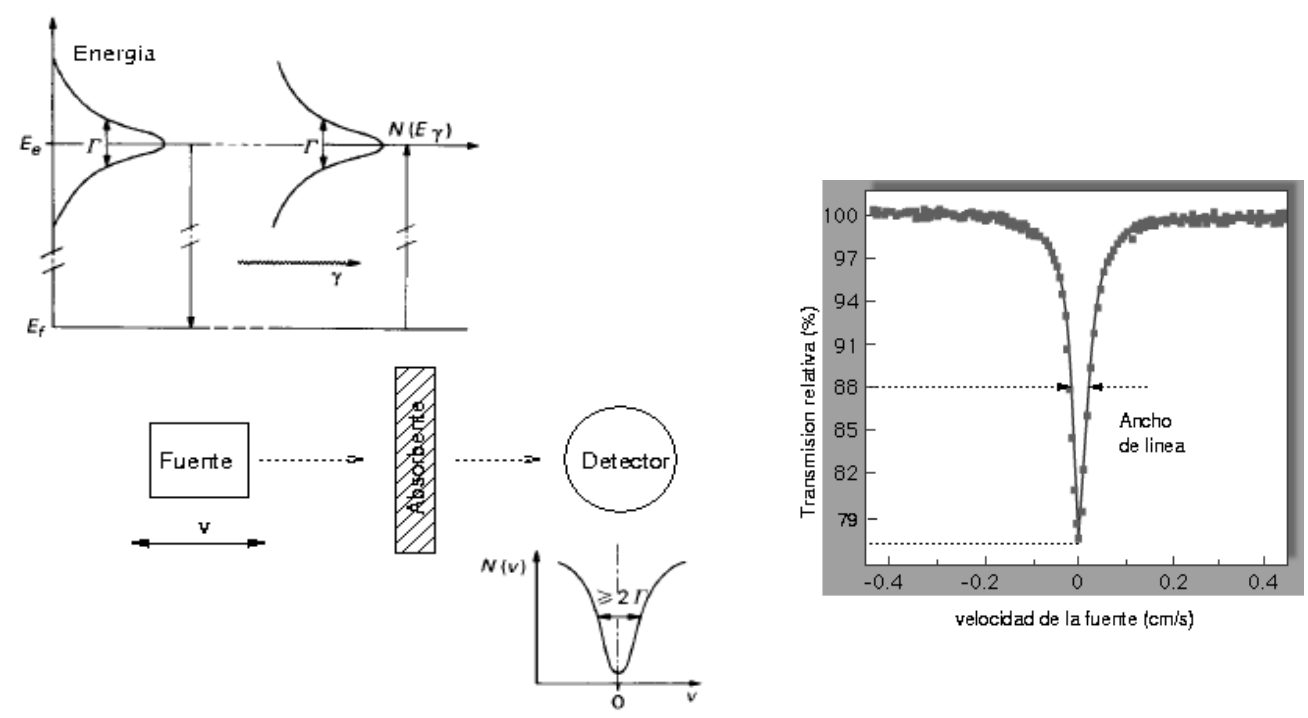

Figura 2: a) Diagrama esquemático de los niveles energéticos de la fuente y el absorbente, mostrando el efecto resultante en el detector. b) Espectro Mössbauer experimental dado por la transmisión relativa de rayos gamma del absorbente en función de la velocidad de la fuente.

(i) Etapa de detección. Compuesta por el detector, preamplificador, amplificador y discriminador. Este conjunto es el encargado de convertir en pulsos lógicos los fotones detectados que poseen la energía de interés, para que luego puedan ser contados por la etapa de procesamiento. La instrumentación involucrada en esta etapa se presenta en detalle en la Sección 2.1.

(ii) Etapa de accionamiento. Integrada por un transductor electromecánico $\mathrm{y}$ su correspondiente controlador de velocidad. Este conjunto permite modificar la energía de los fotones emitidos por la fuente, accionando sobre la velocidad de desplazamiento de la misma. Un análisis de las opciones y los detalles constructivos de este bloque puede encontrarse en la Sección 2.2.

(iii) Etapa de procesamiento. Este conjunto de instrumentos es el encargado de computar la transmisión del absorbente (conteo de fotones) en función de la energía (velocidad de la fuente). Para ello debe ser capaz de seleccionar la velocidad en un cierto rango (suministrando la correspondiente referencia de velocidad a la etapa de accionamiento) y calcular la transmisión del material para dicha energía (contando la tasa de eventos proveniente de la etapa de detección). La información generada tiene la forma del espectro de la Figura 2. En la Sección 2.3 se presentan las diferentes opciones que existen para la implementación de este bloque, cada una con sus características particulares. Sólo digamos por ahora que esta etapa es capaz de entregar el conteo en función de la velocidad, para una velocidad fija o para un determinado rango de velocidades.

Para la implementación de la técnica descripta, usualmente llamada de transmisión, se utilizan fuentes radiactivas relativamente intensas (20 a 100 milicurie). Solo puede ser aplicada a muestras lo suficientemente delgadas para que puedan ser atravesadas por los fotones. La preparación de la muestra es un punto crítico de este tipo de experimentos que está tratado en detalle en la bibliografía especializada [Greenwood and Gibb 1971, Cap. 2.6 
Source and Absorber Preparation]. En este punto debe tenerse en cuenta que si la muestra es muy delgada, disminuye la probabilidad de que la absorción resonante se produzca, disminuyendo el efecto. En cambio, si la muestra es muy gruesa, si bien aumenta la probabilidad de absorción, también aumenta la probabilidad de que los fotones de cualquier energía colisionen con la red y no lleguen al detector, disminuyendo así la eficiencia del experimento debido a la baja estadística. Existe un compromiso entre ambas variables, pudiéndose calcular el espesor óptimo. Debe considerarse además que existe un límite hasta el cual es aceptable la aproximación de las líneas de absorción con una lorentziana, llamado generalmente límite de espesor delgado. Más allá de este límite las líneas se deforman, aproximándose a gaussianas. En general, el espesor óptimo suele estar más allá del límite de espesor delgado. Esto implica que si se desea obtener un buen espectro en poco tiempo (espesor óptimo) debe aceptarse una ligera deformación de las líneas. Esta deformación puede ser tenida en cuenta en el momento del ajuste del espectro.

Existe una variante de la técnica de transmisión descripta, menos utilizada por ser menos efectiva, llamada de reflexión (scattering), en la cual pueden utilizarse muestras voluminosas sin preparación. El haz incidente de rayos gamma se impacta contra la superficie del material en estudio, produciendo emisión de electrones de conversión o rayos X. Dentro de las principales características de este tipo de experimentos puede mencionarse que es no destructivo y que trabaja con muestras sólidas. La principal diferencia con el método de transmisión es que por este medio sólo se obtiene información respecto de la superficie del material en estudio. En lo que sigue, al mencionar la técnica Mössbauer se estará haciendo referencia implícitamente a la técnica Mössbauer de transmisión.

En general, tanto en transmisión como en reflexión, la técnica Mössbauer tiene una relativa simplicidad experimental. La instrumentación es adaptable para mediciones portátiles [Klingelhöfer 2002] [De Souza 2003], como así también en altas y bajas temperaturas. Una propiedad interesante de esta técnica es que cuando el material consta de varias fases el espectro resultante es la suma de los espectros individuales. En el caso particular de muestras delgadas la intensidad del espectro de cada componente es proporcional a su concentración [Cranshaw 1974]. Este método permite también poner de manifiesto los cambios que se presentan en los niveles de energía debido a la variación de la temperatura de la muestra [Chien 1977] o del campo magnético al cual está sometida la misma.

Pero la principal característica del efecto Mössbauer es que se evidencia muy notoriamente en el hierro y sus compuestos. Por este motivo se ha transformado en una herramienta de estudio muy importante para este tipo de materiales, predominantes en el desarrollo tecnológico.

\subsection{Isótopos utilizados}

El efecto Mössbauer ha sido observado en más de 100 isótopos, de los cuales el primero fue $\operatorname{Ir}^{191}$, utilizado por Rudolf Mössbauer en 1957. Son condiciones fundamentales para su aplicación que el isótopo posea un estado fundamental estable y un estado excitado de baja energía que decaiga en emisión gamma. El estado excitado debe ser de baja energía para que la emisión esté libre de retroceso. Esto ocurre sólo si la energía de retroceso es pequeña comparada con la energía de vibración de la red, lo que generalmente restringe el efecto 


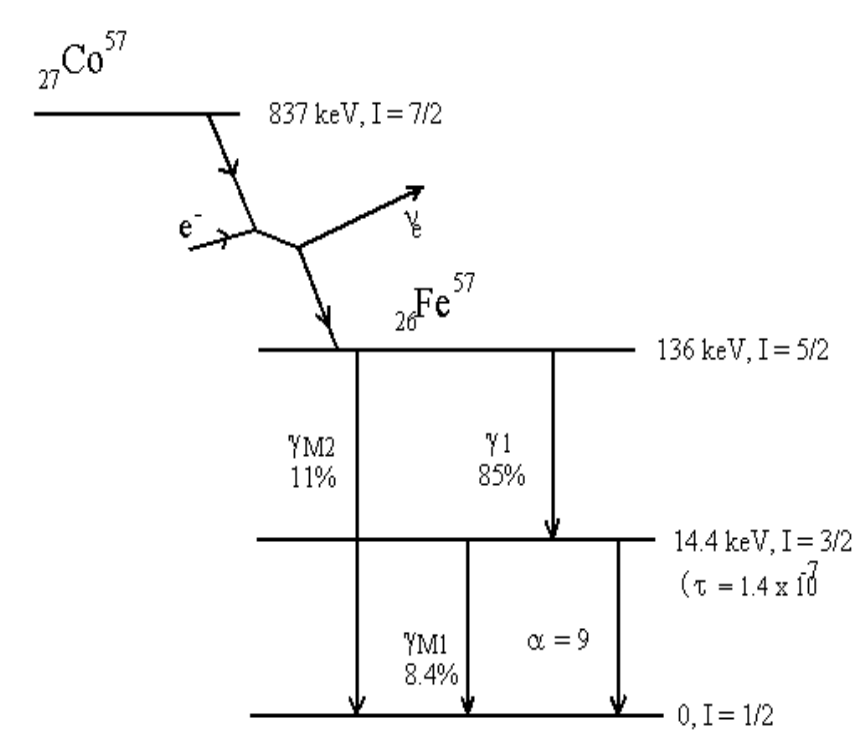

Figura 3: Niveles de energía del Fe $\mathrm{F}^{57}$. La espectroscopia Mössbauer involucra la transición de $14.4 \mathrm{KeV}$. Las intensidades están dadas en \% de decaimientos.

Mössbauer a rayos gamma con energías menores a $150 \mathrm{KeV}$

El isótopo más utilizado, dadas sus buenas características y costo, es el $\mathrm{Co}^{57}$ que decae en $\mathrm{Fe}^{57}$ con estados del núcleo excitados. En la Figura 3 se muestran los niveles de energía de este isótopo. Cerca del $10 \%$ de todos los núcleos de $\mathrm{Fe}^{57}$ excitados emitirán un rayo gamma de 14.4 KeV durante una transición desde el estado metaestable $\mathrm{I}=3 / 2$ al estado fundamental $\mathrm{I}=$ $1 / 2$ (siendo I el spin nuclear). La cantidad de fotones de $14.4 \mathrm{KeV}$ libres de retroceso, comparado con todos los fotones de $14.4 \mathrm{KeV}$ emitidos, varía con las propiedades del sólido y su temperatura (ver factor $f_{L M}$ en la sección anterior). El ancho de línea de la radiación emitida está determinado por la vida media del estado $\mathrm{I}=3 / 2$.

En el $\mathrm{Fe}^{57}$ la vida media $\tau$ de este estado es de $1.4 \times 10^{-7}$ segundos y la distribución de energía

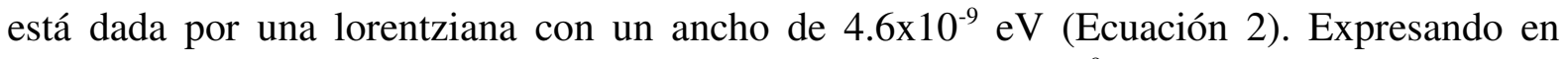
velocidad (Ecuación 3) y siendo $\mathrm{E}_{0}=14.4 \mathrm{KeV}, \Delta \mathrm{E}=4.6 \times 10^{-9} \mathrm{eV} \mathrm{y} \mathrm{c}=299792458 \mathrm{~m} / \mathrm{s}$, resulta $2 \Gamma=0.192 \mathrm{~mm} / \mathrm{s}$, al cual llamaremos valor teórico del ancho de línea para el hierro.

Otro isótopo bastante utilizado, aunque en menor medida, es el $\mathrm{Sn}^{119}$, en el cual también puede observarse claramente el efecto Mössbauer. En algunos materiales el efecto se hace visible solo a muy bajas temperaturas.

\subsection{Interacciones observables}

Las energías de los niveles nucleares dependen de múltiples interacciones entre las que cabe mencionar las interacciones entre el núcleo y los electrones que lo rodean (interacciones hiperfinas). En el espectro pueden observarse corrimientos o desdoblamientos de los niveles de energía del núcleo, dependiendo del tipo de interacción, como muestra la Figura 4. La observación de estos fenómenos es posible debido a que el ancho de los niveles atómicos (del orden de $\left.10^{-8} \mathrm{eV}\right)$ es menor que la magnitud de dichas interacciones $\left(10^{-7} \mathrm{a} 10^{-8} \mathrm{eV}\right)$. De la 


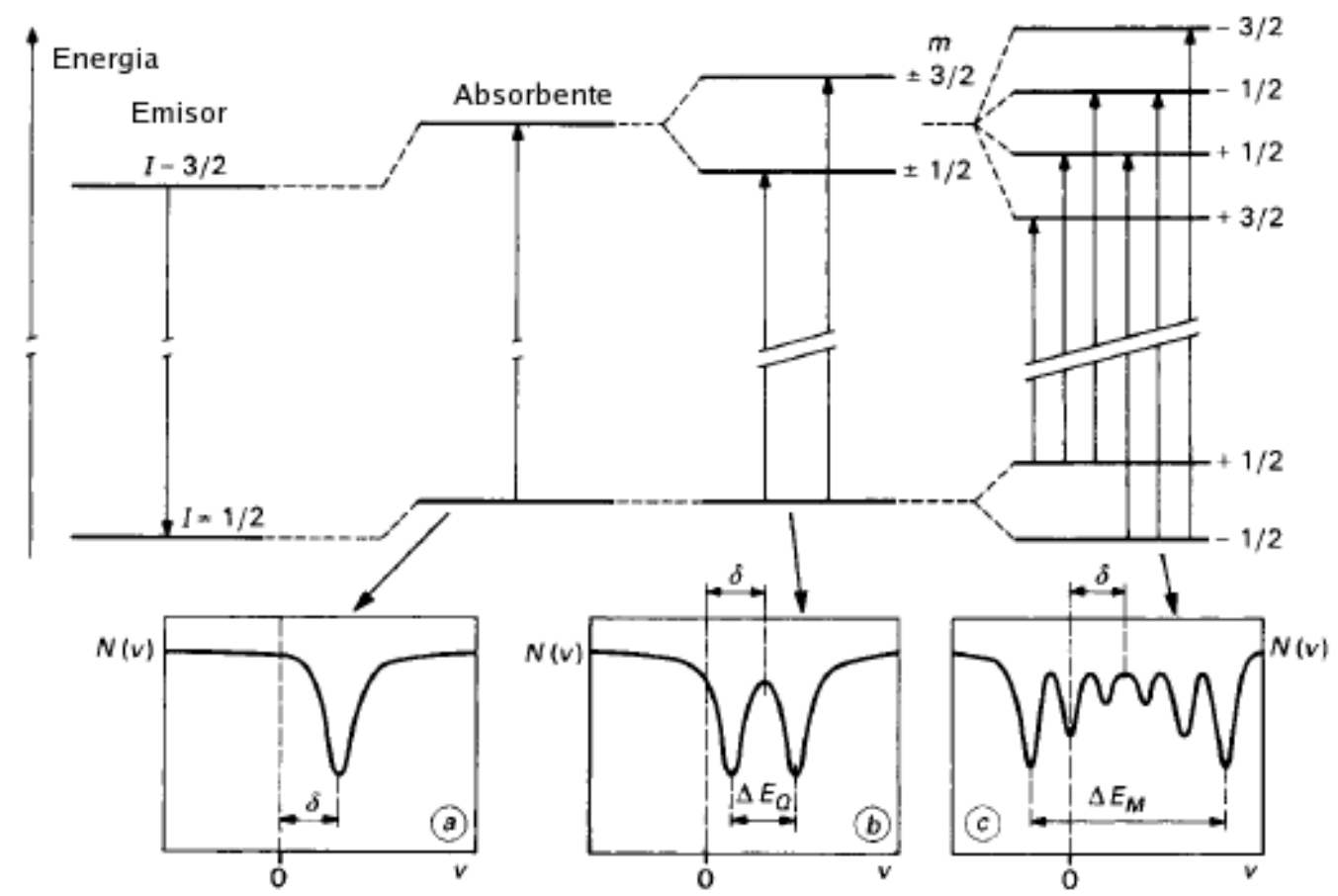

Figura 4: Interacciones observables: a) corrimiento isomérico, b) desdoblamiento cuadrupolar y c) desdoblamiento magnético [Eymery et al 2000].

Ecuación 3 puede deducirse que para detectar interacciones de este orden son necesarias velocidades de algunos $\mathrm{mm} / \mathrm{s}$, lo cual es experimentalmente factible.

A continuación se detallan las singularidades más frecuentes.

\subsubsection{Interacción monopolar eléctrica}

En un determinado material puede existir una diferencia de energía entre los niveles excitado y fundamental de la fuente y del absorbente, debido a la diferencia de densidades electrónicas en el volumen finito del núcleo atómico en la fuente y el absorbente. La contribución que da lugar al corrimiento isomérico es la energía de interacción entre la carga del núcleo y el potencial debido a los electrones de su entorno. El espectro resultante en este caso presenta un corrimiento del mínimo de transmisión respecto del cero de velocidad denominado corrimiento isomérico $(\delta)$.

\subsubsection{Interacción cuadrupolar eléctrica}

Esta consiste en la interacción entre el momento cuadrupolar eléctrico del núcleo y el gradiente de campo eléctrico debido a los electrones (que depende de la distribución de carga electrónica). Esta distribución está dominada por la estructura, las ligaduras atómicas y la consecuente distribución de los orbitales (por ejemplo, la estructura hexagonal de $\mathrm{Fe}(\mathrm{OH})_{2}$ presenta a $90 \mathrm{~K}$ un doblete de $3 \mathrm{~mm} / \mathrm{s})$. El parámetro observable se denomina separación cuadrupolar $\left(\Delta E_{Q}\right)$. 


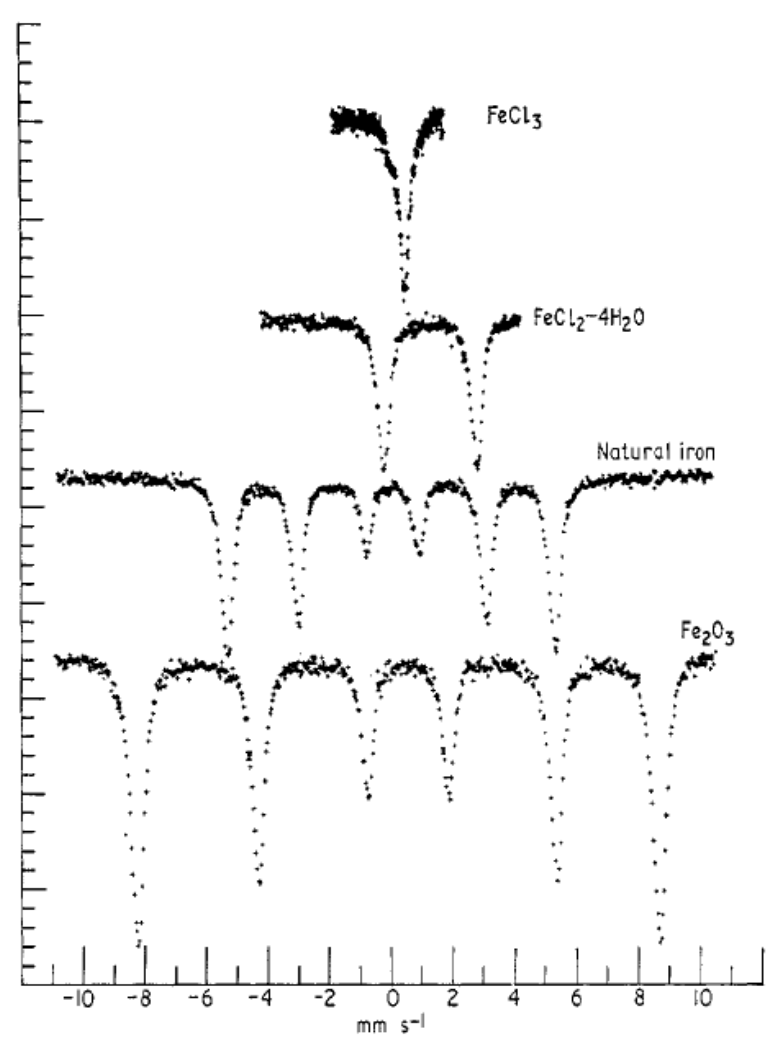

Figura 5: Espectros experimentales de algunos compuestos [Cranshaw 1974]

\subsubsection{Interacción dipolar magnética}

Se debe a la interacción entre el momento dipolar magnético del núcleo y el campo magnético en la posición del núcleo. Este campo magnético puede deberse a una fuente externa o interna (ferromagnetismo, ferrimagnetismo o antiferromagnetismo). En este caso se desdoblan tanto el estado fundamental como el excitado, resultando en general un espectro con seis mínimos de transmisión (sextete). Esta es la forma del conocido espectro de una lámina de hierro natural que se utiliza como calibración de los espectrómetros. El parámetro observable es el campo magnético efectivo $\left(B \approx \Delta E_{M}\right)$.

Estos tres parámetros (corrimiento isomérico, separación cuadrupolar y campo magnético efectivo) son la principal información extraída de un espectro Mössbauer, aunque pueden presentarse situaciones más complicadas que requieran un tratamiento más detallado. En la Figura 5 pueden verse los espectros de algunos materiales.

\subsection{Calibración del instrumento}

El espectrómetro se calibra usualmente utilizando un absorbente de referencia con un espectro bien conocido, como por ejemplo una lámina de hierro delgada. El cero de velocidad se toma en el centro del espectro de referencia a temperatura ambiente. Los anchos de línea observados durante el transcurso de una calibración de este tipo son mayores que el valor 
teórico de $0.19 \mathrm{~mm} / \mathrm{s}$ en el caso del $\mathrm{Fe}^{57}$. Esto se debe al espesor de la muestra, a imperfecciones del control de velocidad y a la presencia de defectos estructurales en el absorbente. Es esperable obtener anchos de línea de $0.21 \mathrm{~mm} / \mathrm{s}$, lo cual se considera aceptable. El parámetro ancho de línea se utiliza como una medida de la calidad del espectro y es medido (en $\mathrm{mm} / \mathrm{s}$ ) como el ancho de la línea de transmisión al $50 \%$ de la atenuación máxima. Para calibrar la velocidad con mayor precisión pueden utilizarse técnicas de interferometría con láser [Waard 1965] [Cranshaw 1973] [Player 1976] [Yoshimura 1977] [Filter 1996].

\subsection{Tiempo de medida}

La emisión de fotones gamma puede modelarse como un proceso aleatorio con una cierta tasa media y una varianza. A mayor cantidad de eventos registrados, menor será el error estadístico, mayor la resolución de la medida y también mayor el tiempo necesario para realizarla.

Es posible disminuir el tiempo de medida aumentando la tasa de eventos registrados. Dicha tasa es inversamente proporcional al cuadrado de la distancia entre la fuente y el detector, por lo que acercando la fuente al detector se produce un aumento de la tasa. Pero esta distancia no puede ser acortada en exceso por dos motivos: distorsiones en el espectro debido a los tiempos muertos de la etapa de detección y al efecto de apilamiento [Müller 1973] (ver Sección 2.1), y además distorsiones debidas al ángulo sólido de emisión de la fuente, por lo que el recorrido entre extremos de la fuente debe ser despreciable respecto de la distancia entre la fuente y el detector. La tasa de conteo estará afectada también por el espesor de la muestra (ver Sección 1.2), por el nivel de actividad de la fuente radiactiva y por el criterio de selección de los eventos.

Todos estos factores llevan a que exista un compromiso entre la calidad del espectro obtenido y el tiempo de medida. En la práctica resulta que para obtener una resolución aceptable son necesarias varias horas de medición. En el Capítulo 3 se presenta un estudio más detallado de estos aspectos y se propone un criterio de optimización.

\subsection{Análisis del espectro}

El espectro resultante estará formado por una cierta cantidad de puntos, correspondientes a diferentes velocidades o energías. Este espectro es procesado posteriormente para obtener los parámetros buscados. El método más usual de extracción de la información es ajustando el resultado experimental a un espectro teórico apropiado para la situación en estudio [Azevedo 1997]. El espectro teórico está compuesto por una superposición de lorentzianas del doble de ancho que el ancho de línea natural del material, cuyas posiciones y profundidades deben ser ajustadas hasta minimizar el error.

Existen algoritmos de ajuste más sofisticados que incluyen compensación por efecto del ángulo sólido, ensanchamiento de la línea debido al espesor de la muestra, y otros parámetros que apartan los datos experimentales de las predicciones teóricas. Este procesamiento se realiza generalmente fuera de línea, por lo cual el equipamiento de laboratorio no necesita disponer de una gran potencia de cómputo. 


\subsection{Aplicaciones}

El efecto Mössbauer se aplica en varias áreas de la ciencia. Además de permitir la medición de la interacción del núcleo con el entorno, esta técnica provee una fuente de radiación electromagnética (rayos gamma) cuya frecuencia está definida con mucha precisión. Utilizando rayos gama libres de retroceso pueden obtenerse resoluciones mejores que $10^{-12}$. En estudios de la relatividad, aprovechando la gran precisión con que los rayos gamma pueden medirse, puede obtenerse una demostración directa de la atracción gravitatoria sobre la radiación electromagnética.

Las aplicaciones en física nuclear son numerosas. El efecto Mössbauer permite medir directamente el ancho de línea de la resonancia de rayos gamma, el cual se corresponde con el ancho de línea del nivel emisor; el tiempo de vida del estado excitado puede deducirse de este ancho. El momento del cuadrupolo eléctrico y del dipolo magnético del núcleo pueden ser medidos por el desdoblamiento de los niveles nucleares en componentes hiperfinas, detectables en un espectro Mössbauer, como se mencionó en la Sección 1.4.

En física de estado sólido se aplica en dinámica de redes e interacciones hiperfinas. La probabilidad de que el proceso de emisión esté libre de retroceso depende de la magnitud de las vibraciones térmicas frente a la longitud de onda del rayo gamma. La medida de la proporción de eventos libres de retroceso proporciona información sobre la amplitud cuadrática media del movimiento térmico en el sólido. Entre las aplicaciones del efecto Mössbauer se cuentan también investigaciones en biofísica [Lang 1970], en la superficie del planeta Marte [Klingelhöfer 2002] y el estudio de la corrosión [Cook 2004].

Las interacciones magnéticas hiperfinas son muy útiles en el estudio de materiales magnéticos (ferromagnetos, ferrimagnetos y antiferromagnetos). La interacción hiperfina proporciona una medida indirecta de la magnetización de la red de iones magnéticos y se utiliza para revelar los detalles de las interacciones magnéticas y su dependencia con parámetro externos.

En este tema se produce la relación del Laboratorio de Electrónica con los grupos de Materiales Magnéticos y Materiales Nanoestructurados pertenecientes al Laboratorio de Barridos Térmicos Mössbauer del Departamento de Física de la Facultad de Ciencias Exactas, UNLP. Ambos laboratorios trabajan en colaboración hace varios años, habiéndose producido múltiples publicaciones en conjunto en este tema [Benedetti et al 1981, 1982] [Battaiotto et al 1994] [Martínez et al 1996] [Mendoza Zélis et al 2002, 2004] [Veiga et al 2002], incluyendo desarrollo de equipamiento y nuevas técnicas de medición. Los materiales usualmente estudiados son diferentes aleaciones metaestables y materiales magnéticos de base hierro. En este marco se han desarrollado las tareas involucradas en esta tesis.

Habiéndose introducido en este capítulo los principales aspectos y aplicaciones del efecto Mössbauer, se procede a continuación con una descripción detallada del instrumental de laboratorio utilizado en las diferentes variantes de este tipo de experimentos, junto con la propuesta para una nueva configuración que constituye el núcleo de esta tesis. 



\section{Capítulo 2 \\ Instrumentación Mössbauer}

En términos generales, existen dos tipos diferentes de experimentos que utilizan absorción y/ o emisión de radiación gama, los cuales son usualmente referidos como a) selección de altura de pulsos y b) determinación de coincidencias temporales. Las distintas variantes de espectrómetros Mössbauer pertenecen a la primera categoría. En ella se pone especial énfasis en la determinación de la energía de los eventos detectados, a expensas de la información relacionada con el tiempo en que dicha detección sucede. Los experimentos de coincidencias, en los cuales la información relativa al tiempo debe ser registrada, quedan fuera de los alcances de esta tesis.

La instrumentación nuclear se encuentra hoy bien asentada en el mercado. La mayoría de los diseños exitosos llevan varias décadas comercializándose y existen al menos dos fabricantes (Ortec y Canberra) que se disputan el liderazgo del mercado. El estándar NIM (por sus siglas en inglés Nuclear Instrumentation Module) data del año 1964. Fue diseñado específicamente para las comunidades de física de altas energías y nucleares. El objetivo fue promover un sistema de módulos de instrumentación intercambiables. En los experimentos Mössbauer se utilizan arreglos de módulos NIM para obtener la funcionalidad deseada. Los módulos y racks (también llamados bins) tienen especificaciones mecánicas y eléctricas normalizadas ${ }^{1}$.

En este capítulo se describen las dos variantes más utilizadas del espectrómetro Mössbauer de transmisión: el de aceleración constante y el de velocidad constante. Como se mencionó en el capítulo anterior, en la instrumentación de un espectrómetro Mössbauer pueden identificarse tres etapas fundamentales: detección, accionamiento y procesamiento. Tanto la técnica de aceleración constante como la de velocidad constante comparten las etapas de

1 Para una descripción completa del estándar NIM y otros componentes de instrumentación nuclear relacionados con la información de tiempo, puede el lector interesado referirse a la bibliografía especializada [Leo 1994] [Knoll 2000]. 
detección y accionamiento, diferenciándose en la etapa de procesamiento. Por lo tanto este capítulo está organizado en forma de presentar primero los detalles relativos a la instrumentación de las etapas comunes (detección y accionamiento), para luego mostrar las dos variantes de espectrómetro y sus aplicaciones, poniendo énfasis en la etapa de procesamiento.

A continuación de esta reseña de la instrumentación Mössbauer se presenta una propuesta para una tercera alternativa de funcionamiento: el espectrómetro de velocidad programable. La formalización de la técnica, el diseño de un espectrómetro y la implementación en laboratorio constituyen el tema de esta tesis. Esta nueva alternativa combina las técnicas de aceleración constante y de velocidad constante, con el objetivo de obtener un instrumento más eficiente y versátil. Para finalizar el capítulo se presentan los casos de aplicación del nuevo instrumento.

\subsection{La etapa de detección}

El objetivo de este bloque de instrumentación, presentado esquemáticamente en la Figura 6, es seleccionar los eventos de la energía en estudio (14.4 KeV para el hierro) e indicar cada ocurrencia con una señal lógica. Estas señales luego serán contadas y representadas en función de la velocidad de la fuente. Este bloque se compone básicamente de un detector de radiación con su respectiva fuente de alta tensión, un preamplificador seguido de un amplificador que conforma los pulsos de corriente provenientes del detector, y finalmente un discriminador diferencial que selecciona los pulsos según su energía (altura del pulso conformado por el amplificador), señalándolos con un pulso lógico.

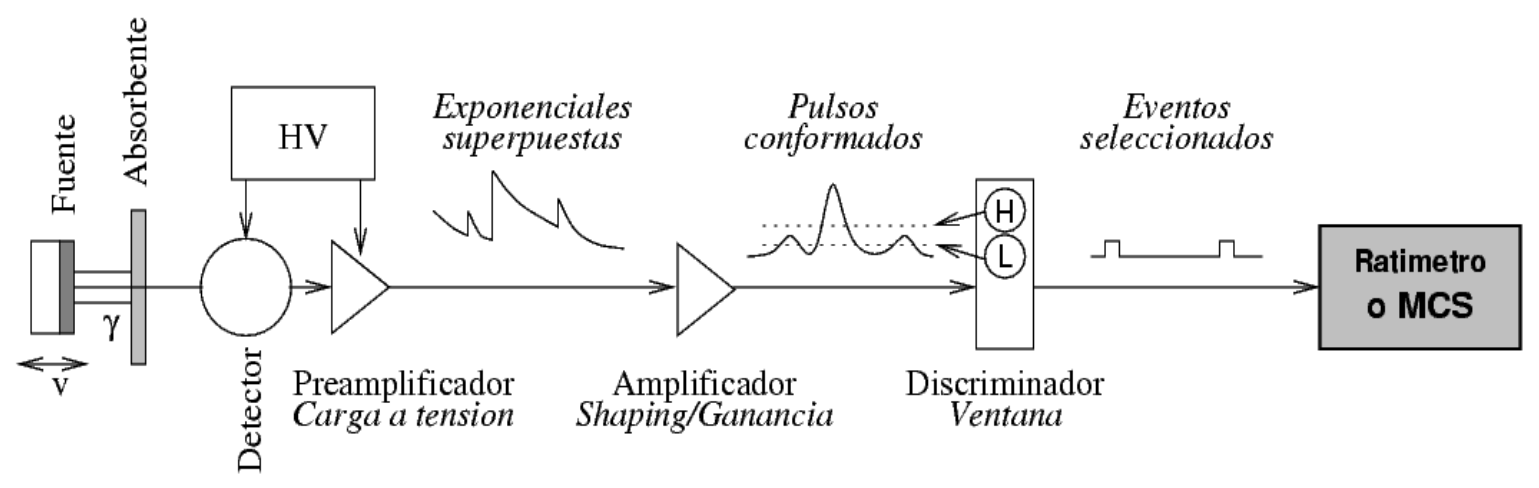

Figura 6: Componentes de la etapa de detección.

La cadena de detección, preamplificación y amplificación es típica en instrumentación nuclear. Es utilizada para obtener pulsos de altura proporcional a la energía del fotón detectado. Si se reemplazara el discriminador por un analizador multicanal, se obtendría un espectro de energía de la fuente.

Los criterios elementales de diseño de esta etapa se presentan a continuación, mostrando los aspectos relacionados con el caso particular del espectrómetro Mössbauer. En la bibliografía especializada [Leo 1994] [Knoll 2000] pueden encontrarse aspectos más generales respecto de esta configuración. 


\subsubsection{El detector}

Los tres tipos de detectores de radiación gamma de bajas energías que pueden utilizarse son (i) contadores proporcionales para energías menores a $40 \mathrm{KeV}$, (ii) centelladores para energías entre 50-100 KeV y (iii) detectores de estado sólido como $\mathrm{Si}(\mathrm{Li})$ o $\mathrm{Ge}(\mathrm{Li})$.

Los más utilizados para el estudio de materiales de base hierro (dada la energía de $14.4 \mathrm{KeV}$ en estudio) son del tipo contador gaseoso proporcional, por ser los más eficientes. Se trata de un recipiente metálico a potencial de tierra con un conductor central sometido a alta tensión, usualmente del orden de los $2 \mathrm{KV}$. El recipiente contiene criptón, xenón o argón, con una pequeña cantidad de metano como moderador, a presión atmosférica o superior. Los fotones de interés entran en la cámara a través de una ventana de mylar o berilio muy delgada, ionizando algunos átomos de argón. La creación de cada par ion/electrón consume aproximadamente $30 \mathrm{eV}$. Los iones positivos se desplazan a la pared exterior del detector y los electrones al hilo central. Los electrones generados ionizan más átomos, produciendo una avalancha muy próxima al hilo central con una ganancia proporcional de hasta $10^{6}$. En la Figura 7 pueden verse un diagrama esquemático y una vista de un detector de este tipo.
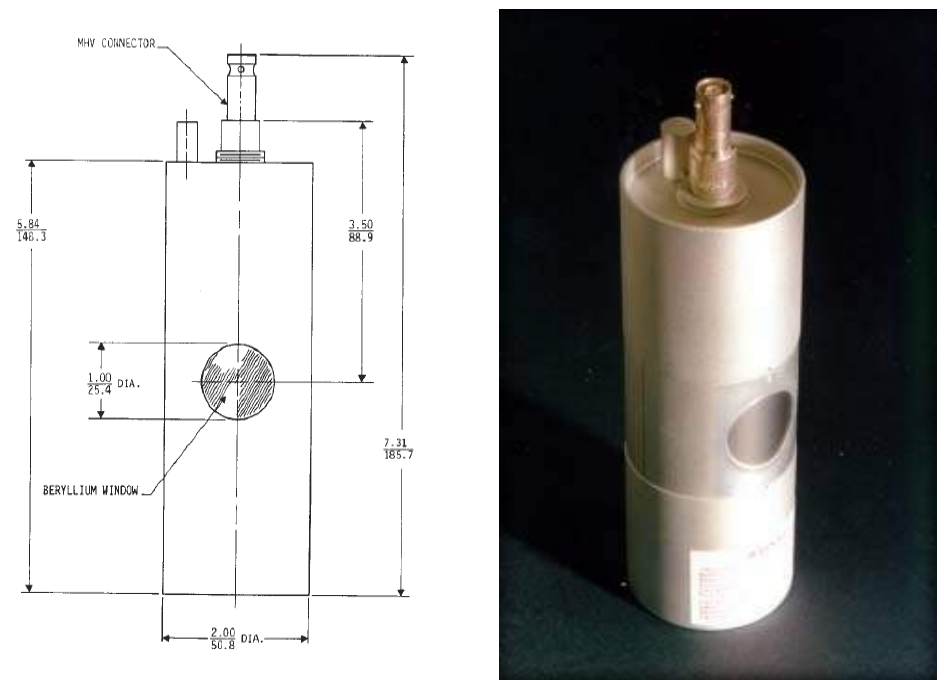

Figura 7: Detector tipo contador proporcional con ventana de berilio marca WissEl LND - 45431, ex FAST modelo 454X [http://www.fastcomtec.com]

Los fotones de 122 y $136 \mathrm{KeV}$ (en el caso del hierro) que entran al contador se dispersan, produciendo un amplio background de señales de baja energía. Si bien posteriormente un discriminador seleccionará las señales correspondientes a $14.4 \mathrm{KeV}$, aproximadamente el $20 \%$ de los pulsos que caen en la ventana del discriminador se deben a este efecto. Los pulsos de carga generados por el contador son manejados por un preamplificador que debe estar montado tan cerca del contador como sea posible. Finalmente los pulsos son enviados a un amplificador que los conforma adecuadamente, preservando la información de amplitud.

\subsubsection{El preamplificador}

La función básica de este dispositivo es amplificar las señales débiles provenientes del detector para que puedan ser transmitidas por un cable largo, manteniendo la relación señal a 
ruido lo más alta posible. A tal efecto el preamplificador se encuentra montado lo más cerca posible del detector para minimizar el largo del cable de entrada, reduciendo así su capacidad y el ruido por interferencia electromagnética.

La técnica más utilizada para la construcción de preamplificadores para contadores proporcionales es la del integrador de carga. Un amplificador con un capacitor de realimentación integra la señal proveniente del detector. La carga colectada en el capacitor es proporcional a la energía del fotón detectado. Una resistencia en paralelo con dicho capacitor produce la descarga exponencial del mismo, produciendo la salida que puede verse en la Figura 8. Una etapa de salida de cierta potencia le permite manejar cables largos.

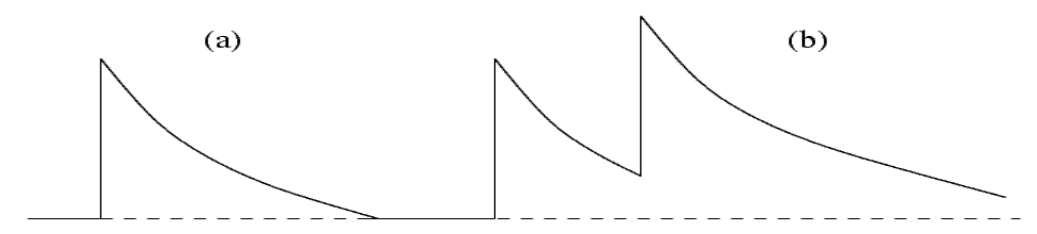

Figura 8: Salida en tensión del preamplificador: a) respuesta exponencial, y b) efecto de apilamiento en el caso de dos eventos idénticos próximos, resultando un pulso de altura mayor.

La constante de tiempo de descarga varía según el diseño pero es generalmente bastante grande (del orden de los 50 us o más). Esta no puede hacerse muy rápida pues se produciría una deformación en el flanco de subida de la exponencial, alterando la altura del pulso. Para obtener respuestas más rápidas suele reemplazarse la resistencia de descarga por un circuito de descarga manejado por la salida del comparador y acoplado ópticamente. En la Figura 9 se presenta un amplificador marca Canberra especialmente diseñado para este tipo de experiencias, junto con su diagrama esquemático. Estos equipos tienen un rise-time menor que $20 \mathrm{~ns}$ y operan con tensiones de hasta $3000 \mathrm{~V}$.

El tiempo de descarga del preamplificador es un factor crítico respecto de la eficiencia de la etapa de detección. Si dos fotones inciden en el detector separados por un tiempo menor que el tiempo de descarga del capacitor, se produce un efecto de apilamiento de los eventos, tal como se muestra en la Figura 8b. Este efecto produce un pulso de altura mayor a la real, y si los eventos van a ser discriminados por altura, un evento apilado será descartado a pesar de ser válido. La forma de evitar este efecto es restringiendo la tasa de arribo de los eventos (solución poco eficiente, ya que prolonga el tiempo de medida) o cambiándole la forma al pulso, siempre manteniendo la información de interés (en este caso la amplitud). Dicha conformación del pulso está a cargo del amplificador.
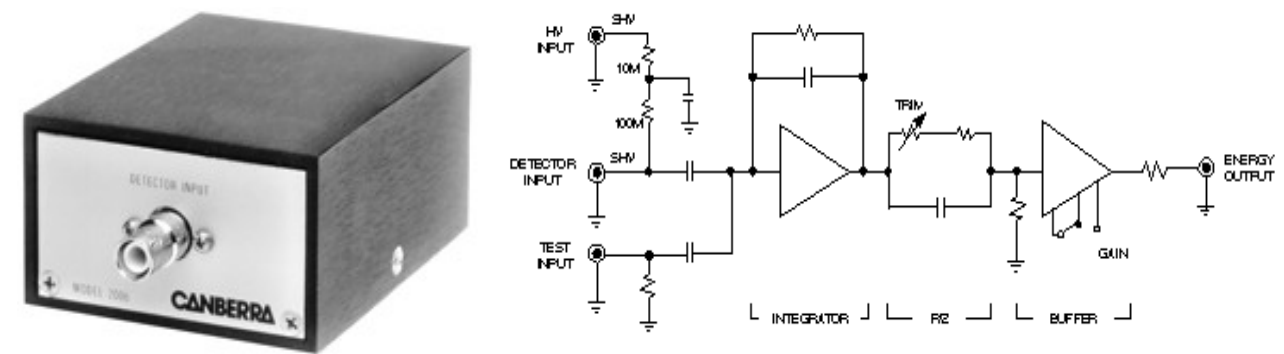

Figura 9: Preamplificador para contadores proporcionales marca Canberra modelo 2006: a) vista y b) diagrama esquemático. 


\subsubsection{El amplificador}

Este es un componente crítico de la etapa de detección, pues sus características (rango de ganancia, forma del pulso de salida, relación señal a ruido) pueden influir notablemente en la calidad de los resultados obtenidos. Además de amplificar la señal proveniente del preamplificador, este dispositivo tiene el objetivo primordial de conformar dichos pulsos para su posterior selección. El amplificador puede verse además como un procesador de señal. En el caso de la espectroscopia Mössbauer es fundamental que el pulso procesado mantenga la información de amplitud proporcional a la energía del fotón detectado.

Existen dos razones para cambiarle la forma al pulso. La primera es extraer la información necesaria de los pulsos que llegan apilados del detector, como muestra la Figura 10. Esto se logra mediante una diferenciación que debe tener algún mecanismo para restaurar la línea de base.
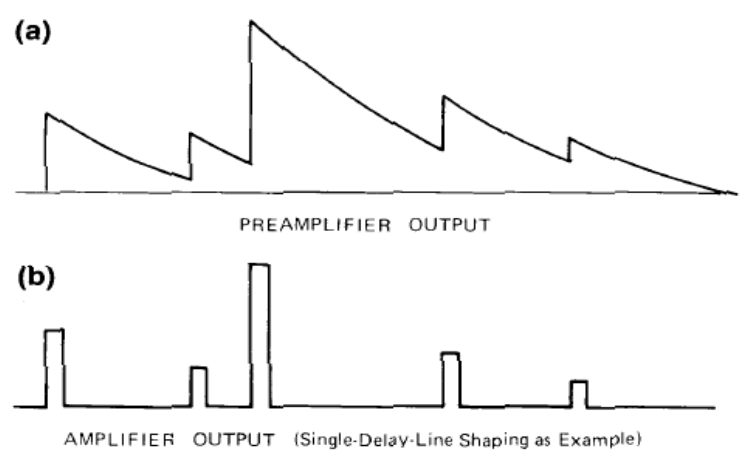

Figura 10: Conformación ideal de pulsos: a) salida del preamplificador, y b) salida del amplificador [EG \& G-Ortec Catalog]

La segunda razón para conformar los pulsos es darles una forma tal que sean más insensibles al ruido, por medio de sucesivas etapas de filtrado pasabajo. Figura 11. En esta etapa siempre existe el siguiente compromiso: para hacer angostos los pulsos de salida (que contienen la información de amplitud) y evitar así el apilamiento, es necesario que el amplificador disponga de una gran ganancia en alta frecuencia, con lo cual el ruido a la salida aumenta, desmejorando la resolución de amplitud.

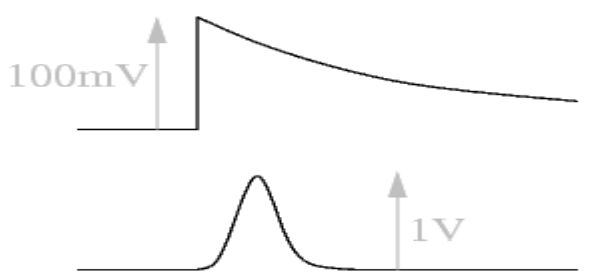

Figura 11: Entrada exponencial y salida pseudo gaussiana del amplificador.

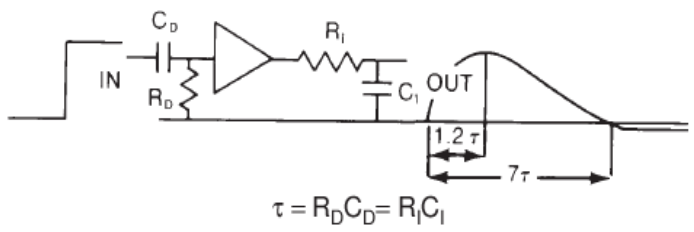

Figura 12: Conformación CR-RC con shaping $\tau$ [EG \& G-Ortec Catalog]. 
Si dos eventos suceden en un lapso de tiempo menor que el ancho del pulso de salida del amplificador, se producirá un efecto de apilamiento. En este caso serán seleccionados por el discriminador eventos que no corresponden a la altura correcta y se descartarán fotones de 14 $\mathrm{KeV}$. El resultado es que a la salida del discriminador disminuye la proporción de fotones con la energía correcta para producir absorción resonante, disminuyendo así el efecto y por lo tanto disminuyendo la relación señal a ruido del espectro. Debe tenerse en cuenta que en el amplificador aun no han sido discriminados los eventos de interés, por lo que el efecto de apilamiento es crítico en esta etapa, ya que la tasa es mucho mayor que la que luego será registrada en el espectro. A fin de minimizar el impacto del efecto de apilamiento, los fabricantes implementan circuitos de rechazo (pileup rejection). La técnica consiste en señalizar la ocurrencia de apilamiento, a fin de que el evento detectado no sea tenido en cuenta.

La conformación más simple es la llamada CR-RC de la Figura 12, en la cual se igualan las constantes de tiempo de la entrada y la salida (shaping time). En la Figura 13 se muestra una conformación más avanzada llamada pseudo-gaussiana [EG \& G Ortec Catalog]. Puede demostrarse que esta forma mejora la relación señal a ruido, resultando las formas de salida de la Figura 14. En este caso el ancho del pulso unipolar es 5 veces la constante de tiempo (7 para la conformación bipolar) y permanece constante para todas las amplitudes. En los amplificadores comerciales este ancho puede aumentarse en caso de requerirse mayor inmunidad al ruido, a expensas de aumentar la probabilidad de apilamiento.

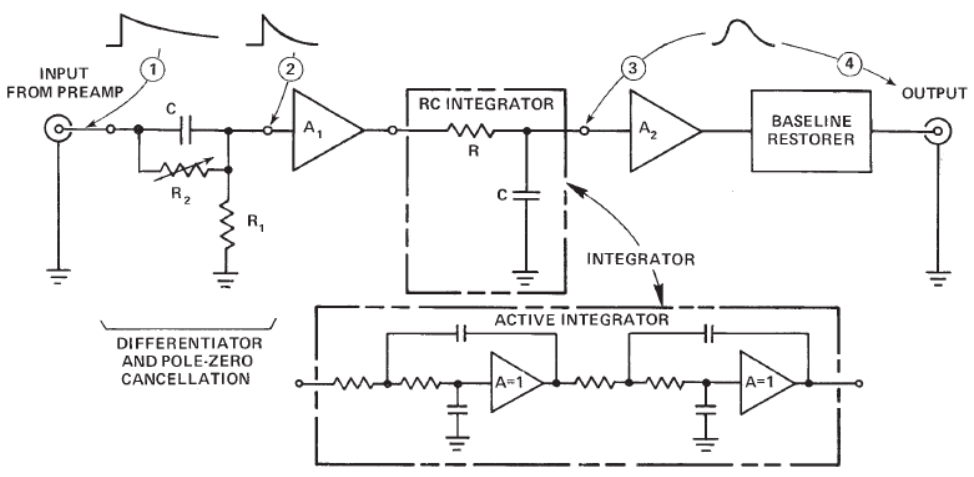

Figura 13: Conformación pseudo-gaussiana [EG \& G-Ortec Catalog].
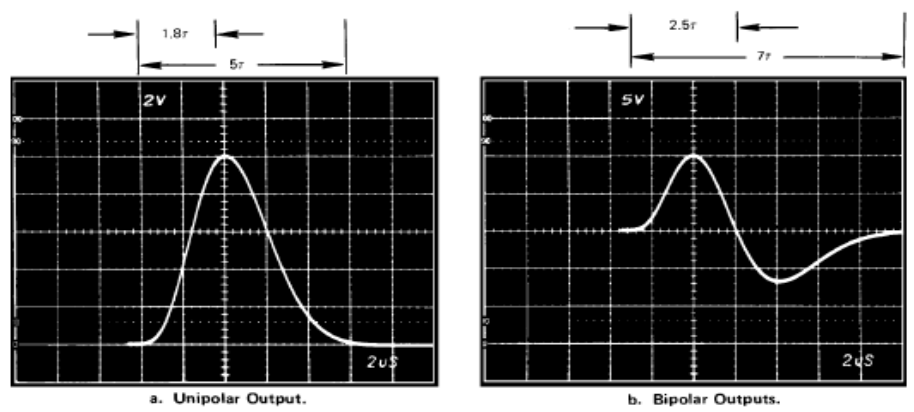

Figura 14: Salida a) unipolar y b) bipolar de un amplificador con conformación pseudo-gaussiana con un shaping time de 2 us [EG \& G-Ortec Catalog]. 
La restauración de la línea de base de la salida es un tema crítico para la medida de la altura del pulso. En este aspecto la salida bipolar es de implementación más simple, aunque resulta en un ancho de pulso mayor. Los fabricantes utilizan diversos circuitos para compensar este efecto, denominados BLR (baseline restorers).

Existen en el mercado diversos modelos de este tipo de amplificadores, entre los cuales pueden mencionarse los Canberra (modelos 2022 a 2026) y Ortec (modelos 572, 575 y 590).

En el Capítulo 3 se procede con un estudio más detallado de las consecuencias de la conformación de los pulsos en la performance del experimento y se propone un procedimiento de optimización para toda la etapa de detección.

\subsubsection{El espectro a la salida del amplificador}

Colocando la fuente sin absorbente y conectando un analizador multicanal a la salida del amplificador se obtiene un histograma de amplitudes que representa el espectro de potencia de la fuente tal como es registrado por el conjunto detector, preamplificador y amplificador. El espectro obtenido en la práctica es similar al presentado en la Figura 15.

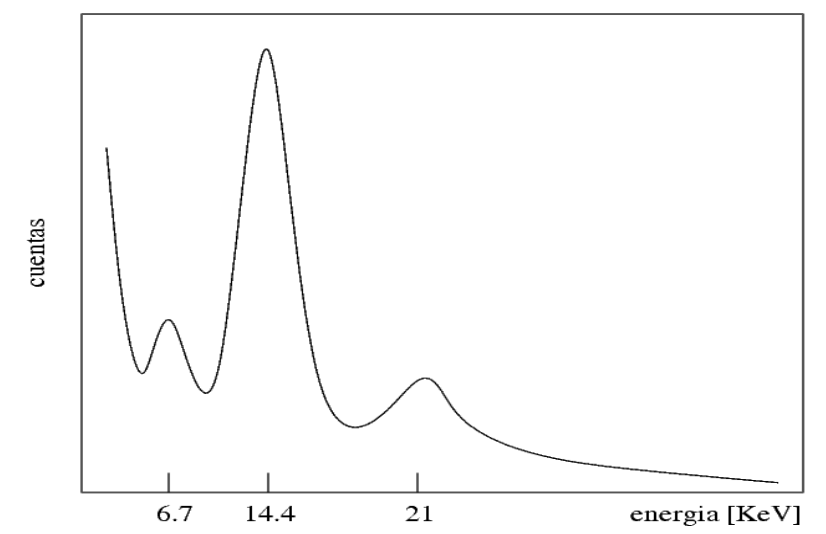

Figura 15: Espectro de amplitudes a la salida del amplificador.

Esta distribución de energías difiere mucho de la distribución teórica que puede deducirse del modelo de transiciones nucleares presentado en la Figura 3. De ese modelo simple solo podemos esperar transiciones de 136, 122 y $14.4 \mathrm{KeV}$ con un ancho de línea del orden de los $\mathrm{neV}$, las cuales producirían líneas de un solo canal de ancho en el espectro obtenido por el multicanal. A continuación se enumeran los fenómenos presentes en el proceso de detección que apartan la forma del espectro resultante en la práctica de este modelo ideal:

i. Las líneas de 122 y $136 \mathrm{KeV}$ no son detectadas pues la ganancia de los detectores proporcionales es muy baja en tan altas energías.

ii. La línea de $14.4 \mathrm{KeV}$ tiene un ancho del orden de los $\mathrm{KeV}$ en lugar de de los neV del ancho teórico. Esto se debe a la geometría del detector. No todos los rayos gamma incidentes tienen el mismo recorrido dentro del detector (si bien todos tienen la misma energía), produciendo diferentes cantidades de carga depositada. En este punto también contribuyen las imprecisiones del amplificador y del preamplificador durante el tratamiento de los pulsos, resultando en un ancho de línea muchos órdenes mayor 
que el teórico.

iii. Aparecen dos líneas de energía de menor amplitud, dentro del rango de sensibilidad del detector. La de mayor energía (aproximadamente $20 \mathrm{KeV}$ ) se debe un rayo X que es emitido durante interacciones producidas entre el Co57 y la matriz de Rodio en la que éste está insertado. La línea de menor energía $(6.7 \mathrm{KeV})$ se origina también debido a un rayo $\mathrm{X}$ que es emitido cuando uno de los electrones de las capas exteriores del Fe57 cae a una de las capas interiores para compensar el electrón que fue absorbido por el núcleo en la transición de Co57 a Fe57. Estas líneas también están ensanchadas, debido a los motivos expuestos en el punto anterior.

iv. El ruido eléctrico de la parte analógica de la etapa de detección se manifiesta en la zona de bajas amplitudes, aumentando al acercarse al origen del espectro. Los espectros experimentales muestran un recorte en esta zona, ya que a fines prácticos debe utilizarse un discriminador a la salida del amplificador, para no saturar el sistema de conteo con estas señales muy abundantes de baja amplitud.

v. Si bien los rayos gamma de 122 y $136 \mathrm{KeV}$ no son detectados, si lo es el fondo debido al efecto Compton asociado a estas energías, el cual se manifiesta como una meseta en la zona de energías de las decenas de KeV. Esta meseta es más elevada en la zona de bajas energías debido a la variación de ganancia del detector.

vi. La radiación de fondo, si bien existe, es despreciable en cuanto a estadística ante la presencia de una fuente radiactiva.

En la próxima sección se mostrará cómo se utiliza un discriminador diferencial para seleccionar sólo los eventos de este espectro que tienen una cierta probabilidad de ser absorbidos resonantemente, o sea aquellos descriptos en el punto ii.

Al colocar un absorbente, la estadística baja en todo el rango de energía. Al producirse absorción resonante la amplitud relativa del pico de $14.4 \mathrm{KeV}$ disminuye,

Debe aclararse que al modificar la velocidad de la fuente, sólo se aumenta o disminuye la energía de los fotones en el orden de los micro eV, por lo cual el efecto no es percibido en este espectro de amplitudes que tiene $\mathrm{KeV}$ de rango.

En cambio, los efectos de la temperatura son visibles. Al aumentar la temperatura del detector, aumenta su ganancia, produciendo exponenciales de mayor amplitud para eventos de la misma energía. Se produce, entonces, un desplazamiento hacia la derecha del espectro, originando corrimientos relativos de la ventana de selección que se describe a continuación.

\subsubsection{El discriminador diferencial}

Este dispositivo, llamado también SCA (por sus siglas en inglés Single Channel Analyzer), es básicamente un selector de altura de pulsos que genera una salida lógica cada vez que recibe en su entrada un pulso de una altura menor que un máximo y mayor que un mínimo, regulables ambos. Funciona como selector de energías si los pulsos tienen una altura proporcional a la energía de los fotones detectados, como es el caso de la etapa de detección descripta.

Al procedimiento de selección de los fotones de interés mediante los niveles máximo y 
mínimo del discriminador se lo denomina usualmente "ventaneo". Este se realiza manualmente utilizando diferentes técnicas. Una de ellas es utilizando un analizador multicanal (MCA) conectado directamente a la salida del amplificador, relevando un espectro de energía completo. Esta técnica permite seleccionar por inspección los niveles alto $(\mathrm{H})$ y bajo (L) de discriminación, como muestra la Figura 16. Esta técnica insume bastante tiempo, por lo que en la práctica el multicanal es reemplazado por un osciloscopio con persistencia. Los niveles del discriminador pueden ajustarse disparando los pulsos de entrada con la salida del discriminador y ajustando la persistencia en forma adecuada. Esta técnica es poco precisa y depende de la habilidad y experiencia del operador.

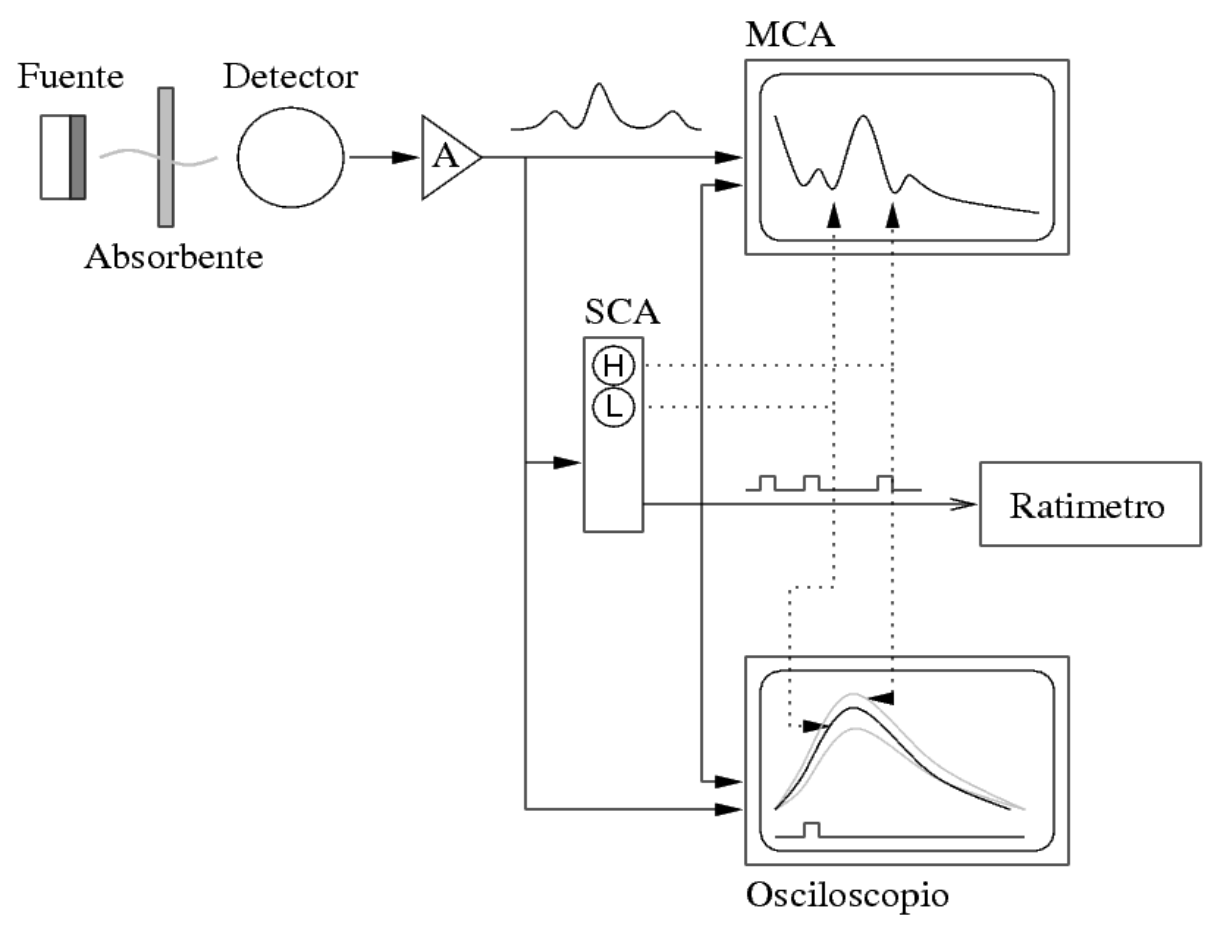

Figura 16: Diferentes posibilidades para el ventaneo: a) con multicanal, y b) con osciloscopio.

Si no se intenta preservar información precisa acerca del de tiempo de ocurrencia del evento, el diseño del discriminador es relativamente simple. Puede construirse con dos comparadores rápidos junto con alguna lógica adicional, utilizando la derivada del pulso de entrada para detectar el máximo. Un diseño propio basado en el comparador LM360 se presenta en el Apéndice A. Este tipo de diseño presenta la desventaja de que el retardo del pulso de salida es proporcional a la altura del pulso de entrada, con lo cual se destruye la información relativa al tiempo de ocurrencia del evento (efecto denominado "walk"). Para los experimentos en los cuales dicha información es importante, como en el caso de la determinación de coincidencias, suele utilizarse una técnica denominada "constant-fraction". En ésta, por medio de un procesamiento analógico de la señal se consigue producir el pulso de salida luego de un tiempo determinado por un porcentaje del rise-time del pulso de salida del amplificador, el cual es constante [Leo 1994]. Estos dispositivos se denominan Timing SCA. Para la construcción de un espectrómetro Mössbauer no es indispensable la utilización de un discriminador de este tipo. Pueden utilizarse tanto los diseños derivadores como los constant- 
fraction.

Los módulos NIM comerciales tienen generalmente dos tipos de salidas: la salida "lenta" es un pulso TTL de $500 \mathrm{~ns}$ de ancho, con un rise-time menor que 20 ns y una resolución entre pulsos de 800 ns; la salida "rápida" utiliza el estándar NIM, produciendo un pulso de corriente negativo de $20 \mathrm{~ns}$ de ancho, rise-time menor que $5 \mathrm{~ns}$ y una resolución de pulsos de 200 ns. Estas características determinan el tiempo muerto del discriminador, limitando la tasa de conteo que puede manejar.

El discriminador cierra la etapa de detección, suministrando una tasa de pulsos lógicos que se verá afectada ante la presencia de resonancia Mössbauer. La etapa de procesamiento es la encargada de construir el espectro de absorción registrando esta tasa en función de la velocidad relativa de la fuente radiactiva.

\subsection{La etapa de accionamiento}

A diferencia de la etapa de detección, que tiene características similares para un gran número de experimentos de física nuclear, este bloque de instrumentación es exclusivo de los espectrómetros Mössbauer. La fuente radiactiva debe desplazarse a una velocidad bien controlada a fin de conocer con precisión la energía de los fotones emitidos. El recorrido de la fuente debe ser cíclico y de valor medio nulo, a fin de no producir desplazamientos acumulativos. En la Figura 17 se presenta un diagrama esquemático de esta etapa.

La evolución temporal del desplazamiento determina las características del experimento, cuyas variantes se describen más adelante. En esta sección se mostrarán los transductores utilizados y se comentarán brevemente las técnicas usualmente empleadas para controlar su funcionamiento. En el Capítulo 4 se tratan estos temas con mayor profundidad y se presentan nuevas técnicas para un funcionamiento adecuado a velocidad constante.

No es intención de esta tesis profundizar en los detalles mecánicos de los transductores. Sólo se tratarán los mismos en relación con los parámetros eléctricos y su influencia sobre el lazo de control. Estos dispositivos se encuentran disponibles en los laboratorios de espectroscopia Mössbauer, siendo el componente más costoso del instrumento. Por lo tanto el tratamiento de este tema estará orientado más a la optimización del uso de los transductores que a la posible modificación de los mismos.

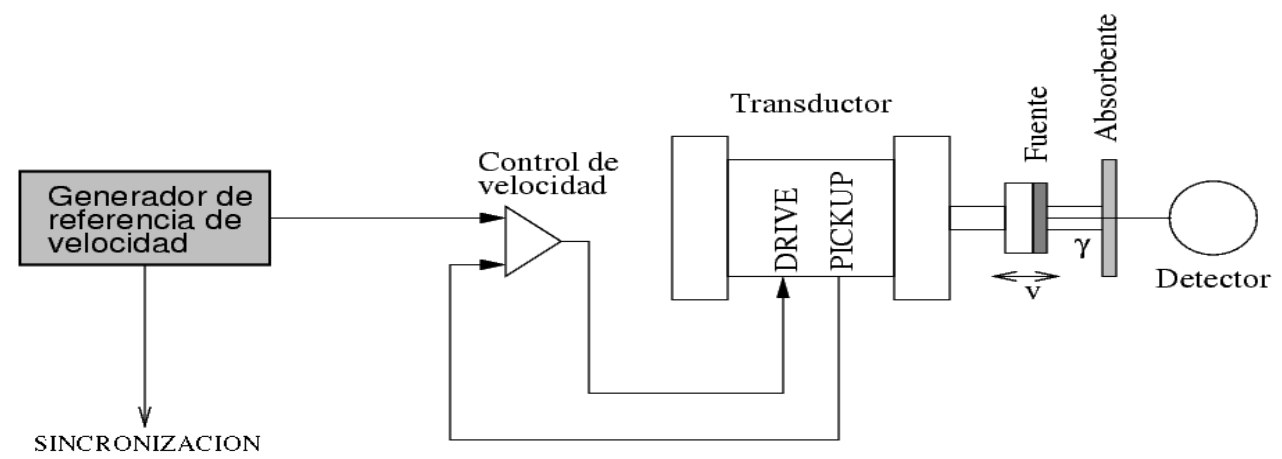

Figura 17: Componentes de la etapa de accionamiento. 


\subsubsection{El transductor}

Existen tres tipos de transductores que pueden utilizarse para accionar sobre la velocidad de la fuente radiactiva en una experiencia Mössbauer. Estos pueden ser (i) mecánicos, (ii) piezoeléctricos, o (iii) electromecánicos.

Luego de un tiempo de ser utilizados, los transductores mecánicos del tipo rotante [Mössbauer 1961] o péndulo [Flinn 1963] fueron abandonados debido a las complicaciones en su operación y las dificultades que presentan cuando se desean automatizar las experiencias.

Existen trabajos publicados recientemente que utilizan cristales piezoeléctricos apilados (stacks) como transductores de velocidad [Casas et al 2002]. Estos tienen el inconveniente de que requieren valores de tensión del orden de los KV. La alta frecuencia de resonancia de dichos cristales y el limitado recorrido que puede obtenerse imponen severas limitaciones en la electrónica asociada. Por ello, su utilización en experiencias de laboratorio es muy poco frecuente, reservándose para casos en que la miniaturización es un factor de diseño predominante.

En la primera mitad de la década del '60 se perfeccionó un transductor electromecánico para uso específico en experiencias Mössbauer [Rubin 1962] [Cohen 1963] que fue finalmente descripto por Kankeleit tal como se presenta en la Figura 18 [Kankeleit 1964]. Desde entonces ha experimentado sólo algunas pocas modificaciones importantes en su diseño [Ruegg et al 1965] [Zane 1966] [Carmeliet 1968] [Overbeeke 1971] [Genard-Riondet et al 1978] [Lierop 2001] [Ponikvar 2002] [Kluger 2003] [Mashlan 1999, 2006]. Este tipo de transductor se ha transformado en el modelo más utilizado en la actualidad para experiencias de laboratorio.

El principio de funcionamiento es simple y se asemeja al de un doble parlante magnético de audio. Un eje rígido está montado horizontalmente sobre una suspensión a resortes que le permite realizar desplazamientos solamente a lo largo del mismo. El eje tiene montada en un extremo la fuente radiactiva. Solidaria a este eje se encuentra la bobina de accionamiento (DRIVE), que se mueve dentro de un campo magnético uniforme provisto por un imán permanente. Al circular corriente por la bobina se produce el desplazamiento de la misma, arrastrando al eje y a la fuente.

También solidaria al eje se encuentra otra bobina (VEL o PICKUP) que también se mueve dentro de un segundo campo magnético. En esta bobina se induce una tensión proporcional a la velocidad de desplazamiento de la misma y por lo tanto proporcional a la velocidad de la fuente. Esta tensión se utiliza como realimentación de velocidad. Debe notarse que esta realimentación es una tensión proporcional a la velocidad, no existiendo en la misma información respecto de la posición absoluta del eje. Esto hace que deban tomarse precauciones respecto del valor medio y drift de la referencia de velocidad.

En la Figura 19 se muestra un transductor electromecánico marca ASA modelo K3 (fabricación discontinuada) y en la Figura 20 uno más actual marca WissEl modelo MA 260, ambos disponibles en nuestros laboratorios.

La rigidez del transductor es un factor importante, a fin de evitar resonancias mecánicas. La linealidad estará determinada por la uniformidad de los campos magnéticos, la tensión de los 


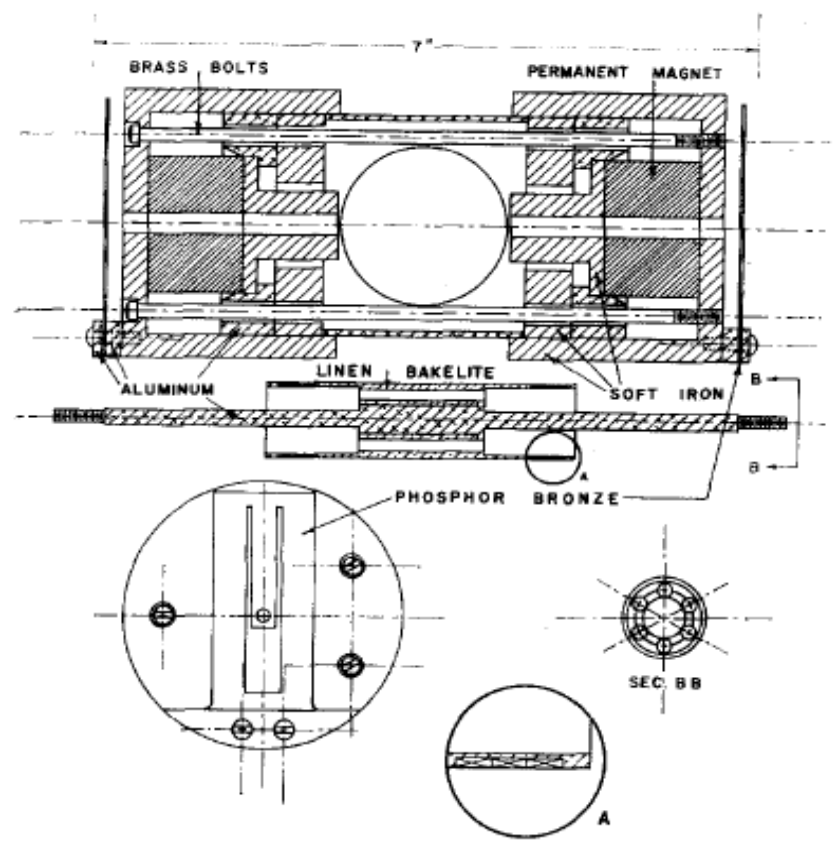

Figura 18: Detalles del transductor electromecánico descripto por Kankeleit en 1964.
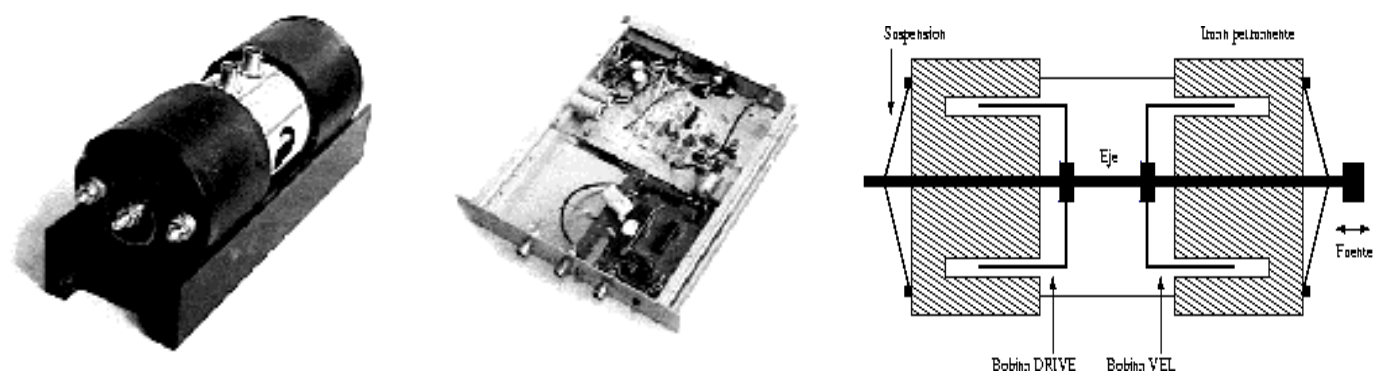

Figura 19: a) Vista del transductor marca ASA modelo K3. b) Unidad de control de velocidad desarrollada en nuestros laboratorios [Benedetti et al 1982], montado en un módulo NIM. c) Diagrama esquemático del transductor.

resortes de suspensión y el rozamiento.

El montaje mecánico del conjunto transductor, muestra y detector es un punto crítico del experimento. Debe cuidarse que el movimiento del transductor no se transmita hacia la fuente a través de vibraciones del conjunto, para lo cual suele montarse solidario a una base rígida de cemento que no oscile a la frecuencia de trabajo del transductor.

\subsubsection{El control de velocidad}

Para controlar la velocidad del transductor electromecánico se utilizan generalmente sistemas analógicos realimentados que emplean diversas técnicas de compensación [Zane 1966] [Chase 1969] [Gupta 1973] [Corson 1980]. La topología de realimentación es similar en todos los casos: la tensión proporcional a la velocidad de la fuente entregada por el transductor (PICKUP) se compara con una referencia de velocidad en tensión para generar la señal de error. Con circuitos eléctricos basados en operacionales se agregan ganancia y 

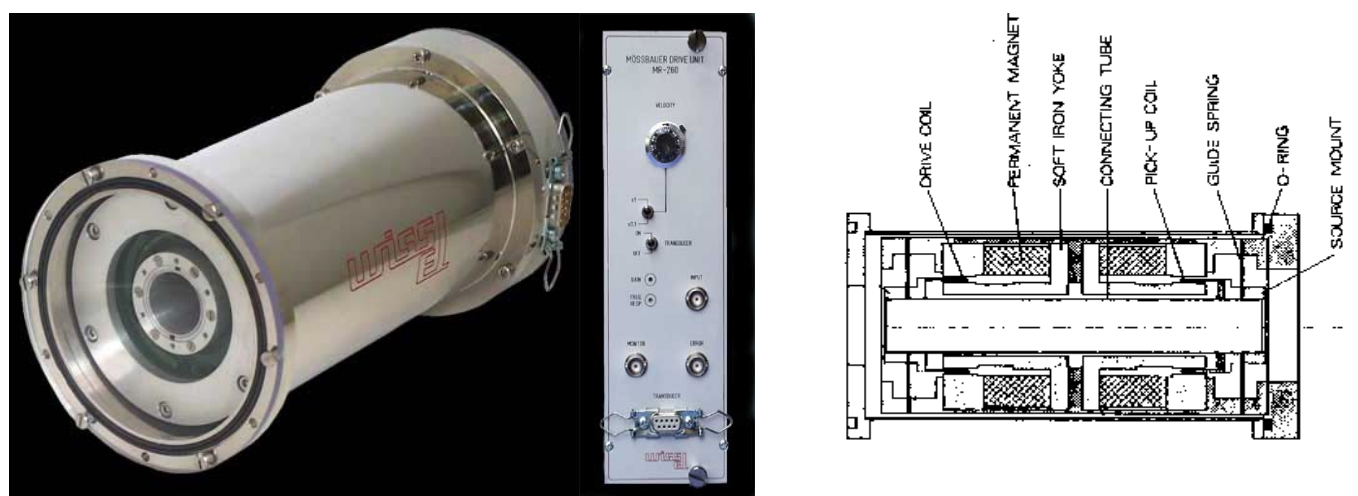

Figura 20: Mössbauer Drive System 260, WissEl Scientific Instruments: a) transductor electromecánico, $b$ ) control de velocidad en formato NIM, y c) esquemático del transductor.

compensaciones, y finalmente una etapa de potencia se encarga de accionar sobre el DRIVE del transductor. En el capítulo 4 se presentan las particularidades de este tipo de sistemas realimentados, junto con las técnicas de control más utilizadas, a modo de introducción de los aportes de esta tesis en el tema.

En general el mismo fabricante del transductor proporciona un control de velocidad adecuado para el mismo, capaz de manejar la resolución en velocidad necesaria para este tipo de experimentos (típicamente $0.02 \mathrm{~mm} / \mathrm{s}$ ), como así también el rango de velocidades que suelen ser utilizadas (al menos -10 a $10 \mathrm{~mm} / \mathrm{s}$ para el estudio de Fe). Estos dispositivos incluyen una etapa de potencia según los requerimientos del transductor, entrada de referencia externa y salidas para observar la referencia de entrada (monitor) y el error. Las técnicas de compensación utilizadas usualmente en los equipos comerciales requieren una sintonización manual cuidadosa, la cual es muy sensible al contenido armónico de la referencia de velocidad. Este aspecto hace que las modificaciones en la referencia deban realizarse con un posterior ajuste, limitando las posibilidades de automatización.

La forma de la referencia de velocidad utilizada depende del tipo de experimento, según se mostrará a continuación. La tensión de referencia es suministrada por la etapa de procesamiento, la cual incluye un generador de señal adecuado. En las próximas secciones se muestran las dos variantes clásicas de la etapa de procesamiento utilizadas para la construcción de un espectrómetro de aceleración constante y uno de velocidad constante. Ambas implementaciones utilizan la etapa de accionamiento descripta en esta sección y la etapa de detección descripta en la sección anterior.

\subsection{El espectrómetro de aceleración constante}

Resumiendo lo expuesto hasta ahora, en un espectro Mössbauer se busca caracterizar la absorción del material como una función de la energía de los rayos gamma incidentes. La variación de energía se obtiene por efecto Doppler, donde el movimiento de la fuente de rayos gamma produce una variación de la frecuencia del fotón y por lo tanto de su energía. Entonces, obtener un espectro Mössbauer equivale a contar los fotones que atraviesan el material en función de la velocidad de la fuente. 


\subsubsection{Principio de funcionamiento}

El mecanismo más empleado en laboratorios para obtener un espectro para un determinado rango de velocidades es utilizando las etapas de detección y accionamiento descriptas anteriormente, en conjunto con una etapa de procesamiento compuesta por un generador de funciones y un multiescalímetro (Multi Channel Scaler o MCS) ${ }^{2}$ [Woodhams 1967] [Gaitanis 1969] [Player 1978] [Pourcell 1985] [Jing 1992] [Ericson 1994] [Blois 1998] [Woodhams 1991, 2001] [Velázquez 2005]. La Figura 21 muestra un diagrama en bloques de una implementación clásica. Esta configuración es muy utilizada en laboratorios dada su simple implementación y eficiencia. La etapa de detección, compuesta por el detector, el preamplificador, el amplificador y el discriminador fue descripta en detalle en la Sección 2.1. Los detalles pertinentes a la etapa de accionamiento, compuesta por el transductor electromecánico y su control de velocidad, se presentaron en la Sección 2.2. En este caso, la etapa de procesamiento contiene dos partes que se describen a continuación.

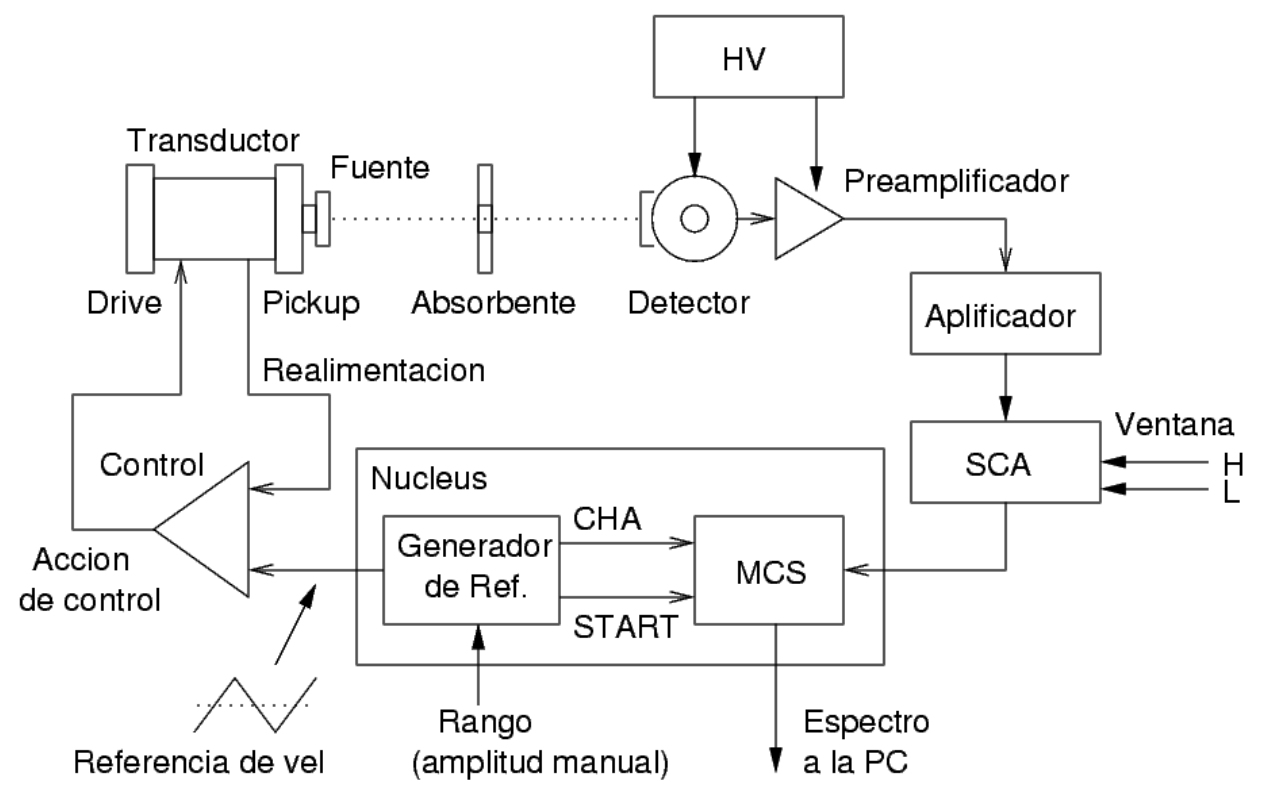

Figura 21: Diagrama en bloques de un espectrómetro Mössbauer de aceleración constante.

\subsubsection{El generador de referencia y el multiescalímetro}

La fuente de rayos gamma se encuentra montada sobre el transductor electromecánico, cuyo movimiento produce el barrido en velocidad. La fuente es desplazada cíclicamente de forma tal que un determinado rango de energías de interés es recorrido varias veces por segundo con valor medio nulo para no producir desplazamientos acumulativos. Un generador de funciones especialmente diseñado produce la referencia de velocidad periódica y el direccionamiento del canal activo del multiescalímetro. Estos equipos disponen de entradas digitales TTL de

2 Algunos analizadores multicanal (Multi Channel Analyzer o MCA) pueden operarse en modo MCS, como funcionalidad adicional. 
START (posicionamiento en la primer dirección de memoria, realizada una vez por ciclo) y CHA (avance a la próxima dirección, realizada $\mathrm{N}$ veces por ciclo, dependiendo del número de canales utilizados), de manera de sincronizar la referencia de velocidad con la conmutación de la memoria. Resulta entonces que el número de canal es una función de la velocidad a la que se mueve la fuente, y por lo tanto de la energía de los fotones. En otras palabras, los pulsos contados mientras la fuente está a una determinada velocidad siempre son guardados en la misma posición de memoria del multiescalímetro. El funcionamiento esquemático se detalla en la Figura 22.

En el caso de no disponerse de un multiescalímetro, puede utilizarse una técnica poco usual que consiste en modular la altura de los pulsos provenientes del discriminador con la forma de onda del oscilador, para luego enviarlos a un multicanal operando en modo analógico.

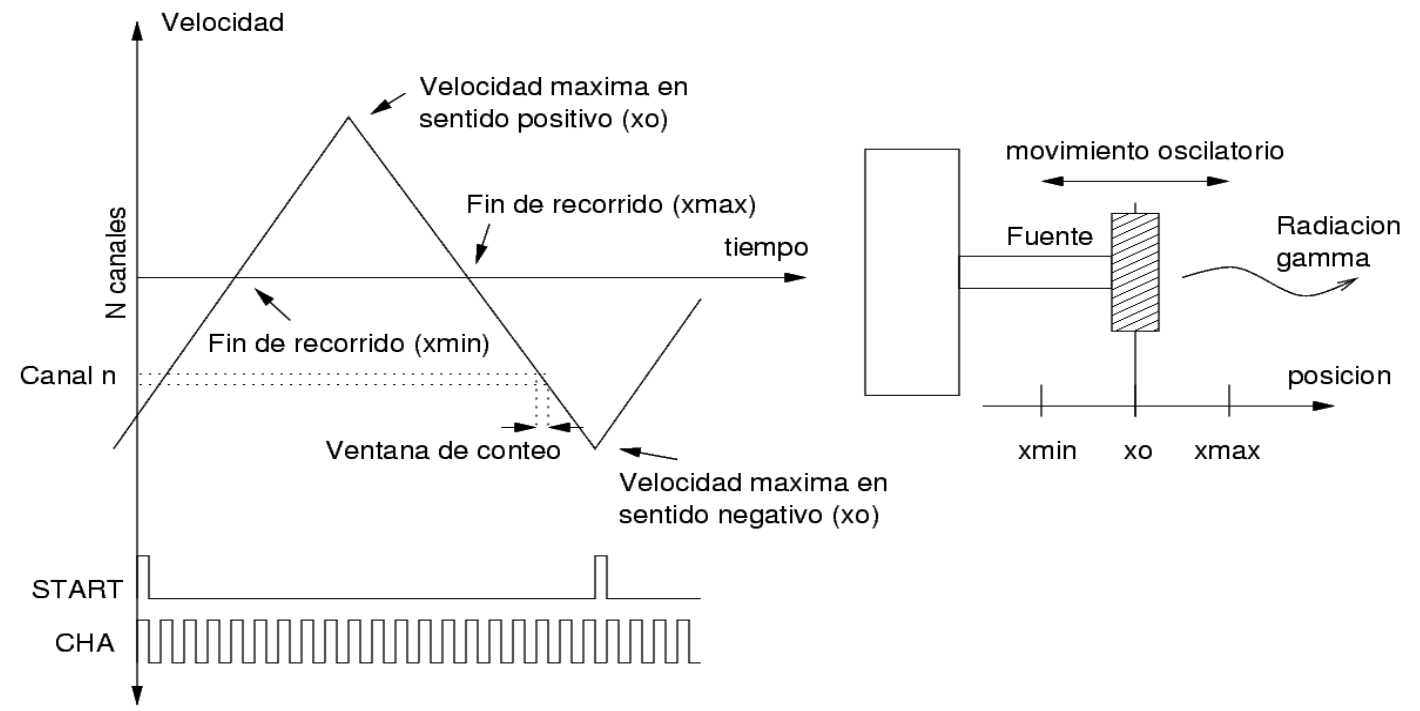

Figura 22: a) Referencia de velocidad para un espectrómetro Mössbauer de aceleración constante. b) Movimiento longitudinal de la fuente radiactiva. El punto $x_{0}$ corresponde a la posición de reposo del eje del transductor. Los puntos $x_{\max }$ y $x_{\min }$ corresponden al centro del espectro $(v=0)$ y $x_{0}$ a los extremos $\left(v_{\max }\right)$.

La salida del generador de funciones debe ser una tensión con valor medio nulo que se aplica a la entrada de referencia del control de velocidad. Si los incrementos de velocidad respecto del tiempo de conteo son constantes ( $\mathrm{dv} / \mathrm{dt}$ constante) se trata de un espectrómetro de aceleración constante. En este caso la referencia de velocidad es una rampa como la presentada en la Figura 22 y la escala de velocidad resultante es lineal. Así la gráfica del conteo versus el número de canal resultará adecuadamente ordenada para su análisis, salvando el hecho que cada velocidad es observada dos veces por ciclo, una vez a la ida y otra vez a la vuelta. Por lo tanto el espectro resultante debe "plegarse" para obtener el resultado final. Si bien esto puede parecer inconveniente, resulta de utilidad para verificar el buen funcionamiento del instrumento comparando las dos mitades.

Una referencia de velocidad en forma de diente de sierra evitaría repetir las velocidades, pero se descarta por el abrupto retorno que dificulta el funcionamiento del control de velocidad. Debe aclararse aquí que sería más natural producir la oscilación del transductor con una referencia de velocidad de forma sinusoidal. Pero en este caso resultaría un espectro difícil de 
visualizar, donde la mayoría del tiempo y la mayor parte de los canales son utilizados en los extremos de velocidad, donde el espectro contiene poca información. La forma de onda triangular resulta un compromiso adecuado.

El espectro resultante estará siempre centrado en velocidad nula y por lo tanto el rango de velocidades medidas será simétrico (deben medirse tanto velocidades positivas como negativas). Dicho rango se selecciona atenuando o amplificando la salida del generador de referencia.

El tiempo de la ventana de conteo por canal está impuesto por la frecuencia de trabajo del transductor (que en general se busca que se encuentre cerca de la resonancia del mismo) y el número de canales que se desea para el espectro. La potencia necesaria para controlar la velocidad será menor cuanto más cerca se encuentre la frecuencia fundamental de operación de dicha frecuencia de resonancia, como se mostró en la sección anterior.

Existen en el mercado generadores de referencia diseñados específicamente para este tipo de experiencias, como el modelo DFG-500 de WissEl. Todos ellos incluyen las señales lógicas de START y CHA para accionar el multiescalímetro, selección de la frecuencia en un amplio rango, selección del número de canales (cantidad de pulsos de CHA entre dos pulsos de START). Usualmente permiten la selección entre formas de referencia triangular o sinusoidal.

En los laboratorios de espectroscopia Mössbauer suele disponerse de equipos que integran el oscilador y el multiescalímetro en un único instrumento (unidad de procesamiento completa). En los laboratorios es frecuente encontrar una placa de expansión para PC marca Nucleus modelo MCS-II, en la que la referencia de velocidad triangular es generada digitalmente mientras internamente se produce la selección del canal.

\subsubsection{Puntos críticos y limitaciones de la instrumentación a aceleración constante}

Para una experiencia típica, la ventana de conteo del multiescalímetro será de algunas décimas de milisegundo. El valor exacto estará fijado por la frecuencia de operación del transductor y el número de canales. El número de eventos en dicha ventana es bajo, lo que obliga a que la lógica asociada a la base de tiempo y al contador deban operar a altas frecuencias y con precisión adecuada. Por ejemplo, si el instrumento opera con 256 canales y barriendo el rango de velocidades a $12 \mathrm{~Hz}$, resulta una ventana de $1 /(12 \mathrm{~Hz} .2 .256)=160 \mathrm{us}$. Si la tasa de conteo es de 20000 cuentas por segundo, resulta que en cada ventana se cuentan sólo 1 o 2 eventos en promedio. Para no introducir errores, el tiempo muerto del multiescalímetro (tiempo necesario para producir el cambio de canal) debe ser menor que el tiempo muerto de la etapa de detección.

Otro aspecto fundamental de esta configuración es la relación lineal que debe mantenerse entre el incremento del canal y el incremento de la velocidad, para todo el rango de operación. En este punto debe tenerse en cuenta tanto la calidad de la referencia triangular como el error introducido por el control de velocidad en todo el rango. Tal como se mostró en la Sección 2.2, no son admisibles frecuencias muy bajas de operación debido a la supresión de continua que es necesaria en la referencia para evitar desplazamientos acumulativos de la 
fuente radiactiva.

Finalmente debe tenerse en cuenta que son necesarias varias horas de medición para obtener un espectro Mössbauer con una relación señal/ruido aceptable. Sin embargo, no siempre es necesario un espectro completo para obtener la información buscada. Un ejemplo es el caso en que se desee observar la posición de una determinada línea de absorción y su evolución con un parámetro externo. En este experimento particular sería suficiente medir en el entorno de la energía de interés. Pero si se utiliza la técnica de aceleración constante el resultado es siempre un espectro centrado en velocidad nula. Es en este tipo de experiencias donde resulta útil la técnica de velocidad constante que se describe a continuación.

\subsection{El espectrómetro de velocidad constante}

En este caso se busca mantener constante la velocidad de la fuente de rayos gamma (fotones de energía constante) en una línea del espectro que se desee estudiar en particular, produciendo el barrido de algún factor externo, como por ejemplo la temperatura a la que se encuentra el material en estudio o el campo magnético externo al cual está sometido el mismo. Se produce así un histograma de la transmisión en función del parámetro externo para sólo una de las líneas de energía del espectro Mössbauer. El mecanismo usual en estos casos es producir un barrido en el tiempo del parámetro en estudio, al mismo tiempo que se registra la absorción [Sarma 1979] [Seberini 1988].

Si se desea estudiar el material en un rango de energías, o en varias energías no consecutivas, el experimento debe repetirse para cada una de ellas. Por medio de este experimento se pueden localizar rápidamente cambios en la transmisión debido a transformaciones de fase (estructurales o magnéticas) y reacciones sólido-sólido o sólido-gaseoso [Mendoza Zélis et al 2002].

En el Laboratorio de Electrónica del Departamento de Física fue desarrollado un instrumento que permite realizar esta experiencia con cierto grado de automatización [Veiga et al 2002]. Por supuesto que el mismo resultado puede obtenerse realizando un espectro de aceleración constante para cada valor de interés del parámetro externo [Chien 1978]. Pero esta técnica requiere de enormes tiempos de medida, produciendo información redundante.

\subsubsection{Principio de funcionamiento}

Si bien este experimento puede parecer más simple, presenta mayores complicaciones respecto de la instrumentación, sobre todo en la etapa de accionamiento. Para la etapa de detección pueden utilizarse los mismo componentes que en las experiencias de aceleración constante (conjunto detector, preamplificador, amplificador y discriminador presentado en la Sección 2.1).

Respecto de la etapa de accionamiento la utilización de la etapa de accionamiento disponible presenta algunos inconvenientes, ya que los transductores electromecánicos descriptos tienen limitaciones de operación con esta técnica.

En la Figura 23 se presenta un diagrama en bloques simplificado del instrumento, en el cual puede observarse que el principio de operación es similar a la técnica de aceleración 
constante, con modificaciones en la unidad de procesamiento: el generador de referencia de velocidad debe proveer una señal de velocidad constante y el sistema contador debe medir tasas de arribo.

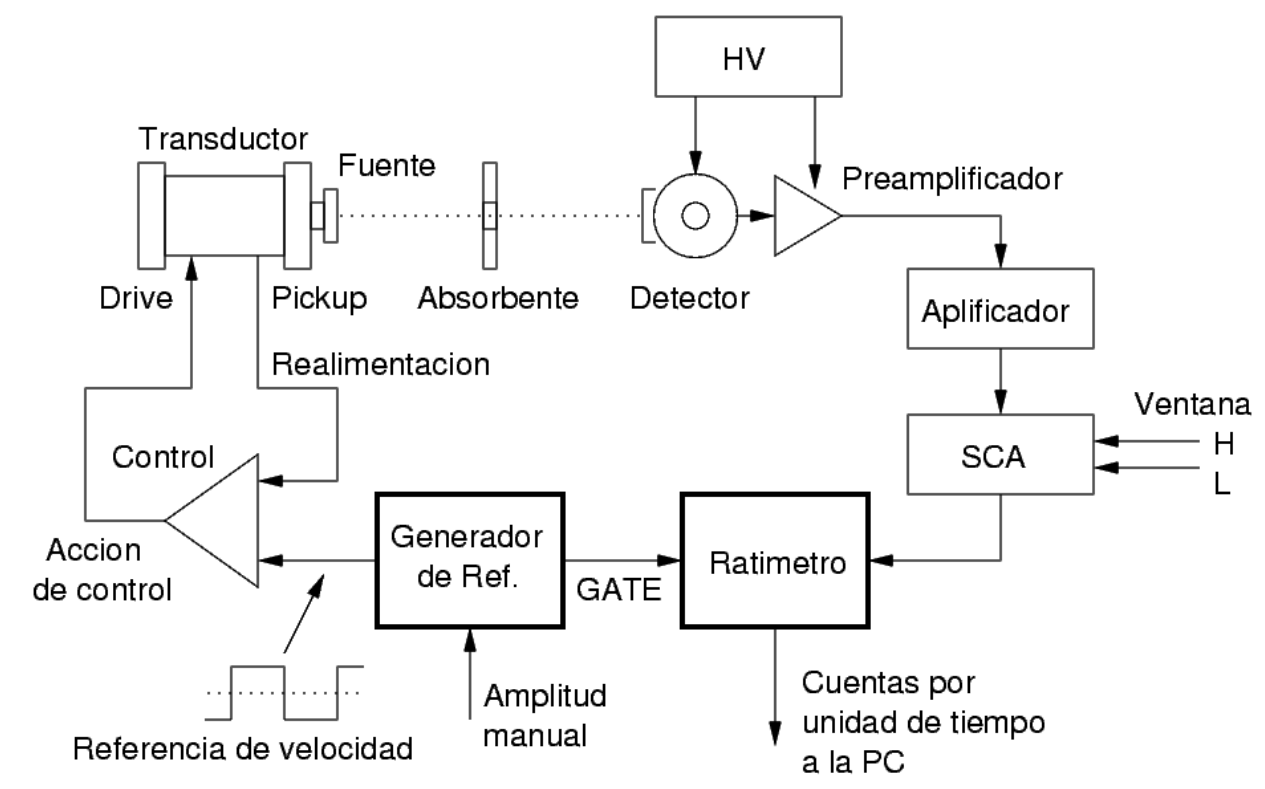

Figura 23: Diagrama en bloques de un espectrómetro Mössbauer de velocidad constante.

\subsubsection{El generador de referencia}

La opción más simple para este tipo de experimento es utilizar una referencia de velocidad cuadrada con valor medio nulo como se muestra en la Figura 23 . La parte superior de la onda cuadrada es equivalente al movimiento a velocidad constante de la fuente, y la parte inferior es el regreso del pickup, también a velocidad constante, para comenzar un nuevo recorrido a la velocidad de interés.

Una forma de aumentar el tiempo neto de medida es utilizar una referencia de la forma indicada en la Figura 24, donde el regreso se realiza a la máxima velocidad posible, teniendo en cuenta las limitaciones del transductor y del control de velocidad. Si la velocidad de retorno pudiese hacerse lo suficientemente grande, el tiempo de retorno sería despreciable frente al tiempo neto de medida [Lipkin 1964] [Chase 1968], con lo cual no sería necesaria la inhibición del contador. Para los transductores electromecánicos esta técnica no es eficiente, ya que no solo presentan limitaciones mecánicas sino que además los arrollamientos tienen un límite de corriente dado por el diámetro del alambre utilizado en su construcción. Por lo tanto el tiempo de retorno puede no ser despreciable.

Por lo tanto, la referencia debe estar sincronizada con una segunda señal lógica de inhibición del contador (GATE), la cual debe estar activa durante el tiempo de regreso y el tiempo de establecimiento de la velocidad deseada, tal como se muestra en la Figura 24 [Seberini 1988] [Veiga 1999]. 


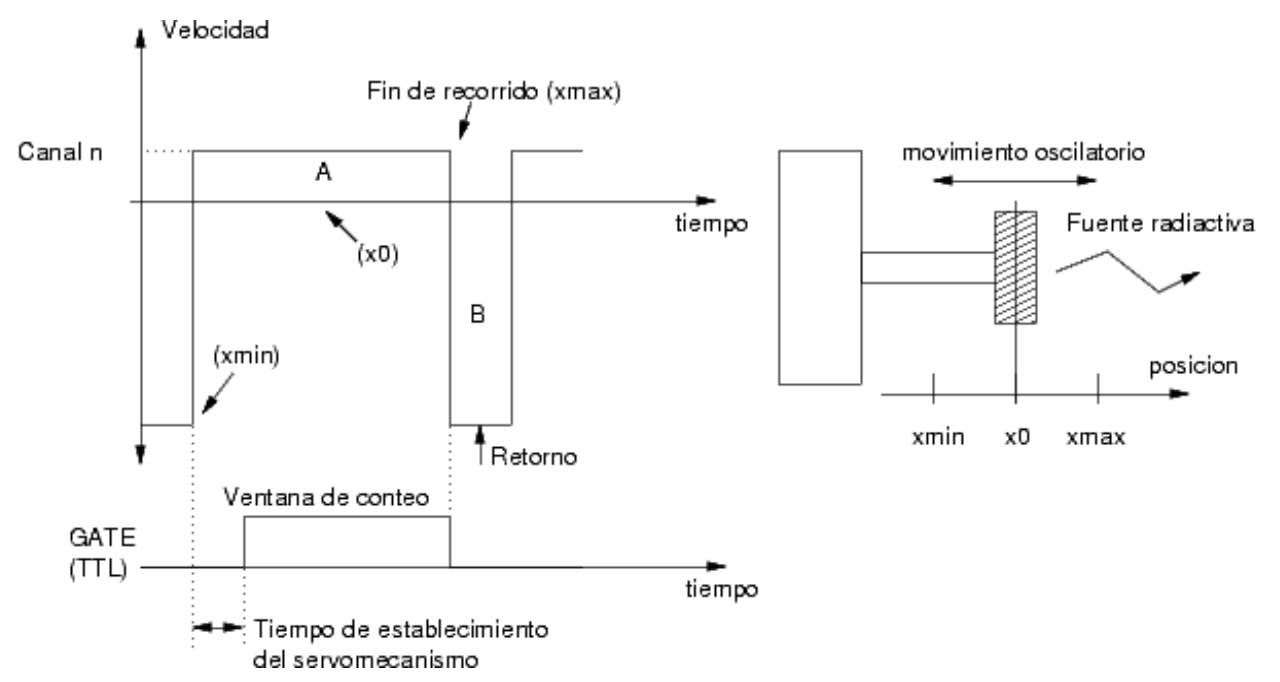

Figura 24: Referencia de velocidad para el experimento de velocidad constante. Las áreas A y $B$ deben ser iguales para mantener la fuente centrada en $x_{0}$.

La presencia de un alto contenido armónico en esta forma de onda, en contraste con la referencia triangular de aceleración constante, complica el control de la velocidad. Dicho contenido armónico aumenta cuanto mayor es la relación del tiempo de medida versus el tiempo de regreso (retorno a mayor velocidad para aumentar la eficiencia del instrumento). La presencia de una "meseta" en la referencia de velocidad produce un conflicto con la imposibilidad de acoplar el controlador continua. Por lo tanto la sintonización del control de velocidad es un punto crítico de este tipo de experimentos. Dado que los módulos de control de velocidad disponibles están en general optimizados para utilizar referencias triangulares, es difícil obtener una única sintonización óptima para un rango amplio de velocidades, debiéndose reajustar el control cada vez que se modifica la velocidad de interés. La utilización de una referencia trapezoidal como la de la Figura 25 reduce este problema [Veiga 1999], aunque no lo elimina definitivamente.

Para la generación de la referencia de velocidad y del GATE puede utilizarse un generador de funciones arbitrarias programable con dos salidas, o bien dos generadores sincronizados. Existen generadores comerciales, como es el caso del modelo DFG-1000 y 1200 de WissEl que agregan esta forma de onda a las tradicionales.

En nuestros laboratorios se utiliza un generador diseñado especialmente para el experimento [Veiga 2002], que trabaja con una eficiencia programable. Las formas de onda se almacenan digitalmente en un microcontrolador. Este dispositivo se encarga también de la generación precisa de la base de tiempo, de la generación de la señal de GATE y del manejo de un conversor D/A de 12 bits de precisión. La frecuencia de operación y el GATE son programables por hardware, con el objeto de sintonizar correctamente la adquisición de datos con la respuesta del transductor. Para su construcción se utilizaron amplificadores de bajo offset y bajo drift a fin de minimizar el esfuerzo del control de velocidad para su supresión. El cambio de amplitud (selección de la energía) se realiza manualmente a través de un potenciómetro. 


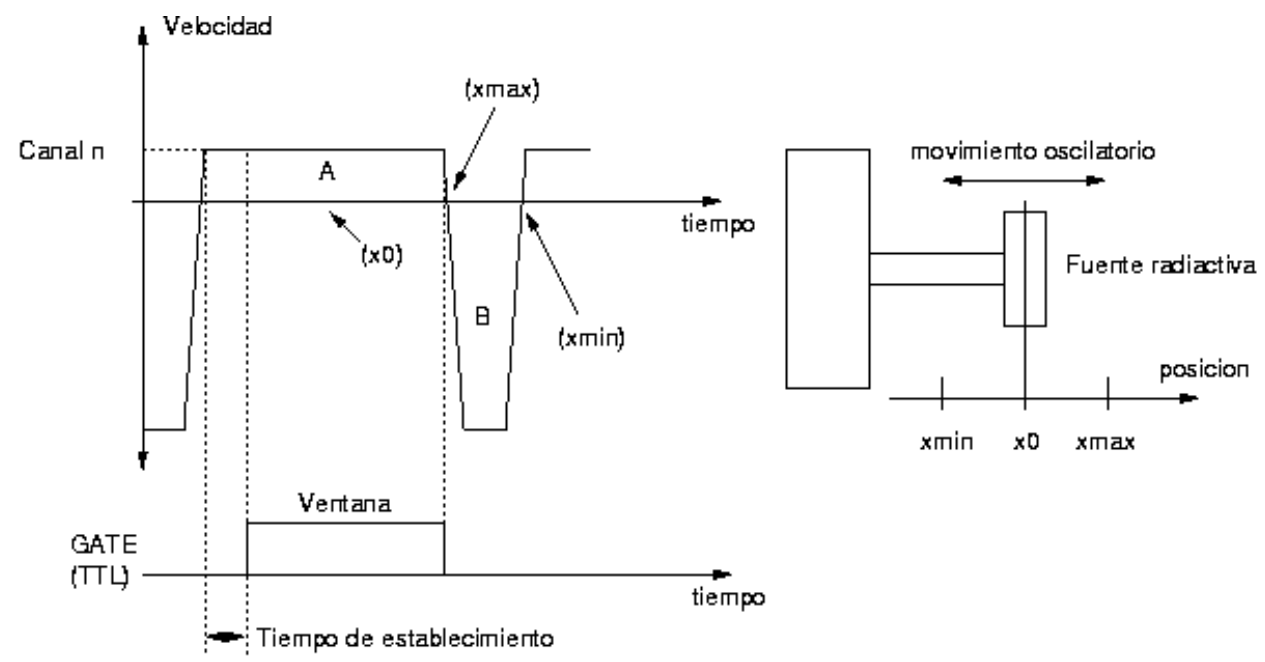

Figura 25: Referencia de velocidad trapezoidal para experiencias de velocidad constante.

\subsubsection{El ratímetro}

El objetivo de esta experiencia es medir la transmisión del material en estudio en función de un parámetro externo que varía con el tiempo, por lo tanto el contador debe ser del tipo ratímetro (medición de eventos en un determinado período de tiempo) y debe estar sincronizado con la evolución del parámetro externo. A fin de que sea posible automatizar la medida, el ratímetro debe disponer de algún tipo de interfaz a PC. Como se mencionó anteriormente, durante el establecimiento de la velocidad constante y durante el regreso del transductor, el conteo de eventos debe ser inhibido (GATE), facilidad debe estar disponible en el ratímetro.

Una diferencia importante respecto de la técnica de aceleración constante es en la magnitud del intervalo de conteo utilizado. En la técnica de aceleración constante se acumulan eventos en 256 canales diferentes o más, que son barridos una vez por cada ciclo de accionamiento del motor. En la técnica de velocidad constante los eventos se acumulan en un único elemento contador. Si se utiliza la técnica de aceleración constante con 256 canales y frecuencia de operación del transductor de $10 \mathrm{~Hz}$, resulta que cada $0.39 \mathrm{~ms}$ debe producirse un cambio de canal. En la técnica de velocidad constante el GATE inhibe sólo una vez por período del transductor. Si se opera a $10 \mathrm{~Hz}$ con eficiencia 0.75 , resulta un intervalo de conteo de $75 \mathrm{~ms}$. Existe, por lo tanto, una gran diferencia entre los intervalos de conteo de las distintas técnicas. Por ejemplo, si se utiliza una tasa de 10000 cuentas/s, en aceleración constante se obtienen 3 o 4 cuentas por canal y por intervalo de conteo. En cambio, a velocidad constante serán 750 . Si pierdo una cuenta sistemáticamente en un cambio de intervalo, en el primer caso el error puede llegar al $33 \%$, mientras que a velocidad constante sólo será de un $0.13 \%$. En este aspecto la técnica de velocidad constante es menos exigente que la de aceleración constante. De todos modos, la lógica rápida estándar (FAST TTL) satisface los requerimientos para ambas técnicas, por lo que la lógica digital del instrumento no es un punto crítico de diseño. Como se mostrará más adelante, el punto crítico en la performance de la etapa de detección es la conformación analógica del pulso, la cual es realizada por el amplificador. 
En nuestros laboratorios se utiliza un equipo con interfaz a PC basado en un microcontrolador, el cual fue diseñado especialmente para este tipo de aplicaciones [Veiga 2002]. Este permite la selección del tiempo de acumulación entre valores que pueden ir desde los $25 \mathrm{~ms}$ hasta varios minutos netos (sólo es acumulado el tiempo en que el GATE está alto), con una resolución de 600 ns. Transcurrido el período de tiempo neto seleccionado, el valor contado se vuelca a la PC a través de una interfaz digital. El contador tiene un tiempo muerto de 1.2 us, con un prescaler $\mathrm{x} 2 \mathrm{o}$ x4, lo cual permite utilizar directamente los pulsos de la salida lenta del discriminador. La utilización de un prescaler es posible en este caso ya que la ventana de conteo es mucho mayor que en las experiencias de aceleración constante.

\subsubsection{Aplicaciones específicas}

Un ejemplo de aplicación de este instrumento es la determinación de la temperatura crítica (Tc) de un material [Mendoza Zélis et al 2002]. Esta es la temperatura a la cual desaparecen las propiedades magnéticas del mismo, llamada Temperatura de Curie para los materiales ferromagnéticos y Temperatura de Neel para los antiferromagnéticos. Desde el punto de vista del espectro Mössbauer, el material en estudio evoluciona, al aumentar la temperatura del mismo, desde un sextete a temperatura ambiente hasta un singlete más allá de Tc (desaparece el desdoblamiento magnético hiperfino).

En la Figura 26 se muestra la evolución del espectro Mössbauer del antiferromagneto $\mathrm{FeSn}_{2}$ con el aumento de la temperatura. La temperatura crítica de este material es de de $379{ }^{\circ} \mathrm{K}$. Puede observarse que midiendo la transmisión solamente a la velocidad indicada por la línea vertical $a$ puede obtenerse el gráfico de la Figura 27, a partir del cual puede determinarse Tc con precisión. Obsérvese que la línea de interés no se encuentra centrada a velocidad nula, debido al corrimiento isomérico.

Este tipo de experiencias ha dado origen a la creación del Laboratorio de Barridos Térmicos Mössbauer del Departamento de Física de la UNLP, en el cual los instrumentos descriptos se encuentra actualmente operativo.

Otro ejemplo en el cual esta técnica encuentra aplicación es en el estudio del comportamiento de los materiales ante la presencia de un campo magnético externo. Este es un experimento novedoso que se desarrollado en conjunto con el Laboratorio Mössbauer, durante el transcurso de esta tesis [Pasquevich et al 2006]. Los detalles de la implementación se presentan en el Apéndice B. Se trata básicamente de mantener la absorción resonante en una energía de interés, mientras se aplica un campo magnético variable a la muestra en estudio. Las variaciones de dicho campo se controlan, utilizando instrumental diseñado específicamente, utilizando como referencia la salida triangular de una placa Nucleus MCSII. El conteo es registrado en el multiescalímetro. Se obtiene así, en forma automática, un espectro de absorción para una línea en función del campo magnético. Esta técnica ha demostrado ser de utilidad para el estudio de histéresis magnética de diferentes compuestos de hierro. 


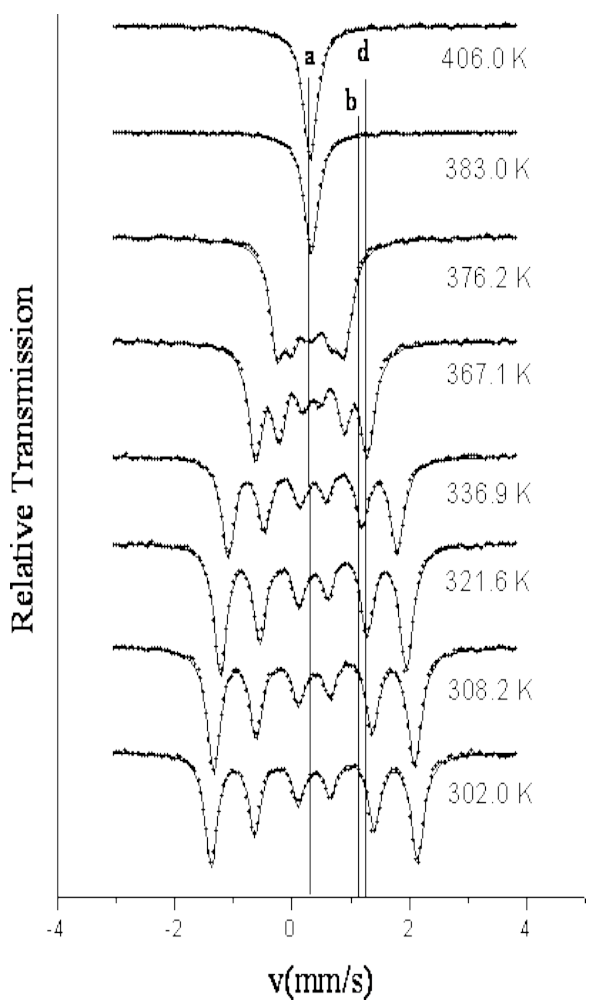

Figura 26: Espectro del antiferromagneto FeSn2 medido a diferentes temperaturas. Las líneas verticales indican tres velocidades de interés [Mendoza Zélis et al 2002].

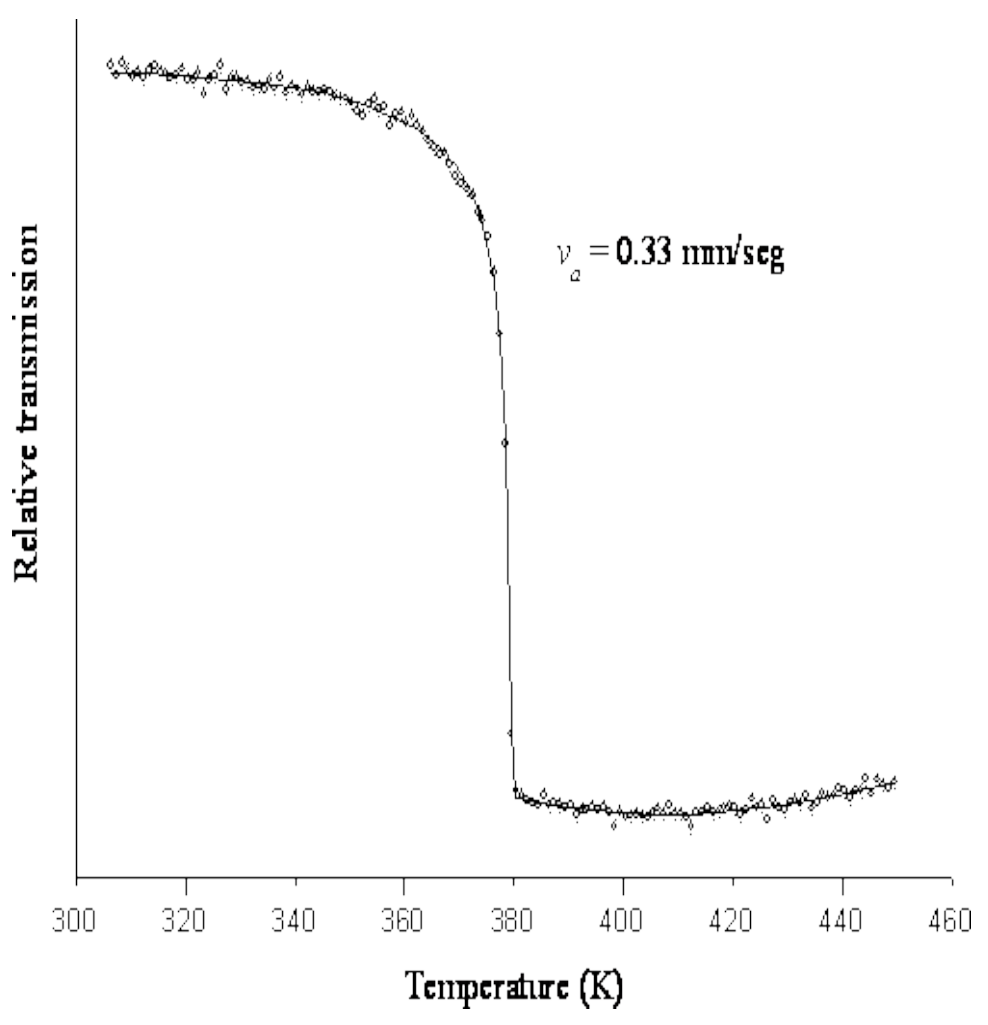

Figura 27: Barrido de temperatura correspondiente a la velocidad a de la Figura $26(0.33 \mathrm{~mm} / \mathrm{s})$ realizado con el instrumento de velocidad constante [Mendoza Zélis et al 2002].

\subsubsection{Puntos críticos y limitaciones de la instrumentación a velocidad constante}

Un aspecto crítico de esta configuración es la compensación del control de velocidad tal que opere con errores menores que $0.02 \mathrm{~mm} / \mathrm{s}$ con referencias del tipo de la Figura 24 o la Figura 25. Los transductores comerciales se encuentran optimizados para funcionar con referencias triangulares y sólo algunos pocos presentan buena respuesta a cuadradas simétricas. La imposibilidad de acoplar en continua dicho dispositivo (debido a la presencia inevitable de offset en la referencia) y el alto contenido armónico de la onda trapezoidal, hacen que deba ponerse especial cuidado en este punto. Pero con una sintonización cuidadosa y sistemática pueden obtenerse resultados satisfactorios. El barrido automático de velocidades se complica aún más ya que debe encontrarse un buena sintonización para varias amplitudes de la referencia manteniendo acotado el error absoluto.

De la calidad de la señal de GATE del contador depende la calidad de la medida, por lo tanto la inhibición debe ser precisa en el tiempo. Debe notarse que la ventana de conteo aumenta notablemente en esta experiencia respecto de la de aceleración constante. Esta condición relaja las restricciones de la electrónica asociada. 
Otro aspecto a tener en cuenta es que la ganancia del detector varía con la temperatura, desplazando el pico de $14.4 \mathrm{KeV}$ de la ventana seleccionada, disminuyendo así la tasa de conteo. En las experiencias de aceleración constante estas variaciones de ganancia se distribuyen equitativamente entre todos los canales, sin producir una distorsión del espectro. Esto se debe a que todos los canales son barridos varias veces por segundo y las variaciones de temperatura tienen constantes de tiempo mucho más largas. En cambio, para experiencias de velocidad constante, en las cuales sólo se observa una única velocidad, un cambio de temperatura produce una disminución de la tasa de conteo que no puede diferenciarse de la disminución producida por el efecto buscado. Para salvar este inconveniente debe mantenerse constante la temperatura del detector, lo cual es bastante complicado cuando se está calentando la muestra (que está muy próxima al detector), como es el caso de los barridos de temperatura.

Debe aclararse que la técnica de velocidad constante no es de aplicación tan frecuente como la de aceleración constante, principalmente por las complicaciones presentadas hasta aquí. En general, aprovechando la disponibilidad de las etapas de detección y accionamiento, suele utilizarse en forma rudimentaria como una técnica auxiliar en los laboratorios Mössbauer. Pero a menudo la dificultad en la puesta en marcha y la necesidad de una reconfiguración del instrumental desalientan su utilización. En este punto se basa la propuesta de un nuevo instrumento que presenta a continuación.

\subsection{Propuesta para un nuevo espectrómetro de velocidad programable}

\subsubsection{Motivación}

Considerando lo expuesto en las secciones anteriores, resulta deseable disponer de un único instrumento con la funcionalidad de los espectrómetros de aceleración y de velocidad constante. Pero además sería de gran utilidad poder relevar en forma automática el comportamiento de la muestra para varias energías, no necesariamente contiguas en el espectro, frente a una variación de parámetros externos. Para ello es necesario observar más de una línea, o una determinada región, pero no necesariamente el espectro completo como se hace con la técnica de aceleración constante. Con el instrumento de velocidad constante puede realizarse la observación, pero debe repetirse el barrido del parámetro externo para cada velocidad de interés, lo cual, además de ser engorroso, no siempre es posible (por ejemplo en el caso de estar en presencia de transformaciones irreversibles). El equipamiento de velocidad constante disponible actualmente tiene serias limitaciones para este tipo de experiencias, ya que no permite cambiar la referencia de velocidad automáticamente, y aún si fuera posible sería muy difícil hacerlo manteniendo la sintonía óptima del control de velocidad. Esta condición restringe considerablemente las posibilidades de automatización.

En términos generales, se desea liberar al espectrómetro Mössbauer de algunas de sus limitaciones importantes, como son que todos los espectros obtenidos son simétricos, centrados en velocidad nula y con una distribución uniforme en los canales. 


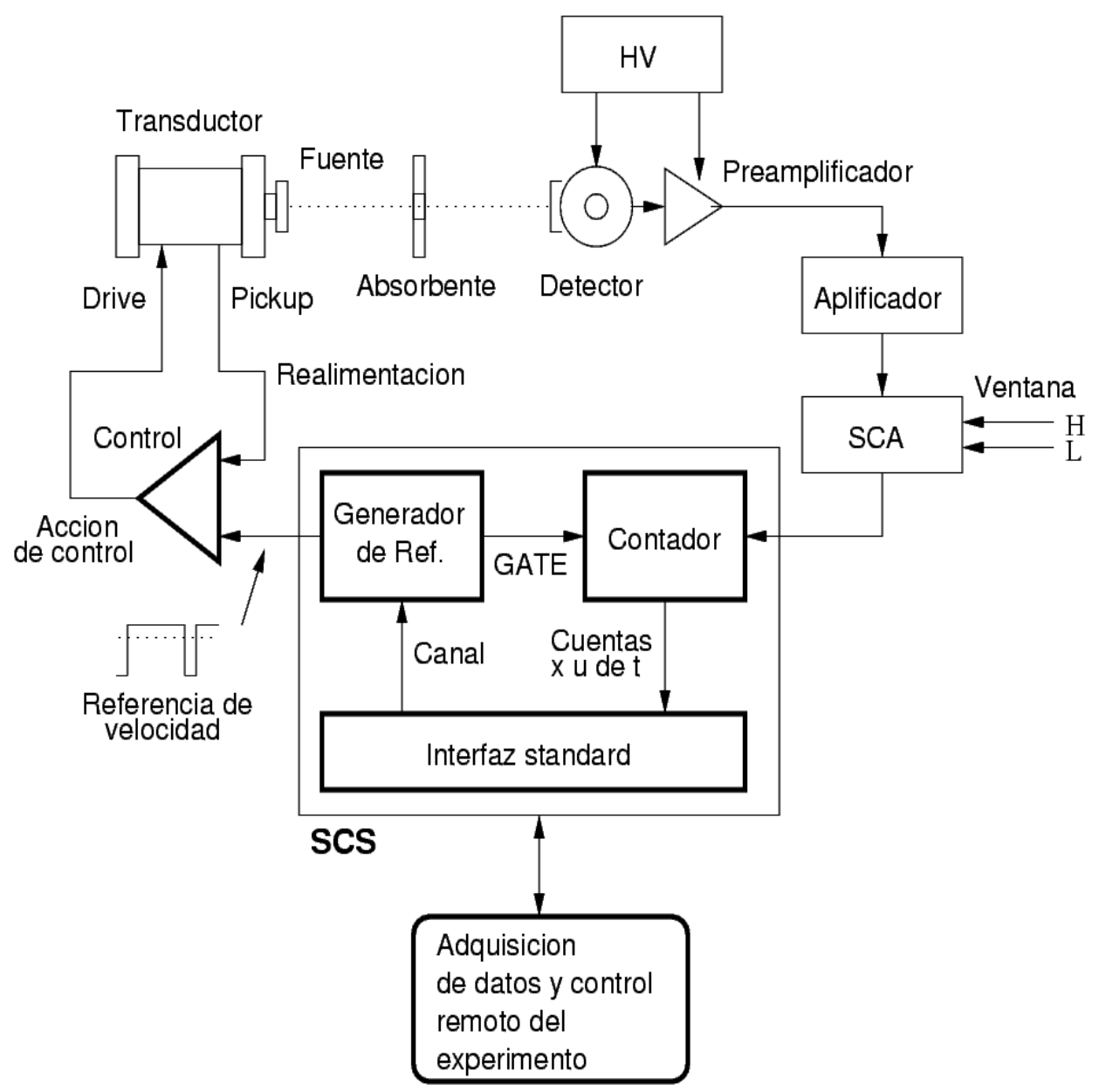

Figura 28: Diagrama en bloques propuesto para un espectrómetro Mössbauer de velocidad programable.

\subsubsection{Estructura general propuesta}

Se propone entonces el diseño e implementación de un nuevo espectrómetro de velocidad programable, basado en la técnica de velocidad constante. Se desea preservar, dentro de lo posible, la mayor cantidad de componentes tanto de la etapa de detección como de la etapa de accionamiento. El modo de funcionamiento propuesto para el nuevo instrumento es el siguiente: para cada canal del espectro deseado, se selecciona a través de una interfaz adecuada la velocidad requerida, proporcionando además el tiempo deseado de acumulación de cuentas; el equipo calcula la tasa utilizando la técnica de velocidad constante y luego de transcurrido el tiempo seleccionado el equipo retorna el número de cuentas acumuladas, quedando listo para recibir un nuevo pedido (en la misma velocidad o en una diferente). De esta manera manejando la interfaz se puede reconstruir la sección del espectro que sea de interés, procesando los canales de a uno. Esta técnica no utiliza un multicanal, sino que lo reemplaza por único canal programable con una interfaz adecuada.

El diseño del sistema propuesto se realizará sobre la base del diagrama de la Figura 28, buscando integrar los bloques indicados con línea gruesa en un dispositivo al que denominaremos escalímetro de velocidad programable (SCS, Single Channel Scaler). Éste 
será implementado respetando el estándar NIM, e incluirá una interfaz de comunicación remota para la automatización de medidas prolongadas.

\subsubsection{Consideraciones preliminares}

La etapa de detección debe ser optimizada para adecuarla a la nueva técnica de medición. Este aspecto es importante pues, inicialmente, el rendimiento de este instrumento será menor que el de aceleración constante, dado que la referencia de velocidad constante requiere que se inhiba el conteo durante el regreso, reduciendo el tiempo neto de medida. Esto incrementa la importancia de la optimización de la etapa de detección, por lo que se requerirá la minimización de tiempos muertos en los componentes analógicos (detector, preamplificador y amplificador), como así también el estudio de la técnica más adecuada para la discriminación de eventos.

La etapa de accionamiento debe ser perfeccionada para su operación a velocidad constante, preservando la mayor parte posible del equipamiento disponible. Debe realizarse un estudio sistemático de las formas de onda de referencia más adecuadas, y analizarse la posible utilización de algún tipo de realimentación de posición. Este conjunto de modificaciones debe habilitar la modificación automática de la velocidad en estudio.

La etapa de procesamiento del experimento debe ser replanteada por completo. El diseño estará basado en microcontroladores de última generación y técnicas de tiempo real.

Respecto de las energías en estudio, es necesario que todo el rango de velocidades entre -10 y $10 \mathrm{~mm} / \mathrm{s}$ pueda ser recorrido selectivamente, canal por canal, pero no necesariamente en forma continua. Se requieren al menos 256 canales, siendo deseables 512 y si fuera posible 1024. La resolución debe mantenerse al menos en $0.02 \mathrm{~mm} / \mathrm{s}$, a fin de producir espectros de calidad aceptable.

A modo de demostración de su buen funcionamiento, el equipo debe ser capaz de relevar en forma automática un espectro completo de una lámina de hierro de referencia, produciendo un ancho de línea equivalente al obtenido con los instrumentos de aceleración constante disponibles o mejor, con un rendimiento no inferior al 75\%. Debe ponerse especial énfasis en la linealidad del sistema, por ser éste un parámetro crítico que afecta sensiblemente la calidad de los resultados.

El sistema debe completarse con un programa de aplicación que permita observar el desarrollo del experimento, acceso remoto a las variables y los datos (incluso mientras se desarrolla la experiencia) y registro de los datos en un formato normalizado que permita el procesamiento posterior de la información. En este punto se aplicarán las herramientas de software y las técnicas de programación presentadas en la Tesis de Magister previa [Veiga 1999].

\subsubsection{Posibles aplicaciones: observación dinámica}

Además de la funcionalidad tradicional, el nuevo espectrómetro permitirá introducir las herramientas necesarias para una observación dinámica de los espectros de absorción resonante. Tanto el instrumental de laboratorio como la metodología de trabajo deben 
diseñarse con el objetivo de observar en forma detallada evoluciones temporales a través de la técnica Mössbauer, característica propia de los experimentos a velocidad constante. Para ello el instrumento debe poseer algunas características fundamentales, como el registro de datos detallado referido a una base de tiempo confiable, la posibilidad de re-configuración automática de los parámetros del experimento, la capacidad de automatización, y otras características propias de los experimentos modernos.

Una aplicación de este nuevo instrumento puede ser la observación simultánea de varias velocidades de interés mientras se modifica un parámetro externo. Tal es el caso de las velocidades representadas en la Figura 26 por las líneas verticales $b, c$ y $d$ para el $\mathrm{FeSn}_{2}$. En el caso de que esta experiencia se realizara con un instrumento de velocidad constante tradicional, debería efectuarse varias veces el ciclado térmico de la muestra (una vez para cada velocidad en estudio) lo cual no siempre es posible. En la Figura 29 se muestra la transmisión en función de la temperatura para dichos puntos. Con el nuevo instrumento debe ser posible obtener las tres gráficas, junto con la de la Figura 27, durante el transcurso de un único barrido térmico.

El nuevo instrumento puede utilizarse también para realizar un seguimiento eficiente de un mínimo de transmisión ante variaciones de un parámetro externo. Utilizando un algoritmo de detección de mínimos adecuado pueden realizarse espectros de unos pocos canales (sólo los necesarios para cubrir la sección de la línea que permita detectar el mínimo de transmisión) que se desplazan al evolucionar el parámetro externo, consiguiéndose así el seguimiento de la línea con tiempos de medida mucho menores que los que serían necesarios en caso de utilizarse alguno de los espectrómetros tradicionales. Esta técnica permitirá estudiar en forma dinámica la dependencia de las propiedades de los materiales con las variaciones de temperatura, pudiéndose detectar efectos de histéresis térmica u otros fenómenos similares.

Esta técnica de medición canal por canal se puede utilizar además para obtener espectros completos, equivalentes a los obtenidos con un instrumento tradicional de aceleración constante, necesarios en el momento de la calibración del instrumento. También pueden obtenerse espectros parciales no centrados en velocidad nula, lo cual no es posible a aceleración constante, como así también pueden medirse velocidades sólo positivas o sólo negativas.

También la técnica de velocidad constante tradicional puede mejorarse con el nuevo instrumento. Las variaciones en la ganancia del detector mencionadas en la Sección 2.4.5 pueden medirse si además del canal de interés se monitorea periódicamente el fondo del espectro con un canal adicional.

Resumiendo, el nuevo equipamiento permitirá eliminar algunas de las limitaciones que presentan las técnicas tradicionales de aceleración y velocidad constante en aplicaciones específicas, preservando la funcionalidad de ambas. En la actualidad, modificar un espectrómetro de aceleración constante para que opere a velocidad constante requiere de una re-configuración completa del instrumento y de una nueva calibración, lo cual hace engorroso el procedimiento. Esto ha llevado a algunos laboratorios a disponer de dos espectrómetros completos en funcionamiento.

En los próximos capítulos se presentarán las contribuciones realizadas durante el transcurso de esta tesis con el objetivo de solucionar este problema. En el Capítulo 3 se presentan 


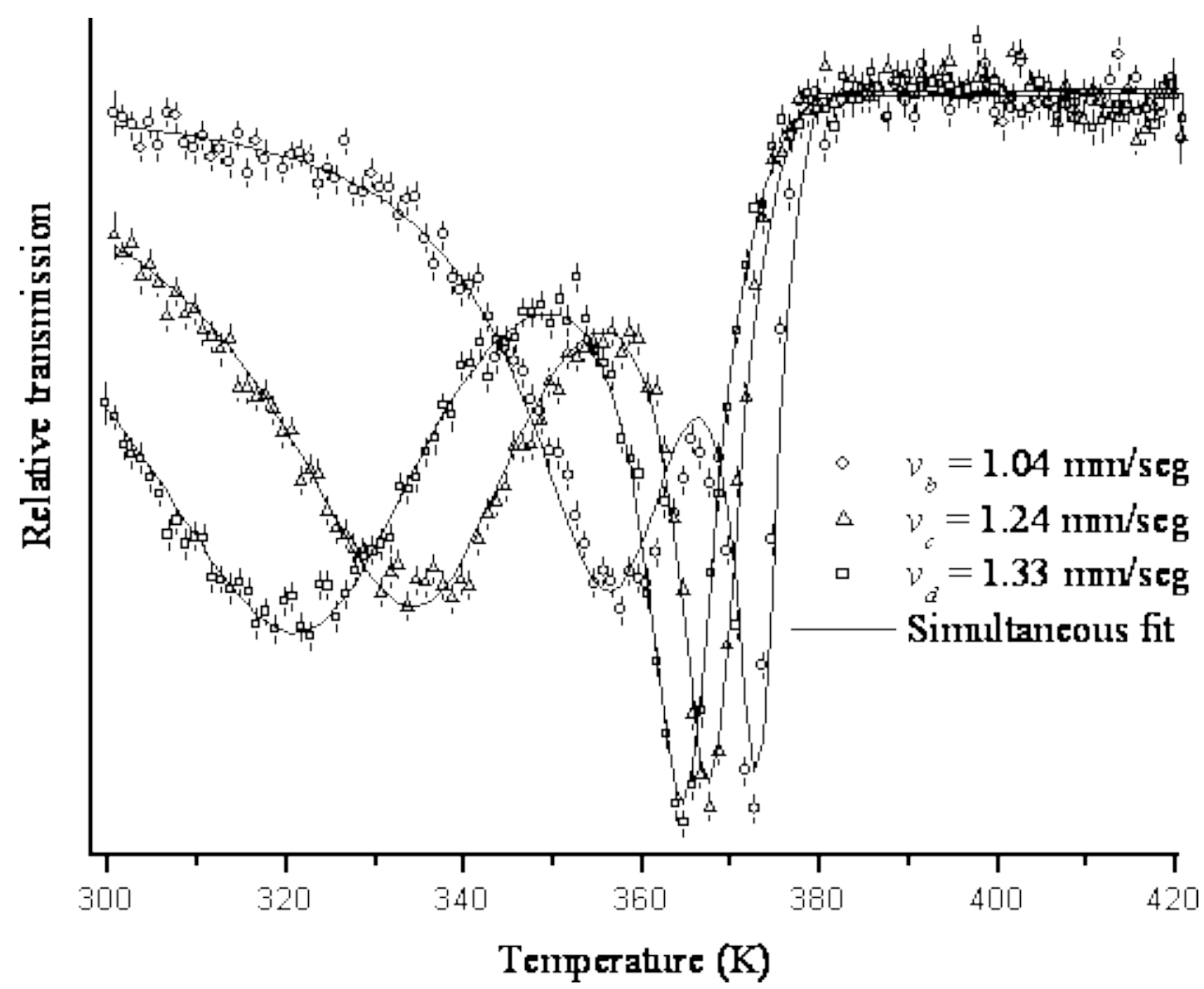

Figura 29: Barridos térmicos realizados a tres velocidades diferentes (b, c y d de la Figura 26) con un instrumento de velocidad constante [Mendoza Zélis et al 2002]. El ciclado térmico debió repetirse para cada velocidad de interés.

algunos aportes originales tendientes a mejorar la eficiencia de la etapa de detección; en el Capítulo 4 se optimiza el funcionamiento de los transductores para su operación a velocidad constante; y en el Capítulo 5 se diseña una nueva etapa de procesamiento, adecuada a las necesidades de la nueva disposición experimental. 


\section{Notas acerca del nuevo modo de funcionamiento}

El espectrómetro de velocidad programable propuesto tiene algunas particularidades que lo diferencian de los espectrómetros que utilizan las técnicas de aceleración o velocidad constante. Para explicarlas puede hacerse una analogía con la captura de imágenes fotográficas y de video.

En principio, puede pensarse en las opciones que existen para registrar el movimiento de un objeto. Puede utilizarse una cámara fotográfica de alta definición capaz de tomar unas pocas imágenes por segundo, o puede registrarse el movimiento utilizando una filmadora que, si bien captura un área más pequeña, en un segundo puede tomar 25 imágenes o más. Para describir la trayectoria del objeto en movimiento puede ser preferible la segunda opción, siempre que el objeto esté la mayor parte del tiempo comprendido dentro de la pequeña imagen registrada. Si se piensa en el espectro Mössbauer como en una imagen unidimensional, la cámara fotográfica de alta definición es equivalente al espectrómetro de aceleración constante, mientras que la cámara de video funciona como el de velocidad programable (la técnica de velocidad constante sería equivalente a capturar un único pixel).

Continuando con la analogía, debe notarse que en las experiencias dinámicas siempre existirá un compromiso entre el tiempo de exposición $t_{e}$ y la cantidad de imágenes registradas por segundo $c_{p \mathrm{~s}}$ tal que $c_{p s M A X}=1 / t_{e}$. Un mayor $t_{e}$ implica mayor sensibilidad, aunque se obtendrá un menor número de imágenes por segundo, lo que deteriora la sensación de movimiento. En este sentido, el espectrómetro de velocidad programable permite disminuir la cantidad de puntos de la imagen para disminuir $t_{e}$, manteniendo la sensibilidad. Eso no es posible con la técnica de aceleración constante. El mecanismo es equivalente al zoom digital del que disponen las cámaras de video actuales, aunque no es posible encontrar un equivalente óptico. Sin embargo, en este punto existe una diferencia importante con la captura de video. La disminución en el número de puntos no se realiza aumentando el tamaño del pixel, como en el video de baja calidad (eso es lo que haría la técnica de aceleración constante al registrar en menos canales). Se hace utilizando puntos igualmente pequeños, incluso más pequeños aún, que se colocan a la distancia deseada. El resto de la imagen se obtiene por interpolación, lo que se ve simplificado por tratarse de una imagen unidimensional. En la Sección 6.3 se presentará un experimento que utiliza esta funcionalidad para realizar espectros dinámicos con información concentrada en la zona de interés.

Finalmente, la nueva técnica dispone también de la capacidad equivalente al zoom óptico. Pueden concentrarse todos los canales disponibles en una zona del espectro que se desea relevar con mayor detalle, en forma estática. Un ejemplo de aplicación de esta capacidad se presentará en la Sección 6.4, donde se utiliza toda la resolución del espectrómetro para obtener la máxima información en una zona de especial interés del espectro. 


\section{Capítulo 3 \\ Optimización de la etapa de detección}

En esta tesis se propone una nueva metodología aplicable a espectroscopia Mössbauer, compuesta por una combinación de las técnicas existentes de velocidad y aceleración constante, a la que llamamos de velocidad programable.

Para la implementación de la nueva metodología es deseable utilizar la mayor parte de los componentes disponibles en un laboratorio de espectroscopia Mössbauer tradicional. Tal es el caso de la etapa de detección, tal como fue descripta en el capítulo anterior. Ésta puede ser utilizada en su totalidad ya que se trata de una etapa de selección de alturas de pulsos similar a la disponible en diversos experimentos de física nuclear.

Sin embargo, dado que con la nueva disposición se desean observar fenómenos dinámicos, la eficiencia en la detección de la resonancia cobra una importancia fundamental. En las experiencias estáticas el tiempo también es importante, pero una baja eficiencia repercute sólo en un aumento del tiempo total de medida y por lo tanto en un bajo rendimiento del instrumental de laboratorio, pero no ocasiona resultados incorrectos. Por el contrario, en experiencias dinámicas, si lo que se desea es observar un fenómeno que evoluciona en el tiempo, la frecuencia con que se observa el mismo es representativa de la calidad de la medida (frecuencia de muestreo). Incluso puede ser determinante para la factibilidad de algunas observaciones en las que el fenómeno evoluciona rápidamente.

A lo largo de este capítulo se presentarán algunos criterios de optimización de la etapa de detección, desarrollados durante el transcurso de esta tesis, orientados a aumentar la eficiencia del instrumento de velocidad programable. Debe destacarse que gran parte de los resultados y conclusiones obtenidas son de aplicación tanto en la nueva técnica como en experiencias Mössbauer tradicionales de aceleración constante o de velocidad constante.

En principio, este capítulo revela los puntos de la etapa de detección sensibles a optimización, 
presentando luego un nuevo método para la selección de la tasa de trabajo (distancia de la fuente) y del criterio de selección de eventos (ventana del discriminador). Como se mostrará, estos dos parámetros de trabajo son determinantes de la calidad de los resultados. A pesar de ello en los laboratorios suelen ajustarse en forma empírica, recurriendo a la experiencia de los operadores, sin un criterio formal de optimización.

El punto de partida para la optimización es una etapa de instrumentación nuclear clásica para detección de altura de pulsos. Tal como se detalló en el capítulo anterior, la etapa está compuesta por un detector del tipo contador proporcional, un preamplificador de carga, un amplificador conformador de pulsos y un discriminador diferencial (SCA) para la selección de eventos. El diagrama esquemático se repite en la Figura 30, incluyendo el elemento contador digital que se describió como perteneciente a la etapa de procesamiento. Este dispositivo será un multiescalímetro para la técnica de aceleración constante, un ratímetro para la técnica de velocidad constante, o la entrada de la nueva etapa de procesamiento para la técnica de velocidad programable. Éste se incluye en este capítulo ya que algunos de los aspectos analizados condicionan su selección o eventual diseño.

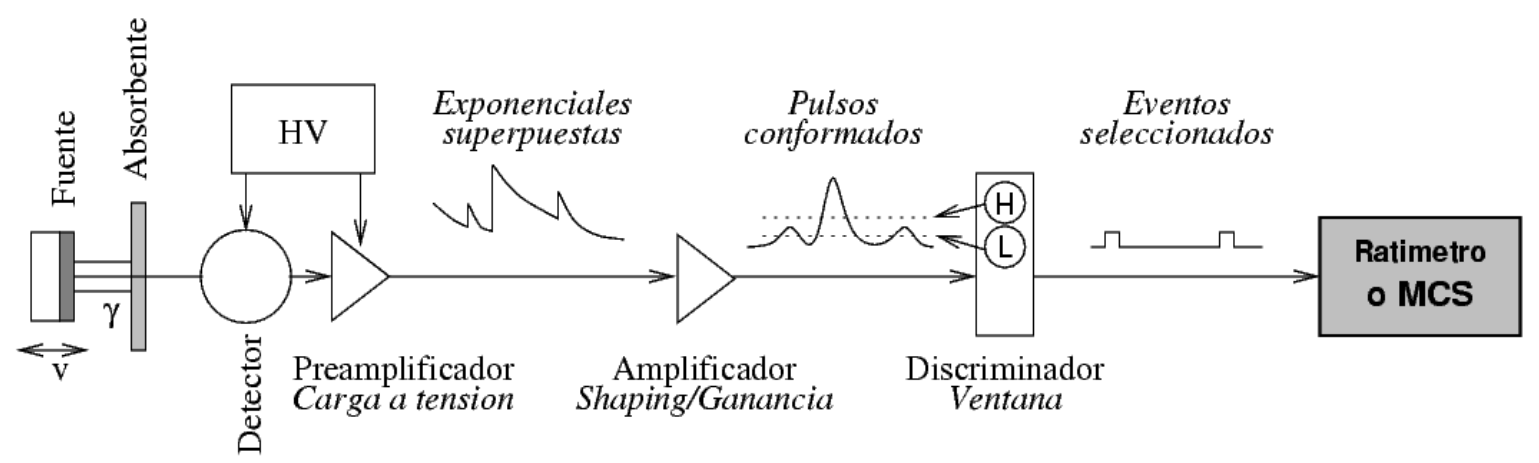

Figura 30: La etapa de detección, incluyendo el elemento contador.

En este capítulo se presentarán primero las definiciones de dos parámetros que pueden utilizarse para reflejar la calidad de las medidas, como son la relación señal a ruido del espectro y la eficiencia del instrumento. A continuación se presenta un estudio detallado de la técnica de selección de eventos con discriminador, obteniéndose algunos resultados que permiten mejorar la eficiencia. Se procede luego con un estudio de los tiempos muertos de la etapa de detección y su influencia sobre dichos parámetros de calidad y el tiempo de medida. Finalmente se presentan los requerimientos que imponen dichos criterios de optimización a los dispositivos digitales de la etapa (discriminador y contador) que se utilizan para completar el conteo de eventos. El capítulo se cierra con una propuesta de procedimiento de optimización de la etapa.

\subsection{Relación señal a ruido de un espectro}

La adquisición de un espectro Mössbauer es un proceso estadístico. El error será menor cuanto mayor sea el tiempo de medida. En general, es deseable mantener al mínimo el tiempo que insume la tarea, sobre todo si se desean estudiar fenómenos dinámicos. Por lo tanto, el tiempo de medida por canal es una variable importante a ajustar para el experimento. Los 
criterios de selección serán diferentes para cada caso particular de aplicación, pero puede asumirse que siempre estará presente el compromiso entre el tiempo y la calidad de la medida.

Para el estudio de fenómenos estáticos, los espectrómetros Mössbauer tradicionales tienen la particularidad de que el ruido estadístico del espectro puede reducirse tanto como se desee, a expensas del tiempo de adquisición. Esto hace que una optimización de la etapa de detección no sea crucial para el éxito del experimento. Sólo es necesario disponer del suficiente tiempo para llevarlo a cabo.

Para el estudio de fenómenos dinámicos, en cambio, el tiempo de medida puede ser determinante para la factibilidad del experimento. En este contexto es importante conseguir una optimización de la etapa de adquisición. Esto implica obtener la mayor tasa de eventos posible, a fin de aumentar rápidamente la estadística. Sin embargo, como se mostrará a continuación, son varios los factores que limitan este aumento.

Con el objetivo de clarificar este compromiso se presentará el concepto de relación señal a ruido del espectro, y se presentarán los factores que influyen sobre este parámetro.

Dada una tasa de $m$ eventos (fotones) por segundo, luego de transcurrido un cierto tiempo de adquisición se habrán acumulado $M$ cuentas por canal en el fondo, lejos de la línea, y $M_{a}$ cuentas, equivalentes a una tasa $m_{a}$, en la línea de absorción, como muestra la Figura 31.

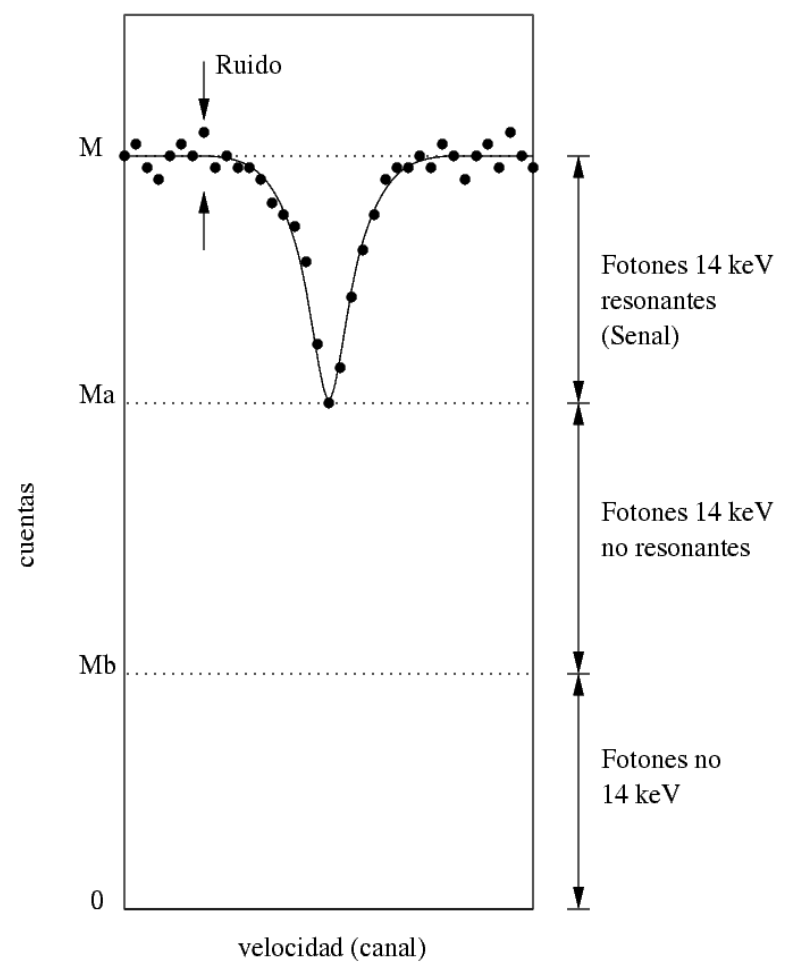

Figura 31: Relación señal a ruido en un espectro Mössbauer, dada por la relación entre la proporción de absorción de la línea resonante y el ruido estadístico. 
Los $M_{a}$ eventos que no resonaron se componen de $M_{b}$ eventos que no son de $14 \mathrm{KeV}$, más una fracción de los de $14 \mathrm{KeV}$ que no resuena. Esta última depende de la fracción LambMössbauer y del espesor del absorbente, como se mostró en el Capítulo 1. Resulta un efecto Mössbauer $f$ (medido) que estará dado por la expresión:

$$
f=\frac{M-M_{a}}{M}=1-\frac{M_{a}}{M}
$$

Para un determinado tiempo de medida $T$ se cumple que $M=m T$ y que $M_{a}=m_{a} T$. Expresando $f$ como relación de tasas se obtiene:

$$
f=\frac{m-m_{a}}{m}=1-\frac{m_{a}}{m}
$$

Por ejemplo una lámina de hierro natural de 12 um como las utilizadas para calibración, a temperatura ambiente tiene un efecto de aproximadamente el $20 \%$ en la sexta línea.

La emisión de la fuente radiactiva puede modelarse como un proceso aleatorio con una distribución exponencial de media M y desviación estándar $\sigma=\sqrt{M}$. El error estadístico relativo resulta $\sqrt{M} / M$. En estos términos, la relación señal a ruido puede definirse como:

$$
\frac{S}{N}=\frac{\text { efecto }}{\text { error estadístico }}=\frac{\frac{M-M a}{M}}{\frac{\sqrt{M}}{M}}=\frac{M-M a}{\sqrt{M}}
$$

Utilizando la ecuación (5) la relación puede expresarse como:

$$
\frac{S}{N}=\frac{f M}{\sqrt{M}}=f \sqrt{M}
$$

La relación señal a ruido puede utilizarse como un parámetro de calidad del espectro. Al aumentar el conteo, o ante la presencia de un mayor efecto, la medición será menos 'ruidosa' y el ajuste se realizará con mayor exactitud.

Consideremos ahora los efectos del tiempo de medida sobre la relación señal a ruido, utilizando dicha expresión. Si $m$ es la tasa media y $N_{c}$ el número de canales, la tasa por canal $m_{c}$, lejos de una línea de absorción, puede expresarse como:

$$
m_{c}=\frac{m}{N_{c}}
$$

Si se mide durante un tiempo $T$, en cada canal se acumularán $M$ cuentas, como muestra la 
Figura 31, cuyo valor estará dado por:

$$
M=m_{c} T=\frac{m}{N_{c}} T
$$

de donde puede obtenerse una expresión para $T$ :

$$
T=M \frac{N_{c}}{m}
$$

Utilizando $M$ de la expresión (8), el tiempo total de medida $T$ puede expresarse, entonces, como:

$$
T=\frac{(S / N)^{2}}{f^{2}} \frac{N_{c}}{m}
$$

Se observa que el tiempo total que insumirá la medida, necesario para obtener una relación señal a ruido determinada, depende de la tasa, del efecto y del número de canales en que se reparte la estadística.

Por ejemplo, para una tasa de $10000 \mathrm{c} / \mathrm{s}$, utilizando 256 canales, suponiendo que no se pierde ningún evento a causa de la etapa de procesamiento, y considerando un efecto del $20 \%$ $(f=0.2)$, si deseo una relación señal a ruido de $40 \mathrm{~dB}(S / N=100)$ resulta por ecuación (12) que necesito al menos 100 minutos de medición. Si deseo una relación señal a ruido de $60 \mathrm{~dB}$, resultará necesario medir al menos 7 días.

Como puede verse, el tiempo es crítico en este tipo de experimentos. El instrumento debe mantenerse estable durante períodos prolongados de tiempo, lo cual es complicado dada la sensibilidad de la etapa de detección respecto de la temperatura.

Por otro lado, debido a los prolongados tiempos de medición necesarios, es difícil, y hasta a veces imposible, realizar estudios de dinámicas rápidas por medio de la técnica de velocidad constante. Todos los cambios o barridos de parámetros deben ser muy lentos para permitir colectar la estadística en cada punto de interés, lo que puede condicionar la implementación de ciertos experimentos.

Respecto del espesor de la muestra, como se mencionó en el Capítulo 1, existe un espesor óptimo para cada material, el cual permite minimizar el tiempo necesario para obtener una determinada calidad del espectro. En este sentido, a partir de la definición presentada en ecuación (12), se enumeran a continuación los criterios generales que pueden aplicarse para minimizar el tiempo con el mismo criterio.

i. Aumentar el efecto medible $f$. Este depende de la fracción Lamb-Mössbauer propia del material, del espesor del absorbente y de la proporción de fotones con probabilidad de resonar sobre el total. En este último aspecto, una selección estricta de eventos (una ventana angosta) es importante. Sin embargo, existe aquí un compromiso con el siguiente punto. 
ii. Aumentar la tasa $m$. La tasa disminuye si se utiliza un criterio de selección muy estricto, lo cual presenta un compromiso con el punto anterior. Una forma de aumentar la tasa sin modificar la ventana es acercando la fuente, si la actividad de la misma es suficiente, siempre respetando los criterios de ángulo sólido. Por otro lado es importante disponer de una etapa de detección capaz de registrar la totalidad de los eventos, perdiendo la menor cantidad posible a causa del tiempo muerto

iii.Usar la menor cantidad posible de canales, tal que la estadística se reparta sólo en el área de interés. Para esto se introduce seguidamente el criterio de eficiencia del instrumento, el cual permitirá realizar más adelante comparaciones entre las diferentes técnicas de medición disponibles, incluida la nueva técnica propuesta.

Luego de dicha definición se procederá a cuantificar los efectos que tienen el tiempo muerto de la etapa de detección y el tamaño de la ventana sobre el tiempo de medida, mostrando los posibles criterios de optimización disponibles.

\subsection{Eficiencia de la etapa de detección}

Una definición de eficiencia, relativa a un instrumento, debe hacer referencia a la optimización que el mismo hace de los recursos. Debe ser una medida del grado en que la medición realizada alcanza sus objetivos, optimizando el uso de los recursos disponibles. Dicho de otra forma, un instrumento será mas eficiente cuando demuestre mayor capacidad para reducir al mínimo los recursos utilizados para alcanzar los objetivos.

Partiendo de una cierta disponibilidad de instrumentos en un laboratorio de espectroscopia, el recurso a optimizar es el tiempo de medida. En este marco, el parámetro eficiencia (en términos relativos) puede utilizarse, junto con la resolución y la linealidad, para caracterizar las bondades de un instrumento, sobre todo en el caso que se busca optimizar (operación a velocidad constante programable).

En algunos experimentos el concepto de eficiencia es intuitivo. Por ejemplo, dada la tarea de obtener un espectro Mössbauer completo de una muestra de hierro entre -10 y $+10 \mathrm{~mm} / \mathrm{s}$, el instrumento más eficiente será el que tarde menos tiempo en conseguir la relación señal a ruido deseada. Sin embargo, en términos más generales, para cualquier experimento será más eficiente el instrumento que tarde menos tiempo en obtener el parámetro físico buscado con el error requerido. El espectro en sí no es la información buscada, sino los parámetros que se extraen de él.

Entonces un nuevo instrumento será más eficiente que los convencionales si permite obtener el espectro mínimo necesario para calcular el parámetro de interés. Esto implica no perder estadística en canales redundantes de un espectro completo si no es necesario. Pero para que este razonamiento sea aplicable es necesario disponer de algo de información respecto del material en estudio. Por ejemplo, si se desea estudiar la dinámica de un material totalmente desconocido puede ser recomendable obtener previamente información respecto de su estructura en forma estática con la técnica de aceleración constante.

Es importante notar que la disminución del tiempo de medida proviene tanto de incrementar la tasa de conteo del experimento como de medir sólo en los sitios de interés. Ambos 
objetivos se persiguieron en el transcurso de esta tesis, el primero en este capítulo y el segundo en el próximo.

Entonces, cuando se enuncie que un instrumento es más eficiente que otro, será siempre respecto de un parámetro de interés. Ese mismo instrumento puede no ser eficiente para obtener un parámetro diferente. Por ejemplo, cuando se desee obtener un espectro centrado en velocidad nula para un determinado rango de velocidades, el instrumento más eficiente será siempre el espectrómetro de aceleración constante. La eficiencia es máxima pues todos los eventos son detectados y se acumulan en algún canal del espectro de interés. Sin embargo, en el caso en que sólo se busque obtener la posición de una determinada línea de absorción, puede ser más eficiente utilizar un espectrómetro de velocidad constante, aunque el tiempo neto de medida del mismo sea del $75 \%$ del tiempo real.

\subsection{Cálculo de la ventana óptima}

Un aspecto importante de la etapa de detección es el criterio utilizado para la selección de los eventos que producen absorción resonante. En la práctica se utiliza un discriminador diferencial (SCA), seleccionando por altura los pulsos a la salida del amplificador. Los pulsos con alturas correspondientes a un determinado rango (o ventana) serán los que tienen la energía exacta para producir absorción resonante. En la Sección 2.1.4 se mostraron las técnicas utilizadas para la calibración de dicha ventana, junto con sus complicaciones experimentales.

Pero aún suponiendo que la ventana puede calibrarse con precisión, resta determinar cuál es el tamaño de ventana que permite optimizar la relación señal a ruido, según fue definida en la ecuación (7). Este tema no ha sido muy tratado en la bibliografía y se desarrollará a continuación.

Evidentemente existe un compromiso entre las siguientes cuestiones: si se utiliza una ventana muy cerrada se consigue una buena selectividad de eventos (lo cual mejora la relación señal a ruido), pero se obtiene una baja tasa de eventos (lo cual la desmejora); si se utiliza una ventana muy abierta, la estadística mejora, pero a su vez aumentan los eventos no resonantes, desmejorando la relación señal a ruido. Entonces debe existir un tamaño óptimo para la ventana.

En lo que sigue de esta sección se encontrará una expresión que permite calcular el tamaño óptimo de la ventana, utilizando el modelo simplificado de la Figura 32. Este modelo supone que el espectro de energía a la salida del amplificador en las inmediaciones de la zona de interés es una gaussiana, con una altura $L$ en el valor más probable y una desviación estándar $\sigma$. Esta distribución está montada sobre la superposición de varios efectos no resonantes (las colas de las gaussianas correspondientes a los rayos $\mathrm{X}$ de mayor y menor energía, más un fondo de scattering, ruido, etc.). A esta combinación se la supondrá uniforme en energía y de valor $B$. Los parámetro $L, B$ y $\sigma$ pueden obtenerse con un multicanal conectado a la salida del amplificador. Para ello es necesario que la fuente se encuentre a la distancia de trabajo, con el absorbente en posición, y debe garantizarse una velocidad de la fuente tal que no se produzca absorción resonante ${ }^{3}$.

3 Este es un nuevo ejemplo de aplicación de la técnida de velocidad constante. 
El total de los fotones (área bajo la gaussiana) tiene dos componentes:

i. Fotones sin probabilidad de resonar, determinados por el área rectangular debajo de B.

ii. Fotones con probabilidad de resonar, determinados por el área total bajo la curva menos el área rectangular debajo de $\mathrm{B}$, correspondientes al nivel de energía de interés.

De estos últimos sólo producen absorción resonante una proporción $f_{i}$ que depende del coeficiente Lamb-Mössbauer y del espesor del absorbente. El efecto $f$ estará determinado por la proporción entre los eventos resonantes y el total de los mismos. Resulta así un espectro de absorción como el mostrado en la figura Figura 32.
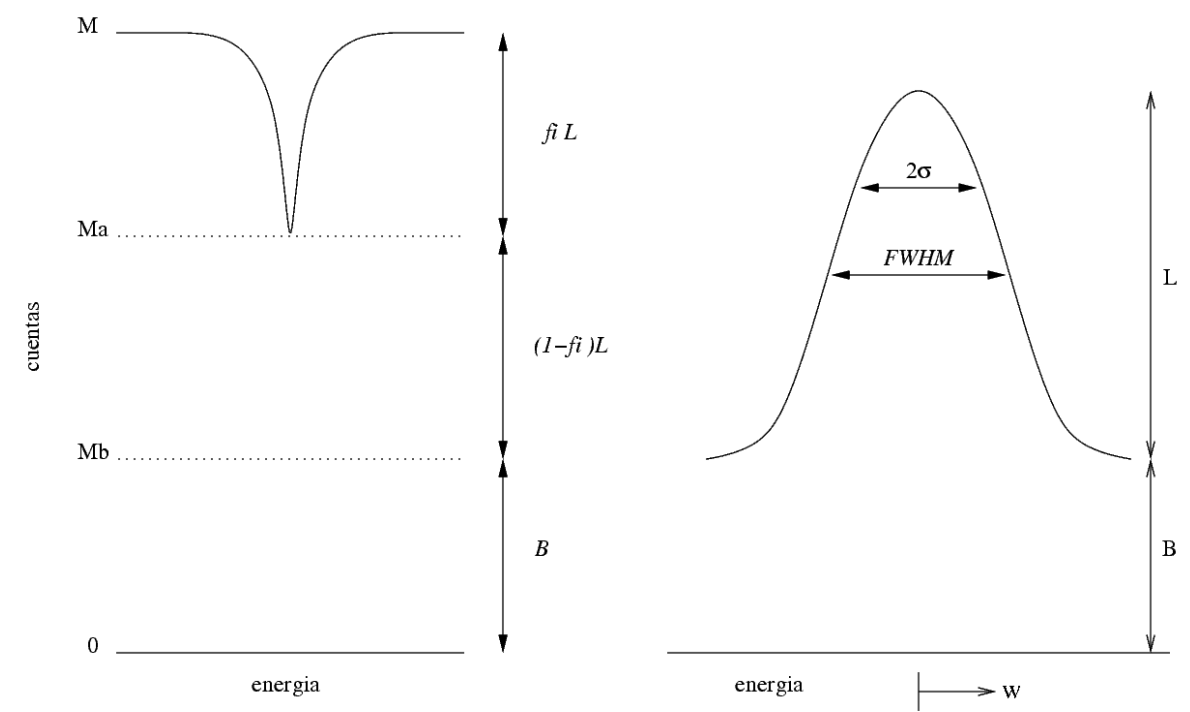

Figura 32: a) Sexta línea de absorción resonante del espectro del hierro natural. b) Aproximación del espectro de energía de la fuente de $\mathrm{Co}^{57}$ en las inmediaciones de $14 \mathrm{KeV}$.

Según la definición de la relación señal a ruido presentada en la ecuación (7), y definiendo la apertura de la ventana $w$ como muestra la Figura 32, puede encontrarse una expresión de la relación señal a ruido en función de dicha ventana, con $f_{i}$ y la forma del espectro $(\sigma, B$ y $L)$ como parámetros. Para ello se calcula la relación entre el número eventos que resuenan y el número total de eventos, entre el centro y la ventana $w$. El número de eventos resonantes $M$ $M a$ está representado por el área bajo la gaussiana, sin la base $\mathrm{B}$, afectado por el factor $f_{i}$. El número total de eventos $M$ será el área total bajo la curva, entre el centro y $w$. Resulta:

$$
\frac{S}{N}=\frac{M-M a}{\sqrt{M}}=\frac{f_{i} L \sqrt{2} \sigma \operatorname{erf}\left(\frac{w}{\sqrt{2} \sigma}\right)}{\sqrt{L \sqrt{2} \sigma \operatorname{erf}\left(\frac{w}{\sqrt{2} \sigma}\right)+w B}}
$$

donde erf es la función de error, integral de la gaussiana, mientras que $L, B$ y $\sigma$ dependen de la geometría del espectro de energía.

La ventana puede normalizarse como: 


$$
w^{\prime}=\frac{w}{\sqrt{2} \sigma}
$$

Extrayendo las constantes y normalizando la altura tal que $L=1$, conservando $B$ como parámetro, se obtiene:

$$
\frac{S}{N}=K \frac{\operatorname{erf}\left(w^{\prime}\right)}{\sqrt{\operatorname{erf}\left(w^{\prime}\right)+B w^{\prime}}}
$$

Encontrar la ventana óptima equivale a encontrar el valor de $w^{\prime}$ para el cual tiene un máximo dicha expresión.

En la Figura 33 puede verse la dependencia de la relación señal a ruido con el parámetro $B$.

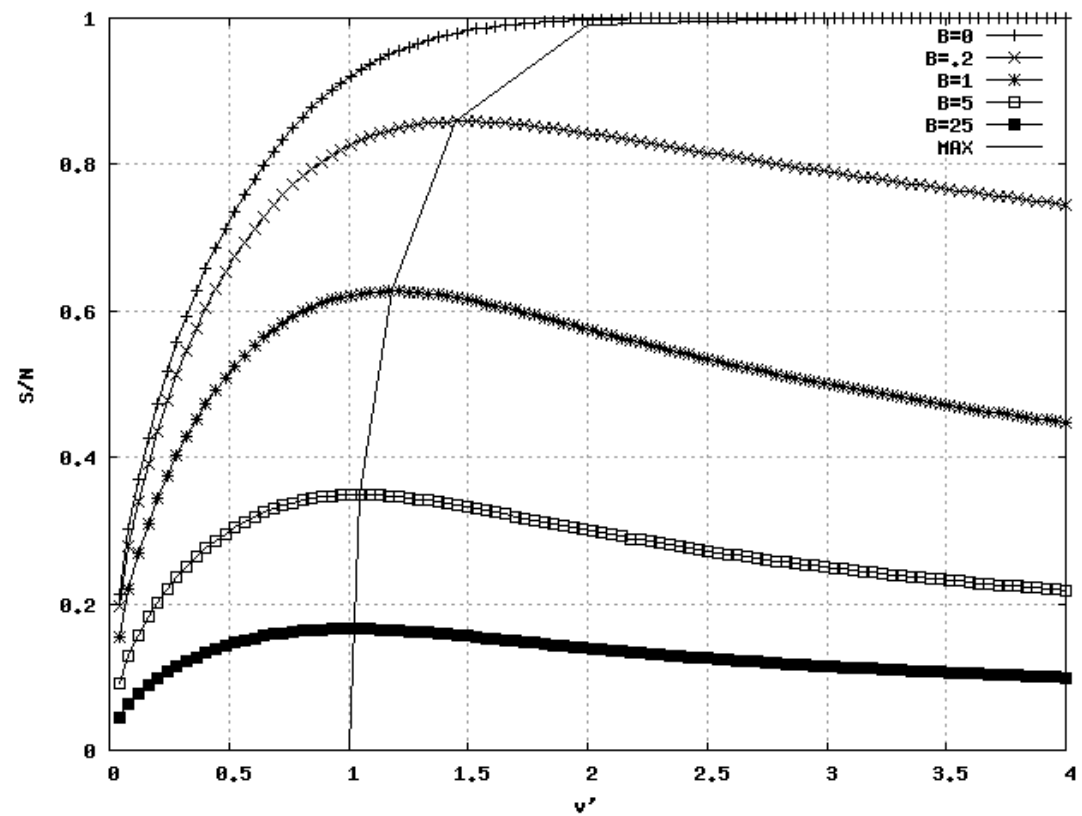

Figura 33: Relación señal a ruido $(K=1)$ en función del tamaño de la ventana con B como parámetro.

De esta gráfica pueden extraerse algunas conclusiones. La primera, y más obvia, es que cuando la base sea nula $(B=0)$ la ventana óptima será totalmente abierta, ya que como sólo hay fotones resonantes no será necesaria una ventana. Otra conclusión interesante, y no tan evidente, es que existe una ventana mínima $w^{\prime}=1$ para el caso de fondo muy grande. $\mathrm{O}$ sea que $w= \pm \sqrt{2} \sigma$ será la ventana óptima para un $B$ muy grande, donde $\sigma$ es la desviación estándar del pico. Esta ventana comprende el 84\% de los eventos (mayor que FWHM, que incluye el $75 \%$ ). Este punto se encuentra aproximadamente a un tercio de la altura total de la gaussiana (FWHM está a 0.5 y $\sigma$ a 0.68 ). 


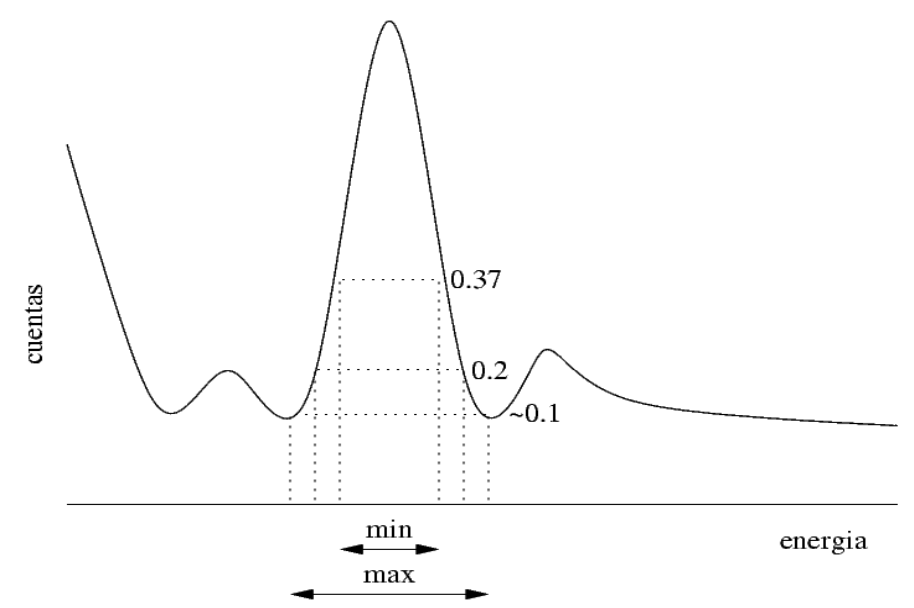

Figura 34: Rango de la ventana óptima en la práctica.

En la práctica, como muestra la Figura 34, el nivel de energías de interés se encuentra próximo a dos niveles de rayos $\mathrm{X}$ que limitan la aplicación de estos resultados hasta cierto punto:

i. En el caso de $B<<L$ la ventana puede abrirse hasta los valles a ambos lados del nivel de interés. Abrirla más allá de este límite implica una desmejora de la relación señal a ruido. Puede considerarse que este punto se encuentra a un $10 \%$ de la altura total del pico.

ii. En el caso de $B>>L$ puede utilizarse la ventana mínima al tercio de la altura.

iii. En los casos intermedios puede calcularse la ventana óptima o utilizarse la Figura 33. Esta no es una tarea simple ya que requiere de la utilización de un multicanal para relevar el espectro con precisión, pero tiene la ventaja de que sólo debe hacerse una vez para cada fuente.

Más allá de la complejidad de la calibración, un resultado interesante que se deriva de este análisis es que en términos de eficiencia no se justifica utilizar un criterio demasiado estricto de selección de los eventos. Utilizar una ventana muy cerrada no garantiza la calidad de los espectros. Incluso existe un límite más allá del cual nunca es conveniente cerrar la ventana.

El análisis presentado respecto de la ventana óptima no implica restricciones a la tasa de eventos a utilizar. Una vez seleccionada la ventana, la fuente puede acercarse o alejarse del absorbente a fin de conseguir el conteo apropiado. Esta acción no modifica las proporciones calculadas. En las próximas secciones se tratan algunos criterios que pueden utilizarse para seleccionar apropiadamente dicha tasa de trabajo.

\subsection{Tiempo muerto en la etapa de detección}

Según la expresión (12) es deseable incrementar la tasa de conteo del experimento, con el objetivo de disminuir el tiempo de medida. Esto se consigue acercando la fuente radiactiva al material en estudio tanto como las consideraciones de ángulo sólido lo permitan. Pero en todo sistema de conteo existen limitaciones impuestas por el tiempo muerto del mismo. 
Se entiende por tiempo mиerto al tiempo que demora la etapa de detección en estar lista para un nuevo evento, luego de haber detectado y procesado el evento anterior. El tiempo muerto del conjunto estará determinado por el componente de mayor tiempo muerto en la etapa. Cuanto mayor es el tiempo muerto, dada una determinada tasa de conteo, mayor es la probabilidad de que un nuevo evento ocurra dentro del tiempo muerto del evento anterior. De la misma forma, dado un tiempo muerto, mayor será la proporción de eventos perdidos cuanto mayor sea la tasa de conteo.

Existen dos posibles comportamientos del sistema respecto del tiempo muerto, dependiendo si éste se redispara prolongando el efecto (sistema extendible) o no. Ambos casos han sido estudiados frecuentemente en la bibliografía [Müller 1973] [Leo 1994] y se presentan a continuación a fin de establecer un punto de partida para el caso particular de interés. Los dos casos se estudian por separado suponiendo sistemas con tiempo muerto $\tau$ constante para todos los eventos. Este criterio es aplicable a un amplificador con conformación pseudo-gaussiana, como los utilizados en experimentos Mössbauer.

En el caso extendible la llegada de un evento durante el tiempo muerto prolonga dicho tiempo muerto a partir del instante de llegada, como se ilustra en la Figura 35. Si la tasa de arribo es suficientemente alta, los tiempos muertos se superponen, prolongando el tiempo durante el cual nuevos eventos no son procesados, pudiendo llegar eventualmente a contar cero ${ }^{4}$. $\mathrm{Al}$ contrario, un sistema no extendible permanece insensible a la llegada de nuevos eventos durante el tiempo muerto, estando listo para una nueva cuenta luego de transcurrido el tiempo $\tau$, sin importar si durante ese tiempo ocurrieron o no eventos. Las consecuencias pueden cuantificarse en ambos casos.

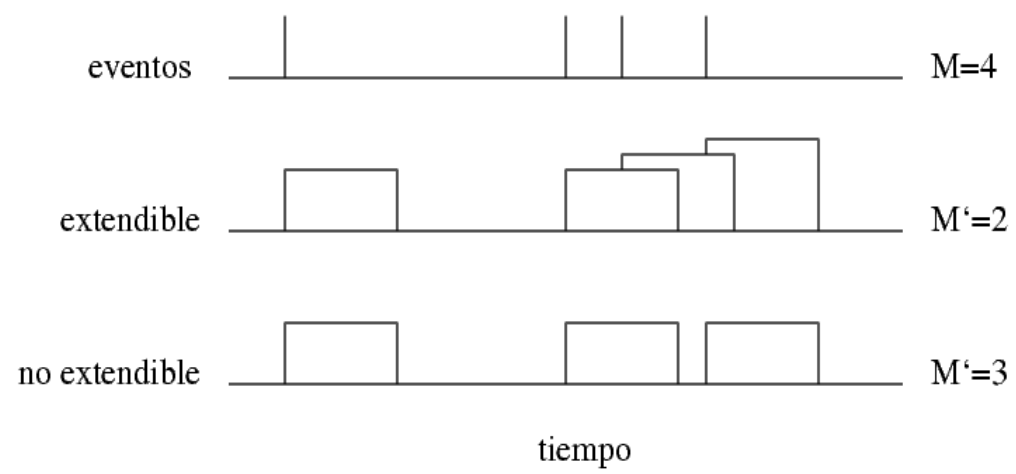

Figura 35: Efecto del tiempo muerto para un sistema extendible y para uno no extendible

Consideraremos primero el caso no extendible. Sea un arribo aleatorio de eventos de media $m$ cuentas por segundo. El sistema registra $M$ cuentas en un tiempo $T$. Si el tiempo es lo suficientemente grande, se cumple que $M=m T$. Considerando un tiempo muerto por evento de $\tau$ segundos, resulta un tiempo muerto total de $M \tau$ durante el período $T$. Durante este tiempo muerto total se habrán perdido $m M \tau$ eventos. Entonces el número de eventos registrados será:

$$
M^{\prime}=m^{\prime} T=m T-m^{\prime} M \tau
$$

4 Por este motivo a este tipo de sistemas suelen ser denominados sistemas paralizables. 
donde $m$ es la tasa real, $m^{\prime}$ la tasa observada y $\tau$ el tiempo muerto del sistema.

Resulta así una tasa observada dada por:

$$
m^{\prime}=\frac{m}{1+m \tau}
$$

Conociendo el tiempo muerto del sistema, esta expresión permite predecir la proporción de pérdidas debido al tiempo muerto. De la misma manera permite obtener la tasa verdadera a partir de la tasa observada.

El estudio de los tiempos muertos extendibles es un poco más complicado. En este caso sólo serán medidos aquellos eventos que lleguen a intervalos más grandes que $\tau$. La distribución de intervalos de tiempo entre eventos aleatorios provenientes de una fuente con distribución de probabilidades exponencial, con tasa media $m$ puede expresarse como:

$$
P(t)=m e^{-m t}
$$

La probabilidad de que el intervalo entre eventos sea mayor que el tiempo muerto será:

$$
P(t>\tau)=m \int_{\tau}^{o o} e^{-m t} d t=e^{-m \tau}
$$

El número de eventos observados en un intervalo $T$ está determinado por la fracción $e^{-m \tau}$ del valor real dado por $m T$. Resulta así una tasa observada dada por la expresión:

$$
m^{\prime}=m e^{-m \tau}
$$

Esta expresión tiene un máximo en $m_{M A X}=1 / \tau$, a partir del cual un aumento de la tasa real produce una disminución de la tasa observada.

En la Figura 36 puede verse una comparación del efecto de un mismo tiempo muerto para el caso extendible y para el no extendible, ambos comparados con un sistema ideal sin tiempo muerto.

Es importante notar que en ambos casos el error introducido es sistemático, pudiéndose calcular $m$ conociendo $m^{\prime}$, con la condición de conocer exactamente el valor del tiempo muerto. En el caso extendible existen dos soluciones que deben ser diferenciadas, y las mismas deben ser obtenidas por métodos numéricos.

Conocer exactamente el tiempo muerto de un sistema no es una tarea simple. En general sólo puede obtenerse una estimación del mismo. En la bibliografía se mencionan algunos métodos experimentales que tienen aplicación en casos específicos [Leo 1994].

Estos dos modelos son los utilizados generalmente para cuantificar los efectos del tiempo muerto en cualquier sistema de conteo. En el caso de la espectroscopia Mössbauer, a primera vista, el efecto de disminución de la tasa parece no ser de importancia, ya que casi siempre 
puede acercarse un poco más la fuente al absorbente para reponer el conteo deseado y compensar las pérdidas por tiempo muerto ${ }^{5}$.

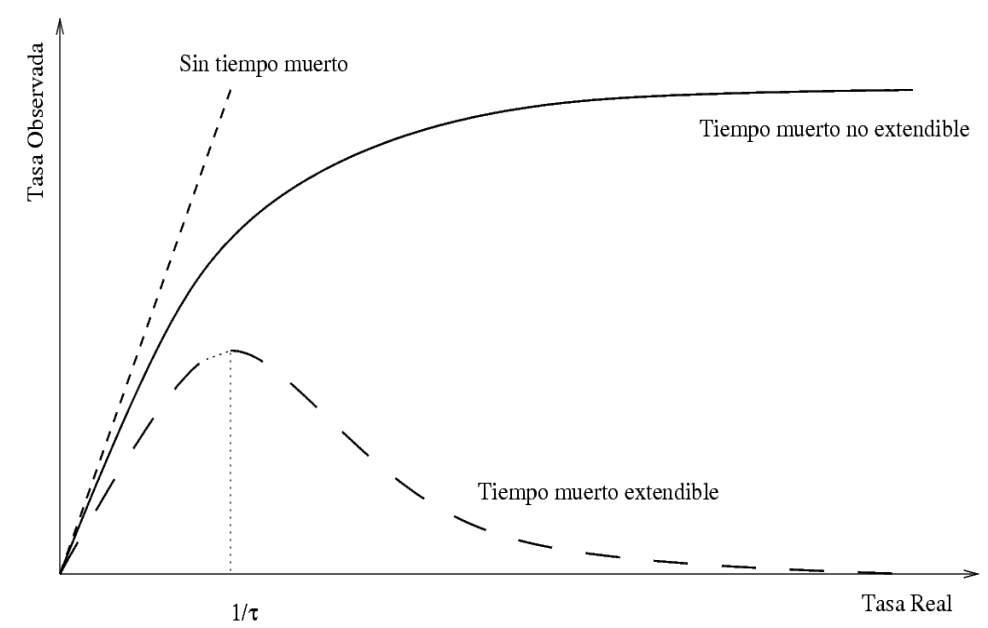

Figura 36: Tasa observada $m^{\prime}$ para un determinado tiempo muerto en función de la tasa real $\mathrm{m}$.

Sin embargo, los espectros Mössbauer tienen una particularidad en este aspecto, que no es tratada en la bibliografía relacionada con instrumentación nuclear, posiblemente por tratarse de una característica propia de este experimento. Pero debe tenerse en cuenta que el efecto que se describe a continuación tiene un gran peso sobre la definición presentada de la relación señal a ruido del espectro.

Dado que la tasa de conteo puede variar un $20 \%$ o más entre una línea de absorción y el fondo del espectro (lo que llamamos efecto $f$ ), la consecuencia de las pérdidas por tiempo muerto será diferente en ambos casos. En el caso del fondo el porcentaje de eventos perdidos será mayor, debido a que la tasa es mayor. Esto se refleja en una compresión del espectro o pérdida del efecto medido, como muestra la Figura 37.

Esta compresión puede magnificarse sobre el efecto Mössbauer. Considerando las pérdidas por tiempo muerto tanto en la línea como en el fondo, resulta un efecto medido $f^{\prime}$ en el caso no extendible, a partir de la ecuación (6), dado por la expresión:

$$
f^{\prime}=1-\frac{\frac{(1-f) m}{1+(1-f) m \tau}}{\frac{m}{1+m \tau}}=\frac{f}{1+(1-f) m \tau}
$$

Para el caso extendible resulta:

$$
f^{\prime}=1-\frac{(1-f) m e^{-(1-f) m \tau}}{m e^{-m \tau}}=1-(1-f) e^{f m \tau}
$$

5 En el caso de un sistema extendible existe un límite para el aumento de la tasa dado por $m_{\text {MAX }}$. 


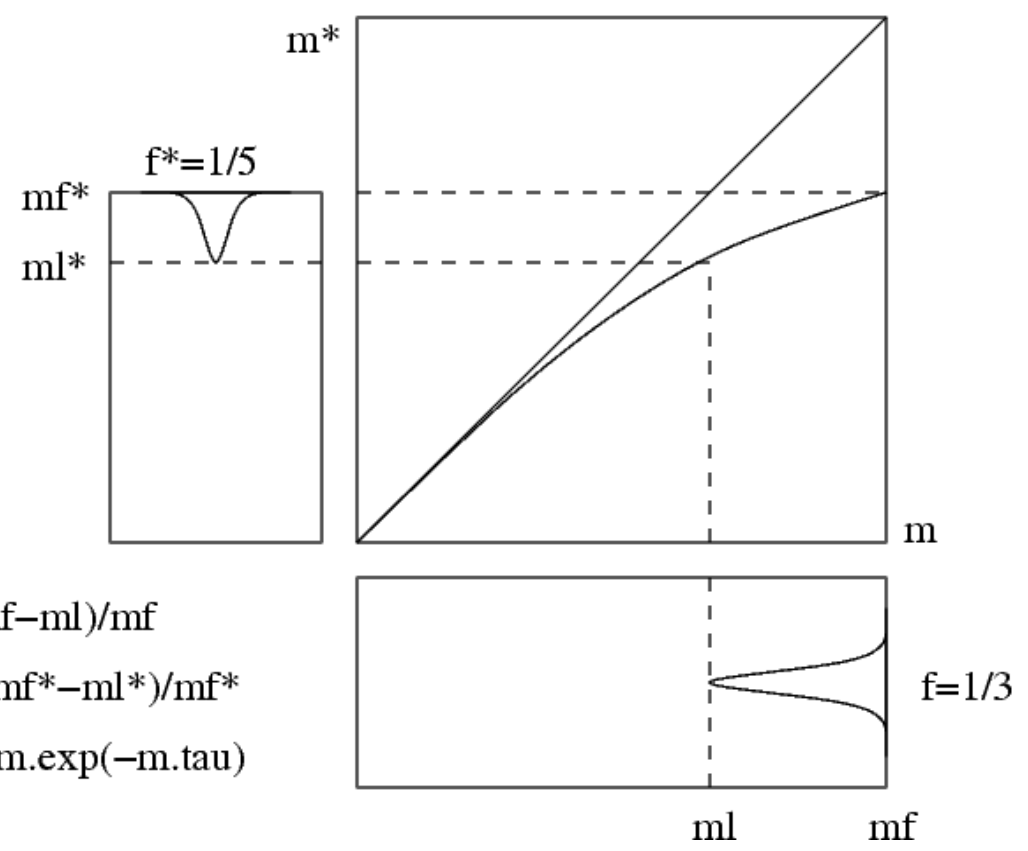

Figura 37: Efecto de compresión asimétrica del espectro causado por el tiempo muerto en la etapa de detección.

Puede verse que en ambos casos el efecto resultante depende sólo de $f$ y de la relación que existe entre el tiempo muerto y la tasa. Puede definirse una relación que muestre cuánto pesa el tiempo muerto comparado con el tiempo medio entre eventos, a la cual llamaremos relación de tiempo muerto:

$$
\theta=\frac{\tau}{1 / m}=\tau m
$$

En la Figura 38 puede verse la disminución del efecto medido en función de la relación de tiempo muerto para ambos casos.

Por ejemplo, dados un tiempo muerto de 10 us y una tasa de $20000 \mathrm{c} / \mathrm{s}$, si el efecto es de 0.2 resulta un efecto medido de 0.172 para el caso no extendible y de 0.167 para el caso extendible.

Resumiendo, en el caso de un espectro Mössbauer no solo disminuye el conteo a causa del tiempo muerto, sino que además se produce una disminución del efecto y por lo tanto una doble desmejora en la relación señal a ruido. Como se podía suponer, los efectos de un tiempo muerto extendible son más evidentes que los de un tiempo muerto no extendible. Pero en ambos casos la compresión del espectro es un punto importante a tener en cuenta en el momento de seleccionar la tasa de trabajo.

En consecuencia es importante encontrar el tiempo muerto total de la etapa de detección. Éste será aproximadamente igual al máximo tiempo muerto de todos los componentes de la etapa. 


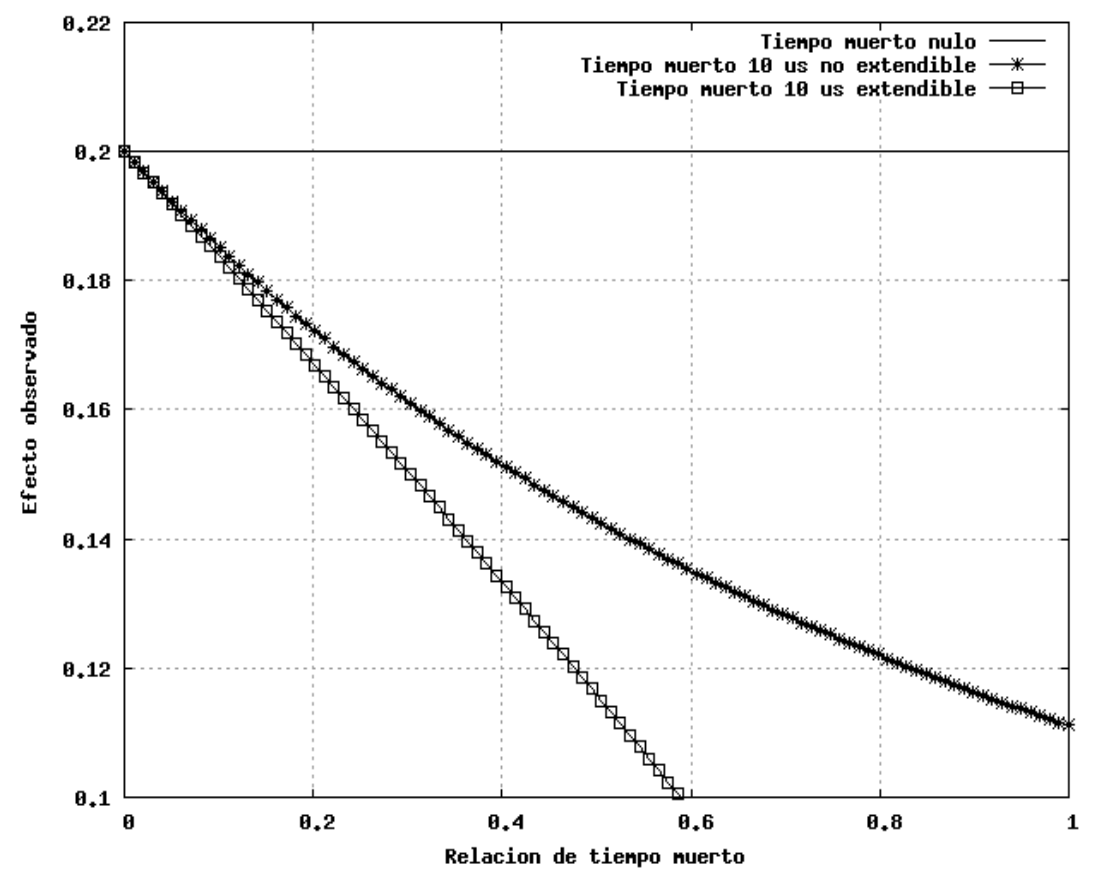

Figura 38: Disminución del efecto medible en función de la tasa para un sistema con $f=0.2$ y $\tau=10$ us, para los casos extendible y no extendible.

A continuación de identifican el tipo (extendible o no extendible) y la magnitud de los tiempos muertos presentes.

i. El conjunto detector-preamplificador es extendible. La señal de carga proveniente del detector es integrada por el preamplificador. La constante de tiempo de descarga del integrador (del orden de las decenas de microsegundos) es relativamente grande, pero debe mantenerse así para no desmejorar la amplitud del pulso. Este tiempo muerto es eliminado por el amplificador, que genera un pulso conformado proporcional a la derivada de esta exponencial (flanco ascendente).

ii. La salida del amplificador es también extendible. El ancho de los pulsos de salida depende de la constante de shaping utilizada. Por ejemplo, para el modelo 572 de Ortec puede variar entre 3 y 70 us. Este valor se seleccionará de acuerdo al ruido presente a la entrada del amplificador. Debe tenerse en cuenta que este dispositivo amplifica tanto a los eventos de interés como a los de fondo y a los provenientes de transiciones de otras energías.

iii.Los discriminadores comerciales están diseñados de forma de presentar tiempos muertos no extendibles. Por ejemplo, el modelo 551 de Ortec especifica un tiempo muerto de $800 \mathrm{~ns}$ en su salida TTL y $200 \mathrm{~ns}$ en la salida NIM.

iv. El contador es un elemento puramente digital. Utilizando lógica rápida puede diseñarse sin inconvenientes para operar con tiempos muertos no extendibles, menores que la décima de microsegundo.

De lo expuesto resulta que el dispositivo crítico respecto del tiempo muerto es el amplificador, cuyo efecto de apilamiento puede modelarse como un tiempo muerto 


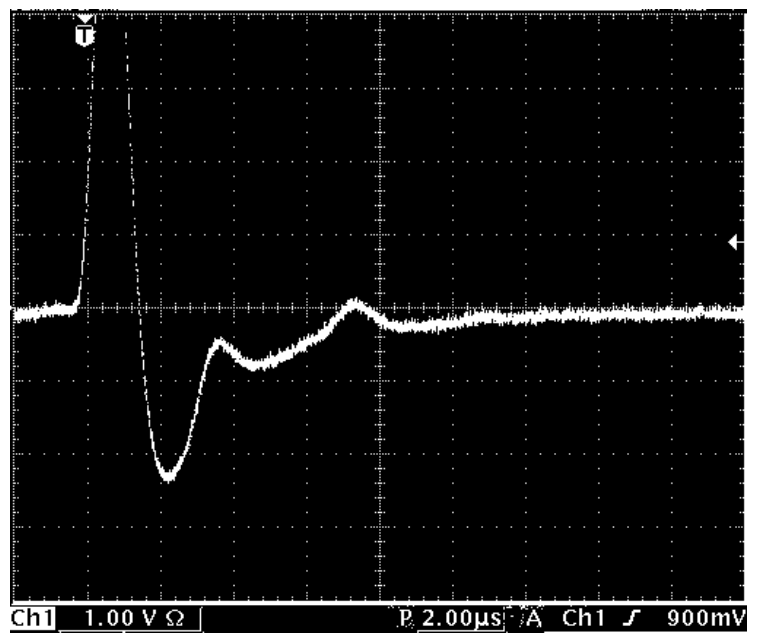

Figura 39: Salida bipolar de un amplificador

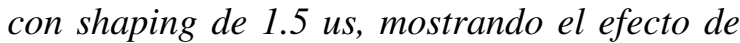
apilamiento en el caso de un evento de gran energía seguido de dos de energía menor.

extendible, como muestra la Figura 39. En esta figura pueden verse tres pulsos bipolares superpuestos a la salida del amplificador, de los cuales el segundo corresponde a un fotón de 14.4 KeV Este evento no entrará en la ventana del discriminador por estar superpuesto con un pulso de $136 \mathrm{KeV}$ En este ejemplo puede verse cómo la presencia de fotones de gran energía (que no producen absorción resonante) puede desmejorar la eficiencia de la etapa de detección, al punto de que sólo combinaciones fortuitas de pulsos tendrán la altura necesaria para entrar en la ventana del discriminador. Dicha combinación estará asociada a eventos con muy baja probabilidad de resonar, disminuyendo el efecto de absorción.

En conclusión, el pulso de salida del amplificador debe hacerse lo más angosto posible para evitar el apilamiento. Pero para que la salida pueda ser muy rápida el amplificador debe tener un gran ancho de banda, lo que desmejora la relación señal a ruido del amplificador. Esto repercute en la calidad del espectro disminuyendo la calidad del ventaneo. Desmejora así el efecto medible y por lo tanto la relación señal a ruido del espectro obtenido.

Concluyendo, el ancho total de los pulsos de salida del amplificador puede considerarse representativo del tiempo muerto de la etapa completa. La selección del shaping del amplificador presenta un compromiso entre calidad de la altura del pulso y tiempo muerto.

\subsection{Cálculo de la tasa óptima}

Por lo expuesto en la sección anterior, una tasa de eventos muy alta puede producir una compresión del espectro, disminuyendo el efecto medido y aumentando así el tiempo necesario para obtener una determinada relación señal a ruido. Por otro lado, si la tasa es muy baja, aumenta el ruido estadístico y por lo tanto aumenta también el tiempo. Si se desea obtener un espectro con una relación señal a ruido determinada en el menor tiempo posible, considerando los efectos del tiempo muerto, debe encontrarse el mínimo de la expresión del tiempo total de medida $T$ en función de la tasa $m$. Se puede obtener así la tasa óptima $m_{O P T}$.

Para un dado efecto y una cierta cantidad de canales, si el amplificador tiene un tiempo 
muerto extendible $\tau$ y a la entrada observa una tasa $m$, el tiempo necesario para acumular una estadística lo suficientemente grande para satisfacer una determinada relación señal a ruido estará determinado por la ecuación (12) con los efectos de tiempo muerto representados por la ecuación (20) en lo que se refiere a disminución de la tasa, y por la ecuación (22) en lo relativo a la disminución del efecto. Resulta entonces, combinando ambas ecuaciones, que el tiempo total de medida necesario para obtener una determinada relación señal a ruido $S / N$ en un cierto número de canales $N_{c}$ estará determinado por la expresión:

$$
T=\left(\frac{S / N}{1-(1-f) e^{f m \tau}}\right)^{2} \frac{N_{c}}{m e^{-m \tau}}
$$

La tasa óptima será el valor de $m$ para el cual se hace mínimo el tiempo $T$. Esta expresión tiene un mínimo para una tasa menor que la $m_{M A X}$ predicha por la expresión (20) en el caso de tiempos muertos extendibles. En la Figura 40 se muestra un ejemplo en el cual se busca obtener una relación señal a ruido de $40 \mathrm{~dB}$ en 256 canales considerando un efecto $f$ de $20 \%$ y un tiempo muerto $\tau$ de 10 us. El mínimo tiempo de medida se consigue con una tasa de 30000 $\mathrm{c} / \mathrm{s}$ en el amplificador y es de casi una hora y media. Esta tasa es mucho menor que el $m_{M A X}$ de $100000 \mathrm{c} / \mathrm{s}$. Por la expresión (20) se obtiene una tasa medida $m^{\prime}$ de $22000 \mathrm{c} / \mathrm{s}$ y por la ecuación (22) un efecto medido $f^{\prime}$ del $15 \%$.

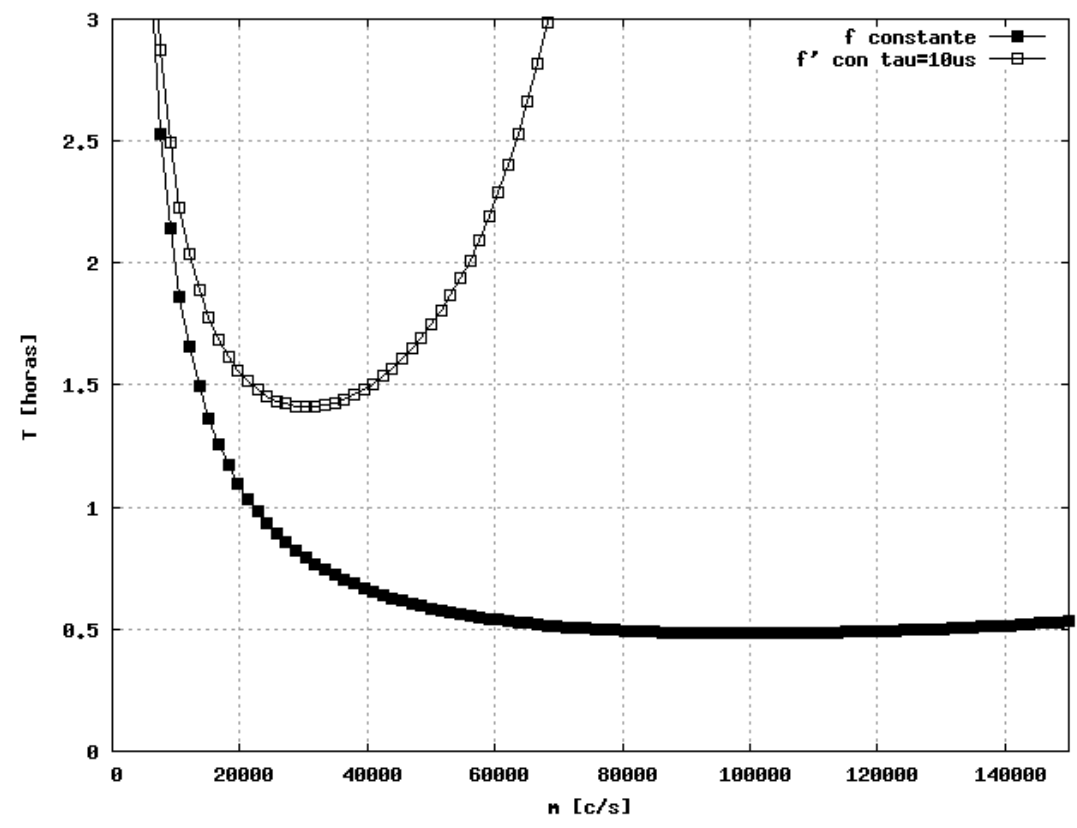

Figura 40: Tasa óptima en el caso $f=0.2, \quad t m=10 u s, \quad S / N=100$, $N c=256$.

Si la tasa es más baja que el óptimo es necesario un tiempo adicional para colectar la estadística necesaria, y si es más alta la compresión de la señal requiere un menor ruido y por lo tanto un mayor tiempo de medida. Esto no significa que el espectro obtenido utilizando la tasa óptima sea el mejor para todos los casos. Esta es la condición que optimiza la relación señal a ruido del espectro, lo cual es útil, por ejemplo, para obtener la posición de la línea (a qué velocidad se produce la máxima absorción resonante). Pero si lo que se busca es obtener 
el factor $f$ (profundidad de la línea) será más conveniente utilizar una tasa menor, tal que $f^{\prime}$ se aproxime lo más posible a $f$.

Hasta el momento el análisis se realizó suponiendo un único tipo de eventos de tasa $m$ y que todos ellos tienen una probabilidad idéntica de resonar. Esto sería como considerar en el caso del hierro que lo único que recibe el detector son fotones de $14.4 \mathrm{KeV}$ Y sabemos que eso no es así. El detector recibe tanto fotones de $14.4 \mathrm{KeV}$ como de $136 \mathrm{KeV}$, más un fondo, rayos $\mathrm{X}$, scattering, etc. Cuando se intercala un discriminador y se seleccionan sólo los de 14.4 $\mathrm{KeV}$, baja drásticamente la tasa que detecta el contador, pero se mantiene mucho más alta la tasa en el amplificador. Esto hace más crítico el tiempo muerto del amplificador, considerando que los fotones que no son de $14.4 \mathrm{KeV}$ son también abundantes.

Consideremos ahora un modelo simplificado que tenga en cuenta el efecto de los fotones no resonantes en el amplificador. El tiempo muerto para estos eventos es igual que para los resonantes, estando determinado por el shaping del amplificador. Sea entonces una tasa $m_{T}$ a la salida del preamplificador (entrada del amplificador), que tendrá dos componentes: fotones con la amplitud necesaria para atravesar la ventana con una tasa $m_{V}$, y otros fotones con una tasa $m_{O}$, tal que:

$$
m_{T}=m_{V}+m_{O}
$$

Debido al tiempo muerto extendible del amplificador, efecto cuantificado en la ecuación (20), y considerando los procesos aleatorios independientes, a la entrada del discriminador resulta una tasa $m_{T}^{\prime}$ dada por la expresión :

$$
m_{T}^{\prime}=\left(m_{V}+m_{O}\right) e^{-m_{T} \tau}=m_{V} e^{-m_{T} \tau}+m_{O} e^{-m_{T} \tau}
$$

Al aplicar la ventana en el discriminador se elimina el segundo término y se obtiene a la salida una tasa observada dada por la expresión:

$$
m_{v}{ }^{\prime}=m_{V} e^{-m_{T} \tau}
$$

En este punto resulta de utilidad definir un nuevo parámetro $Q$ de la etapa de detección, dado por la siguiente proporción:

$$
Q=\frac{m_{v}}{m_{T}}
$$

Este representa la proporción de eventos que serán seleccionados por el discriminador, frente al total de eventos que produce apilamiento en el amplificador. Para obtener este parámetro sería necesario obtener un espectro completo de amplitudes a la salida del amplificador y dividir el área bajo la curva en la ventana por el área total del espectro. Una aproximación puede obtenerse calculando la relación que existe entre el conteo cuando está activa la ventana a utilizar, y el conteo que se produce con una ventana totalmente abierta. Un valor experimental razonable para una fuente comercial está entre $1 / 2$ y 1/3. 
Utilizando el factor $Q$ la tasa observada puede expresarse como:

$$
m_{v}^{\prime}=m_{v} e^{-\frac{m_{v}}{Q} \tau}
$$

Así $m_{v}^{\prime}$ es la tasa medida a la salida del discriminador, cuyo máximo depende de $Q$, además del tiempo muerto.

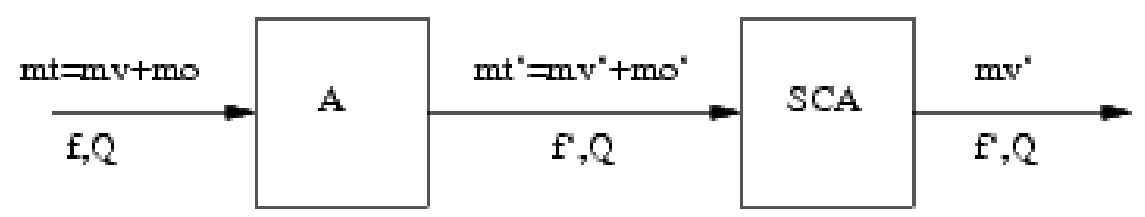

Figura 41: Nomenclatura para las tasas involucradas en la etapa de detección.

Aplicando el mismo concepto al efecto de compresión por tiempo muerto representado por la ecuación (22), resulta que ante la presencia de un mayor número de eventos en el amplificador el efecto resultante debe calcularse como:

$$
f^{\prime}=1-(1-f) e^{f \frac{m_{v}}{Q} \tau}
$$

Reescribiendo la ecuación (24) del tiempo total de medida, a fin de contemplar el efecto de los eventos no resonantes, utilizando las ecuaciones (29) y (30):

$$
T=\left(\frac{S / N}{1-(1-f) e^{f \frac{m_{v}}{Q} \tau}}\right)^{2} \frac{N_{c}}{m_{v} e^{-\frac{m_{v}}{Q} \tau}}
$$

Esta expresión permite encontrar la tasa $\mathrm{m}_{\mathrm{v}}$ a la entrada del amplificador que minimiza el tiempo de medida necesario para obtener una determinada relación señal a ruido. A partir de este valor obtenido, utilizando la expresión (29) puede obtenerse la tasa que debe utilizarse en el discriminador, y utilizando (30) se obtiene el efecto resultante.

La tasa óptima y el tiempo total de medida, como muestra la Figura 42, dependen fuertemente del factor $Q$. Considerando un valor de $1 / 3$ para el ejemplo anterior (donde $f=0.2, \tau=10 \mathrm{us}, S$ / $N=100, N c=256$ ) la tasa óptima se reduce de 30000 a $10000 \mathrm{c} / \mathrm{s}$, resultando en un incremento proporcional del tiempo de medida.

Este análisis puede simplificarse para un experimento particular, considerando que el efecto $f$ es intrínseco del absorbente en estudio, por lo que no es necesario conservarlo como parámetro. A tal efecto puede volver a definirse la relación de tiempo muerto de la expresión (21) como:

$$
\theta=\frac{m \tau}{Q}
$$




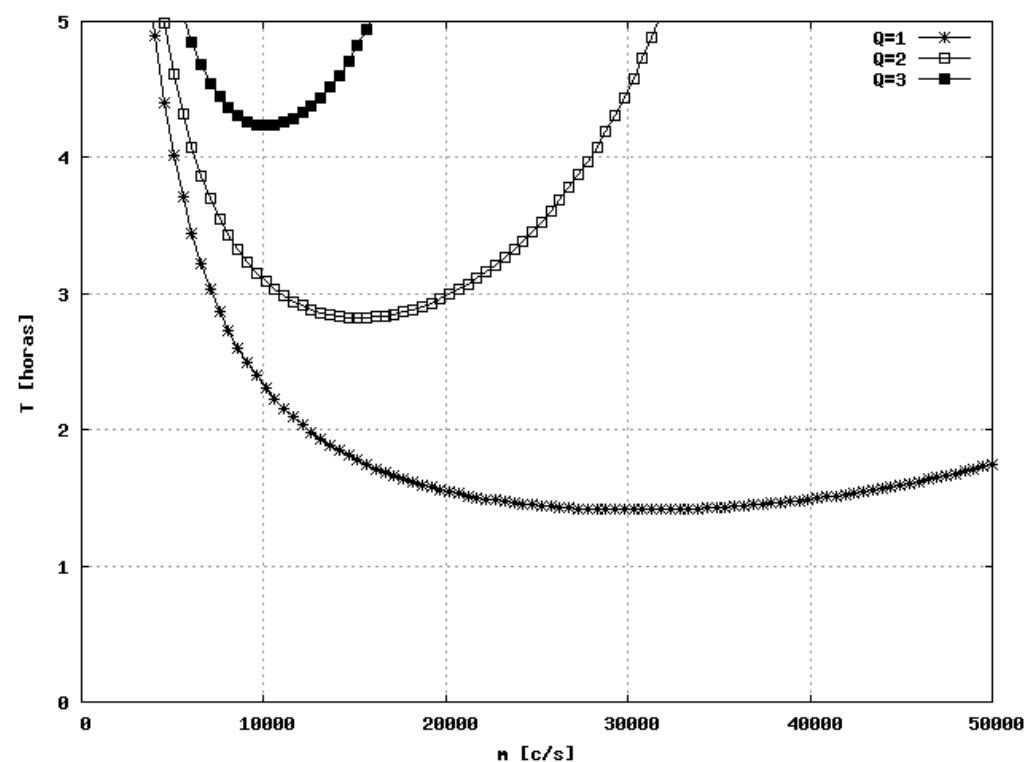

Figura 42: Disminución de la tasa óptima con el factor $Q$ para un tiempo muerto de 10 us.

La expresión del tiempo de medida puede escribirse como:

$$
T=\left(\frac{S / N}{1-(1-f) e^{f \theta}}\right)^{2} \frac{N_{c}}{m e^{-\theta}}
$$

En esta expresión puede verse que para cada valor de $f$ existe un $\theta_{O P T}$ y un $f^{\prime}$ resultante.

Por ejemplo, para un $f$ de 0.2 , que es el caso del hierro a temperatura ambiente con espesor óptimo y ventana óptima, $\theta_{O P T}$ vale 0.3 . Pueden usarse las aproximaciones de la Tabla 1 para calcular la tasa óptima y el tiempo de medida.

\begin{tabular}{|c|c|}
\hline CASO OPTIMO PARA EL HIERRO $(f=0.2)$ \\
$m=\frac{0.3 Q}{\tau} \quad m^{\prime}=0.74 m \quad f^{\prime}=0.15 \quad T=\frac{60(S / N)^{2} N_{c}}{m}$ \\
\hline
\end{tabular}

Tabla 1: Caso óptimo para el hierro a temperatura ambiente

En dichas expresiones $\tau$ es el tiempo muerto del amplificador y $S / N$ y $N_{c}$ dependen del tipo de espectro que se desee obtener. Es importante notar que si se desean predecir estos resultados es necesario disponer del factor $Q$, que depende de la geometría del espectro y de la ventana utilizada.

\subsection{El procedimiento de calibración propuesto}

Se procederá a continuación a ordenar la información presentada a lo largo del capítulo en 
forma de un procedimiento de calibración recomendado para la etapa de detección. Este procedimiento obtiene la máxima eficiencia del conjunto, tal como fue definida en función del tiempo: se configura la etapa con los parámetros que garantizan una máxima relación señal a ruido para un determinado tiempo de medida.

1) Utilizar el espesor óptimo para la muestra en estudio, en caso de ser posible. Esta optimización en en la misma dirección que el resto de las consideraciones que siguen a continuación.

2) Minimizar el ruido eléctrico en la etapa. El preamplificador debe montarse lo más cerca posible del detector, de ser posible sin cables. Los conectores deben garantizar óptimos contactos y blindajes. El cable que transporta la señal analógica desde el preamplificador al amplificador, que usualmente está montado en el rack NIM, debe ser lo mas corto posible. Si el ambiente no es ruidoso y se cuida la conexión entre el amplificador y el discriminador puede utilizarse el shaping mínimo del amplificador, resultando en el mínimo tiempo muerto $\tau=3 u s$ que puede obtenerse con instrumentación tradicional. Deben utilizarse discriminadores y contadores digitales con tiempos muertos mucho menores que éste, a fin de no desmejorar la performance de la etapa.

3) Obtener un espectro de amplitudes a la salida del amplificador. Este debe realizarse con un analizador multicanal, con la muestra colocada, a velocidad constante alta para que no exista absorción resonante (mayor que $8 \mathrm{~mm} / \mathrm{s}$ para el hierro), y a baja tasa para evitar los efectos del tiempo muerto (menor que $10000 \mathrm{c} / \mathrm{s}$ ). Del espectro pueden obtenerse los parámetros $\sigma$, B y L.

4) Calcular la ventana óptima. Para ello deben utilizarse en la expresión (13) los parámetros $\sigma$, B y L obtenidos en el punto anterior. Además, con la nueva ventana puede obtenerse una aproximación del parámetro $Q$. Puede usarse la relación entre el conteo con ventana y el conteo con la ventana totalmente abierta. Para fijar los valores alto y bajo de discriminación puede utilizarse un osciloscopio con persistencia o realizar iteraciones con el multicanal, siendo más recomendable esta última técnica a pesar de que requiere una mayor cantidad de tiempo.

5) Calcular la tasa óptima. A la entrada del amplificador es el valor de $m_{v}$ que minimiza la expresión (31). Puede calcularse utilizando $Q$ y $\tau$. La tasa óptima en el contador se obtiene de la expresión (29). La distancia de la fuente debe ser adecuada observando la tasa en el elemento contador.

6) Calcular el tiempo de medida. Este será el tiempo que se necesita para obtener una determinada relación señal a ruido en un cierto número de canales. Este puede obtenerse directamente de la expresión (31).

7) Calcular el efecto resultante. La desmejora en el efecto puede calcularse con la expresión (30).

A continuación se mostrará un ejemplo práctico que ilustra la utilidad de este método. El procedimiento descripto se aplicó a la etapa de adquisición de un espectrómetro Mössbauer con fuente de $\mathrm{Co}^{57}$ que se encontraba en funcionamiento. Este espectrómetro, luego de innumerables ajustes por prueba y error, había logrado ser catalogado como 'productor de 
espectros de buena calidad'. Se encontró que la ventana del discriminador seleccionaba casi la totalidad de la línea de $14.4 \mathrm{KeV}$, prácticamente hasta el límite impuesto por los valles laterales. Esto es coincidente con el cálculo de ventana óptima, considerando que se midió un $Q$ de aproximadamente 0.2. El shaping del amplificador estaba al mínimo (0.5 us), lo cual es coincidente con los criterios presentados de minimización de ruido. Se estimó entonces un tiempo muerto de 3 us. Los tiempos muertos de los componentes digitales eran mucho menores, ya que se utilizaba un discriminador Ortec con salida NIM y elementos contadores construidos con lógica rápida. El cálculo de la tasa óptima según el método propuesto arroja un valor de 18000 cuentas por segundo en el discriminador. El espectrómetro estaba operando a 17500. La calibración del espectrómetro había requerido un gran número de pruebas y la toma de varios espectros completos de calibración. El método de optimización propuesto insume sólo algunos minutos.

Resumiendo, a lo largo de este capítulo se han presentado los puntos sensibles de optimización de la etapa de detección de un espectrómetro Mössbauer. Ante la ausencia de un método formal de calibración de la misma y ante la multiplicidad de parámetros que pueden modificarse y que tienen influencia sobre los resultados obtenidos, se procedió a desarrollar un método de optimización para esta rama. El método se basa en una definición de la relación señal a ruido del espectro resultante, parámetro que en la mayoría de los casos puede utilizarse para representar los recursos a optimizar en la medición. El procedimiento propuesto es de puesta en práctica sencilla y puede utilizarse en la mayoría de las experiencias que involucren una etapa de detección nuclear lenta, ya sea que se trate de experimentos Mössbauer de velocidad constante programable (como los que se intentarán optimizar a continuación), o de experimentos de aceleración constante, o de cualquier otro experimento que requiera la medición de la energía de los eventos detectados. 


\section{Capítulo 4}

\section{La etapa de accionamiento a velocidad programable}

En este capítulo se presentarán los avances realizados respecto del accionamiento a velocidad constante de los transductores electromecánicos utilizados en los espectrómetros Mössbauer.

Recordemos que estos dispositivos, cuyos detalles constructivos fueron presentados en el Capítulo 2, se utilizan para producir el barrido Doppler de los fotones emitidos por la fuente radiactiva, obteniéndose así una variación de la energía de los mismos. Un buen funcionamiento a velocidad constante de los transductores permitirá implementar un instrumento cuya velocidad de operación pueda ser seleccionada sin intervención manual, independientemente de la velocidad de operación anterior. A este nuevo modo de funcionamiento lo hemos denominado de velocidad programable.

La capacidad de operación automática facilitará el estudio de rangos de energía arbitrarios, adecuados a las necesidades de cada experimento, sin las limitaciones de los espectrómetros de aceleración constante tradicionales, en los cuales el rango de energías en estudio debe ser consecutivo y debe estar centrado en velocidad nula.

La estrategia utilizada para encarar la solución de este problema se basó en una premisa fundamental: el nuevo instrumento debe ser implementado utilizando los transductores electromecánicos disponibles en los laboratorios. El motivo para esta decisión es que se trata de dispositivos costosos, cuyos aspectos de diseño (mayormente mecánicos) se encuentran fuera de los alcances de esta tesis. Si bien se trata de dispositivos diseñados para la técnica de aceleración constante, a lo largo de este capítulo se pondrá especial énfasis en adecuarlos a la nueva técnica con la menor cantidad posible de modificaciones.

Algunos intentos en esta dirección se registran en la bibliografía [Seberini 1988], en los 
cuales se ha ensayado en el transductor algún tipo de realimentación de la posición del eje, lo cual implicó modificaciones mecánicas importantes en el dispositivo, como instalar un sensor mecánico de precisión sobre las partes móviles. Utilizando ese punto de partida, en este capítulo se desea avanzar un paso más en esa dirección, ya que se desea obtener capacidad de velocidad constante programable utilizando los transductores tal cual se encuentran disponibles. De ser posible, se intentará preservar también los módulos de control de velocidad que proveen los fabricantes.

En este capítulo se presentará primero una aproximación clásica al problema, para luego introducir dos técnicas mejoradas que permitieron obtener buenos resultados experimentales.

La aproximación clásica consiste en el diseño de un sistema de control automático realimentado que utiliza técnicas de compensación y feedforward. Previamente se presenta una descripción detallada del problema, junto con la modelización del sistema a controlar.

La primera mejora, propuesta sobre la base del diseño anterior, utiliza una realimentación de posición muy simple, de bajo error de inserción, que requiere mínimas modificaciones al transductor. Esta técnica permite diseñar un controlador con buena ganancia en bajas frecuencias, eliminando las complicaciones derivadas del valor medio no nulo de la referencia. La implementación práctica de esta técnica es simple y el diseño de la electrónica asociada no presenta mayores dificultades.

La segunda aproximación novedosa pone énfasis en la operación con contenido de frecuencia acotado, como mecanismo para aumentar la ganancia de lazo cerrado del sistema. Se pone especial atención en la optimización del recorrido que realiza el eje a lo largo de un ciclo de trabajo. Se intenta minimizar el esfuerzo realizado por el control, buscando una trayectoria óptima de velocidad constante. La implementación de esta técnica no requiere modificaciones al transductor, pudiéndose incluso obtener buenos resultados utilizando los controles de velocidad disponibles.

Finalmente se propone un mecanismo de sincronización indispensable para que la velocidad de operación pueda modificarse en forma automática, consiguiéndose así el modo programable propuesto.

Las técnicas presentadas pueden combinarse a fin de obtener un sistema de máximas prestaciones, cuya implementación y performance se presentará en el próximo capítulo.

\subsection{Consideraciones preliminares}

En los capítulos anteriores se describieron los principales aspectos funcionales de la etapa de accionamiento, cuyo diagrama esquemático se repite por comodidad en la Figura 43. Se procede a continuación con una descripción más detallada del problema de control y de las técnicas utilizadas para el accionamiento a velocidad y aceleración constante.

Desde el punto de vista eléctrico, el transductor tiene una función de transferencia determinada por la relación:

$$
\Phi_{t}(s)=\frac{V_{P}(s)}{V_{D}(s)}
$$




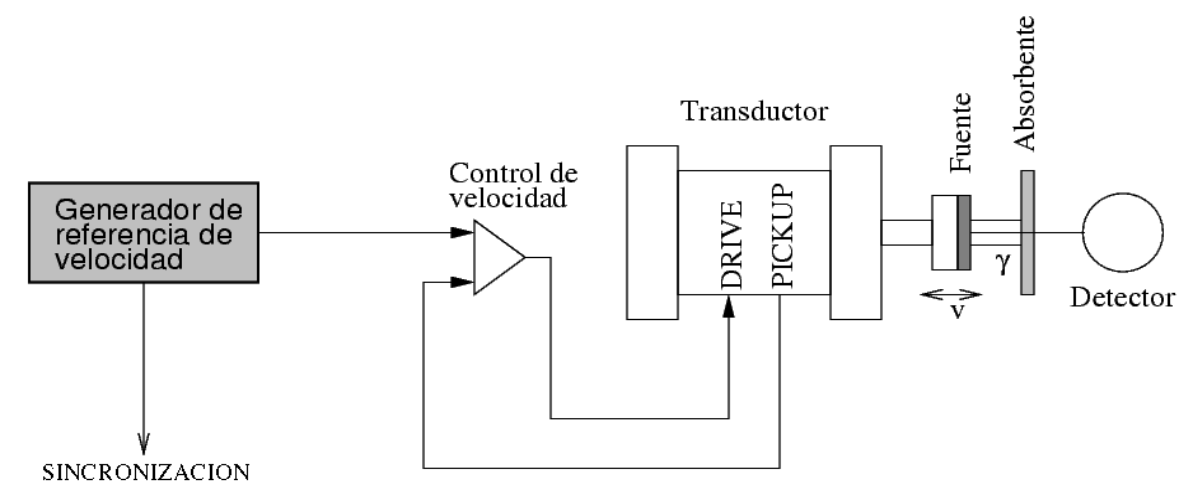

Figura 43: La etapa de accionamiento.

donde $V_{D}$ es la tensión aplicada al arrollamiento de accionamiento y $V_{P}$ la tensión obtenida a la salida de la bobina sensora de velocidad.

La característica a bajas frecuencias del sistema es básicamente derivadora, ya que el accionamiento produce desplazamientos del eje proporcionales a la tensión aplicada en la bobina de accionamiento $V_{D}$. La tensión de salida $V_{P}$ es proporcional a la velocidad del eje, o sea a la derivada primera del desplazamiento. Entonces, a bajas frecuencias, la transferencia puede escribirse como:

$$
\Phi_{t}(s)=s A(s)
$$

donde $A(s)$ es la transferencia libre del efecto derivador. Cuando se aplica una tensión continua a la bobina de accionamiento circula una corriente dentro de un campo magnético perpendicular, proveniente del imán permanente. Se genera entonces una fuerza sobre el arrollamiento solidario al eje, produciéndose un desplazamiento hasta que dicha fuerza iguala a la del resorte de suspensión y se produce el equilibrio. Cuanto más grande sea la tensión aplicada al bobinado, mayor será el desplazamiento respecto del punto de reposo.

Una vez alcanzada la posición de reposo para una determinada tensión aplicada, la salida de la bobina sensora es nula. Sólo se genera una tensión de salida cuando el bobinado de pickup se está moviendo dentro del segundo campo magnético permanente, siendo la tensión inducida proporcional a la velocidad del eje.

El lazo de control se cierra comparando la tensión inducida en la bobina sensora con una tensión proporcional a la velocidad deseada, la cual tiene forma triangular para el caso de aceleración constante o cuadrada para el caso de velocidad constante. La estrategia de control más simple que puede utilizarse es el control proporcional, que se consigue con un amplificador de tensión capaz de entregar la potencia necesaria. Más adelante en este capítulo se presentan el análisis de la estabilidad de dicho sistema, el cálculo del margen de ganancia y una técnica de compensación en serie.

En la Figura 44 pueden verse las formas del desplazamiento, la velocidad y la aceleración cuando se opera el transductor a velocidad o aceleración constante en las condiciones descriptas. Debe tenerse en cuenta que la variable realimentada es la velocidad, lo cual 
implica que el desplazamiento (que es la integral de la velocidad) no tiene un valor medio controlado y puede estar desplazado del centro, como muestra la figura en las gráficas de $s(t)$. Los resortes del sistema de suspensión son los encargados de reponer la posición de reposo, con la dinámica propia de los mismos. Esto puede producir oscilaciones sub-amortiguadas de baja frecuencia en la posición durante el arranque, o bien cuando una perturbación desplace al eje fuera del centro del recorrido. En el modo de velocidad programable este fenómeno se producirá también ante un cambio abrupto de la referencia.
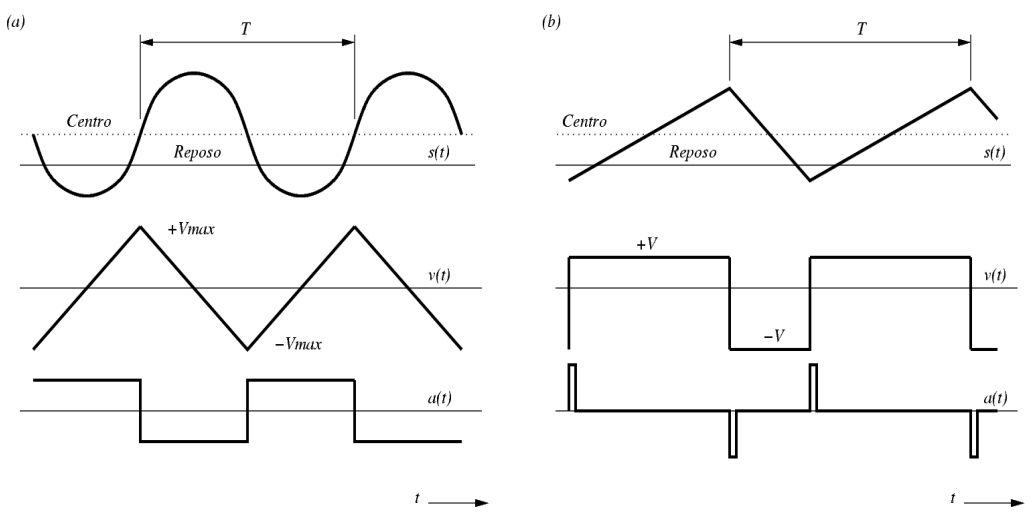

Figura 44: Formas de onda para el desplazamiento $s(t)$, la velocidad $v(t)$ y la aceleración a(t), en (a) modo aceleración constante y (b) modo velocidad constante.

Un valor medio no nulo en la referencia de velocidad no podrá ser seguido por el sistema, pues no tiene ganancia en continua. Sin embargo producirá un desplazamiento del eje que no puede ser detectado por la bobina sensora. Esto produce un cambio en la distancia media entre la fuente y el detector, modificando la tasa de trabajo. Los efectos de este fenómeno sobre el espectro no pueden diferenciarse de la absorción resonante, resultando en un error de medida. El offset de los amplificadores de salida del generador de referencia tiene dicho efecto. Aunque se anule dicho offset por procedimientos eléctricos, la deriva térmica (drift) del mismo produce efectos a largo plazo.

Una forma de reducir la sensibilidad al offset es tensando los resortes de suspensión, a expensas de modificar la frecuencia de resonancia, por lo que se requerirá de más potencia para accionar el transductor, además de la circulación de una corriente continua por la bobina que tiene baja resistencia dinámica. Entonces existe un compromiso entre la insensibilidad al offset y la ganancia del sistema. En términos del control esto implica que a lazo cerrado con realimentación unitaria, esquema presentado en la Figura 45, el sistema derivador no es controlable en continua.

Considerando la simplificación de la ecuación 35, puede calcularse la transferencia del sistema a lazo cerrado y la expresión del error:

$$
\begin{aligned}
& T(s)=\frac{s A(s)}{1+s A(s)} \\
& E(s)=\frac{1}{1+s A(s)}
\end{aligned}
$$




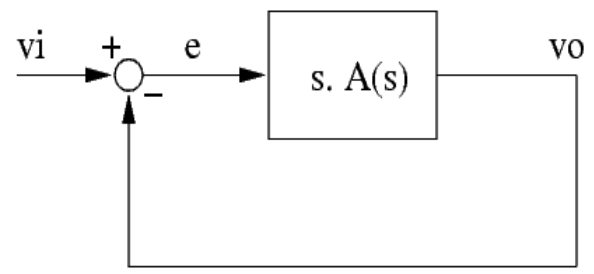

Figura 45: Sistema a lazo cerrado con realimentación unitaria

Puede verse que cuando la frecuencia de operación tiende a cero la función de transferencia se anula y el error tiende a uno, indicando que el sistema propuesto no es controlable en continua. A bajas frecuencias la ganancia de lazo cerrado es tan baja que las perturbaciones no pueden ser canceladas, por lo que la respuesta del sistema será la natural. La única forma de que este sistema sea útil es utilizando un montaje cuidadoso, libre de perturbaciones, sobre todo de baja frecuencia. Un montaje rígido a una masa muy grande permite obtener buenos resultados. En estas condiciones el arranque producirá un transitorio que será amortiguado por los resortes de suspensión. Una vez que éste se extinga el funcionamiento es aceptable si el transductor se mantiene libre de perturbaciones. Un cambio abrupto de la referencia producirá un transitorio equivalente al de arranque. Esto es crítico para el modo de funcionamiento a velocidad programable, pues constituye la esencia de su funcionamiento.

Como conclusión puede afirmarse que la característica derivadora a bajas frecuencias es contraproducente para el nuevo modo de funcionamiento deseado, principalmente porque la medición a velocidad constante se realiza en la zona plana de la referencia de velocidad. Aumentar el rendimiento del instrumento significa aumentar dicha zona de medición y por lo tanto aumentar el contenido armónico de bajas frecuencias. Sin embargo debe asumirse este comportamiento como una característica inevitable de los transductores electromecánicos que se desean utilizar. En lo que sigue de este capítulo se intentará optimizar el funcionamiento dentro del marco desfavorable descripto.

\subsection{El control de velocidad}

Como punto de partida se presenta el procedimiento de diseño de un sistema de lazo cerrado según las técnicas tradicionales, poniendo especial énfasis en las consecuencias de cada parámetro sobre el funcionamiento a velocidad programable. Si bien desde el inicio se apunta a un esquema realimentado con compensador serie y feedforward, se buscará optimizar el comportamiento del mismo para adecuarlo al nuevo modo de funcionamiento.

El procedimiento de diseño se presenta según los siguientes pasos: modelado, diseño de un control de lazo cerrado con compensador serie, agregado de un compensador feedforward para mejorar la respuesta a bajas frecuencias, simulaciones, y finalmente detalles acerca de la implementación.

\subsubsection{Modelado del sistema}

La función de transferencia de los transductores ha sido estudiada en detalle [Kankeleit 1964] [Cranshaw 1974]. En la Figura 46 puede verse una función de transferencia típica de este tipo 
de sistemas.

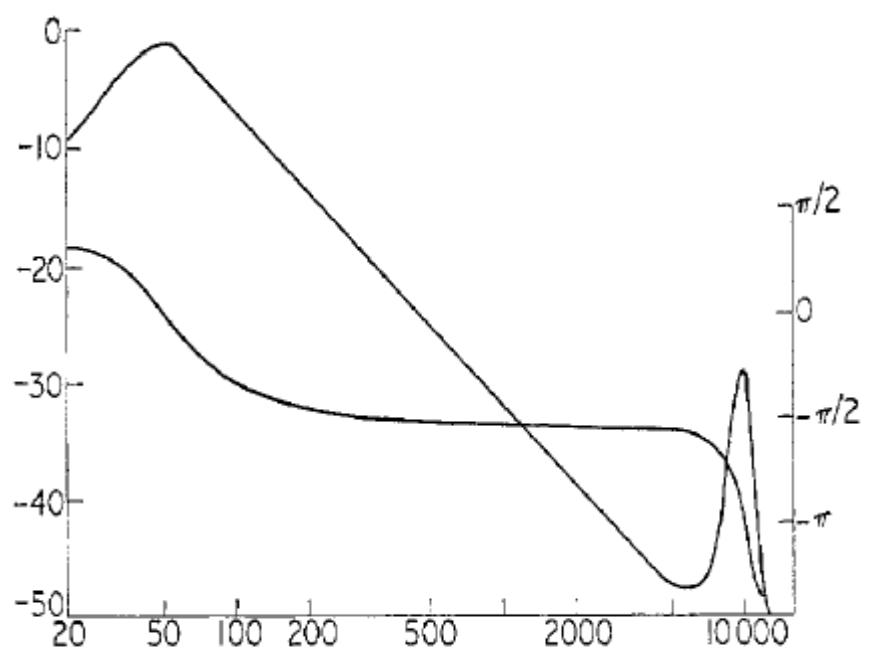

Figura 46: Función de transferencia de un transductor electromecánico [Cranshaw 1974]. En este caso la frecuencia natural se encuentra alrededor de los $40 \mathrm{~Hz}(Q=3)$ y la resonancia a altas frecuencias aparece a los $9 \mathrm{KHz}(Q=10)$.

En la zona de bajas frecuencias la característica es básicamente la de un derivador, como se mencionó anteriormente. Entre 10 y $100 \mathrm{~Hz}$ (según las características constructivas del dispositivo) la masa móvil forma un sistema resonante con los resortes de suspensión, presentando un comportamiento de segundo orden amortiguado. La transferencia alcanza un máximo a dicha frecuencia, a la que denominaremos la frecuencia natural del sistema. Si el mecanismo de suspensión es adecuado y se evitan los rozamientos, en este punto la ganancia del sistema es próxima a los $0 \mathrm{~dB}$. Por consiguiente, se requerirá la menor cantidad de potencia para la operación (máxima ganancia del conjunto) si se utiliza una referencia de velocidad con contenido armónico acotado y con su frecuencia fundamental coincidente con la frecuencia natural del transductor. Una referencia de velocidad sinusoidal tendrá el menor contenido armónico, pero resulta inconveniente por motivos que se presentaron previamente. Con una referencia triangular como la utilizada en experiencias de aceleración constante se obtienen un buenos resultados si la frecuencia fundamental de trabajo y la tercer armónica (dominantes en la triangular) se colocan en la zona de mayor ganancia. En el caso de una referencia cuadrada (que tiene un espectro menos acotado) la situación es más complicada. Este tema se explora en profundidad más adelante en este capítulo.

A altas frecuencias (entre 1 y $15 \mathrm{KHz}$ ) aparece una resonancia debida a la masa del conjunto, produciendo la inversión de fase. Este efecto (tan indeseable como inevitable) limita seriamente la ganancia proporcional aplicable al lazo de control. La técnica de compensación más adecuada en estos casos es la utilización de un compensador polo-cero que disminuya la ganancia a altas frecuencias, aumentando el margen de ganancia (ver a continuación). Un efecto indeseable de esta técnica es que el sistema resultante no presenta buena respuesta a referencias con alto contenido armónico, lo que es lo mismo que decir que no acepta cambios bruscos de velocidad (baja ganancia a altas frecuencias). De todos modos el ancho de banda del sistema debe mantenerse lo suficientemente amplio como para ser capaz de cancelar 
perturbaciones externas como ondas de sonido o pequeñas fricciones.

Dependiendo de múltiples aspectos mecánicos (sobre todo partes flojas) pueden aparecer resonancias adicionales, como por ejemplo si el cuerpo del transductor no está rígidamente sujeto a la mesa (cuerpo y mesa resuenan por separado).

Las transferencias a lazo abierto de los transductores disponibles fueron relevadas en laboratorio, utilizando las condiciones de funcionamiento, obteniéndose resultados similares al de la figura. Por ejemplo, el transductor ASA tiene la resonancia de baja frecuencia $p_{L}$ en $12 \mathrm{~Hz}$ con un amortiguamiento $\xi_{L}$ de 0.1 y la de alta frecuencia $p_{H}$ en $9000 \mathrm{~Hz}$ con un $\xi_{H}$ de 0.05. El transductor CMTE tiene las resonancias en 15 y $11500 \mathrm{~Hz}$, respectivamente. Los transductores presentan características similares, por lo que la estrategia de control será similar en ambos casos.

En el resto del capítulo se utilizará el siguiente modelo del transductor para las simulaciones, compuesto por el derivador y las dos resonancias:

$$
T(s)=\frac{K s}{\left(\left(\frac{s}{p_{L}}\right)^{2}+2 \xi_{L} \frac{s}{p_{L}}+1\right)\left(\left(\frac{s}{p_{H}}\right)^{2}+2 \xi_{H} \frac{s}{p_{H}}+1\right)}
$$

El efecto de la inductancia eléctrica de la bobina de accionamiento no está considerado en el modelo anterior, ya que puede manipularse para hacer sus efectos despreciables. Si se considera al bobinado como una resistencia $R_{D}$ en serie con una inductancia $L_{D}$, resulta que la corriente que circulará al aplicarse una tensión $v d$ de accionamiento será:

$$
i=\frac{v d}{\left(R_{D}+s L_{D}\right)}
$$

Esta corriente es la que produce la fuerza que desplaza al eje, por lo tanto en la transferencia se manifiesta como un polo en $w_{e}=R_{D} / L_{D}$. Los valores de resistencia e inductancia de los bobinados de accionamiento de los transductores disponibles se muestran en la Tabla 2.

\begin{tabular}{|l|c|c|}
\hline & ASA & CMTE \\
\hline $\mathrm{R}_{\text {DRIVE [ohms }]}$ & 20 & 60 \\
\hline $\mathrm{L}_{\text {DRIVE [mHy] }}$ & 22 & 50 \\
\hline$f_{e[H z]}$ & 150 & 190 \\
\hline
\end{tabular}

Tabla 2: Parámetros de los transductores disponibles en el laboratorio.

Una forma simple de minimizar los efectos, si se dispone de tensión suficiente a la salida del amplificador de potencia, es utilizar una resistencia en serie con el bobinado, lo cual desplaza el polo hacia la zona de altas frecuencias. Con este método se pone en fase la corriente, pero es necesario compensar la caída de tensión en la resistencia con más tensión de salida del 
amplificador. En el punto siguiente se muestra como puede implementarse un control proporcional para explotar al máximo esta compensación.

\subsubsection{Control proporcional con compensación serie}

El esquema de realimentación con ganancia proporcional de la Figura 47 puede implementarse como muestra la Figura 48 con un amplificador operacional como ganancia de tensión y un par complementario para la ganancia de corriente. La resistencia en serie con el bobinado se utiliza para regular la ganancia. La compensación del efecto de cruce por cero del par complementario es realizada en este caso por la realimentación, pudiéndose utilizar alguna técnica de compensación adicional si fuera necesario.

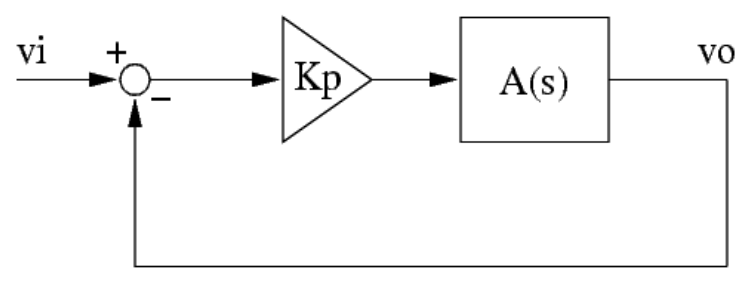

Figura 47: Control proporcional

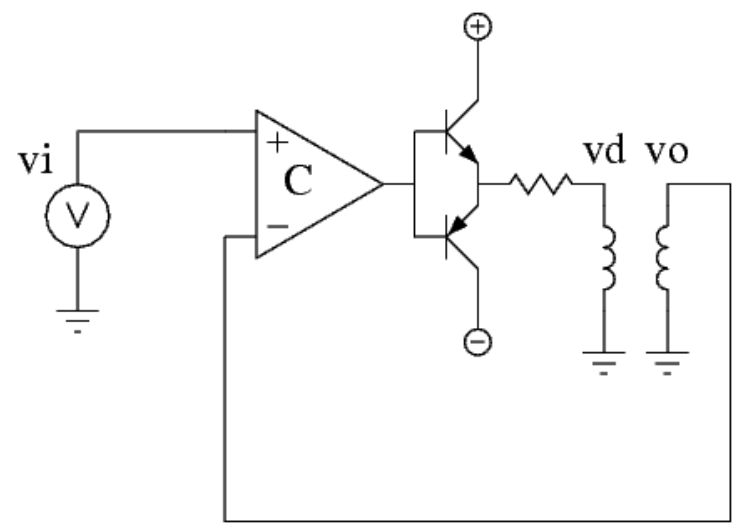

Figura 48: Implementación circuital del control proporcional.

Al aumentar la resistencia se produce un doble efecto de estabilización: disminuye la ganancia y aumenta la frecuencia del polo eléctrico. El menor error en la velocidad se producirá con el menor valor de resistencia que mantenga estable al sistema.

Sin embargo, el margen de ganancia del conjunto resulta menor que 150, lo cual no es suficiente para conseguir un error apropiado. Agregando un compensador serie como muestra la Figura 49 puede mejorarse dicho margen. Dado que la distancia entre las resonancias de baja y alta frecuencia es de casi tres décadas, puede utilizarse un compensador polo-cero como muestra la Figura 50 para aumentar en $20 \mathrm{~dB}$ el margen de ganancia. 


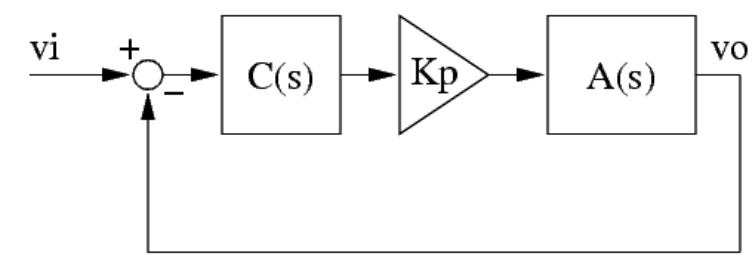

Figura 49: Control proporcional con compensador serie

Suponiendo que las resonancias se encuentran en 10 y $10000 \mathrm{~Hz}$ aproximadamente, puede colocarse el polo del compensador alrededor de los $100 \mathrm{~Hz}$ y el cero en $1000 \mathrm{~Hz}$. El margen de ganancia aumenta de 150 a 1500 (ver Figura 50), lo que permite una performance muy superior respecto del error. En caso de ser necesario puede correrse aún más el polo hacia las bajas frecuencias, lo que permite aumentar el margen de ganancia a expensas de deformar la zona de mayor ganancia.

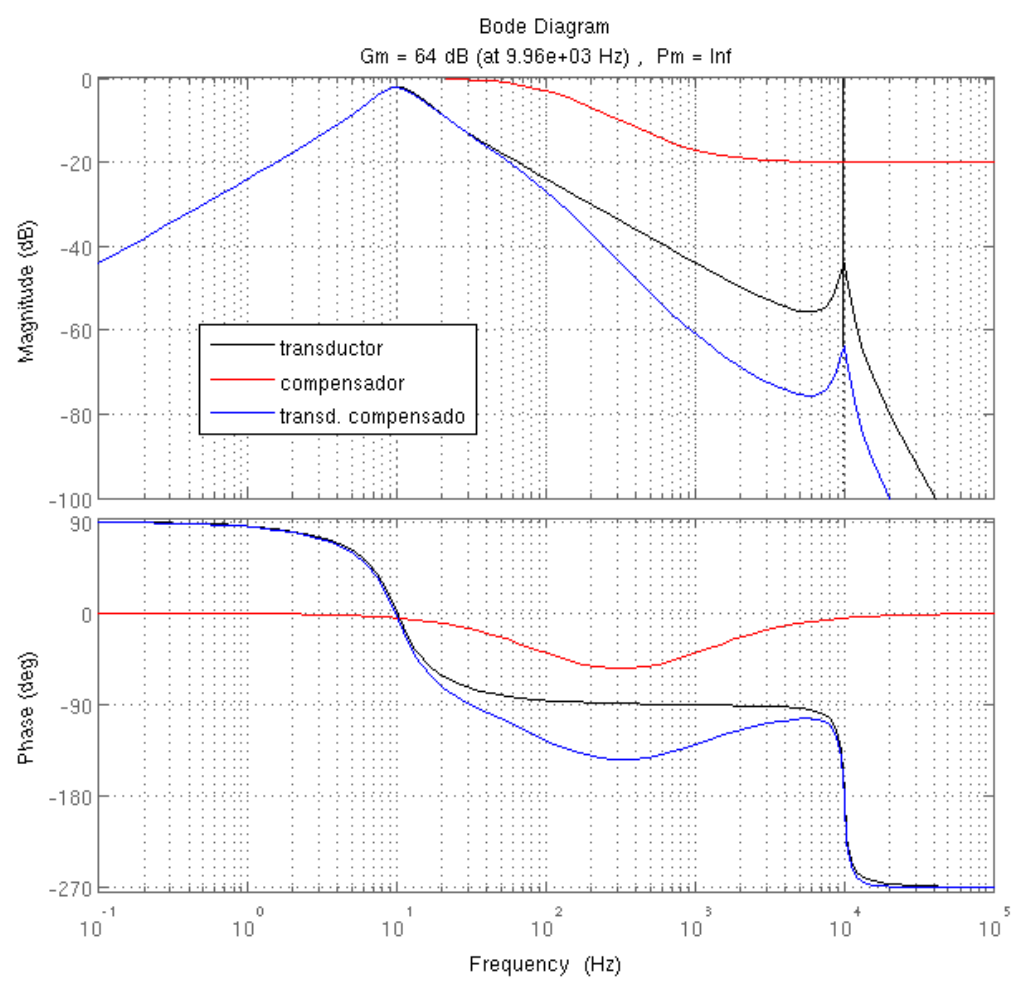

Figura 50: Diagrama de Bode del lazo con y sin compensador.

La utilización del compensador tiene algunas consecuencias negativas respecto del funcionamiento deseado. Si se reduce mucho el ancho de banda para aumentar el margen de ganancia, el sistema solo será sensible a un rango limitado de frecuencias. En estas condiciones el eje puede vibrar a altas frecuencias sin que el sistema de control lo detecte (por ejemplo vibraciones transmitidas por el piso). Para la operación a velocidad programable la reducción del ancho de banda puede ser crítica, pues los cambios abruptos de la referencia de velocidad son la base del funcionamiento. La baja ganancia a bajas frecuencias resulta en que, ante una perturbación o un cambio de referencia, pueden presentarse transitorios largos con 
oscilaciones de baja frecuencia que sólo serán amortiguadas por los resortes de suspensión.

A pesar de las limitaciones, este esquema de control tiene una buena performance para la técnica de aceleración constante. Los detalles acerca de la implementación circuital de este compensador se presentan, junto con el resto del sistema de control, en el Apéndice C.

\subsubsection{Compensación feedforward}

La técnica de control de velocidad presentada utiliza el esquema clásico de realimentación que se basa en la idea de la compensación del error. En este tipo de sistemas el error debe producirse para que la acción de control se genere. Un compensador de este tipo funciona atrasado, causando problemas de estabilidad si se desea operar con poco error. Si se dispone de información acerca del comportamiento del sistema a controlar, como en este caso, no es necesario esperar a que se produzca el error para actuar en consecuencia. Se puede actuar con un control directo (o de lazo abierto) frente al modo de funcionamiento predecible y se reserva la realimentación para el rechazo de perturbaciones impredecibles. A esta técnica se la conoce como feedforward (por oposición a feedback). Ambas técnicas pueden utilizarse combinadas para producir buenos resultados en el caso en estudio.

La Figura 51 presenta los esquemas de control en ambos casos, siendo $A$ el sistema a controlar, $C$ el compensador del lazo realimentado y $F$ el compensador feedforward.
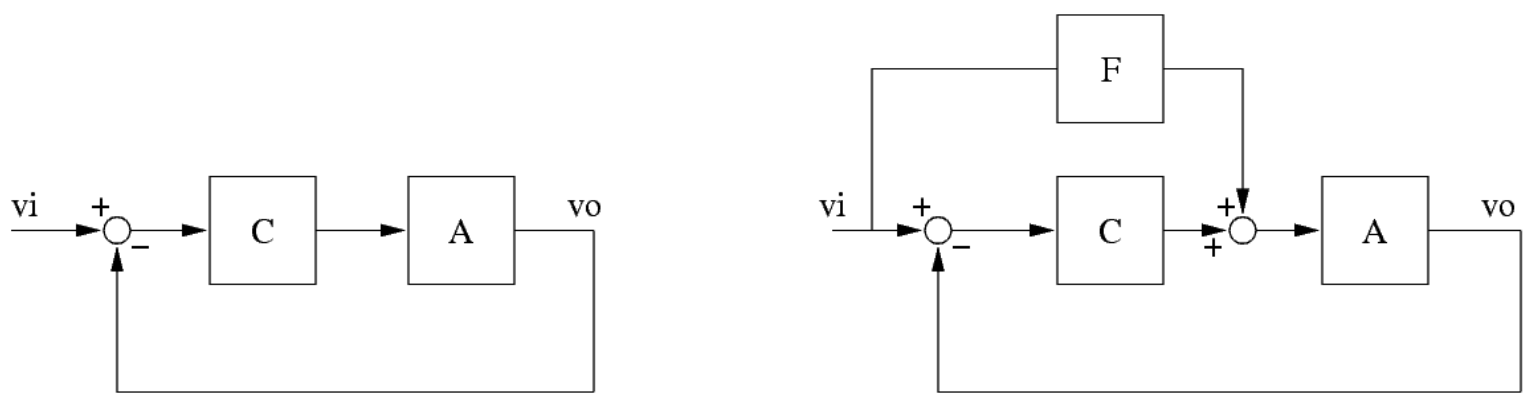

Figura 51: Esquemas a) realimentado y b) realimentado con feedforward.

El compensador feedforward no está en el lazo del sistema, por lo que no afecta las raíces de la ecuación característica del sistema original. Los polos y ceros del compensador pueden elegirse para añadir o cancelar los polos y ceros de la función de transferencia a lazo cerrado.

Para el esquema realimentado de la figura, la función de transferencia de lazo cerrado resulta:

$$
T_{R}=\frac{v o}{v i}=\frac{1}{1+\frac{1}{C A}}
$$

Para el esquema con feedforward la transferencia puede expresarse como: 


$$
T_{F}=\frac{v o}{v i}=\frac{1+\frac{F}{C}}{1+\frac{1}{C A}}
$$

$T_{F}$ puede hacerse unitario de dos maneras:

a) $\mathrm{Si}$ el compensador de realimentación $C$ tiene una ganancia suficientemente grande como para que el error se anule (esquema clásico de realimentación).

b) Si el compensador feedforward $F(s)$ es exactamente la inversa de la transferencia del sistema a controlar: $F(s)=1 / A(s)$ (control de lazo abierto).

Para el último caso, si $A_{0}$ es la función de transferencia nominal del sistema, puede utilizarse un bloque de feedforward tal que:

$$
F=\frac{1}{A_{0}}
$$

Resulta entonces que la transferencia de lazo cerrado del sistema con feedforward puede expresarse como :

$$
T_{F}=\frac{T}{T_{0}} \approx 1
$$

Para el caso de interés esta técnica es de implementación factible [Lurie 2000, p.33], pues:

i. El sistema a controlar es bien conocido: a bajas frecuencias se comporta como un derivador.

ii. El grado del numerador del bloque de feedforward es menor que el del denominador. Como se desea compensar la característica derivadora del transductor puede utilizarse como compensador feedforward un integrador, que cumple con esta condición.

iii. El sistema no tiene retardos puros.

Para el diseño puede suponerse que a bajas frecuencias el sistema a controlar es un derivador puro, por lo que su transferencia nominal será $A_{0}(s)=s A_{0}$. En lazo cerrado este sistema tiene un cero en el origen y un polo en $1 / C A$. La ganancia en continua es nula.

Si se agrega un compensador feedforward integrador con ganancia tal que $F(s)=F / s$, a lazo cerrado el cero se desplaza del origen a $F / C$, mientras que el polo permanece en $1 / C A$. La nueva ganancia en continua resulta igual a $F A$. Si $F=1 / A_{0}$, el polo y el cero se cancelan y la ganancia en continua vale $A / A_{0}=1$.

En resumen, utilizando el conocimiento previo disponible del sistema a controlar (pues se sabe que a bajas frecuencias se comporta como derivador) se actúa sobre el mismo utilizando dicha información a través del compensador $F$ a lazo abierto, que provee la integral de la referencia de velocidad (posición). El bloque $C$ del lazo de realimentación se encarga de compensar las perturbaciones y las diferencias entre el sistema real y el nominal. 
Debe considerarse que en la transferencia nominal del sistema no fueron tenidas en cuenta las resonancias del transductor a altas frecuencias. La compensación de las mismas estará a cargo del bloque $C$. Para esta tarea puede utilizarse un compensador similar al de la sección anterior. La rama de feedforward se utiliza en este caso sólo para mejorar la respuesta a bajas frecuencias del sistema.

Debe tenerse en cuenta que con la compensación feedforward reaparece un problema descripto anteriormente: al tener ganancia en continua, el valor medio de la referencia se realimenta, produciendo un efecto acumulativo en la posición. Por ello el valor medio de la referencia debe ser eliminado por completo. Cualquier método eléctrico que utilice a tal fin (acoplamiento en alterna, realimentar valor medio, etc.) vuelve a desmejorar la respuesta en bajas frecuencias, presentándose un compromiso difícil de equilibrar.

A fin de sintonizar el funcionamiento del compensador se utilizó el entorno Simulink de Matlab, basando el funcionamiento en el modelo anteriormente presentado. En la Figura 52 puede verse el esquema utilizado.

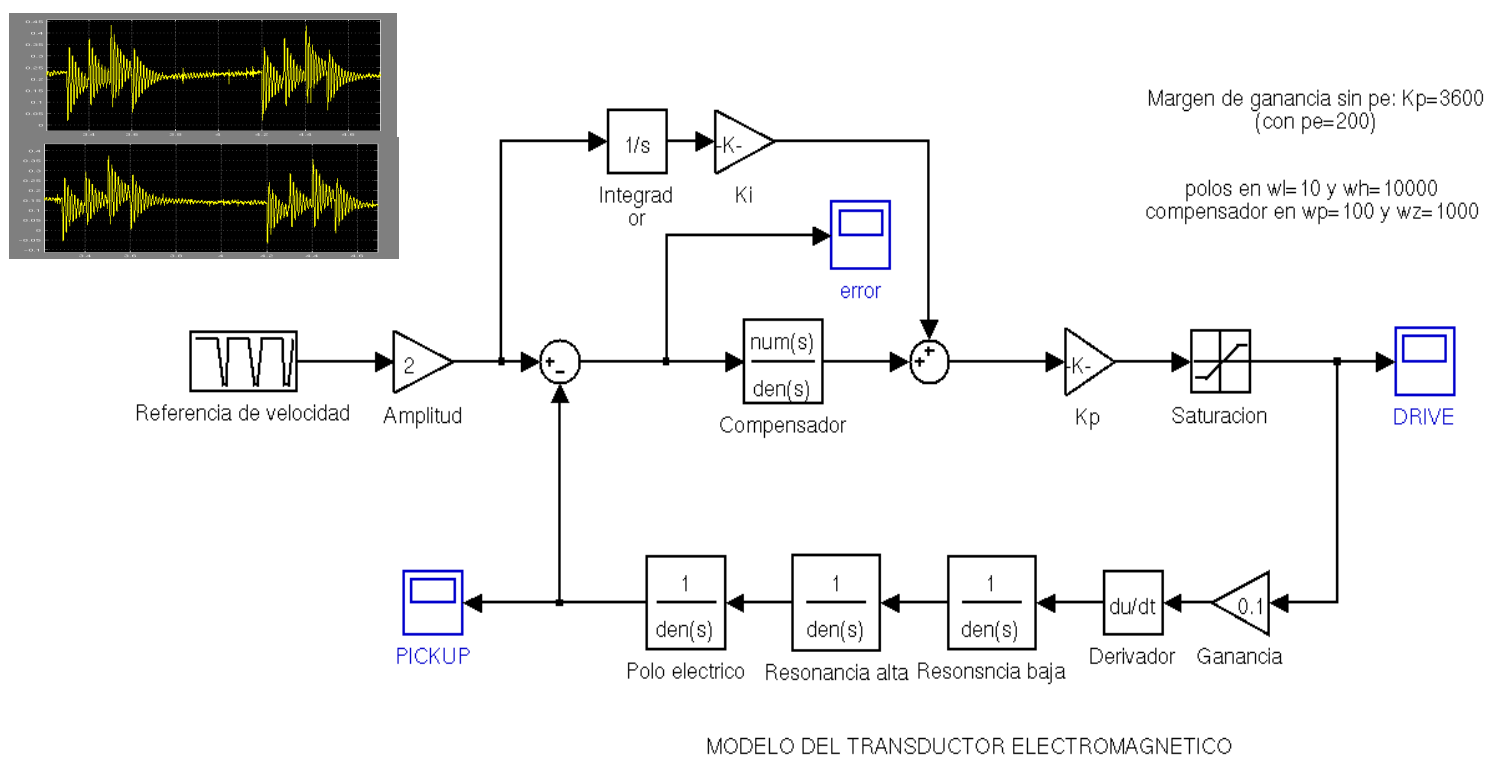

Figura 52: Modelo Simulink para el compensador.

Por este método pueden ajustarse el polo y el cero del compensador, la ganancia aproximada del lazo de feedforward y la ganancia proporcional máxima. Es interesante ver como la ganancia del feedforward ajusta la pendiente del error (insert de la figura). La implementación práctica del circuito debe contemplar un ajuste manual de dichos parámetros, a fin de que puedan ser ajustados para minimizar el error en cada condición particular de trabajo.

\subsubsection{Análisis de performance}

Con los ajustes basados en simulaciones puede obtenerse una buena performance a velocidad constante. Sin embargo la sintonización del sistema debe ser corregida si se modifica el contenido armónico de la referencia. Esto hace que esta estrategia de control sea útil sólo para 
los espectrómetros en los cuales todo el experimento se realiza con una única referencia de velocidad.

El panorama no es muy alentador si se desea controlar el transductor con una referencia cuadrada de diferentes amplitudes (velocidad programable). La mala respuesta en bajas frecuencias provoca una respuesta pobre ante la zona útil de medida a velocidad constante. Por otro lado, la limitación del ancho de banda del sistema en altas frecuencias, necesaria para ampliar el margen de ganancia, introduce respuestas lentas ante los cambios abruptos de la referencia. Estos son necesarios para hacer retroceder el eje rápidamente y colocarlo nuevamente a la velocidad de interés.

La forma de retorno trapezoidal ayuda a reducir el transitorio ya que elimina los escalones de velocidad que requerirían potencia infinita, a expensas de prolongar el tiempo de retorno.

En general puede afirmarse que el sistema de control descripto hasta el momento es adecuado para la técnica de aceleración constante, que debe utilizarse con precaución a velocidad constante, y que puede ser utilizado para la técnica de velocidad programable sólo si el rango de variación de la velocidad es mínimo. Sin embargo es un buen punto de partida a partir del cual introducir algunas mejoras.

A continuación se presentan dos mecanismos que, combinados con el esquema de control presentado, permiten mejorar las respuestas del sistema en baja y alta frecuencia, a fin de adecuarlas a los requerimientos de la operación a velocidad constante programable.

En el primero de ellos propone eliminar los mecanismos eléctricos de cancelación del offset de la referencia, reemplazándolos por una realimentación de posición de simple implementación con optoacopladores. Esto permite recuperar el buen funcionamiento en bajas frecuencias.

El mecanismo de mejora en altas frecuencias se centra en encontrar la forma de onda de retorno del eje que produzca la respuesta más adecuada del sistema. Este se complementa con un mecanismo de sincronización para los cambios de velocidad.

\subsection{Técnica de accionamiento con realimentación de posición}

En esta sección se presenta una primer solución al problema planteado de velocidad programable. Este involucra el sensado de una variable adicional, como es la posición, lo cual complica la configuración y el montaje del equipamiento de laboratorio. Sin embargo su impacto no es considerable y permite obtener buenos resultados experimentales. Se propone como una primer herramienta para hacer frente al problema.

\subsubsection{Fundamentos}

El principal inconveniente del sistema de control descripto se origina en que inevitablemente la referencia de velocidad presentará un valor de continua, dado al menos por el offset de los amplificadores de salida del generador de tensión. En el caso de realimentación de velocidad con compensador serie y ganancia proporcional, este valor medio producirá sólo un efecto de desplazamiento fuera del centro del recorrido, acercando o alejando la fuente radiactiva al detector. En este caso, sólo la variación en el tiempo de este desplazamiento (drift) produce 
un error en la velocidad del eje. Como se mostró, la sensibilidad a este efecto puede disminuirse tensando los resortes de suspensión, lo cual no es una solución adecuada más allá de cierto punto, ya que aumenta la frecuencia natural del sistema y disminuye su ganancia. Si para la implementación se utilizan amplificadores con bajo offset y drift, el desplazamiento del centro de la trayectoria puede reducirse y el error en velocidad será proporcional a la velocidad de variación del mismo, por lo que puede despreciarse durante el normal funcionamiento del espectrómetro. Es importante notar en este punto que, además de controlar la velocidad del eje, el sistema de control debe garantizar un valor medio constante de la misma, ya que este parámetro representa la distancia entre la fuente y el detector y por lo tanto la tasa de conteo media.

En el esquema de realimentación con feedforward la situación es más complicada. Al no ser nula la ganancia en continua, el offset de la referencia necesariamente implica un valor medio en la velocidad del eje. Esto produce un desplazamiento acumulativo en la posición (la rama feedforward integra el valor medio) que lo llevará a largo plazo fuera del rango de operación. El tensado de los resortes no soluciona este problema. Solo puede retrasar los efectos. Cualquier método eléctrico que se utilice para eliminar el valor de continua de la referencia volverá a desmejorar la ganancia en baja frecuencia del sistema.

En esta sección se propone eliminar el mecanismo eléctrico de cancelación del valor medio, reemplazándolo por un procedimiento mecánico/óptico simple. Se consigue así acoplar en continua la referencia de velocidad. El método se basa en que no es necesario disponer de la información de la posición como una variable continua. Es suficiente realimentar dos puntos y utilizarlos para mantener la trayectoria acotada y por lo tanto nulo el valor medio del desplazamiento. Por este procedimiento se elimina la componente de continua de la velocidad, sin afectar su funcionamiento a bajas frecuencias.

\subsubsection{Modos de operación}

Antes de continuar con el resto de las consideraciones, es importante hacer una diferencia entre dos posibles modos de operación a velocidad constante del transductor:

a) Operación a frecuencia constante: En este caso se mantiene constante la frecuencia de la referencia de velocidad. Al modificarse la velocidad de trabajo cambia la distancia recorrida, tal que si la velocidad aumenta, el recorrido aumenta.

b) Operación a desplazamiento constante: El recorrido del eje está acotado por dos puntos fijos en el espacio (fines de carrera). Al aumentar la velocidad del eje en la zona de medida aumenta la frecuencia de operación.

En la Figura 53 puede verse la evolución temporal de dos referencias para diferentes velocidades en ambos modos de operación. 

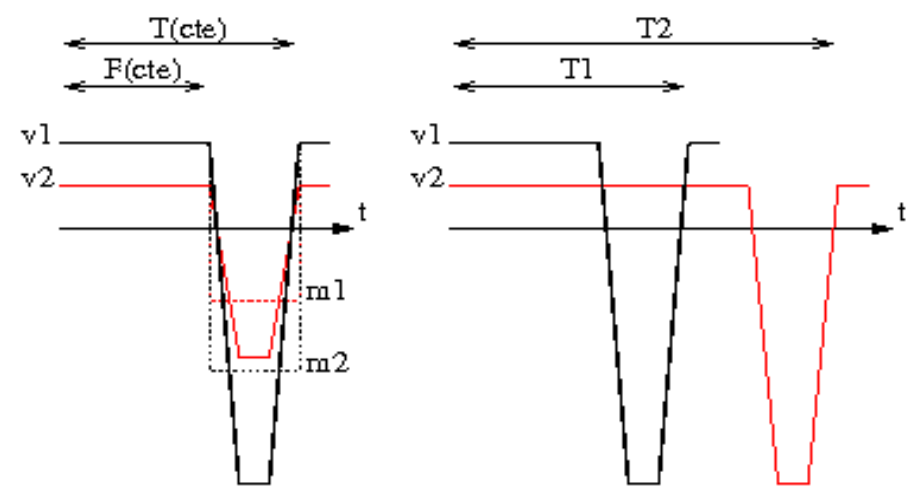

Figura 53: Tensión de referencia para dos velocidades distintas (tal que $v_{l}=2 v_{2}$ ), utilizando la estrategia de frecuencia constante (a) y de desplazamiento constante (b)

Ambos modos tienen ventajas y desventajas, en relación al funcionamiento a velocidad programable.

El segundo método no tiene el mismo ciclo de medida para todas las velocidades, ya que la duración de la zona plana es mayor cuanto menor sea la velocidad utilizada, mientras que el tiempo de retorno (a máxima velocidad) es igual para todas las velocidades. Esto puede ser un problema, pero aumenta notablemente el rendimiento a bajas velocidades. Esto lleva a que el contenido armónico sea variable, aumentando notablemente el contenido de bajas frecuencias para velocidades bajas.

En el primer caso la forma del espectro de frecuencias se mantiene constante para todas las velocidades, modificándose las amplitudes del mismo. Este modo tiene la desventaja de aumentar el recorrido para altas velocidades. Debe tenerse especial cuidado en que la magnitud del máximo recorrido sea despreciable frente a la distancia al detector, para evitar las consecuencias mencionadas del efecto de ángulo sólido. El método de desplazamiento constante no presenta este inconveniente, ya que su recorrido está acotado para todas la velocidades.

En el ejemplo de la figura puede verse cómo a frecuencia constante dos velocidades diferentes tienen la misma eficiencia, mientras que la más lenta tiene la mitad de desplazamiento. A desplazamiento constante las dos opciones recorren la misma distancia y ambas tardan lo mismo en volver, por lo tanto la más lenta tiene mayor eficiencia y mayor contenido armónico de bajas frecuencias.

Ambos modos de operación requieren distintas estrategias en el momento de implementar la realimentación discreta de posición.

En el caso de la operación a desplazamiento constante la realimentación de posición puede hacerse con dos sensores discretos colocados en los extremos del recorrido. Dichos fines de carrera pueden utilizarse para generar automáticamente la referencia, conmutando entre la velocidad de medida programable y la de retorno, fija para todas las velocidades en un valor óptimo. Para ello debe disponerse de una tensión continua proporcional a la velocidad deseada y una tensión que represente la máxima velocidad de retorno, la cual se aplicará en todos los casos. 
En el caso de la operación a frecuencia constante no pueden utilizarse fines de carrera pues el recorrido varía con la velocidad de operación. El único punto en el espacio común a todas las velocidades es el centro (punto de reposo), lo que lo convierte en la única opción de realimentación discreta. Dicho punto es transitado dos veces por ciclo, en diferentes direcciones. Por lo tanto la realimentación de posición es más complicada en este caso: se dispone de un solo punto en que debe detectarse la dirección y además debe disponerse de algún mecanismo de medición de tiempo para conmutar las referencias en el momento apropiado. Se descarta la operación con un punto en un extremo, pues las trayectorias no estarían centradas y la dinámica del sistema sería diferente para cada velocidad.

En ambos casos se elimina el problema del valor medio de la referencia de velocidad, al fijarse uno o dos puntos del espacio. Permanece el efecto de la variación del offset que se suma a la velocidad, pero que es despreciable pues en este caso no produce efectos acumulativos.

\subsubsection{Implementación}

Para la implementación del modo de operación a desplazamiento constante se utilizaron dos foto-interruptores, consistentes en un LED infrarrojo acoplado a un foto-transistor de silicio NPN en un encapsulado diseñado especialmente para la detección sin contacto en una ranura. La disposición se muestra en la Figura 54a. Estos sensores ópticos se montaron una distancia fija $R$ sobre una placa de circuito impreso, junto con la electrónica necesaria para el acondicionamiento de las dos señales. La placa se montó rígidamente al cuerpo del transductor. Para activar los interruptores se utilizó la sombra producida por una pieza delgada de aluminio de ancho $S$, montada solidaria al eje en el sentido del recorrido.

La distancia total recorrida por el eje resulta:

$$
2 \Delta=R-S
$$

Se calibraron varias sombras intercambiables de distinto ancho, para experimentar con diferentes recorridos. La masa agregada al eje resultó despreciable: dos tuercas y la pieza de aluminio que suman aproximadamente $10 \mathrm{~g} \mathrm{(5 \%} \mathrm{del} \mathrm{total} \mathrm{del} \mathrm{eje).}$

Este modo de operación presenta una limitación a bajas velocidades. Para cada velocidad medida debe completarse un número entero de recorridos, para que la distancia promedio entre la fuente y el detector sea la misma en todos los casos. El problema es que el tiempo necesario para realizar un recorrido completo puede ser muy largo para velocidades bajas. Por ejemplo, si $2 \Delta=2 \mathrm{~mm}$ la medición del canal correspondiente a $0.05 \mathrm{~mm} / \mathrm{s}$ toma al menos 40 segundos.

Esta estrategia se modificó como muestra la Figura 54b para experimentar la realimentación de posición en el caso de accionamiento a frecuencia constante. Se utilizó un elemento óptico diferencial de dos canales que permite determinar dirección del movimiento en un punto. Para la sombra se utilizó una varilla de aluminio de diámetro calibrado $(1.5 \mathrm{~mm})$. La rigidez del conjunto no resultó tan satisfactoria como en la implementación con fines de carrera, además de presentar la complicación adicional de utilizar temporizadores para calcular los cambios de referencia. 
Para la verificación experimental de la técnica de desplazamiento constante con fines de carrera se realizó un montaje de laboratorio consistente en una etapa de detección estándar, un transductor ASA modificado según la Figura 54a, más el control proporcional con compensador y feedforward acoplado en continua. Un sistema de adquisición basado en un microcontrolador fue diseñado a tal efecto. Las señales lógicas generadas por la realimentación de posición se utilizaron para conmutar las referencias de velocidad generadas con un conversor DA.
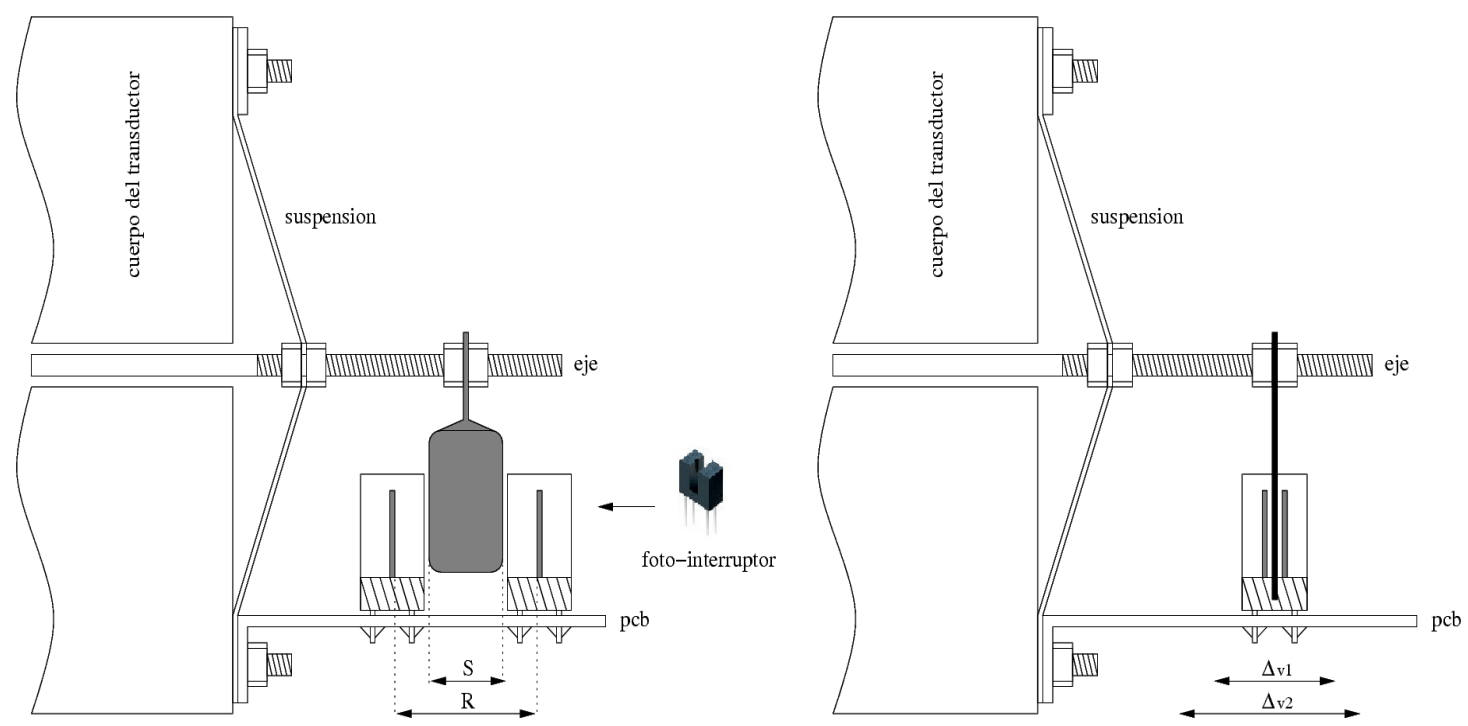

Figura 54: Implementación de la realimentación de posición para a) la técnica de desplazamiento constante y b) la técnica de frecuencia constante.

El procedimiento de calibración arrojó resultados muy favorables en lo que se refiere a la resolución y a la linealidad del sistema respecto de la velocidad de operación, consiguiéndose espectros de calidad comparable a los obtenidos con la técnica de aceleración constante. El ciclo de trabajo variable resultó en un rango desde de 0,6 para altas velocidades hasta casi 1 para bajas velocidades.

El sistema completo, incluyendo la implementación del ratímetro, se muestra en el Apéndice C, junto con los detalles y resultados de la calibración.

\subsection{Técnica de accionamiento suave (smooth)}

En esta sección se presentará un enfoque diferente respecto del accionamiento a velocidad constante de los transductores electromecánicos. Si bien hasta el momento las técnicas con realimentación de posición han permitido obtener resultados razonables, esto se ha logrado a expensas de realizar adaptaciones mecánicas en el transductor. Aunque éstas son de implementación simple y no requieren maquinado de precisión, es deseable obtener una solución alternativa que no implique intervención mecánica alguna.

A continuación se mostrará cómo una manipulación adecuada de la trayectoria del eje en el modo de frecuencia constante permite obtener mejoras considerables en el desempeño del sistema de control. 


\subsubsection{Fundamento}

En el accionamiento a frecuencia constante el contenido armónico de la referencia en bajas frecuencias se encuentra acotado, siendo su componente mínima la fundamental de la referencia. Esta no puede ser muy baja si se desea mantener restringido el recorrido. Este aspecto marca una importante diferencia en comparación con la técnica de desplazamiento constante, en la cual la frecuencia fundamental de operación tiende a cero cuando la velocidad disminuye. En el caso del accionamiento a frecuencia constante, el contenido armónico de la referencia es idéntico para todo el rango de velocidad. El cambio de la velocidad de operación se consigue modificando la amplitud de la referencia, como mostró la Figura 53.

Este aspecto es importante si se tiene en cuenta la función de transferencia del transductor de la Figura 46. Esta posee una zona de muy baja atenuación alrededor de la frecuencia de oscilación natural, aumentando la misma muy rápidamente hacia las altas y bajas frecuencias. Teniendo en cuenta el limitado margen de ganancia del lazo, a consecuencia de la resonancia presente a alta frecuencia, y considerando la inevitable limitación en la acción de control, dadas las características de la bobina de accionamiento, resulta indispensable operar al equipo en las inmediaciones de la resonancia. Sería entonces deseable utilizar una referencia de velocidad con un contenido armónico limitado a la zona de mayor ganancia del transductor. Dicha referencia debe mantenerse acotada para todas las velocidades de trabajo, lo cual descarta la operación a desplazamiento constante.

Pero además de operar el dispositivo a frecuencia constante (lo que garantiza la mínima frecuencia de operación) debe ponerse también especial atención en el contenido armónico en altas frecuencias de la referencia. Este estará impuesto por el ciclo de trabajo (cuanto menos tiempo tome el regreso mayor será el contenido en altas frecuencias) pero también se verá afectado por la forma de la referencia de velocidad en esta zona. Es importante notar que si bien la zona plana está impuesta por el funcionamiento, la forma del retorno puede ser elegida a conveniencia, mientras conserve el valor medio adecuado.

La Figura 55 muestra algunas posibles referencias [Veiga 2006]. Puede verse cómo para un determinado período de operación $T$ se dispone de la opción de sacrificar tiempo de medida $F$ con el objetivo suavizar el movimiento, utilizando la misma velocidad máxima de retorno (rango dinámico de la referencia) para todos los casos. El ciclo de trabajo del sistema se define por la relación $d=F / T$.

La mejora de la opción trapezoidal $v_{2}$ respecto de la rectangular $v_{1}$ ya se mencionó en el Capítulo 2. En la opción rectangular la presencia de aceleración impulsiva condena necesariamente al sistema a la saturación de la acción de control. Un retorno sinusoidal como $v_{3}$ evita las discontinuidades en la aceleración eliminando la necesidad de variaciones abruptas en la fuerza proporcionada por el campo magnético. Incluso una forma más elaborada como $v_{4}$ suaviza aún más la aceleración, ya que la derivada de la aceleración será continua, a expensas de un valor mayor de la aceleración máxima. 

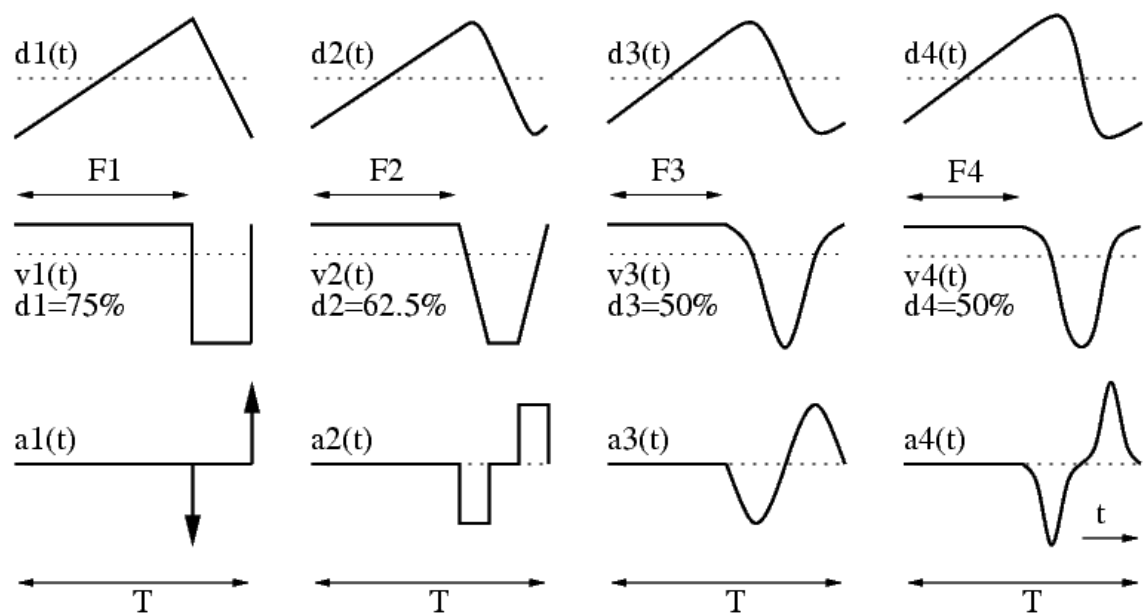

Figura 55: Desplazamiento $d(t)$, velocidad $v(t)$ y aceleración a $(t)$ para cuatro formas de onda de velocidad con contenido armónico descendente a expensas del tiempo neto de medida.

La proporción entre el tiempo útil de medida y el período del instrumento no sólo depende del ciclo de trabajo de la referencia. Debe tenerse en cuenta también el tiempo de establecimiento de la velocidad del eje. El conteo debe ser inhabilitado no sólo durante el retorno sino también durante un tiempo adicional en el comienzo de la zona plana de medida, como se mostró en la Sección 2.4. Este tiempo (GATE) debe ser programable por el usuario a fin se que resulte lo más ajustado posible a la respuesta transitoria del sistema particular. La Figura 56 muestra el tiempo efectivo de conteo $F^{\prime}$ que resulta en el caso práctico de una referencia abrupta. En este contexto, el ciclo de trabajo efectivo debe calcularse como $d^{\prime}=F^{\prime} /$ $T$.

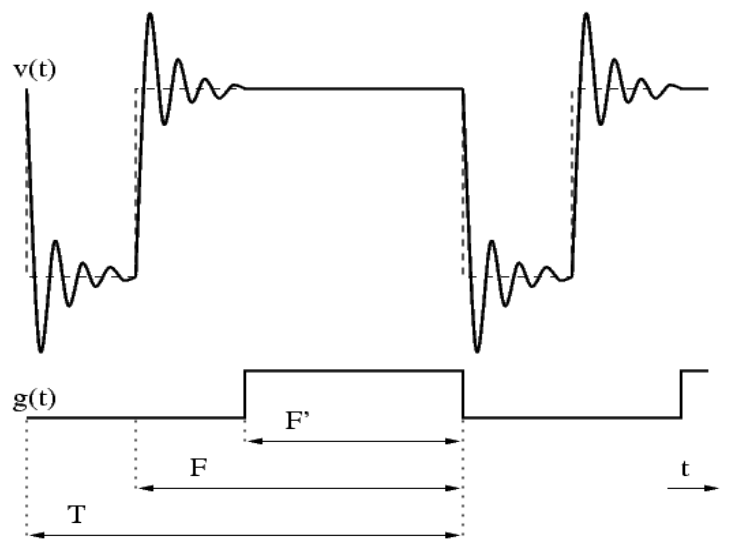

Figura 56: Calibración de la señal de habilitación de conteo en el caso de respuesta transitoria subamortiguada del transductor (línea llena) ante una referencia cuadrada de velocidad (línea punteada). Nótese la importante reducción del ciclo de trabajo efectivo, de 75 a $50 \%$.

El tiempo de establecimiento $F-F^{\prime}$ será mayor cuando se utilicen referencias más abruptas, o sea con mayor contenido armónico. Las formas más suaves insumen mayor tiempo en el 
retorno pero podrían presentar transitorios más cortos o incluso nulos. En lo que resta de esta sección se intentará encontrar la forma de la referencia que proporcione un mayor aprovechamiento de los recursos, o sea que redunde en un mayor ciclo de trabajo efectivo.

\subsubsection{Especificación de la referencia}

Se requiere entonces una referencia que, además de cumplir con la zona plana de velocidad constante, cumpla con las siguientes condiciones:

i. Debe ser periódica, ya que el recorrido del eje está limitado.

ii. Su valor medio debe ser nulo, ya que se trata de una referencia de velocidad.

iii. La zona plana debe ser lo más grande posible en relación al tiempo de retorno, para mejorar el ciclo de trabajo.

iv. La trayectoria de retorno debe exigir lo menos posible al sistema de control, a fin de requerir menor acción de control. Esto implica que la referencia de velocidad debe presentar un contenido armónico reducido para que pueda ser ubicado en las inmediaciones de la zona de mayor ganancia.

Los puntos i y ii no presentan muchas opciones respecto del diseño por ser evidentes, pero entre iii y iv se plantea un compromiso interesante que se analizará a continuación.

La transferencia del transductor tiene una resonancia en coincidencia con la inversión de fase, lo que resulta en un reducido margen de ganancia. Se pudo obtener una mejora utilizando un compensador, a expensas de perder ganancia en altas frecuencias. Por lo tanto la ganancia del sistema de lazo cerrado será muy baja fuera de las inmediaciones de la frecuencia natural de oscilación. El error del control proporcional se verá reducido si se puede garantizar una referencia de velocidad con contenido armónico acotado a dicha zona de alta ganancia.

Es interesante analizar este mismo problema desde el punto de vista mecánico. Intuitivamente, puede decirse que será más fácil mover un objeto si se le pide una trayectoria "suave", lo más parecida posible a su movimiento natural. Analizando el movimiento del eje en mayor detalle resulta que:

i. La variable a controlar es la velocidad del eje $v$. La trayectoria durante el retorno es el parámetro a optimizar. Puede ser cualquiera que tenga el mismo valor medio que la zona plana del tiempo de medida, para que el eje regrese al mismo lugar del que partió.

ii. La aceleración obtenida $a$ es la derivada primera de la velocidad del eje $v^{\prime}$. En la zona de velocidad constante la aceleración será nula y durante el retorno tendrá una forma que dependerá del retorno utilizado.

iii. La fuerza $\mathbf{F}_{\mathbf{T}}$ que debe aplicarse al eje es proporcional a la aceleración que se desea obtener. Por la segunda ley de Newton $\mathbf{F}_{\mathrm{T}}=$ m.a, siendo $\mathbf{F}_{\mathrm{T}}=\mathbf{F}_{\mathrm{B}}-\mathbf{F}_{\text {nat }} \cdot \mathbf{F}_{\text {nat }}$ es la fuerza asociada al movimiento armónico simple, ejercida por el sistema de suspensión y $\mathbf{F}_{\mathbf{B}}$ es la fuerza que ejerce el campo magnético.

iv. La fuerza $\mathbf{F}_{\mathbf{B}}$ se obtiene sobre un conductor que conduce una corriente $i$ dentro de un campo magnético $\mathbf{B}$ en la dirección perpendicular $\mathbf{d x}$, según $\mathbf{F}_{\mathbf{B}}=\mathrm{i}(\mathbf{d x} \mathrm{X} \mathbf{B})$. En este 
caso los vectores están dispuestos en una geometría ortogonal, por lo que $F_{B}=B i$. Por lo tanto $i$ debe tener la forma de $F_{B}$, o sea de $\left(a-a_{n a t}\right)$.

v. La corriente de accionamiento $i$ se obtiene aplicando una tensión $V$ a un bobinado de inductancia $L$, según $V=L d i / d t$. De aquí resulta un límite en la derivada de la corriente, dado por $d i / d t<V / L$. Este efecto limita la fuerza que puede realizar el campo $(d F / d t<B V / L)$ y por lo tanto la aceleración $(d a / d t<B V / m L)$.

vi. Resulta que a debe ser continua para no requerir $V$ infinita, pero además es conveniente que sea derivable, para no requerir escalones de tensión con rise-time muy grande, ya que la acción de control de tensión $V$ es proporcional a la derivada segunda de la velocidad $v^{\prime \prime}$.

En resumen, es conveniente que la aceleración $\left(\mathrm{v}^{\prime}\right)$ sea continua y moderada, para no requerir fuerzas muy grandes o discontinuas (con saltos instantáneos), lo que implicaría corrientes discontinuas en el arrollamiento inductivo (lo que requeriría tensiones infinitas o muy grandes). Al mismo tiempo es importante que $\mathrm{v}^{\prime \prime}$ también sea continua y moderada, para no depender del slew-rate del amplificador de salida de la etapa de accionamiento.

Este criterio puede extenderse, ya que cualquier sistema real tiene una gran cantidad de órdenes. En principio podría afirmarse que es conveniente que la forma de onda de la velocidad tenga la mayor cantidad posible de derivadas continuas, y que dichas derivadas tengan valores acotados. Esta condición implica un recorrido suave (se utiliza en estos casos el término smooth). Smoothness es una medida de la suavidad de una función en el tiempo, siendo una medida de la cantidad de derivadas continuas que ésta tiene.

Este factor está directamente relacionado con el contenido armónico de la función. La propiedad de localización de la transformada de Fourier indica que la suavidad en el tiempo se traduce en estrechez de banda en la frecuencia o decay. Por lo tanto si $f(t)$ es varias veces diferenciable, entonces $F(\mathrm{w})$ decae rápidamente a cero cuando $w$ tiende a $\pm \infty$. Una forma de expandir en el tiempo (para conseguir compresión o decay en frecuencia) es utilizando una función $f(t)$ que sea diferenciable la mayor cantidad de veces posible (smooth). Esta función estará dispersa en $\mathrm{t}$, garantizando un buen decay en w.

Como regla general, dada una función $f(t)$ y su transformada $F(w)$, cuanto más dispersa se encuentre $f$ en el eje de tiempo, más localizada se encontrará $F$ en el eje $w$. En particular, si se concentra una función en $t$, su transformada se expande en $w$ y viceversa. No se pueden concentrar simultáneamente la función y su transformada.

Cuando una función iguala a su transformada se consigue un balance particular entre localización y expansión. Dichas funciones se denominan self-dual functions. El ejemplo más importante de este tipo de funciones lo componen los múltiplos de la función gaussiana $f(t)=\exp \left(-t^{2} / 2\right)$.

El compromiso entre la localización de una función y su transformada puede formalizarse. Suponiendo que $f(t)$ y $F(w)$ son un par transformado de una función de energía finita, puede demostrarse [Papoulis 1962, Cap. 4, pp. 62-74] [Bracewell 1965, Cap. 8, pp. 160-163] que:

$$
\sigma_{f}^{2} \sigma_{F}^{2} \geq \frac{1}{4 \pi}
$$


Donde $\sigma^{2}$ es la varianza de la función. La igualdad es válida para la gaussiana, por lo que puede decirse que está concentrada al máximo en tiempo-frecuencia ${ }^{6}$.

Queda así planteado un compromiso en el momento de seleccionar una forma para el retorno del eje. Si se desea maximizar el tiempo de medida a velocidad constante (zona plana) se debe reducir al mínimo el tiempo de retorno. Un retorno rápido del eje (concentración en $t$ ) implica necesariamente un aumento del contenido armónico del movimiento (expansión en $f$ ), el cual cae fuera del reducido rango de frecuencias que el sistema de lazo cerrado puede manejar con buena ganancia.

La ecuación 34 puede indicar un camino para seleccionar una referencia que además debe ser nula en los extremos (para que empalme con la zona plana) y tener valor medio no nulo (para compensar el valor medio de la zona plana).

\subsubsection{Selección de la forma de la referencia}

Para verificar las propiedades de las diferentes opciones se diseño el siguiente experimento. Fijando un ciclo de trabajo idéntico para todas las formas de onda a estudiar, además de un mismo valor medio, se calcularon por métodos numéricos las tres primeras derivadas (para verificar smoothness) y la transformada rápida de Fourier (para verificar decay) de las funciones periódicas en estudio. La mejor forma para la referencia será la que tenga un mejor balance entre las siguientes propiedades:

- gran concentración de la transformada de Fourier;

- reducida amplitud máxima en el retorno (rango dinámico);

- reducida amplitud en la derivada segunda (rango dinámico de la acción de control);

- reducida amplitud en la derivada tercera (slew-rate de la acción de control).

De la gran cantidad de opciones analizadas se presentarán sólo los casos más interesantes y sus características principales. En la Figura 57 puede verse una comparación temporal entre algunas de las formas estudiadas.

En una primera categoría pueden colocarse las funciones que se componen de varios tramos continuos empalmados para lograr un retorno del período deseado (las primeras de la figura). Los tramos se manipulan para unirse suavemente con la zona plana y para obtener el valor medio adecuado (la mitad del valor máximo en este caso). Dentro de esta categoría pueden mencionarse los siguientes tipos.

\section{a) Polinomiales}

La forma más simple de obtener obtener la forma deseada es utilizando polinomios de orden ascendente, como por ejemplo: rectangular en un tramo, llamada [Rectangular] en las figuras; triangular en dos tramos (o trapezoidal en tres) [Trapezoidal]; parabólica en tres tramos [Parábola]; e hiperbólica en dos tramos [spline]. Inicialmente se descartaron la forma de onda rectangular y trapezoidal por requerir corrientes instantáneas discontinuas, pero se preservan durante la comparación como referencia.

6 La aplicación práctica más famosa de esta propiedad es en la mecánica cuántica. El momento y la posición son un par transformado, por lo que la expresión anterior representa el principio de incertidumbre de Heisenberg. 

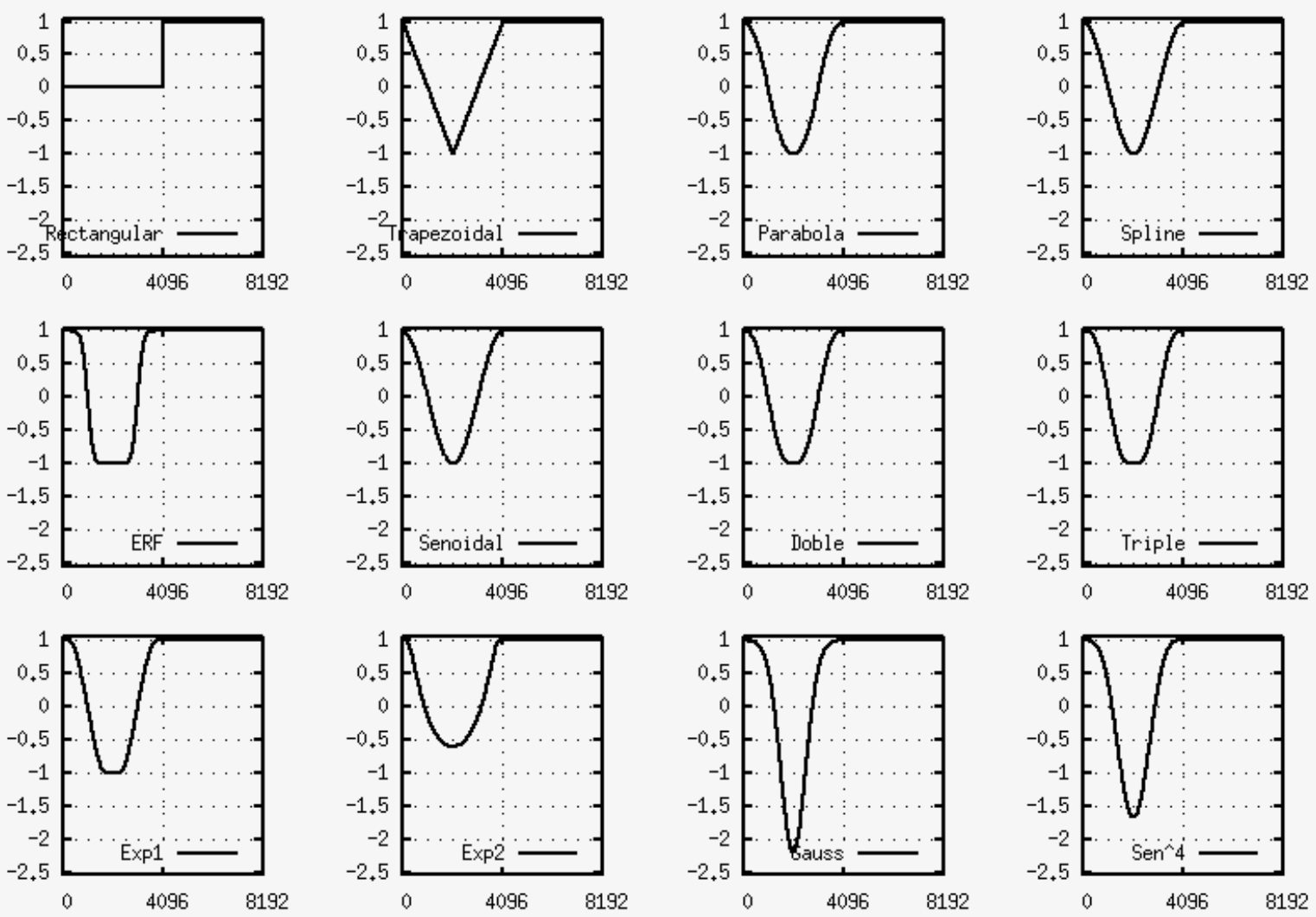

Figura 57: Referencias de velocidad comparadas, incluyendo la zona plana y el retorno, para el caso de un duty-cycle del 50\%. En todos los casos el valor medio es el mismo, lo que permite observar las diferencias en el rango dinámico.

Un método que produce muy buenos resultados es utilizar senos o cosenos de frecuencia ascendente en conjunto con polinomios de distinto orden, como por ejemplo: coseno de período igual al tiempo de retorno, más un offset [senoidal]; seno de la mitad de dicho período superpuesto con una recta de determinada pendiente, más un offset (en dos tramos) [Doble]; coseno de la cuarta parte de período superpuesto con una parábola, más un offset (en cuatro tramos) [Triple].

\section{b) Funciones de empalme}

Las funciones del tipo $f(t)=\exp (-1 / t) o f(t)=\exp (-\exp (-t))$ tienen la propiedad de tener infinitas derivadas nulas en el origen. Pueden utilizarse por tramos teniendo especial cuidado de realizar un empalme cuidadoso en el otro extremo [Exp1].

\section{c) Erfy otras asintóticas}

Otra forma de componer por tramos el retorno es utilizando funciones como $\operatorname{erf}(x)$ o $\tanh (x)$. Estas tienen un muy buen comportamiento en el origen y son asintóticas para valores grandes de $x$, por lo que debe emplearse el tiempo necesario para producir un empalme suave [ERF]. En la Figura 58 puede verse una comparación de la evolución temporal de algunas de ellas. 


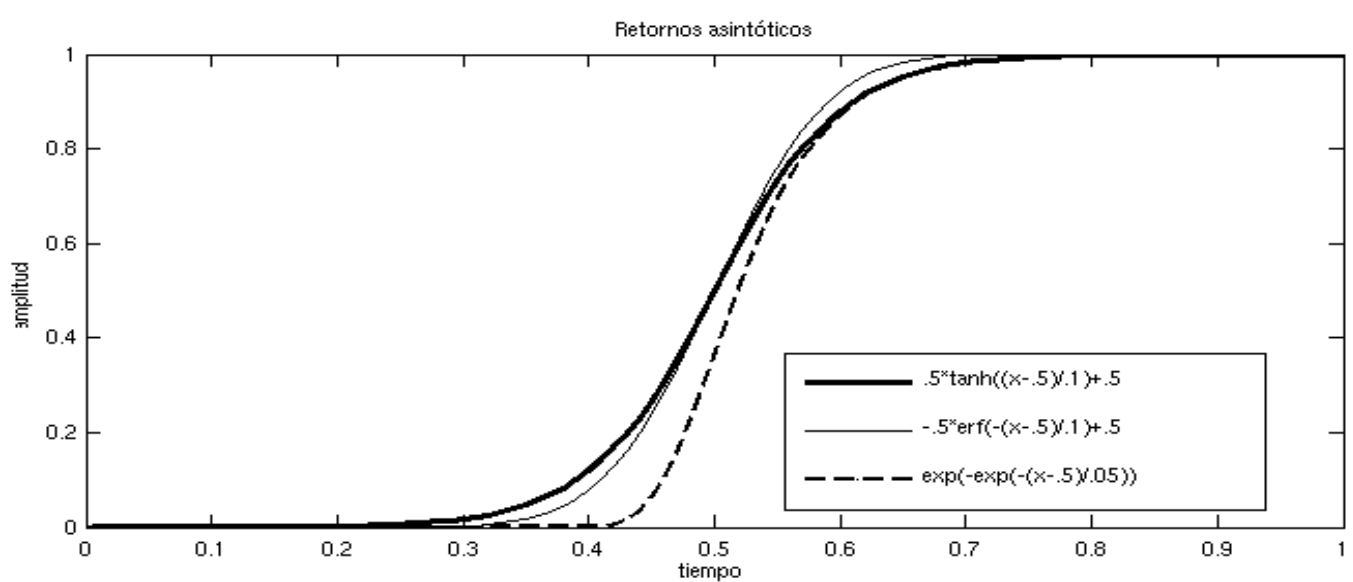

Figura 58: Comparación de la evolución temporal de algunas de las funciones asintóticas utilizadas.

En una segunda categoría pueden colocarse los retornos compuestos por un solo tramo como la gaussiana o los que se obtienen por medio de sucesivas convoluciones o productos de un núcleo o kernel.

\section{d) Gaussiana}

La gaussiana no parece en principio una solución aplicable en este caso pues, si bien cumple con la condición de estar concentrada al máximo en tiempo-frecuencia, es asintótica respecto del eje $x$, lo cual no permitiría un empalme adecuado con la zona plana. Sin embargo, permitiendo un tiempo de establecimiento adecuado presenta buenas características. Ajustando la varianza del retorno se puede modificar el ancho de banda de la señal. Sin embargo debe procederse con precaución en este sentido, ya que debe ajustarse la amplitud para mantener el valor medio y las derivadas crecen rápidamente, al igual que el rango dinámico de la señal [Gauss].

En la Figura 59 puede verse el efecto que tiene la varianza sobre el rango dinámico, la derivada primera y el espectro de frecuencias. Al disminuir la varianza (pulso más angosto) el contenido de alta frecuencia disminuye y el de baja aumenta. El espectro se comprime a expensas de un mayor rango dinámico y de mayores derivadas.

\section{e) Splines}

Una forma elegante de obtener polinomiales por tramos de orden creciente es la siguiente. Partiendo inicialmente de un pulso cuadrado unitario $x(t)$ del ancho del tiempo de retorno se convoluciona con sí mismo, resultando $\operatorname{conv}(x, x)$ triangular del doble de ancho. El resultado puede volver a convolucionarse con $x$, obteniéndose $\operatorname{conv}(\operatorname{conv}(x, x), x)$ polinomial de segundo orden de tres veces el ancho. El procedimiento puede repetirse para obtener splines de mayor orden $^{7}$.

Es importante notar que con cada convolución se aumenta el ancho, por lo que debe comprimirse en la abscisa, implicando que debe aumentarse la amplitud para mantener el mismo valor medio. Aumenta así el rango dinámico de la referencia, dando origen a una mayor velocidad máxima. En la Figura 60 puede verse el efecto en la forma, derivada primera y espectro de frecuencias, hasta el cuarto orden.

7 Puede demostrarse que para un gran número de convoluciones el resultado tiende a la función gaussiana. 


\section{f) Potencias pares del seno}

Otra secuencia interesante es la generada por las potencias pares de un semiciclo de $\operatorname{sen}(x)$. Esta opción tampoco preserva el valor medio, por lo que la amplitud debe ajustarse, produciendo cada vez mayor velocidad máxima al aumentar el orden.

En la Figura 61 puede verse cómo, al igual que en los casos anteriores, se reduce el contenido de alta frecuencia y aumenta el de baja. El espectro se comprime a expensas de un mayor rango dinámico y de mayores derivadas, por lo que debe encontrase un equilibrio. La cuarta potencia presenta un buen balance entre ambas condiciones. Esta se encuentra etiquetada como [Sen^4] en las comparaciones.

\section{g) Otro caso interesante}

El retorno en un tramo compuesto por $\exp \left(-1 /\left(1-x^{2}\right)\right)$ es un caso a tener en cuenta. Muestra que disponer de infinitas derivadas continuas no garantiza el mínimo ancho de banda. Las derivadas son relativamente grandes y presenta un buen valor medio (mayor que la mitad del valor máximo) [Exp2].

\section{Análisis comparativo}

Desde la Figura 62 a la 65 se presentan los resultados de las comparaciones realizadas sobre las referencias más representativas de cada categoría, construidas con igual valor medio y ciclo de trabajo. En las figuras pueden verse las tres primeras derivadas y el contenido armónico representado por la transformada rápida de Fourier.

Respecto de la primer categoría (retorno por tramos con media igual a la mitad del máximo) puede verse que [Triple] presenta tanto un espectro concentrado como derivadas reducidas. En general, puede verse que las soluciones asintóticas producen derivadas relativamente grandes. Al incorporar en la comparación las referencias del segundo grupo (retornos en un tramo con media menor) no se puede ser tan concluyente, ya que presentan algunas ventajas, pero requieren mayor rango dinámico. Por ejemplo [Gauss] tiene el espectro más concentrado, pero las derivadas son mayores; incluso puede verse el efecto del empalme con la zona asintótica. Por otro lado, [Sen^4] presenta un buen balance: la magnitud de las derivadas es comparable con [Triple], el rango dinámico es sólo un 25\% mayor y el espectro está bastante más concentrado. Al incorporar [Exp2] la comparación se complica aun más, pues el espectro está menos concentrado, pero el rango dinámico es menor que en todas los demás casos. En la Figura 66 puede verse un detalle de los espectros de frecuencia de la segunda categoría, dónde puede profundizarse la comparación.

Resumiendo, el retorno [Sen^4] presenta buenas características generales, al tener un buen balance entre rango dinámico, smoothness y concentración del espectro. 

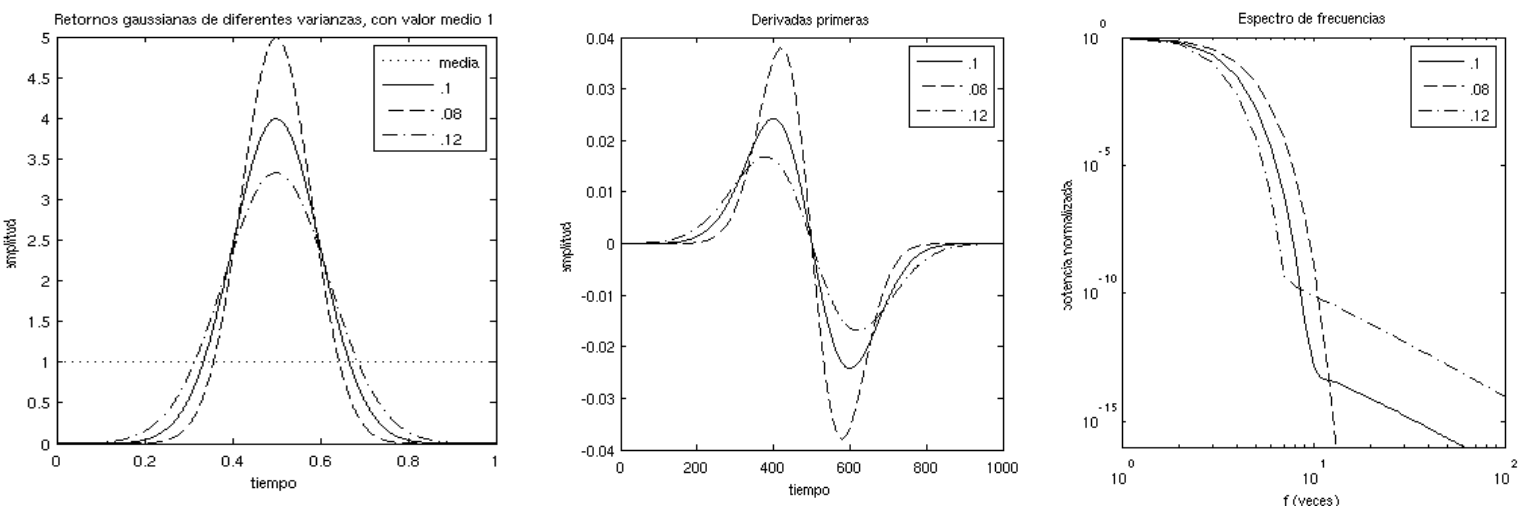

Figura 59: Retornos de la referencia de velocidad con forma gaussiana. Rango dinámico, derivada primera y espectro de frecuencias para tres valores diferentes de varianza. Las amplitudes se adecuaron para obtener valor medio unitario en todos los casos.
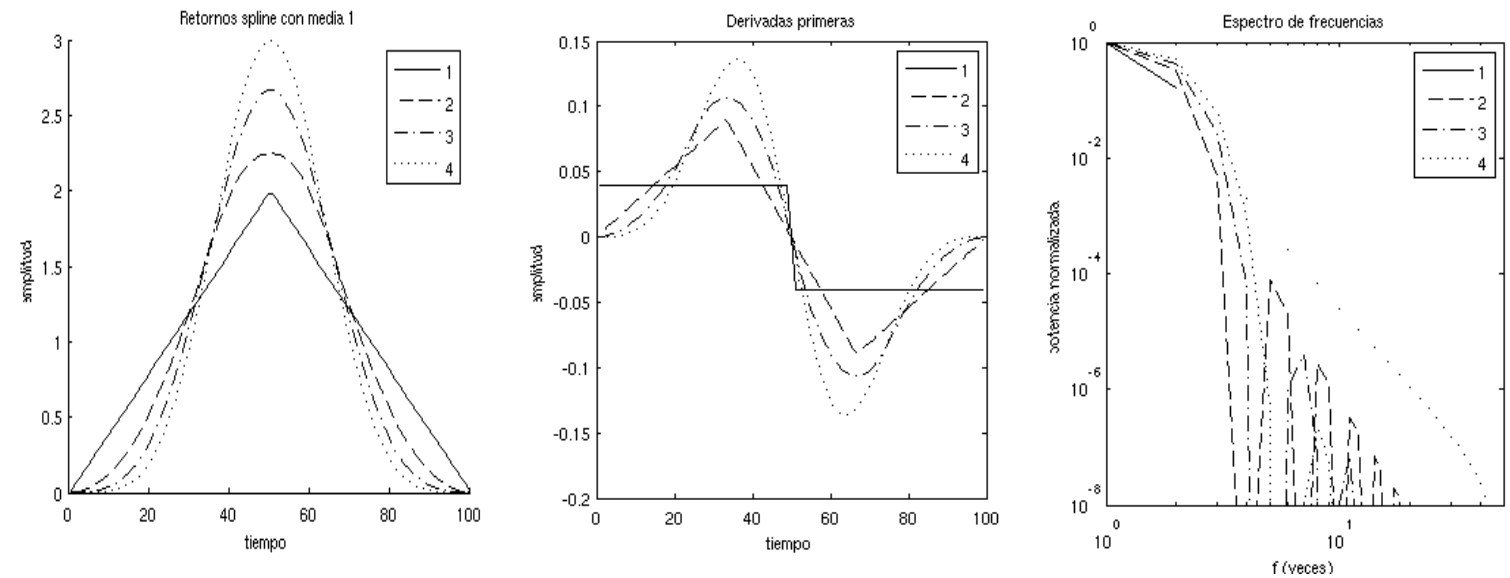

Figura 60: Retornos de la referencia de velocidad con forma spline. Rango dinámico, derivada primera y espectro de frecuencias desde el primero hasta el cuarto orden.Las amplitudes se adecuaron para obtener valor medio unitario en todos los casos.
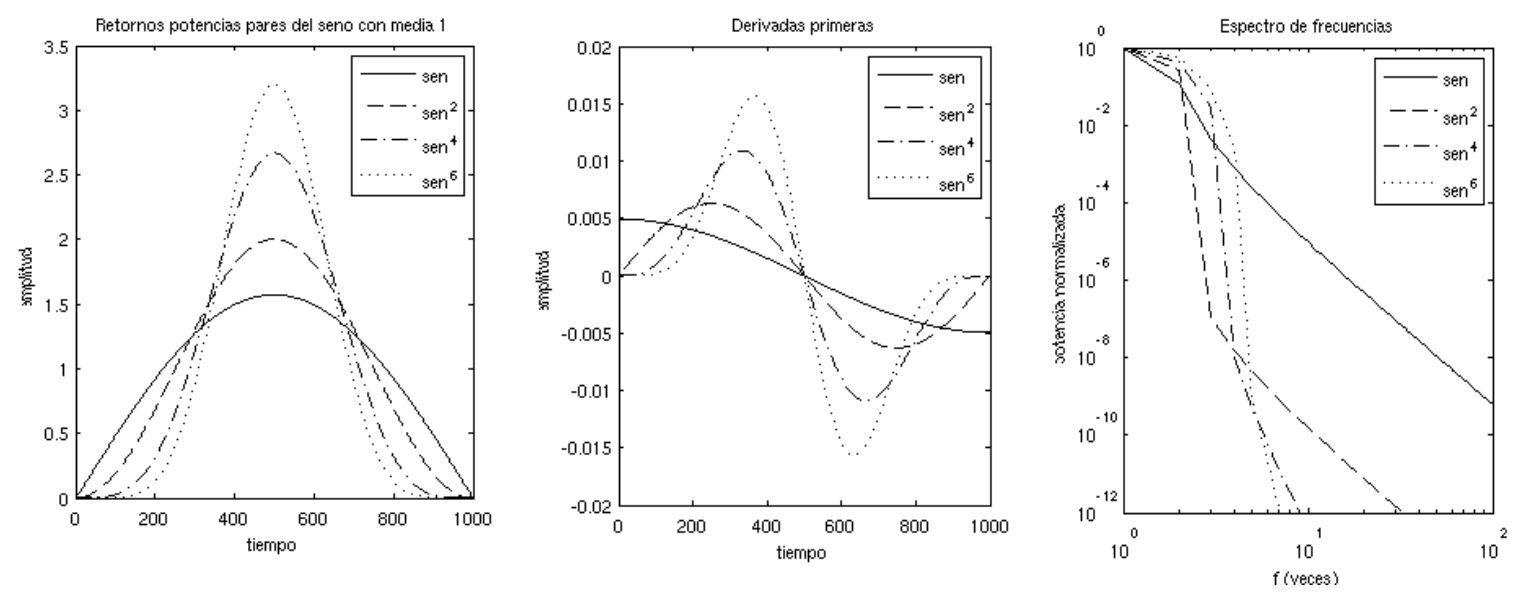

Figura 61: Retornos de la referencia de velocidad con forma de potencias pares del seno. Rango dinámico, derivada primera y espectro de frecuencias desde el primero hasta el sexto orden. Las amplitudes se adecuaron para obtener valor medio unitario en todos los casos. 

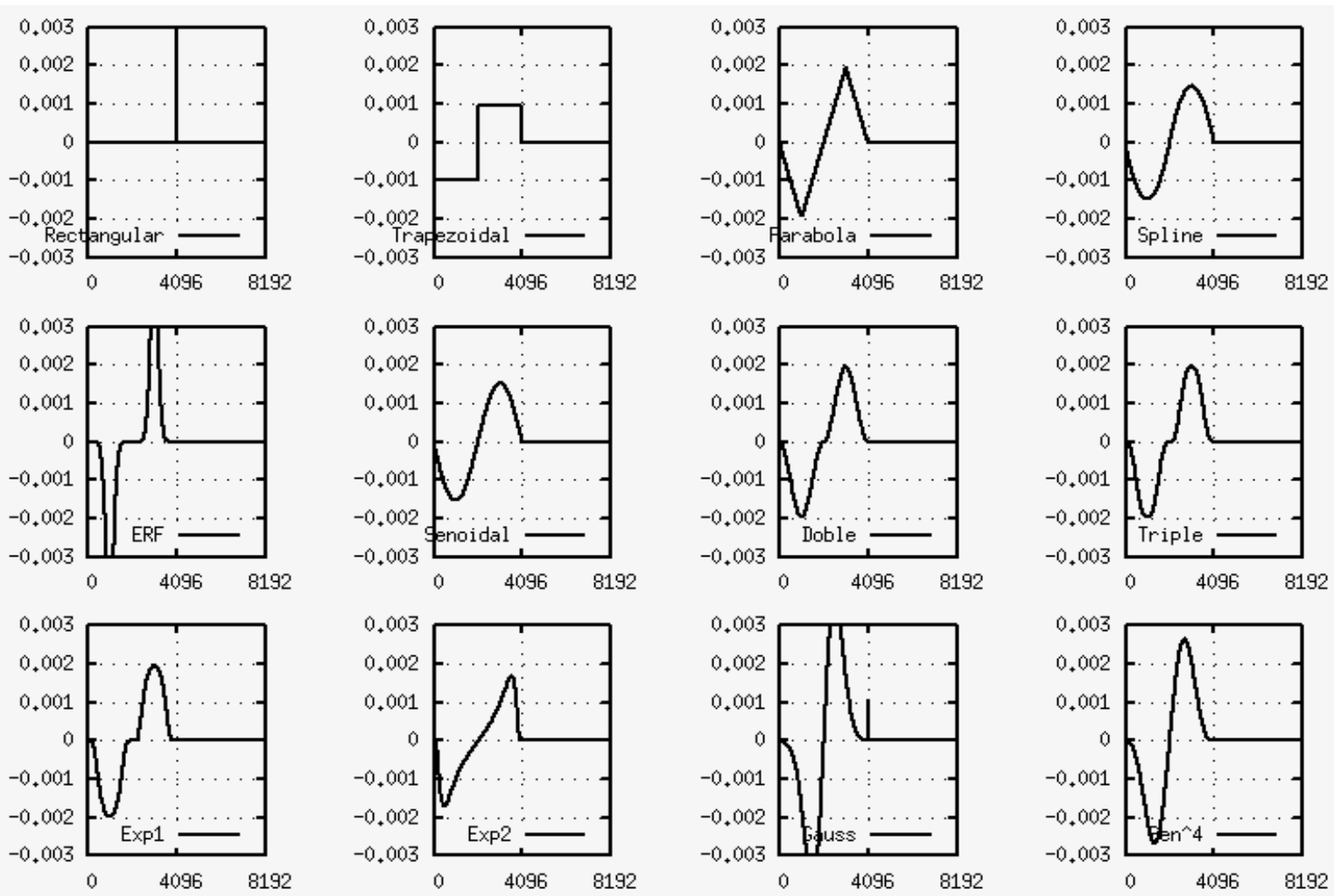

Figura 62: Derivadas primeras de las referencias de velocidad comparadas.
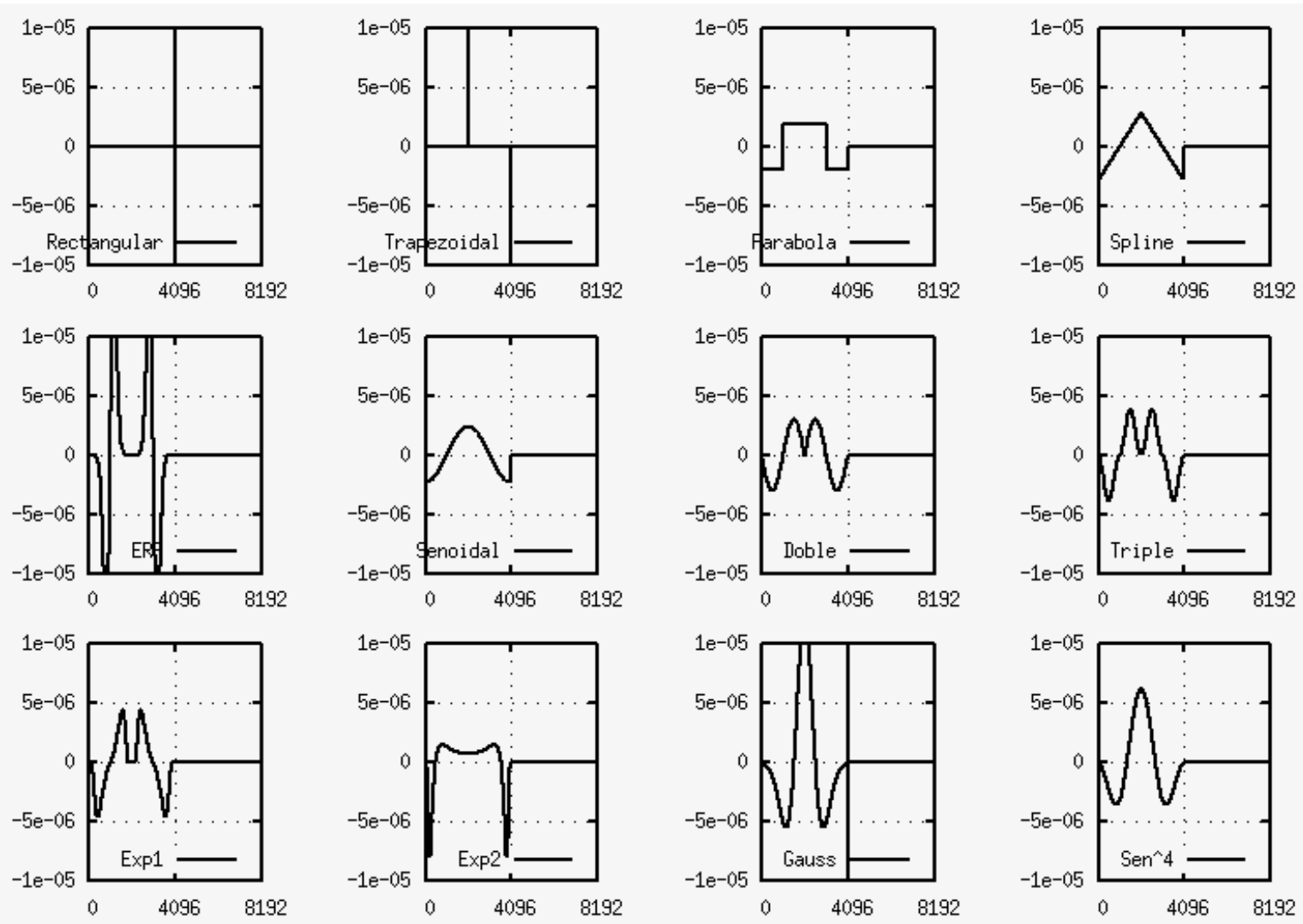

Figura 63: Derivadas segundas de las referencias de velocidad comparadas. 

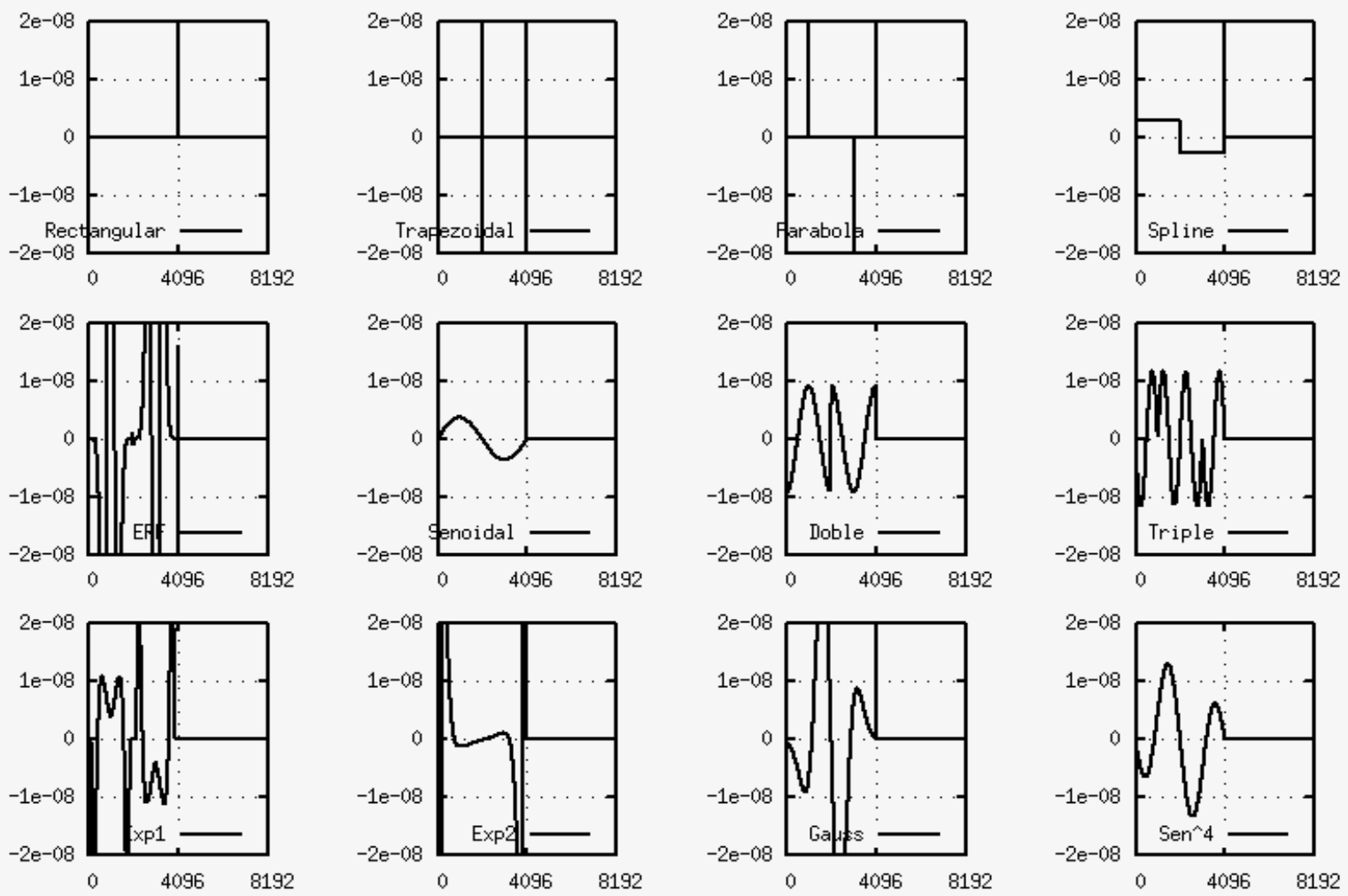

Figura 64: Derivadas terceras de las referencias de velocidad comparadas.
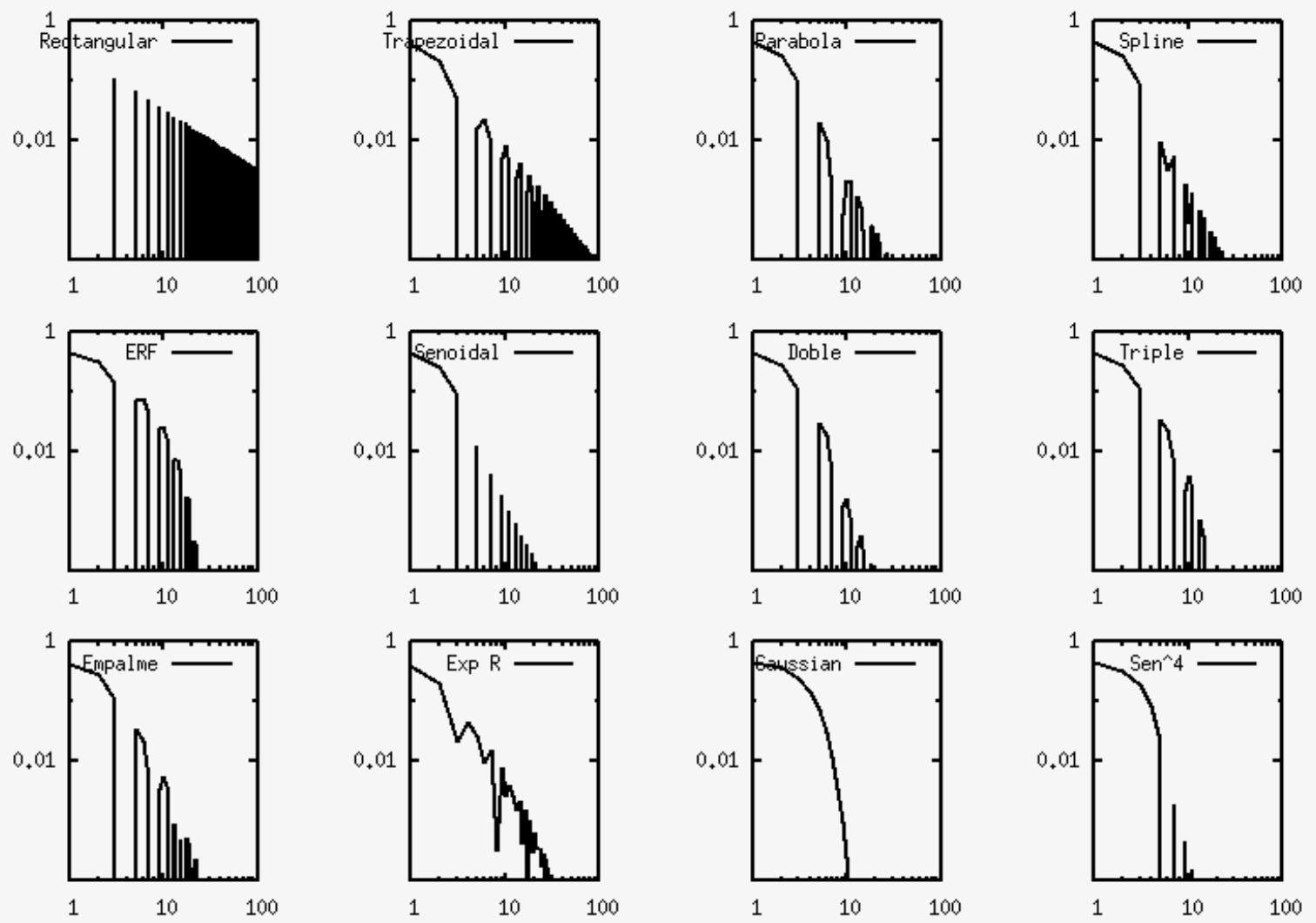

Figura 65: Transformadas rápidas de Fourier de las referencias de velocidad comparadas. 


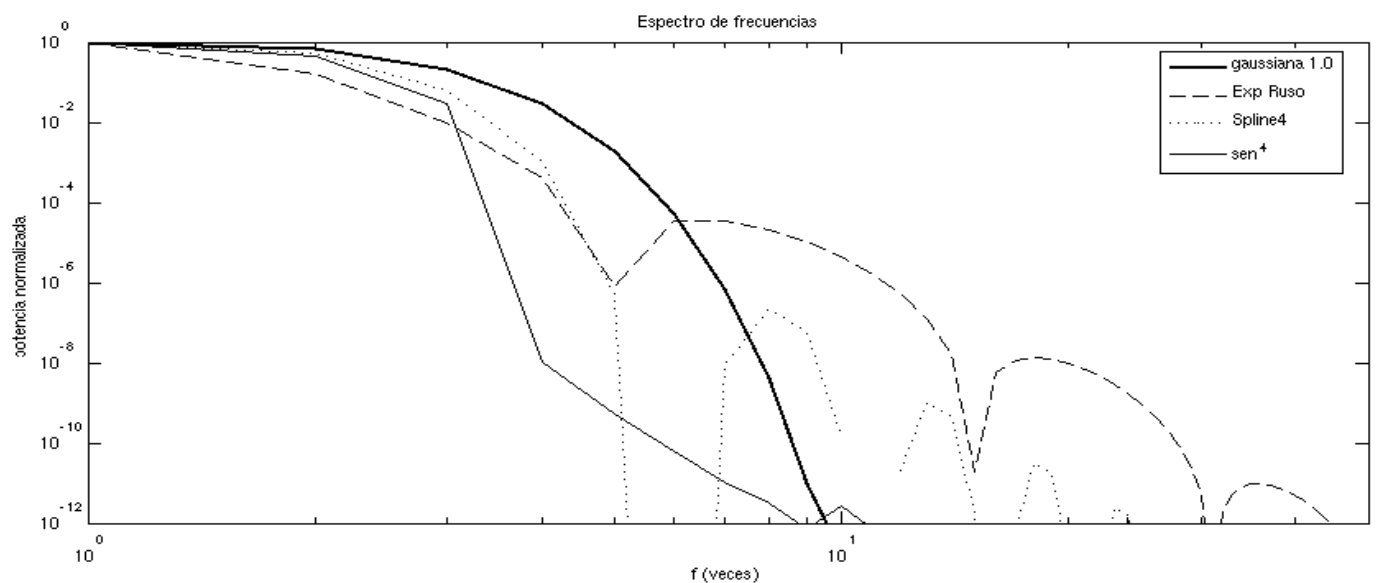

Figura 66: Comparación entre los espectros de frecuencia de la segunda categoría de retornos (ver comp.m)

\subsubsection{Efecto de la cuantización}

Hasta este momento se han utilizado para los estudios funciones continuas, o para ser mas precisos, con doble precisión en punto flotante. En la práctica, éstas serán generadas digitalmente y aplicadas al sistema de control a través de un conversor analógico-digital con la precisión adecuada.

En la Figura 67 se presenta el efecto que tiene la cuantización a 12 y 16 bits en el espectro de frecuencias para un retorno suave.
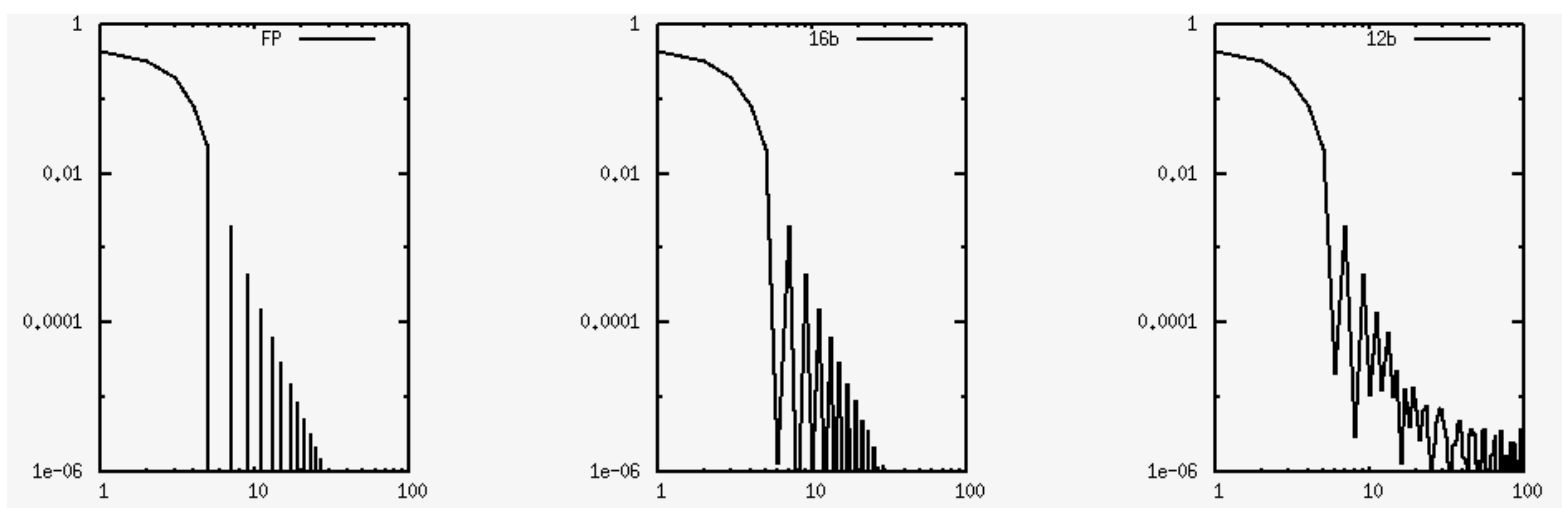

Figura 67: Efecto de la cuantización a 12 y 16 bits sobre el espectro de frecuencias de una referencia con retorno compuesto por potencias pares del seno.

Puede verse cómo los armónicos pares comienzan a hacerse notables a 16 bits, siendo comparables a los impares a 12 bits, pero respetando la envolvente hasta un límite razonable. El efecto de cuantización, más allá de cierto límite, enmascara las diferencias sutiles entre algunas de las formas de onda presentadas, eliminando las pequeñas mejoras que se producen al aumentar el orden, como en el caso de las polinomiales. Las formas con menor valor medio, al requerir un mayor rango dinámico, se verán más afectadas en este sentido.

Respecto del rango dinámico de la referencia es importante notar que la resolución de 12 bits debe preservarse para todas las velocidades. Se desea escalar la referencia a 1000 amplitudes diferentes, por lo menos, preservando sus propiedades. Esto implica al menos otros 10 bits de 
rango dinámico. A fin de evitar la utilización de un conversor digital-analógico de 24 bits, se encontró una solución utilizando un conversor multiplicador de 12 bits con el que se genera la forma de onda base de amplitud constante, accionando sobre su referencia con un segundo conversor de 12 bits. Se obtienen así 4096 velocidades (canales) diferentes. Los detalles de implementación se presentan en el siguiente capítulo.

En la etapa de salida del generador de referencia se incluyó un ajuste manual de la ganancia de tensión a fin de obtener un fondo de escala variable. Esto permite volcar la resolución completa del generador en un rango de velocidades útil. Por ejemplo, si se desea estudiar una zona que va desde los -5 a los $-6 \mathrm{~mm} / \mathrm{s}$, puede seleccionarse $+-6 \mathrm{~mm} / \mathrm{s}$ como fondo de escala. Los 4096 canales se distribuyen linealmente en el rango de $12 \mathrm{~mm} / \mathrm{s}$, obteniéndose más de 330 canales útiles en la zona de interés. Resulta en este caso una resolución en la referencia de velocidad de $0.003 \mathrm{~mm} / \mathrm{s}$, lo cual está un orden por encima de las capacidades del sistema de control de movimiento.

\subsubsection{Selección de la frecuencia y el ciclo de trabajo}

La sintonización de estos dos parámetros para un caso particular es muy dependiente de los detalles del modelo de transductor en cuestión. A continuación se presentarán los lineamientos generales que deberían ser aplicables en todos los casos.

El valor del ciclo de trabajo y la forma de la referencia están relacionados. La forma que requiera una menor acción de control podrá tener un tiempo de retorno menor antes de alcanzar los límites mencionados. Por ello es importante la selección de la forma óptima.

Como se mostró, es deseable concentrar el espectro de frecuencias de la referencia en la zona de mayor ganancia del sistema de control. Para ello es necesario utilizar una frecuencia fundamental de operación levemente menor que la frecuencia de oscilación natural del transductor.

Otra opción podría ser igualar la frecuencia del retorno $1 /(T-F)$ a la frecuencia natural, lo cual funcionaría muy bien con forma sinusoidal. Sin embargo no resulta útil por lo siguiente. Si la frecuencia natural es de aproximadamente $10 \mathrm{~Hz}$ y se desea un ciclo de trabajo de alrededor del $80 \%$, resulta en una zona plana de $0.5 \mathrm{~s}$. Esto implica un desplazamiento excesivo a grandes velocidades (a $10 \mathrm{~mm} / \mathrm{s}$ recorre $5 \mathrm{~mm}$ ), apareciendo los problemas mencionados de ángulo sólido.

Para seleccionar el ciclo de trabajo $F / T$ es importante tener en cuenta que si la relación $T /(T$ $F$ ) es un entero $n$ (el tiempo de retorno es submúltiplo del período) el contenido armónico de la referencia se reduce, pues se reducen notablemente los armónicos pares. Se recomienda entonces utilizar valores para el ciclo de trabajo determinados por la expresión $d=(n-1) / n$ para cualquier $n$ entero, como por ejemplo 50\% (1/2), 66\% (2/3), 75\% (3/4), 80\% (4/5), etc.

En la determinación del valor del ciclo de trabajo existe un compromiso con el transitorio de establecimiento que debe ser anulado con la inhibición de conteo. En el laboratorio se obtuvieron las mayores eficiencias eligiendo un tiempo de retorno tal que se produzca una leve respuesta subamortiguada y utilizando luego el mecanismo de GATE para inhibir el conteo en dicha sección. 
Debe recordarse que el aumento del ciclo de trabajo produce un aumento del valor máximo de la derivada segunda, lo cual implica que se requerirá mayor corriente en el bobinado para seguir la referencia más eficiente, y por lo tanto deberá aplicarse una mayor tensión al bobinado. Evidentemente, dicha corriente no debe superar el límite del arrollamiento de accionamiento, y la tensión debe estar dentro del rango del amplificador de salida de la etapa de potencia. En general, el factor limitante en este aspecto es la acción de control en tensión.

\subsubsection{Resultados}

En el caso concreto de aplicación del transductor Wissel descripto en la Sección 2.2, utilizando un amplificador con +-18 V máximos de salida, se fijó la frecuencia de trabajo levemente menor a la frecuencia natural del transductor $(10 \mathrm{~Hz})$, se utilizó un retorno de potencia cuarta del seno con un ciclo de trabajo del 80\%, con 12 bits de resolución. Resultó en un reducido transitorio sin saturación, que se eliminó con el GATE, resultando en una eficiencia del $75 \%$ con un buen margen en todo el rango de velocidades $(+-10 \mathrm{~mm} / \mathrm{s})$. Los detalles de calibración y mayor precisión sobre los resultados obtenidos se presentarán más adelante.

\subsection{Mecanismo para el cambio de velocidad}

Cuando se opera el transductor a frecuencia constante sin realimentación de posición es importante tener en cuenta que los cambios de velocidad no pueden realizarse en cualquier punto de la trayectoria, pues el nuevo recorrido no se encontraría centrado, a menos que dicho cambio se realice exactamente en el centro. Por ejemplo, si el sistema se encuentra operando a una velocidad alta (gran amplitud de la referencia y gran desplazamiento en ambos sentidos a partir del punto de reposo) y se disminuye abruptamente la velocidad de operación (menor desplazamiento) cuando el eje se encuentra en un extremo del recorrido, el nuevo desplazamiento cíclico se establecerá alrededor del extremo y no del punto de reposo. El control no detecta este corrimiento (sólo sensa velocidad) por lo tanto no puede compensarla. El centro del recorrido evolucionará hacia la posición de reposo con la dinámica natural del sistema, lo cual puede tomar varios segundos.

Este problema, si bien parece evidente, no ha sido abordado previamente, lo cual explica las limitadas prestaciones de los pocos dispositivos programables que aparecen en las publicaciones especializadas. La única forma eficiente de obtener un sistema programable que permita cambios automáticos de la velocidad de medida es sincronizando el cambio de la velocidad de operación con el punto de reposo. El cambio puede realizarse tanto en la zona de velocidad constante como en el retorno, siendo preferible la segunda opción a fin de no perturbar la zona de medida, aunque debe tenerse en cuenta que durante el retorno el eje atraviesa el punto de reposo a mayor velocidad, por lo cual la conmutación debe realizarse con mayor precisión. La Figura 68 muestra la forma que debe presentar la evolución temporal de la referencia durante un cambio de velocidad y los límites espaciales del desplazamiento del eje.

Si el generador de referencia es autónomo y la amplitud del mismo va a ser comandada externamente a través de un mecanismo multiplicador, la solución es proveer una señal de 
sincronización adicional que indique el instante preciso en que el eje atraviesa el punto en que puede recibir un cambio de velocidad. En la figura se muestra dicha señal (TRIGGER_OUT) junto con la señal de habilitación para el contador (GATE_OUT).
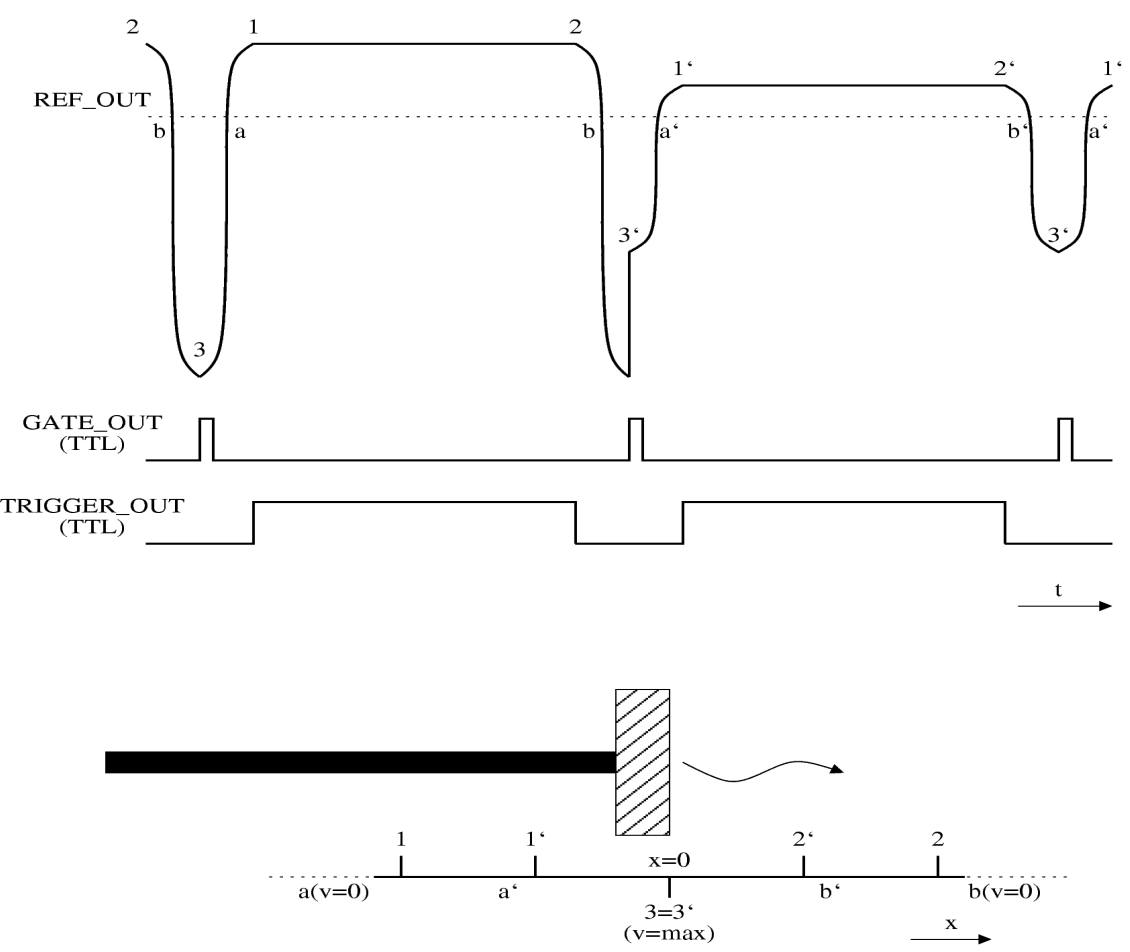

Figura 68: Mecanismo de cambio de velocidad para el accionamiento a frecuencia constante sin realimentación.

Si se supone que el sistema de control se comporta en forma lineal, el transitorio que se presenta ante un cambio de velocidad tiene una amplitud relativa proporcional a la magnitud del cambio. Para cambios grandes la amplitud absoluta del transitorio es mayor que para cambios pequeños en la velocidad. Esto sugiere que debe ponerse especial cuidado en la estrategia que se utiliza para recorrer los distintos canales de interés, evitando trabajar en forma alternada. Incluso debe ponerse especial cuidado cuando se desea invertir el signo de la velocidad, ya que este fenómeno produce los transitorios más prolongados. En este último caso puede ser recomendable detenerse en velocidad nula durante un período antes de solicitar una velocidad con signo contrario.

\subsection{Especificación del generador de referencia}

A modo de resumen de lo expuesto en este capítulo se presentan las especificaciones para el generador de referencia, cuyos detalles de diseño se presentarán en el contexto del próximo capítulo.

i. Resolución de la referencia de al menos 12 bits para todo el rango de velocidades.

ii. Al menos 1000 canales (velocidades diferentes).

iii. Ajuste manual de precisión del valor medio nulo. 
iv. Selección de la frecuencia de la referencia de velocidad, para que pueda ser sintonizada con la frecuencia natural de oscilación del transductor. De ser posible, que la frecuencia de operación pueda elevarse por encima de los $100 \mathrm{~Hz}$ con el único objetivo de facilitar la calibración del valor medio nulo con un voltímetro.

v. Múltiples formas de retorno para que el usuario pueda seleccionar la más adecuada para cada caso.

vi. Selección del ciclo de trabajo (tiempo de retorno ajustable).

vii. Inhibición del conteo y el tiempo neto de medida durante el retorno, más un tiempo adicional programable (GATE). La señal de inhibición debe ser observable, para que el sistema pueda ser calibrado con un osciloscopio.

viii.Realimentación discreta de posición, para sincronizar el centro o los extremos y así poder acoplar el control en continua (combinación de ambas técnicas opcional).

ix. Mecanismo de sincronización de los cambios de velocidad con el centro del recorrido, para evitar respuestas transitorias indeseables.

x. Etapa de salida de ganancia variable, para poder seleccionar el fondo de escala

La selección de los valores adecuados para los puntos v, vi y vii representan un compromiso. Debe encontrarse el óptimo para cada caso de aplicación particular. Con un osciloscopio, debe ser posible observar simultáneamente el error del sistema de control y la inhibición del conteo y el tiempo. La combinación trayectoria/ciclo de trabajo que requiera menor tiempo de inhibición es la mejor, por ser la de mayor eficiencia.

Utilizando instrumental de laboratorio de precisión (generadores de señal con lógica adicional) debería ser posible implementar una combinación de dispositivos que cumplan con estas especificaciones. Sin embargo es deseable que el generador de referencia se encuentre integrado y en perfecto sincronismo con la unidad de procesamiento, por lo cual ambos bloques se diseñaron en conjunto. Los detalles de construcción se presentarán en el próximo capítulo.

Para finalizar se enumerarán los aportes originales que se han presentado en este capítulo, en vistas a la operación de transductores electromecánicos Mössbauer a velocidad constante:

i. Modelado del sistema a controlar y diseño de un compensador para la operación a lazo cerrado con realimentación de velocidad.

ii. Incorporación de un mecanismo de realimentación de posición discreta de simple implementación.

iii. Especificación de la óptima referencia de velocidad.

iv. Mecanismo de sincronización para el cambio de velocidad.

Estos cuatro aspectos de diseño pueden ser combinados para obtener un sistema de máximas prestaciones. Se ha abierto así la posibilidad de operación programable de los transductores comerciales a velocidad constante, lo cual no se había realizado eficientemente hasta el momento.

De esta manera se completa el diseño y especificación de la etapa de accionamiento del 
espectrómetro Mössbauer de velocidad constante programable, objetivo de esta tesis. La implementación de la instrumentación asociada se detalla en el siguiente capítulo, en el contexto de la etapa de procesamiento. 


\section{Capítulo 5 \\ La etapa de procesamiento programable}

Los componentes presentados en este capítulo representan el núcleo de operación automática por medio del cual se completa el espectrómetro Mössbauer de velocidad programable propuesto inicialmente. Su principal objetivo es implementar el nuevo modo de funcionamiento, en interacción con el resto de las etapas optimizadas previamente. Es importante notar que no se trata de la adecuación de instrumental nuclear estándar, sino de un instrumento completamente nuevo, diseñado en función de los requerimientos que surgen de la operación a velocidad programable. El diseño es una combinación de dispositivos digitales y analógicos de precisión, para el cual se utilizaron técnicas de embedding y tiempo real a fin de obtener un sistema versátil y completamente programable, que satisface los requerimientos planteados. La base de operación en tiempo real es la presentada previamente en la Tesis de Magister [Veiga 1999].

\subsection{Consideraciones generales de diseño}

Según la propuesta presentada en la Sección 2.5, la etapa de procesamiento debe incluir al menos la siguiente funcionalidad:

i. Conteo de eventos en función del tiempo (ratímetro). Los eventos a contar están representados por pulsos lógicos provenientes de la etapa de detección, la cual está compuesta por un contador proporcional, un preamplificador, un amplificador de conformación y un discriminador diferencial, tal como se describió en la Sección 2.1 y se optimizó en el Capítulo 3. El discriminador provee los pulsos lógicos a partir de los cuales debe calcularse la tasa.

ii. Generación de la referencia analógica de velocidad constante. Ésta representa la entrada de la etapa de accionamiento, compuesta por el transductor electromecánico y 
su control de velocidad, tal como se describió en la Sección 2.2. La referencia debe ser reconfigurable y el valor de la velocidad constante de operación debe poder ser seleccionado en forma automática. Las especificaciones para la referencia se elaboraron a lo largo del Capítulo 4.

iii. Sincronización interna entre el conteo y la referencia de velocidad constante. La tasa de eventos registrada debe asociarse con exactitud a una única velocidad de medida. Todos los aspectos de operación que impliquen restricciones temporales estrictas deben ser resueltos por esta etapa de sincronización, a fin de liberar al resto de los componentes de la operación en tiempo real.

iv. Comunicación hacia el exterior en modo interactivo o en modo automático a través de una interfaz estándar, de modo que el espectrómetro pueda ser operado tanto en forma manual como utilizando un programa de automatización alojado en una computadora personal. Los tiempos de respuesta involucrados en esta comunicación no deben ser determinantes de la calidad de la medida.

v. Almacenamiento no volátil de los parámetros de configuración, monitoreo de estado y otros aspectos funcionales de menor relevancia, incluyendo la integración en un único módulo NIM, al cual se lo denominó escalímetro de velocidad programable.

En la Figura 69 se presenta un diagrama esquemático que resume la funcionalidad descripta y presenta los dos niveles diferenciados de comunicaciones. El diseño fue realizado de forma de resolver internamente todas las tareas con especificaciones estrictas de tiempo, como son la sincronización de la referencia con los eventos contados, la inhibición del conteo durante el retorno del eje, la sincronización para el cambio de velocidad o la realimentación de posición opcional para el modo de desplazamiento constante.

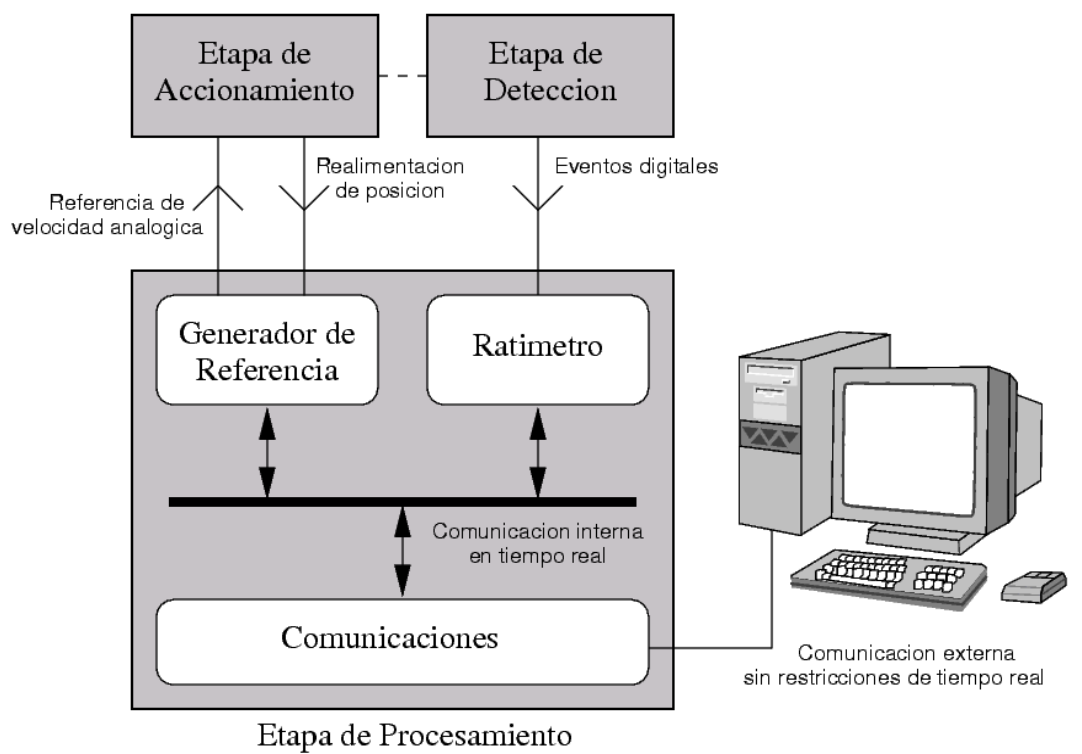

Figura 69: Diagrama funcional y flujo de la información para el escalímetro de velocidad constante que compone la etapa de procesamiento. 


\subsubsection{Arquitectura propuesta}

Para la implementación de la funcionalidad descripta se diseñó una arquitectura con dos microcontroladores comunicados a través de un bus interno. En la Figura 70 se presenta un diagrama esquemático de la configuración propuesta.

Un procesador principal se encarga de las comunicaciones externas, sincronización e implementación del ratímetro. Se utiliza un procesador auxiliar para almacenar las formas de onda de retorno y generar la forma base de la referencia, utilizando un conversor digitalanalógico de 12 bits multiplicador. La entrada de referencia de este último se maneja utilizando los conversores digital-analógico integrados en el procesador principal, en sincronismo con la detección de eventos.

Como se mostró en el capítulo anterior, la configuración con doble conversor DA tiene su base en que son necesarios al menos 1000 canales de velocidad diferentes, y en cada uno de ellos la resolución de la referencia debe ser de 12 bits. Esto implica al menos 22 bits de rango dinámico. Para evitar la utilización de un conversor de 24 bits, se utiliza un dispositivo multiplicador de 12 bits de buena calidad, con su referencia manejada por otro de 12 bits (este puede permitirse de menor calidad pues sólo son necesarios 10 bits). La base del diseño del generador fue presentada en [Veiga 2002]. En este capítulo se describe brevemente y se muestra su integración con el conjunto.

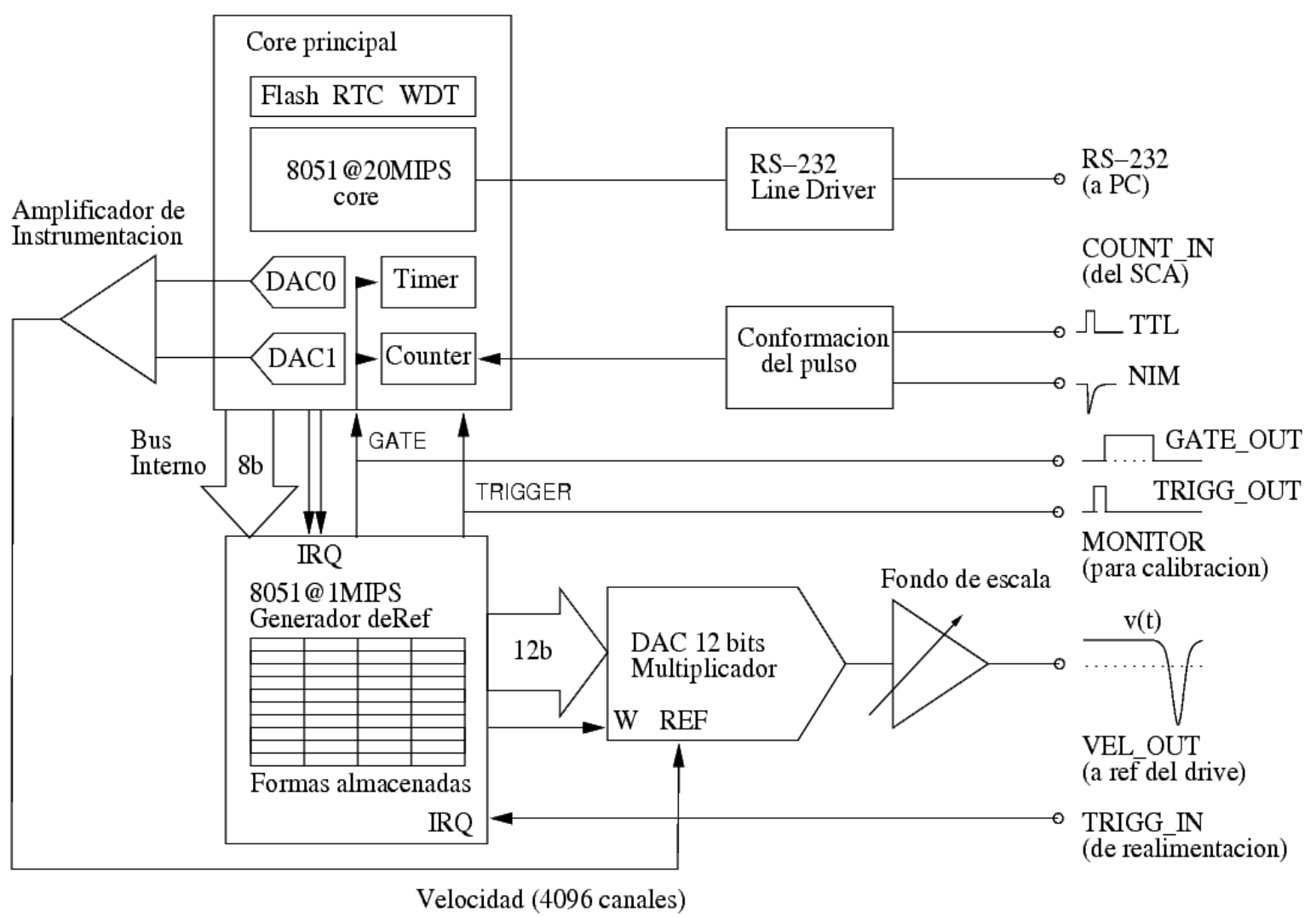

Figura 70: Diagrama en bloques de la arquitectura dual propuesta para la etapa de procesamiento. 
La comunicación externa se implementa a través de un enlace serie que será manejado manualmente en modo interactivo o por medio de un lenguaje de programación de alto nivel sin especificaciones estrictas.

Para la comunicación entre los procesadores se implementó un bus paralelo con 8 bits de datos (que ocupan un puerto de cada microcontrolador) y dos bits de control (uno de ellos asociado a una interrupción a fin de generar el sincronismo). Este bus es utilizado por el procesador principal para modificar los parámetros del generador de referencia.

Todos los parámetros del experimento pueden ser configurados remotamente y almacenados en memoria no volátil en el procesador principal. El procesador auxiliar no dispone de almacenamiento permanente, siendo configurado en el arranque con los parámetros almacenados.

Entre las salidas se provee el mecanismo necesario para el ajuste del GATE con osciloscopio, lo que debe hacerse en conjunto con la observación de la señal de error provista por el control de velocidad. También se provee de un conector externo para la señal digital proveniente del sensor discreto de posición.

Para explotar al máximo la resolución del instrumento se provee de un control manual de la velocidad correspondiente al último canal, a través del amplificador de salida. Se incluye además la conformación de los pulsos provenientes del discriminador. Los detalles de diseño más relevantes de cada bloque se muestran en lo que resta del capítulo.

\subsubsection{El procesador principal}

Como procesador principal se utilizó un microcontrolador de 8 bits rápido (single-cycle a 20 $\mathrm{MHz}$ ) que incluye dos conversores digitales-analógicos de 12 bits integrados. Su arquitectura es compatible con la línea de microcontroladores 8051/8052 de Intel. Los criterios de selección se exponen a continuación.

i. La arquitectura es bien conocida y se dispone de adecuadas herramientas de programación.

ii. Con cualquiera de los modelos compatibles disponibles en el mercado se puede realizar una buena interfaz de usuario a través de un enlace serie de comunicaciones (RS232, BlueTooth, etc.).

iii. Dispone de contadores/timers de 16 bits con los que se puede implementar el ratímetro. Como se utiliza el ciclo de reloj como referencia, es necesario utilizar un procesador de al menos $10 \mathrm{MHz}$ para obtener un tiempo muerto y un error en la base de tiempo de alrededor de $100 \mathrm{~ns}$.

iv. Puede disponerse en algunos modelos de uno o varios conversores DA integrados para comandar el generador de referencia. En general éstos no son de muy buena calidad, pero tienen una resolución de 12 bits, mientras que sólo se necesitan 10 para obtener los 1000 canales especificados.

v. En este rumbo se descarta generar la referencia de precisión con el procesador principal. Dados los estrictos requerimientos presentados al final del Capítulo 4 para el generador, se justifica utilizar un microcontrolador dedicado o procesador auxiliar. 
Una vez liberado el procesador principal de la tarea de generar la forma base de la referencia de velocidad, puede seleccionarse el microcontrolador ADuC841 de Analog Devices. Se trata de un núcleo compatible con la arquitectura 8051 de Intel, con las siguientes características:

i. Suficiente capacidad de memoria: $62 \mathrm{~KB}$ de EEPROM para programas, $2.25 \mathrm{~KB}$ de RAM para datos y $4 \mathrm{~KB}$ de memoria FLASH para almacenamiento permanente de parámetros de operación.

ii. Tres timers/counters de 16 bits.

iii. Suficiente velocidad de procesamiento (single-cycle a $20 \mathrm{MHz}$, lo que equivale a 20 MIPS). Esto permite disponer de una adecuada resolución en el contador y en la base de tiempo (ver a continuación).

iv. Sistema de interrupciones extendido, con dos niveles de prioridad.

v. Dos conversores DA de 12 bits de regular calidad, con referencia de tensión interna.

vi. UART para comunicaciones serie.

vii. Otras prestaciones de utilidad incluyen: TIC (Time Interval Counter), WDT (Watchdog Timer), PSM (Power Supply Monitor), PDM (Power Down Mode), PLL on-chip programable para controlar la frecuencia de operación y monitor de temperatura.

viii.Otras prestaciones que no son de utilidad en este caso: conversor AD de 8 canales con DMA, y puede operar con alimentación de $3 \mathrm{~V}$ (lo cual no es conveniente en este caso pues se degrada el rango dinámico de los conversores DA).

La arquitectura 8051 de Intel fue estudiada en profundidad en la Tesis de Magister anterior [Veiga 1999]. Para ésta se implementó un micro-kernel utilizando el sistema de interrupciones disponible en dos niveles, que permite la operación en tiempo real (ver a continuación).

\subsubsection{El procesador auxiliar}

Un segundo procesador con menores prestaciones es utilizado como núcleo del generador de referencia. Este debe almacenar las diferentes formas de retorno y comandar el conversor DA multiplicador de forma de utilizar todo su rango dinámico.

Se utiliza uno de los tantos dispositivos disponibles en el mercado que son $100 \%$ compatibles con el microcontrolador 8051 de Intel, en este caso el AT89C51 de Atmel, operando a 12 MHz. Con 12 ciclos de reloj por ciclo de máquina, permite disponer de 1 MIPS, lo cual es adecuado para la aplicación. Si se supone que se necesitan entre 5 y 10 instrucciones por iteración que maneja el conversor DA, permite obtener una frecuencia de actualización de $100 \mathrm{KHz}$. Suponiendo que se opera el transductor con una frecuencia fundamental de $10 \mathrm{~Hz}$, con una eficiencia del 90\% (peor caso), permite disponer de 1000 actualizaciones del DA por cada retorno.

Para más detalles acerca del funcionamiento de este dispositivo, ver a continuación la implementación del generador de referencia. 


\subsection{El ratímetro}

Este módulo funcional se compone de un contador y una base de tiempo. La gran mayoría de sus requerimientos provienen del Capitulo 3, como la especificación de la señal de entrada, tiempo muerto, resolución mínima en tiempo y máximo numero de eventos que debe poder manejar. Otras especificaciones provienen del Capítulo 4, como la inhibición sincronizada o GATE.

\subsubsection{El contador}

Respecto de la señal de entrada, debe tenerse en cuenta que un discriminador diferencial estándar, como por ejemplo el modelo 550A de Ortec, tiene dos salidas:

- Una salida rápida que utiliza el estándar de pulsos NIM de corriente negativos de 20 ns de ancho y rise-time menor que 5 ns, con una resolución entre pulsos de 200 ns.

- Una salida lenta TTL de 500 ns de ancho y 800 ns de resolución entre pulsos, con un rise time de $20 \mathrm{~ns}$.

El contador integrado del ADuC requiere que el pulso sea TTL y esté por lo menos un ciclo de reloj en nivel bajo y un ciclo en nivel alto, para que pueda ser detectado. Si el reloj es de $20 \mathrm{MHz}$ (el ciclo de reloj es de $50 \mathrm{~ns}$ ) en ninguno de los dos casos habrá problemas de tiempo.

El único inconveniente es de conformación en el caso de la salida NIM. Debe convertirse un pulso NIM de $20 \mathrm{~ns}$ en un pulso TTL de al menos $50 \mathrm{~ns}$ de ancho. Dado que se dispone de tiempo, ya que el tiempo muerto del discriminador es de $200 \mathrm{~ns}$, el pulso NIM se conformó a un TTL de $100 \mathrm{~ns}$ de on. El tiempo de off, necesario para que pueda ser detectado por el microcontrolador los impone el tiempo muerto del discriminador (otros $100 \mathrm{~ns}$ ).

El circuito de conformación se basa en el comparador ultra rápido AD96685 de Analog Devices (2.5 ns de tiempo de propagación) para la detección del nivel, más lógica rápida de $100 \mathrm{MHz}$ (un Flip-Flop D con reset 74F74 y varias compuertas) con que se construyó un circuito monoestable para ensanchar el pulso. El retardo (que debe ser calibrado para ser mayor que $50 \mathrm{~ns}$ ) se compone de seis inversores provistos por el integrado 7406 y dos redes R-C. El esquemático puede verse en la Figura 71, junto con su conexión a la entrada T1 del contador interno del ADuC. No se utiliza un monoestable integrado pues no existen dispositivos que se exciten con un pulso de entrada tan corto.

En la implementación final del contador se utilizó un selector manual para permitir la entrada tanto de pulsos de NIM como TTL. En esta etapa puede incluirse un discriminador integrado de bajo costo, cuyo diseño se presenta en el Apéndice A. En este caso no se consideró necesario, pues la etapa de detección optimizada anteriormente incluyó un discriminador NIM disponible. 


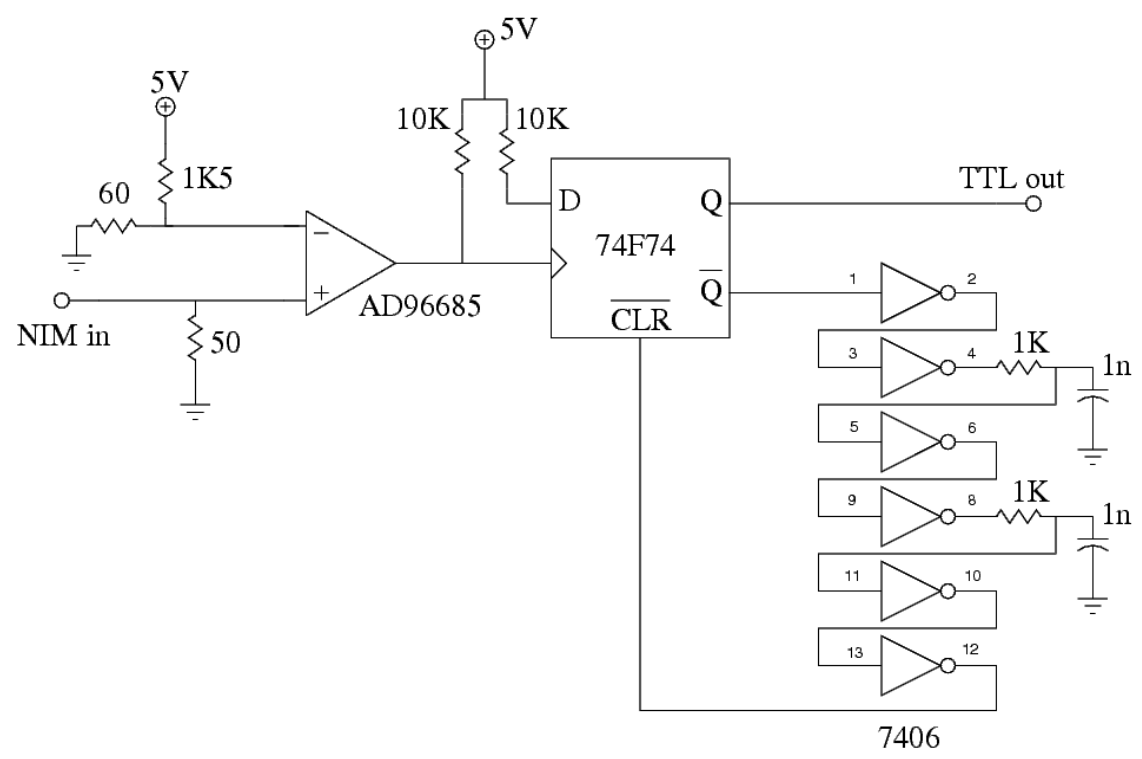

Figura 71: Circuito de conformación de los pulsos NIM de salida de un discriminador Ortec a la entrada TTL del microcontrolador.

Para la implementación del contador se utilizó el timer/counter 1 del ADuC en modo contador de 16 bits, conectando la salida del circuito de conformación a la entrada T1 del AduC841. Para ampliar el rango se manejó por software la excepción generada ante un desborde. De este modo pudo implementarse un contador de eventos extendido de 24 bits utilizando uno de los registros internos de 8 bits. Si la tasa se encuentra en el orden de las decenas de $\mathrm{KHz}$, éste permite el almacenamiento de eventos durante varios minutos operando a una misma velocidad.

\subsubsection{La base de tiempo}

Es responsabilidad de la unidad de procesamiento el mantener una base de tiempo unificada para las medidas que se realizan independientemente por canal. El intervalo de tiempo asociado al canal debe ser muy preciso, a la vez que debe ser programable.

Para su implementación se utilizó el timer/counter 0 del microcontrolador en modo timer de 16 bits (ver Figura 72) manejando su desborde por medio de la interrupción asociada. Se programó una base de tiempo generando una interrupción cada $2.5 \mathrm{~ms}$ (unidad mínima de tiempo de conteo). Con un registro de 16 bits programable a través de la interfaz de usuario (scaler) se selecciona el período neto de conteo, hasta un valor máximo de casi tres minutos ( $2^{16}$ veces $\left.2.5 \mathrm{~ms}\right)$.

Para implementar el mecanismo de inhibición del conteo desde el generador (GATE) se utilizó el mecanismo de deshabilitación por hardware disponible en el ADuC (entradas /INT0 e /INT1 cableadas directamente a la salida GATE_OUT del generador de referencia). De este modo, tanto la base de tiempo como el contador pueden detenerse simultáneamente ante una entrada nula de GATE, con una precisión de 50 ns. Por lo tanto el tiempo que contabiliza el microcontrolador es el tiempo efectivo de medida. 


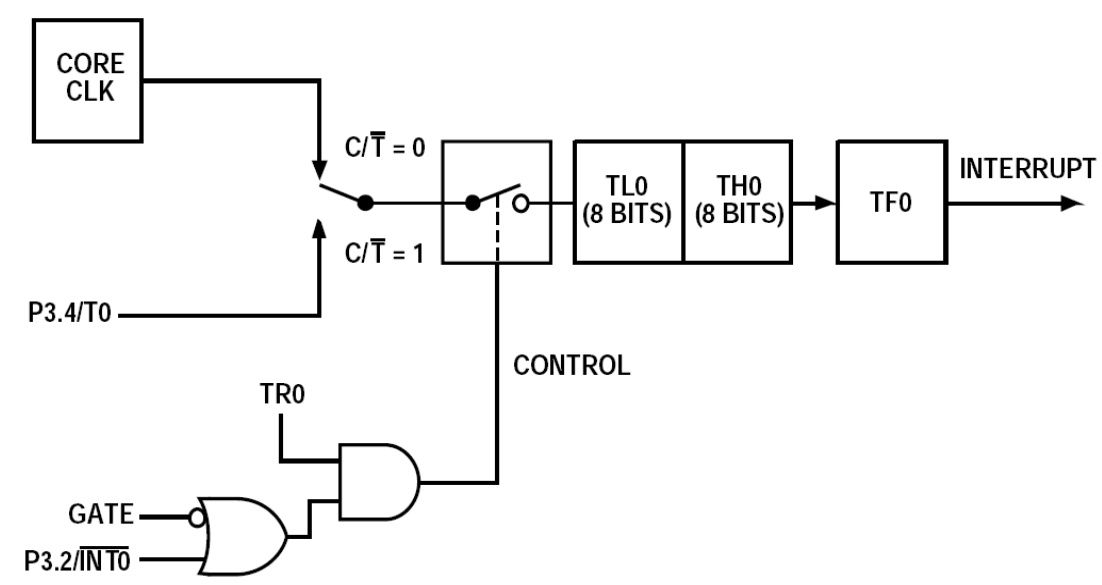

Figura 72: Timer 0 del ADuC841 en Modo 1 (16 bits) con el mecanismo de inhibición vía P3.2. $C / T=0$ para el timer y $C / T=1$ para el counter [Analog Devices AduC841 datasheet].

\subsubsection{Prestaciones}

Respecto del tiempo muerto del contador, debe tenerse en cuenta que el hardware disponible en el microcontrolador es extendible. Si los pulsos tienen un período menor que el ciclo de reloj del procesador, no se cumple necesariamente la condición de conteo (un ciclo en nivel alto y el siguiente en nivel bajo). Pero como los pulsos provienen de un dispositivo cuyo comportamiento respecto del tiempo muerto es no extendible, es suficiente con que el contador tenga un período de reloj de la mitad del tiempo muerto del discriminador. Esta condición se cumple holgadamente con el microcontrolador operando a $20 \mathrm{MHz}$. El monoestable garantiza los $50 \mathrm{~ns}$ en nivel alto (utiliza $100 \mathrm{~ns}$ ), y el tiempo muerto del discriminador no extendible (200 ns) garantiza el nivel bajo (otros $100 \mathrm{~ns}$ ). Resulta una tasa máxima de conteo uniforme de $5 \mathrm{Mc} / \mathrm{s}$. En conclusión, en estas condiciones el contador no agrega tiempo muerto a la etapa de adquisición. El tiempo muerto de la etapa está impuesto principalmente por el amplificador de conformación, como se mostró en el Capítulo 3.

Respecto del conteo, la tasa de pérdidas puede ser de hasta uno por período de operación del transductor: el GATE inhibe antes de que se cumpla la condición de un ciclo en nivel bajo. Esto implica una pérdida de eventos de $10 \mathrm{~Hz}$ aproximadamente. Si la tasa es 10K, representa un error sistemático en el conteo de media $-0.1 \%$, el cual puede despreciarse sin problemas.

No se consideró necesario que las bases de tiempo de ambos procesadores estén sincronizadas. Cada uno opera con su propio oscilador. Es importante tener en cuenta la estabilidad en frecuencia de ambos cristales. Sobre todo el del procesador principal, que se encarga de la base de tiempo. Un corrimiento en la frecuencia modifica la tasa de conteo. Pero si la frecuencia es estable con el tiempo no afecta las medidas, pues se trata de tasas relativas. Sólo debe considerarse el drift del cristal. Un dispositivo de moderada estabilidad $(10 \mathrm{ppm})$ satisface este requerimiento.

\subsection{El generador de referencia}

Los requerimientos provienen del final del Capítulo 4. Las estrictas especificaciones de 
linealidad, resolución, rango dinámico y sincronización justifican la utilización de un core independiente que se encargue que la generación de la referencia. Se utilizó como base el generador autónomo diseñado para la Tesis de Magister [Veiga 1999] [Veiga et al 2002], y fue utilizado en configuraciones experimentales tanto en la UNLP como en los laboratorios del Grupo de Física Experimental y Bajas Energías del CBPF (Centro Brasilero de Investigaciones Físicas). En esta sección se describe brevemente su funcionalidad y prestaciones, para luego mostrar su integración con la etapa de procesamiento.

\subsubsection{Implementación autónoma}

El microcontrolador auxiliar genera las diferentes formas de referencia utilizando un conversor digital-analógico de 12 bits de buenas prestaciones, como es el AD7945 de Analog Devices. Este conversor es multiplicador en cuatro cuadrantes respecto de la referencia (la salida es el resultado de la conversión multiplicado por el valor de la referencia), por lo que puede utilizarse la siguiente estrategia: generar con el microcontrolador la forma base en 12 bits a fondo de escala (utilizando el rango dinámico completo del conversor), y controlar su amplitud externamente a través de la entrada de referencia.

El conversor AD7945 (AD7547 ídem dual) está compuesto por un circuito R-2R altamente estable (Figura 73a) al que se alimenta con una $V_{R E F}$ externa (entrada multiplicadora). Su salida es en corriente, por lo que se utiliza un amplificador operacional dual AD712 para convertir a tensión y sumar la referencia. La configuración recomendada por el fabricante puede verse en la Figura 73b. Su resolución es de 12 bits, con precisión de medio bit menos significativo. El tiempo de establecimiento de la corriente de salida es de 1,5 us máximo, suficiente para la tasa máxima de actualización de $100 \mathrm{KHz}$. El error de ganancia es bajo, garantizando menos que un bit menos significativo. La operación puede ser bipolar lo que permite realizar la multiplicación en cuatro cuadrantes [Analog Devices AD7547 datasheet].
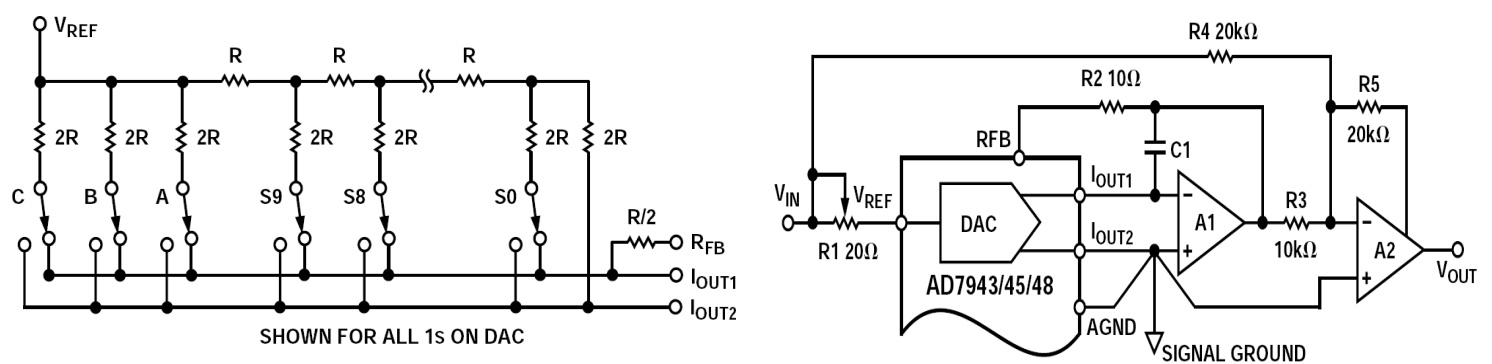

Figura 73: a) Diagrama simplificado de la red $R-2 R$ del conversor digital-analógico AD7945. b) Configuración para operación bipolar [Analog Devices datasheet].

El esquemático completo de una implementación autónoma, sin la interacción con ADuC, se presenta en la Figura 74. En este caso la frecuencia de operación y el GATE se configuran por medio de "jumpers" y para la $V_{R E F}$ se utiliza una tensión ajustable manualmente. En la versión integrada los dos primeros parámetros son programados por el procesador principal a través del bus interno, mientras que la tensión analógica se genera con las conversores DA integrados (ver a continuación). 


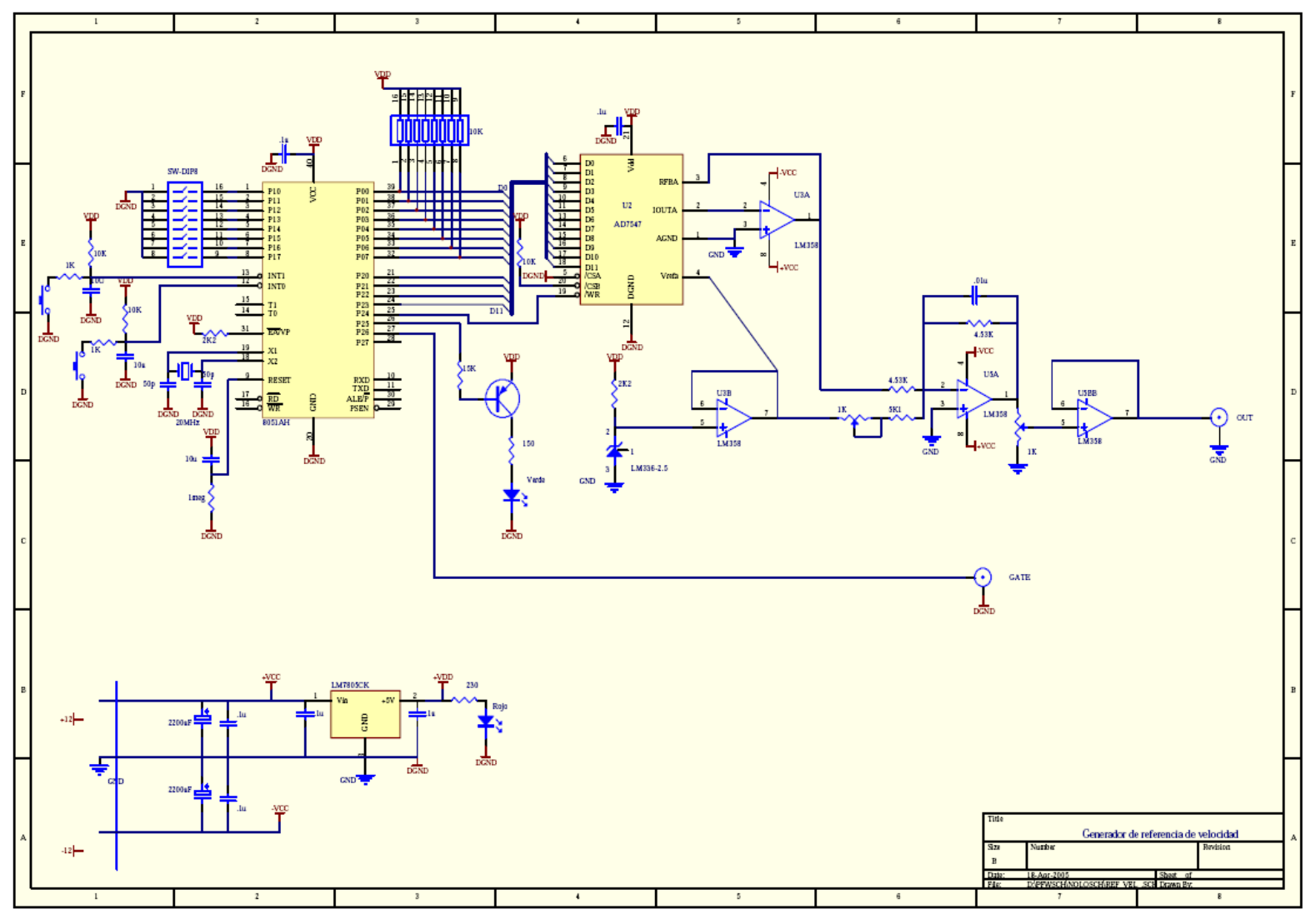

Figura 74: Implementación autónoma del generador de referencia digital de velocidad.

La programación del microcontrolador se realizó directamente en assembler, pues su operación es muy simple. Se utiliza uno de los timers para generar una interrupción a intervalos regulares (frecuencia de operación programable). Ante cada interrupción el microcontrolador obtiene un nuevo valor (en 12 bits) de una tabla, correspondiente a la amplitud de la referencia, envía el dato utilizando un port y medio de 8 bits P0.0 a P0.7 y P2.0 a P2.3, y acciona el /W del conversor con P2.4. Las tablas con las diferentes formas de referencia se generaron off-line utilizando Matlab y se almacenan en la memoria de programa del procesador auxiliar. Solo se almacena la forma del retorno ya que la zona plana se genera con un el otro timer/counter disponible, determinando el ciclo de trabajo.

En los $8 \mathrm{~KB}$ de memoria disponible pueden almacenarse hasta 4 retornos diferentes a 1000 puntos de 12 bits cada uno. Suponiendo la operación del transductor a $10 \mathrm{~Hz}$, con un ciclo de trabajo del 50\% (peor caso), resulta una frecuencia de muestreo del retorno de $20 \mathrm{KHz}, 3$ órdenes por encima de la frecuencia de operación.

El generador maneja tres señales digitales, que hacen al funcionamiento sincronizado con el procesador principal:

TRIGGER_OUT: indicación del centro del recorrido para habilitar el cambio de velocidad, salida en P2.5.

TRIGGER_IN: entrada a /IRQ1 del procesador auxiliar, preparado para recibir información de sincronización externa del sensor de posición discreto. 
GATE_OUT: señal en sincronismo con la referencia de velocidad que indica la zona plana, salida en P2.6.

\subsubsection{Anulación del valor medio}

Tal como se mencionó en repetidas oportunidades, debe garantizarse que el valor medio de la referencia de velocidad generada digitalmente sea nulo, a fin de no exigir los mecanismos de compensación del sistema de control. Dado el circuito de salida de la Figura 73b, el mecanismo de compensación de la tensión de offset del conjunto debe ser el siguiente:

i. La tensión de offset del primer amplificador operacional (A1) no afecta la salida, porque la entrada es en corriente.

ii. Con $V_{R E F}=0$ debe eliminarse el offset del amplificador de salida (A2) con su propia compensación.

iii. Para compensar el offset de la señal digital, debe generarse una salida poniendo un valor de tensión en $V_{R E F} \mathrm{y}$ con el potenciómetro $\mathrm{R}_{4}$ debe anularse el valor medio. Para esto conviene usar una frecuencia de operación alta y un voltímetro que mida valor medio. El offset de la digital no puede compensarse en el operacional de salida porque la señal puede invertirse ( $V_{R E F}$ bipolar) pero no lo hará la compensación de offset.

Para el circuito de la Figura 73b, sea $X$ es el valor de 12 bits digital a la entrada. La tensión de salida $V_{\text {OUT }}$ es proporcional a $V_{R E F}$ tal que la suma en el amplificador de salida vale:

$$
V_{\text {OUT }}=-\frac{R_{5}}{R_{3}}\left(-V_{R E F} \frac{X}{4096}\right)-\frac{R_{5}}{R_{4}} V_{R E F}
$$

Para los valores de la figura resulta:

$$
V_{\text {OUT }}=V_{R E F}\left(\frac{X-2048}{2048}\right)
$$

Cuando el conversor recibe $X=0 x F F F$ la salida vale $V_{R E F}$; cuando $X=0 x 800$ la salida vale 0 ; y cuando $X=0 x 000$ la salida vale $-V_{R E F}$.

Suponiendo una referencia digital que no tenga valor medio nulo, por ejemplo un seno A. $\operatorname{sen}(w t)+A_{0}$, la tensión de salida vale:

$$
V_{\text {OUT }}=2 V_{R E F}\left(\frac{A \operatorname{sen}(w t)+A_{0}}{4096}\right)-V_{R E F}
$$

Para que $V_{\text {OUT }}$ sea igual a $V_{R E F}$ la resistencia $R_{4}$ debe ajustarse para valer:

$$
R_{4}=\frac{20 \mathrm{k}}{\left(1+\frac{A_{0}}{4096}\right)}
$$

Cualquiera sea el signo de $A_{0}$. Si $V_{R E F}$ se invierte, la compensación sigue siendo válida. Esto implica que cada vez que se cambie la forma del retorno o se cambie el ciclo de trabajo (ambas afectan al valor medio de la señal digital) debe ajustarse $R_{4}$ con un instrumento de precisión. Almacenar todas las formas de retorno con el mismo valor medio atentaría contra 
un buen aprovechamiento del rango dinámico del conversor.

Por este motivo, la selección de la forma de onda y del ciclo de trabajo no puede hacerse de forma totalmente automática. Sin embargo, esto no representa una gran complicación ya que el procedimiento de sintonización del funcionamiento del transductor debe realizarse sólo una vez para cada espectrómetro. Debe incluir también el ajuste de la frecuencia de operación y la calibración del GATE, éstos si programables pues no afectan al valor medio. Es recomendable revisar periódicamente el funcionamiento del conjunto, a fin de detectar alteraciones de origen mecánico como partes flojas o rozamientos.

\subsubsection{Integración}

A continuación se presentan algunos detalles relacionados con la integración del generador con el procesador principal.

a) Bus interno de comunicaciones: 8 bits de datos y 2 de control (/IRQ0 sincronización y P2.7 selección). Se envían la frecuencia de operación o el tiempo de GATE en 8 bits. Estas dos variables no se almacenan en el procesador auxiliar, pues no dispone de memoria flash. El procesador principal los envía en el arranque o ante un cambio remoto.

b) La $V_{R E F}$ del conversor DA se obtiene como la resta de las salidas de los DA integrados en el procesador principal. Estos son conversores de 12 bits de regular calidad que utilizan la técnica de cadena de resistencias (ver Figura 75a). Presentan alinealidades en los extremos debido a la saturación del amplificador cuando opera con $V_{D D}$ como referencia (ver Figura 75b). Dadas las especificaciones para el rango, $V_{R E F}$ debe ser bipolar para obtener tanto velocidades positivas como negativas. La resta se realiza con el amplificador de instrumentación AD627. El DAC1 se utiliza para compensar el offset del $D A C 0$, a fin de garantizar la velocidad nula. El valor de $D A C O$ determina la velocidad de operación y el valor de $D A C 1$ pude configurarse remotamente y almacenarse. De este modo se minimiza el efecto de las alinealidades en los conversores, enviándolas a los extremos del rango. La calibración se realiza poniendo $D A C 0$ en $0 \times 800$ y moviendo $D A C 1$ hasta que se anule la diferencia en continua. Este procedimiento anula también el offset del AD627, no así el drift del mismo que vale 1 $\mathrm{uV} / \mathrm{C}$. Este valor es despreciable si se considera que 1 bit en el rango de $2,5 \mathrm{~V}$ representa $600 \mathrm{uV}$.

c) Señales digitales de sincronización entre los dos procesadores:

i. GATE_OUT: P2.6 del 8051 conectado a /INT0 e /INT1 del ADuC y disponible para monitoreo. Se utiliza para inhibir el conteo y la base de tiempo simultáneamente durante el retorno y tiempo de establecimiento.

ii. TRIGGER_OUT: P2.5 del 8051 conectado a P1.7 del ADuC. El procesador auxiliar indica el centro del recorrido, habilitando al ADuC para que aplique la nueva amplitud de la referencia. 

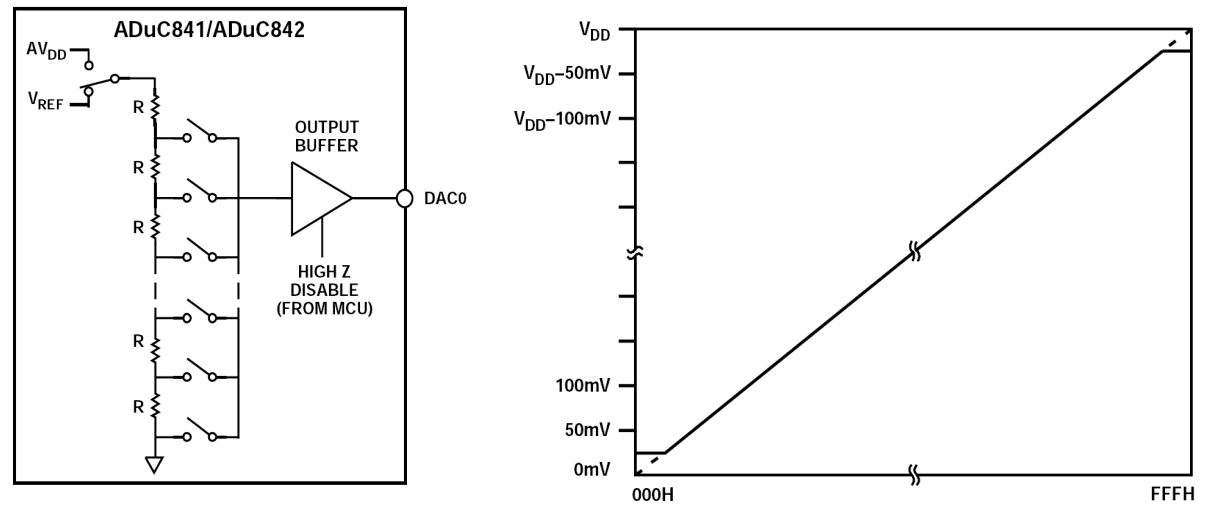

Figura 75: a) Modelo equivalente de la cadena de resistencias de uno de los conversores digital-analógico del ADuC841. b) Alinealidades en los extremos debido a la saturación del amplificador cuando opera con $V_{D D}$ como referencia [Analog Devices ADuC841 datasheet]

Ambos conversores tiene monotonicidad garantizada, siendo el error de linealidad del AD7945 menor que un bit menos significativo. La linealidad de los conversores del ADuC se verificará en el próximo capítulo a través de una calibración Mössbauer con un absorbente de referencia. Con la configuración multiplicadora se obtiene una resolución de 12 bits para todos los canales. La distorsión armónica es despreciable por operarse con 12 bits a una frecuencia mínima de muestreo de $20 \mathrm{KHz}$. Pueden utilizarse los 4096 canales teniendo en cuenta la saturación en los extremos.

\subsection{Operación en tiempo real e interfaz de usuario}

Como se mostró en la Figura 69 existen dos niveles diferenciados de comunicaciones. Se procuró resolver internamente todas las tareas cuyas restricciones de tiempo fueran importantes (operación en tiempo real), mientras que la interacción automática y la interfaz de usuario se realizó a través de un enlace serie de baja prioridad. Una vez configurado el instrumento, para la operación normal lo único que necesita transmitirse desde el exterior es cuánto tiempo debe contarse y en qué canal, mientras que el instrumento contesta con el número de eventos detectados en dichas condiciones. Se hace referencia a esta comunicación como de baja prioridad ya que un retardo inesperado en este enlace produce el efecto de aumentar el tiempo total que insume la medida, pero no deteriora la calidad de los resultados.

Se implementó el enlace a PC vía interfaz RS232 programada con la UART integrada del AduC841 funcionando con 8 bits de datos, 1 de stop, sin paridad a 115000 baudios, más un driver integrado (MAX232 de Maxim). Esta interfaz tiene la ventaja de ser fácilmente extendible a USB o Bluetooth, aunque la modificación no se consideró necesaria inicialmente.

Para la operación interna en tiempo real se utilizó el micro-kernel desarrollado para la Tesis de Magister. Se trata de una estructura macro que permite asociar múltiples tareas simples a diferentes interrupciones y ejecutarlas en un entorno preemptivo basado en prioridades. Para ello debió simularse un entorno de tantos niveles de prioridad como tareas a ejecutar, disponiéndose del sistema de interrupciones en dos niveles de la arquitectura 8051. Además 
permite disponer de tareas de background que en este caso se asociaron a las tareas de interfaz de usuario y comunicaciones sin restricciones de tiempo. Las tareas de tiempo real se utilizaron para resolver los requerimientos temporales estrictos, como son en este caso la sincronización entre las etapas, inhibición y conteo de eventos, base de tiempo, realimentación de posición, etc.

Para facilitar la automatización de los experimentos, se elaboró un juego de rutinas que interactúan en bajo nivel con el instrumento, presentando una interfaz de alto nivel al usuario. Para esta tarea se utilizó el lenguaje de programación Matlab de alto nivel, ya que dispone de una buena librería de interacción con la puerta serie, además de sus evidentes facilidades para manejar vectores y matrices. Este mecanismo permite la elaboración de sofisticados experimentos en un lenguaje de alto nivel bien conocido, con la facilidad de interactuar con el instrumento desde adentro del programa.

Las rutinas tienen una interfaz intuitiva y prevén los siguientes modos de operación:

i. Modo de velocidad programable nativo del instrumento.

ii. Modo de velocidad constante.

iii. Modo de aceleración constante emulado.

No se presentarán los detalles de programación por ser un tema que se encuentra fuera de los alcances de esta tesis. Tanto las rutinas como una especificación detallada del funcionamiento interno de los procesadores y el protocolo de comunicación se encuentran disponibles. En el capítulo siguiente se presentará la funcionalidad completa a través de un caso de aplicación dinámico que explota a fondo las capacidades del sistema.

Por último se mencionará un modo alternativo de funcionamiento que se programó en el procesador principal, explotando los beneficios de la velocidad programable. A través de la interfaz de usuario puede seleccionarse un canal de inicio, un canal final y un paso en canales. Con estas variables configuradas el equipo puede ser iniciado en un modo de barrido automático, sin que sea necesaria la interacción del usuario. Esta facilidad permite relevar zonas del espectro en forma automática sin necesidad de programación. Su utilidad se demostrará también en el próximo capítulo a través de un ejemplo.

\subsection{Implementación}

Todos los módulos descriptos en este capítulo se integraron en una placa única, la cual fue diseñada con el objetivo de ser montada en un módulo NIM de ancho simple. En la Figura 76 puede verse el módulo en su implementación final montado en un rack y en la Figura 77 se presenta el esquemático completo de la implementación integrada

Algunos aspectos adicionales de diseño se enumeran a continuación:

i. Se dispusieron dos potenciómetros multivuelta de fácil acceso desde el exterior para la compensación de offset de la referencia y uno más para el fondo de escala.

ii. Dos leds se montaron sobre el panel frontal, comandados uno por cada procesador, para realizar indicaciones visibles para el usuario.

iii. Se utilizaron reguladores de $-5 \mathrm{y}+5 \mathrm{~V}$, alimentados con la tensión de $12 \mathrm{~V}$ del rack, 
para no depender de la calidad de las alimentaciones disponibles.

iv. Se dispusieron alimentaciones y tierras independientes para los circuitos analógicos y digitales.

v. Para programación on-board de ambos micros, selección de entrada NIM o TTL y deshabilitación del GATE, se colocaron los “jumpers” y conectores necesarios.

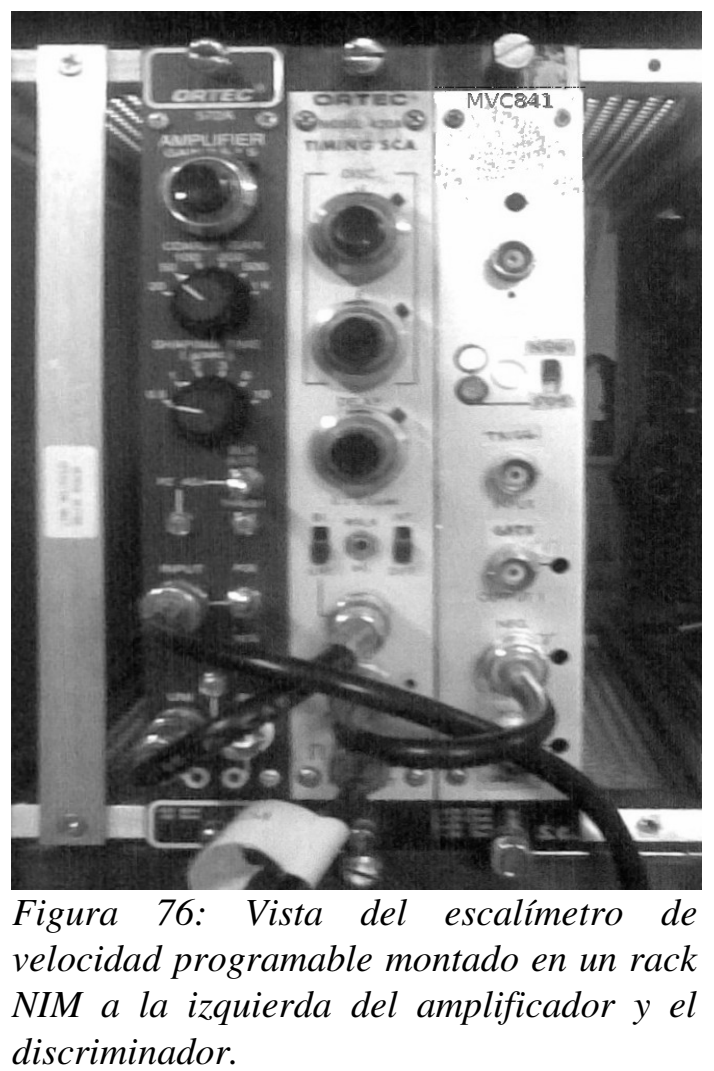

De este modo se completa la descripción de la instrumentación asociada a la etapa de procesamiento del espectrómetro de velocidad programable. Los esquemáticos, circuitos impresos, programas de los microcontroladores y la lista completa de componentes se encuentran disponibles. 


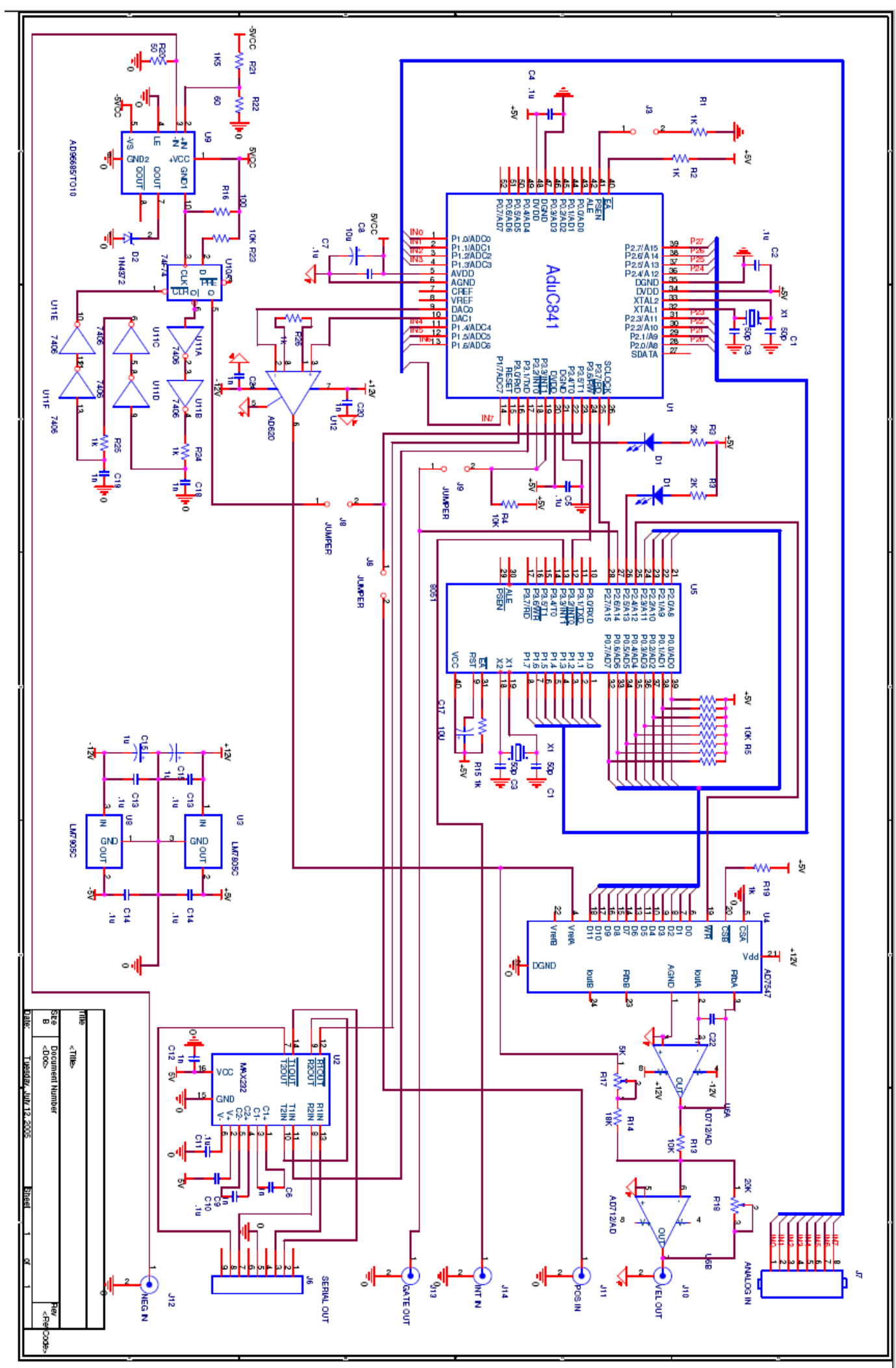

Figura 77: Esquemático single-board completo, incluyendo el hardware del generador, de acondicionamiento de pulsos y comunicaciones. 


\section{Capítulo 6 Aplicaciones y resultados}

En este capítulo se presentan algunas de las aplicaciones del nuevo espectrómetro de velocidad programable que han sido implementadas en laboratorio y han arrojado resultados interesantes. De ninguna manera intenta ser una enumeración exhaustiva de todas las posibles aplicaciones del instrumento. Sólo se mencionan las que hasta el momento han probado arrojar buenos resultados, dejando abierta la posibilidad de futuros experimentos que puedan diseñarse explotando la nueva funcionalidad.

Para ello se presenta primero una calibración experimental con hierro natural para el espectrómetro completo. Este procedimiento permite obtener varios parámetros que indican la calidad de las medidas que se realizarán luego. Luego se presentan los principales experimentos realizados poniendo especial atención en aquellos que no podrían realizarse con el equipamiento tradicional de laboratorio.

\subsection{Calibración}

Un método ampliamente aceptado para caracterizar las prestaciones de un espectrómetro Mössbauer consiste en obtener, en condiciones experimentales similares a las de medida, un espectro completo de absorción de una muestra de calibración bien conocida, en general de hierro natural. Este tipo de absorbente se encuentra perfectamente caracterizado y está siempre disponible en los laboratorios, ya que se utiliza para ajustar la abscisa de los espectros (procedimiento llamado de calibración).

A fin de estudiar el desempeño del nuevo espectrómetro, el espectro obtenido con la nueva técnica se comparará con un espectro obtenido con la técnica de aceleración constante tradicional en las mismas condiciones experimentales. Este procedimiento permitirá comparar 
los siguientes factores de calidad:

i. Eficiencia: Para un determinado tiempo de medida, la eficiencia estará determinada por la relación señal a ruido del espectro obtenido o, lo que es lo mismo, la profundidad de las lineas de absorción versus el ruido de fondo del espectro. Este parámetro se formalizó a lo largo del Capítulo 3. Debe tenerse en cuenta la reducción de la eficiencia implícita que acarrea utilizar la técnica de velocidad constante.

ii. Linealidad: Se conocen de antemano, con exactitud, las velocidades relativas a las que se encuentran las líneas de absorción de la muestra de calibración. Esto permitirá determinar la linealidad del instrumento en la abscisa del espectro, más precisamente en los seis puntos determinados por las singularidades del hierro.

iii. Resolución: La selectividad en energía del instrumento estará evidenciada en el ancho de las lineas de absorción del espectro, medido a mitad del máximo. Este es un parámetro de calidad estándar de los espectros Mössbauer, que permite comparar resultados producidos por diferentes configuraciones, pues hace referencia directa a la calidad del control de velocidad.

\subsubsection{Materiales y métodos}

Para ambas calibraciones se utilizaron los siguientes parámetros y componentes comunes:

i. Número de canales: 1024.

ii. Rango de velocidades: desde -8 a $+8 \mathrm{~mm} / \mathrm{s}$.

iii. Tiempo: 1 hora por espectro de calibración.

iv. Fuente: $\mathrm{Co}_{57}$.

v. Absorbente de calibración: lámina de hierro natural (natural iron foil) con espesor levemente mayor que el espesor óptimo (20 um). Este material produce un sextete (desdoblamiento magnético) con las líneas de absorción en las siguientes posiciones (en $\mathrm{mm} / \mathrm{s}$ ): $-5.4,-3.2,-0.9,0.7,3.0$ y 5.3. El centro del espectro (corrimiento isomérico) se encuentra en $-0.1 \mathrm{~mm} / \mathrm{s}$. Los anchos de línea varían entre 0.2 y 0.25 $\mathrm{mm} / \mathrm{s}$ y las profundidades relativas de las seis líneas son de 3, 2, 1, 1, 2 y 3 veces. El efecto (profundidad absoluta) depende del espesor de la muestra, del criterio de selección de eventos y otros parámetros.

vi. Etapa de detección: instrumental ORTEC optimizado según Capítulo 3 para máxima relación señal a ruido, por lo que debe esperarse al menos un efecto del $15 \%$. Detector tipo contador proporcional, preamplificador modelo $142 P C$, amplificador modelo $572 \mathrm{~A}$ y discriminador diferencial modelo $420 \mathrm{~A}$. Resultó una tasa óptima de $10 \mathrm{Kc} / \mathrm{s} \mathrm{a}$ la salida del discriminador.

vii. Etapa de accionamiento: Transductor CMTE, modelo MA-250, con su control de velocidad modelo $M R-350$.

viii.La estabilidad térmica del laboratorio se encuentra garantizada por un equipo de aire acondicionado que controla la temperatura de la habitación en modo on-off con un ripple máximo de 5 grados centígrados de amplitud y una frecuencia de 15 minutos. 
Para la etapa de procesamiento, en el caso de la calibración a velocidad programable se utilizó el nuevo escalímetro descripto en el Capítulo 5, operando en el modo de frecuencia constante a $12.5 \mathrm{~Hz}$, con retorno con forma de potencia cuarta del seno, ciclo de trabajo del $80 \%$ y eficiencia del $75 \%$. Se registraron 1024 velocidades igualmente espaciadas en todo el rango.

Esta etapa de procesamiento fue reemplazada para la calibración a aceleración constante por una placa para PC marca Nucleus modelo MCS II en modo de aceleración constante con 1024 canales (dwell-time de 50us). El resto de los componentes del espectrómetro se conservaron, con los mínimos ajustes necesarios, como por ejemplo una adecuada resintonización de los parámetros del control de velocidad que difieren para ambas técnicas.

\subsubsection{Resultados}

Los espectros obtenidos en ambos casos se ajustaron por los medios habituales. Los resultados se presentan en la siguiente tabla:

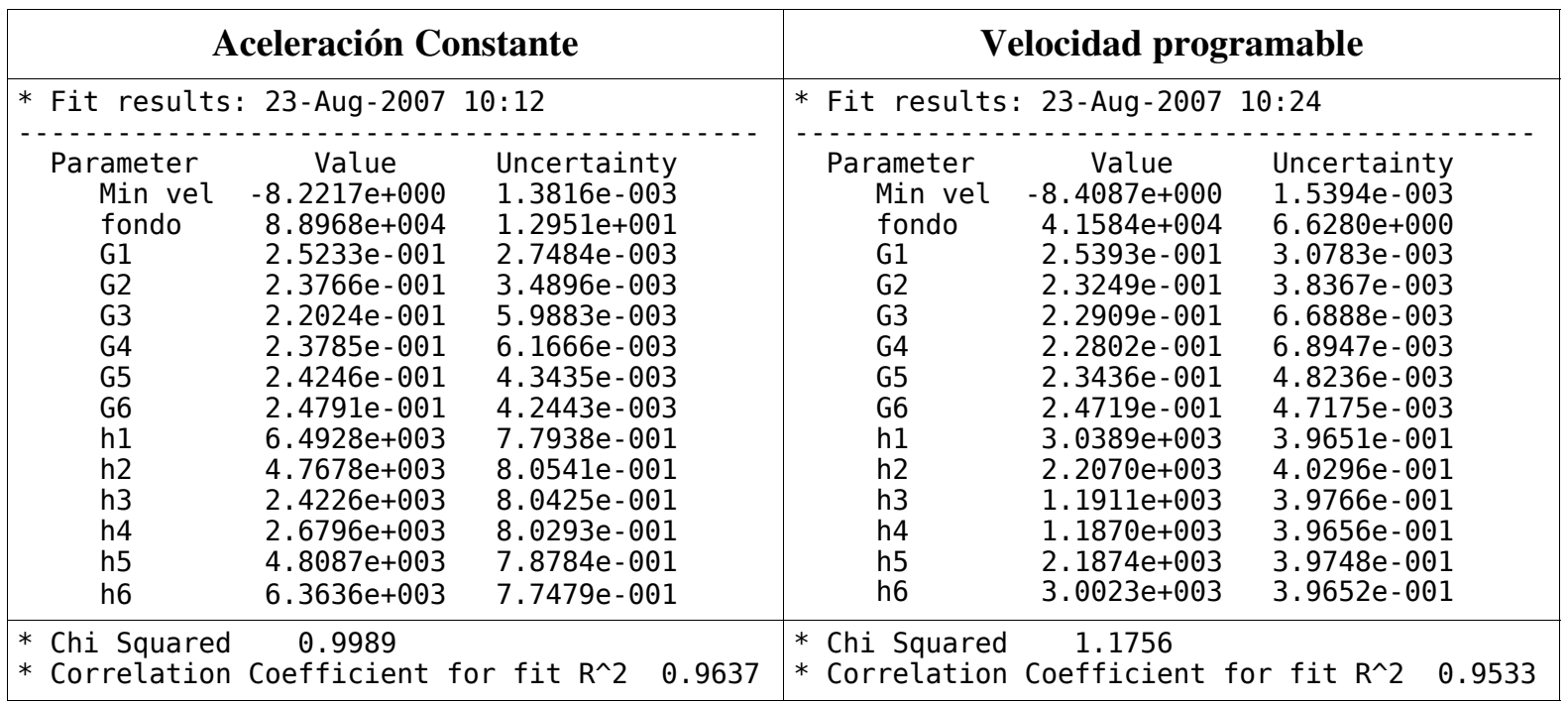

Debe notarse que el espectro de 1024 canales obtenido con la técnica de aceleración constante, luego del plegado, resulta en un espectro de 512 canales con el doble de estadística por canal. Eso explica la diferencia en el parámetro fondo de la tabla, además de que el modo de velocidad programable opera con un $75 \%$ de eficiencia. El espectro de velocidad programable tiene entonces el doble de puntos, pero la estadística es similar en ambos casos (una hora de medida). Por lo tanto los resultados son comparables.

En la Figura 78 pueden verse superpuestos los dos espectros normalizados. De la misma pueden extraerse las siguientes consideraciones. Respecto de la eficiencia, puede observarse que la relación señal a ruido es similar en ambos casos. Ninguno de los dos espectros es más ruidoso. La linealidad es excelente en ambos casos pues los canales correspondientes a los mínimos coinciden exactamente. Respecto de la resolución, puede verse que el ancho de línea es muy bueno en ambos casos (mejor que $0.21 \mathrm{~mm} / \mathrm{s}$ considerando el ancho de la muestra). No se puede decir que alguno de los dos sea más angosto, pero claramente el de velocidad programable es mas agudo. La estabilidad a largo plazo resultó satisfactoria tomando los 


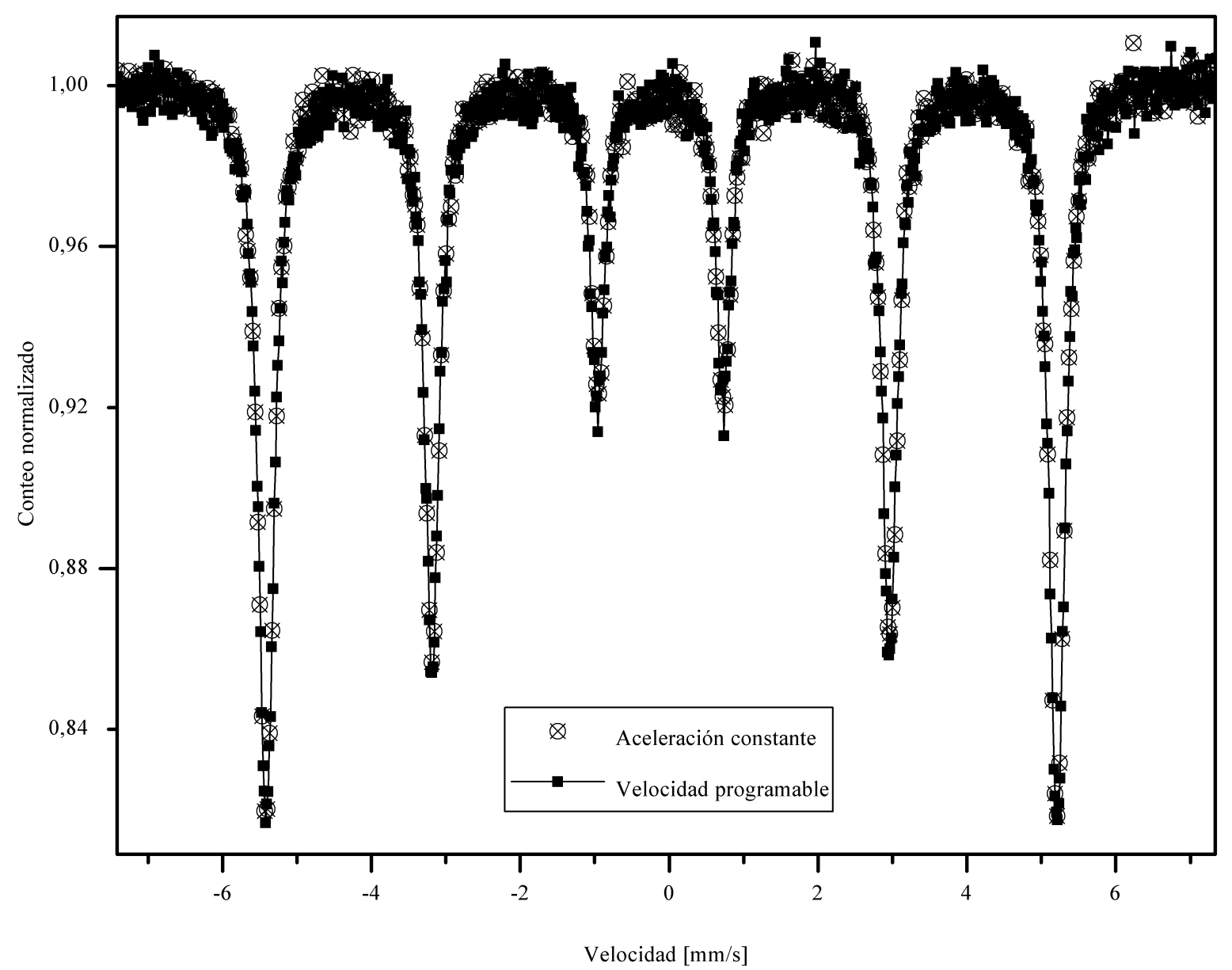

Figura 78: Espectros normalizados resultantes de las calibraciones. La abscisa representa la velocidad relativa en $\mathrm{mm} / \mathrm{s}$ luego del procedimiento de ajuste y la ordenada la absorción relativa.

recaudos correspondientes a la estabilidad térmica de los detectores. Una calibración en las mismas condiciones arrojó resultados idénticos al día siguiente.

Ambos espectros son muy similares y, en términos relativos, representan mediciones de muy buena calidad. Incluso la técnica de aceleración constante se vio beneficiada con la optimización de las etapas de detección y accionamiento. En el caso de la medición a velocidad programable puede verse que los mínimos son más profundos, resultando $18.24 \%$ versus $18.01 \%$ de efecto relativo (ver el detalle de la quinta línea en la Figura 79). Este es el resultado de la mayor resolución innata del método que controla con una constante (y no con una rampa entre canales, como el de aceleración constante). Al incrementarse la profundidad de las líneas se beneficia la relación señal a ruido del espectro, resultando en una compensación de la pérdida de eficiencia que origina el control de velocidad constante.

En conclusión, un espectrómetro de aceleración constante de muy buenas prestaciones fue mejorado en funcionalidad al introducir la técnica de velocidad programable. Esto se consiguió sin desmejorar aspectos importantes del experimento, como son la resolución, estabilidad, linealidad y eficiencia. Desde el punto de vista práctico, este resultado es suficiente para demostrar que el objetivo de la tesis fue logrado: obtener la operación 


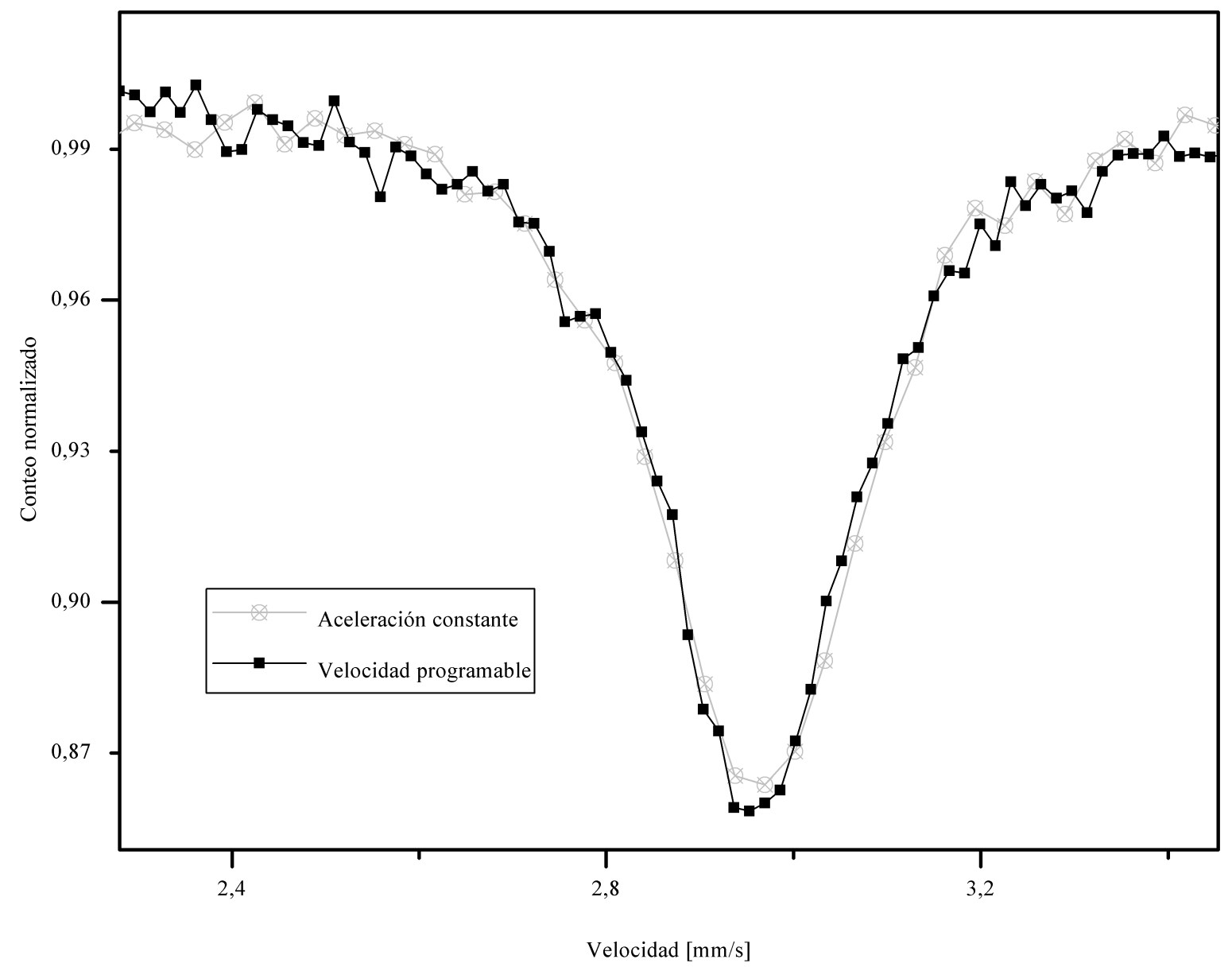

Figura 79: Detalle de la quinta línea de absorción de la figura anterior.

independiente canal por canal, basada en la técnica de velocidad constante, para conseguir los beneficios funcionales que la técnica implica. Esto significa registrar espectros parciales de muy buena calidad, introduciendo al laboratorio funcionalidad inexistente hasta el momento. En lo que resta del capítulo se presentará un conjunto de aplicaciones novedosas que tiene su origen en la nueva técnica.

\subsection{Primeras aplicaciones}

Las primeras aplicaciones de este instrumento que arrojaron buenos resultados fueron experiencias estáticas basadas en la técnica de velocidad constante. El espectrómetro de velocidad programable presentado tiene buena estabilidad a largo plazo en velocidad, por lo que es ideal para operar a velocidad constante. El modo programable es útil en estos casos para encontrar el punto de interés. Una vez seleccionada la velocidad de operación se trabaja en forma estática (medición en un solo canal).

Tal es el caso de la determinación de la dinámica de los momentos magnéticos del hierro [Pasquevich et al 2006] [Mendoza Zélis et al 2002]. En estos casos se opera a velocidad constante mientras que se produce un barrido del campo magnético aplicado a la muestra. Para estos experimentos se diseño instrumentación adicional que se presenta en el Apéndice 
B. Un caso de aplicación similar de velocidad constante es el estudio de dinámicas de cristalización de nanocompuestos [Saccone et al 2004].

Un caso más elaborado de aplicación es la determinación de la dependencia con la temperatura del campo magnético hiperfino [Mendoza Zélis et al 2002] [Pasquevich et al 2004] [Pasquevich et al 2006], o la evolución térmica de materiales metaestables [Mendoza Zélis et al 2004] [Sánchez et al 2004]. Estos casos de aplicación presentan la particularidad de requerir la medición de la absorción a dos o tres velocidades constantes diferentes, mientras se barre la temperatura de la muestra en estudio. Una forma de realizar el experimento es funcionando a velocidad constante y realizando el ciclado térmico tres veces, o bien utilizando la velocidad programable para obtener la absorción a tres velocidades durante un único ciclado térmico. Esta última opción es preferible porque evita tener que repetir el barrido en temperatura con precisión, pero sólo es posible con el espectrómetro de velocidad programable.

En base al éxito de estos experimentos, las técnicas de barridos térmicos y magnéticos empezaron a ser formalizadas en los últimos años, introduciendo una herramienta adicional al laboratorio de espectroscopia Mössbauer. Las técnicas de barrido cobran importancia cuando el tiempo disponible para realizar la medición es insuficiente para obtener un espectro útil por métodos tradicionales.

Si bien estas aplicaciones produjeron resultados muy interesantes, no sirven para evidenciar la real potencialidad del instrumento. Éstas podrían realizarse con un espectrómetro de velocidad constante bien calibrado en modo interactivo, aunque la operación sería tediosa. Por ese motivo se presentarán a continuación dos aplicaciones para el espectrómetro de velocidad programable que explotan a fondo su capacidad y además producen resultados que no pueden ser obtenidos con instrumental tradicional. La primera es una aplicación muy dinámica, en la que el espectro evoluciona a lo largo del experimento, mientras se registra, no ya una única velocidad, sino una zona de interés compuesta por varios canales. Este tipo de experimentos implica que la velocidad de operación debe variarse en forma automática durante el transcurso del experimento, poniendo especial énfasis en la eficiencia de la medida. La segunda aplicación muestra un caso en el cual se desea observar una zona reducida del espectro, a la cual se le desea volcar la mayor estadística posible.

\subsection{Seguimiento de linea}

En esta sección se muestra cómo se utilizó la técnica de velocidad programable como base para desarrollar una nueva metodología [Veiga 2008]. Ésta permite el seguimiento automático de una línea de absorción resonante mientras su ubicación en el espectro se modifica como consecuencia de la variación de un parámetro externo, en este caso la temperatura a la que se encuentra sometida la muestra. En términos más generales, esta metodología permite la redefinición continua de una región de energías de interés, utilizando la capacidad de medición en canales independientes disponible en el escalímetro de velocidad programable y comandado al instrumento con un algoritmo de control. Esta estrategia permite concentrar las medidas en zonas del espectro en las cuales la información es más abundante, pero que a su vez evolucionan con el tiempo. 
Para comprobar el desempeño se realizó el seguimiento de la sexta línea del espectro del $\mathrm{FeSn}_{2}$ desde temperatura ambiente hasta por encima de su temperatura de Néel. En la Figura 80a puede verse un diagrama en bloques de la configuración experimental de velocidad programable utilizada, cuya descripción corresponde exactamente a la utilizada en la sección de calibración.

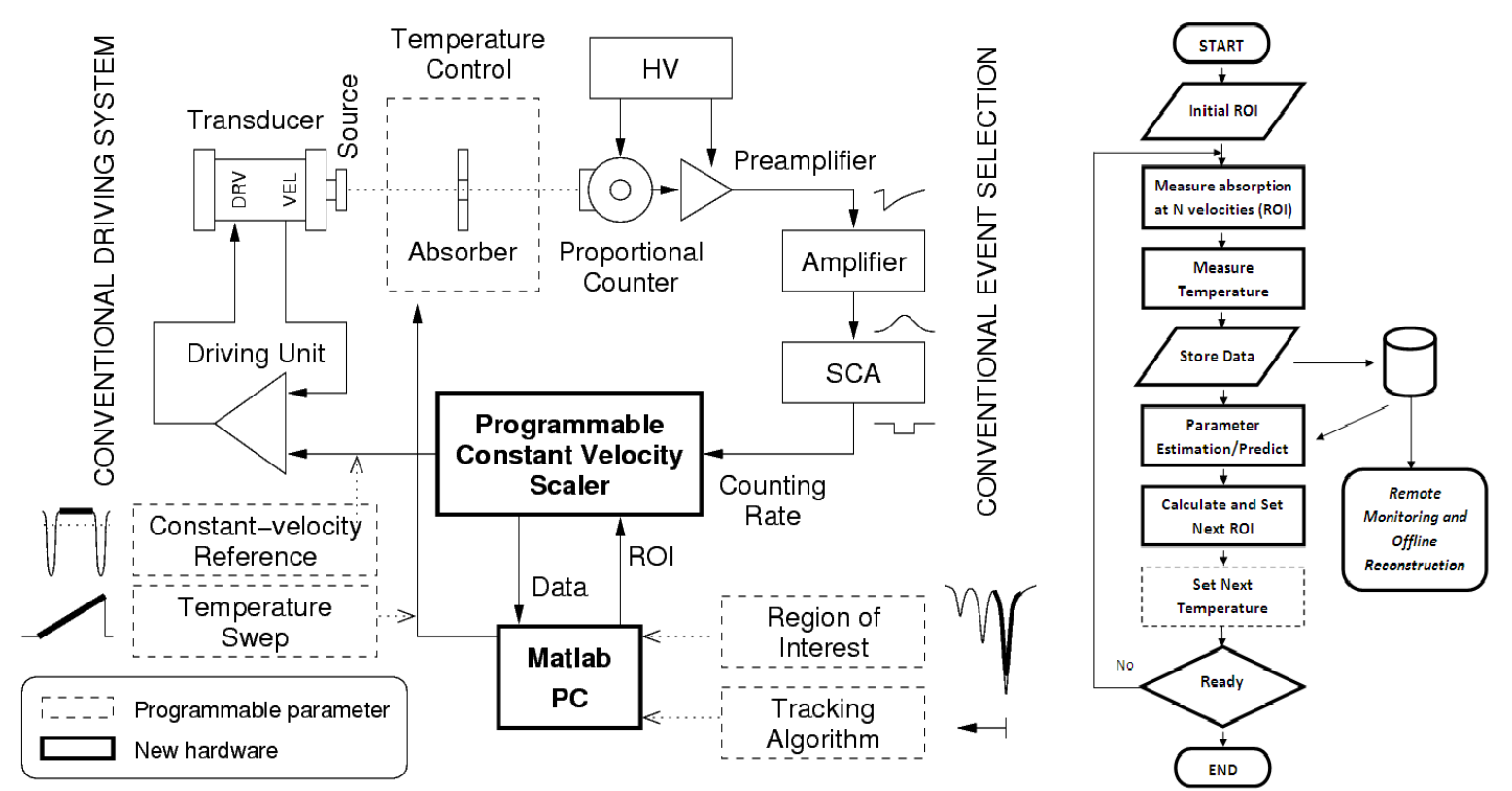

Figura 80: a) Configuración experimental para el seguimiento de linea, incluyendo los parámetros programables. b) Algoritmo de seguimiento ejecutado en la PC.

Para describir el seguimiento se utiliza una región de interés determinada por los siguientes parámetros:

i. $\quad N$ (entero): número de canales o velocidades diferentes a registrar.

ii. $\boldsymbol{C}(\mathrm{mm} / \mathrm{s})$ : centro de la región de interés.

iii. $\mathrm{S}(\mathrm{mm} / \mathrm{s})$ : espaciado entre canales.

iv. $\boldsymbol{t}$ (segundos): tiempo neto de conteo por canal.

El tiempo necesario para registrar una región de interés estará determinado por:

$$
T=N \frac{t}{e}
$$

Donde $\boldsymbol{e}$ es la eficiencia de operación a velocidad constante.

El diseño del experimento se completa con una estrategia de relocalización del centro de la región de interés. El caso interesante de analizar es el de seguimiento automático (a lazo cerrado), en el cual el nuevo centro se ubica en función de un parámetro extraído de la medición anterior de la región de interés (un mínimo en este caso).

Los parámetros iniciales de la región de interés deben ser seleccionados de acuerdo al 
conocimiento previo que se dispone acerca del material en estudio y en función de los requerimientos experimentales. Con estos parámetros iniciales, el algoritmo de control alojado en la PC (programado en Matlab en este caso) realiza el conteo en los $N$ canales durante el tiempo $t$ (Figura 1b). Utilizando los datos obtenidos e incluso su historia, el algoritmo calcula la posición esperada del centro de la nueva región de interés. Todos los datos obtenidos son almacenados para el análisis y reconstrucción posterior.

El tiempo $T$ impone un límite en la dinámica del experimento. El número de puntos no puede ser muy reducido ya que el ajuste puede no satisfacer el número de grados de libertad de la línea. Además el tiempo por canal debe ser suficiente para obtener una relación señal a ruido adecuada, parámetro que depende de la tasa y el efecto relativo de la línea. Existe entonces un compromiso entre la cantidad de estadística colectada y cuán rápido puede moverse la línea. Es importante notar la importancia que tiene en este punto el buen funcionamiento de la etapa de detección, por lo que se recomienda la utilización de la tasa óptima, un espesor adecuado de la muestra y un ventaneo eficiente.

El FeSn $\mathrm{S}_{2}$ es un antiferromagneto cuyo espectro consiste en un único sextete concentrado en el rango de $2 \mathrm{~mm} / \mathrm{s}$, que colapsa en un singlete a aproximadamente $70 \mathrm{~K}$ por encima de la temperatura ambiente, temperatura a la que ocurre la transformación de Néel $\left(T_{N}\right)$. El bajo valor de $T_{N}$ lo hace adecuado para experimentar con la nueva técnica, aunque el efecto es muy modesto: sólo 3\% en la sexta línea, si el espesor del absorbente está bastante por encima del espesor óptimo, lo que implica reducir considerablemente la tasa. Un buen balance se consiguió con una tasa de $6500 \mathrm{c} / \mathrm{s}$. Para obtener una aceptable relación señal a ruido en cada punto se seleccionó un tiempo por canal de 1.5 segundos. Si la eficiencia es del $75 \%$, una región de interés de 8 canales puede medirse en 16 segundos, lo cual proporciona una dinámica razonable. Si se permite que la línea se mueva sólo un $10 \%$ de su ancho en ese tiempo, resulta que el rango completo de $2 \mathrm{~mm} / \mathrm{s}$ puede barrerse en menos de media hora. Las simulaciones y resultados experimentales que siguen a continuación están centradas en este ejemplo.

Un aspecto interesante de este tipo de experiencias es que la temperatura de la muestra no necesita estar controlada para realizar el seguimiento. Sólo es necesario que pueda ser medida con exactitud mientras evoluciona naturalmente, por ejemplo, ante un escalón de potencia. La potencia aplicada debe ser tal que la derivada de temperatura no supere en ningún momento el límite expuesto en el párrafo anterior.

Se consideraron dos diferentes estrategias para el seguimiento de la línea. La primera consiste en una región de interés de múltiples puntos espaciados en forma de cubrir un rango de velocidades de dos veces el ancho de la línea; el algoritmo de seguimiento consiste en colocar el próximo centro en el mínimo actual. Este último se determina ajustando la región medida con una lorentziana.

La segunda estrategia es un caso interesante que contempla el mínimo número de canales que puede contener una región de interés (a fin de maximizar el tiempo por canal o aumentar la velocidad de barrido). Sólo dos puntos son necesarios para encontrar una lorentziana si se suponen conocidos e inalterables el fondo y el ancho de la línea. Los dos canales de la próxima región de interés se colocan aproximadamente en un tercio del ancho de la línea a cada lado del mínimo (puntos de máxima pendiente de la lorentziana). 
En ambos casos es posible agregar predicción al algoritmo por medio de la derivada primera de la posición del mínimo. En caso de ser necesario puede utilizarse un algoritmo más elaborado con parámetros variables.

Para sintonizar el diseño del experimento se utilizó un entorno de simulación compuesto por un conjunto de procedimientos para el entorno Matlab, en el que se puede observar el comportamiento dinámico del experimento y el efecto de variación de los parámetros. En la Figura 81 puede verse el resultado de una simulación para el caso del $\mathrm{FeSn}_{2}$ utilizando una región de interés multipunto mientras se produce un barrido de la temperatura entre ambiente y $380 \mathrm{~K}$ en menos de una hora. Se consiguió un seguimiento simulado satisfactorio utilizando los parámetros iniciales presentados anteriormente.

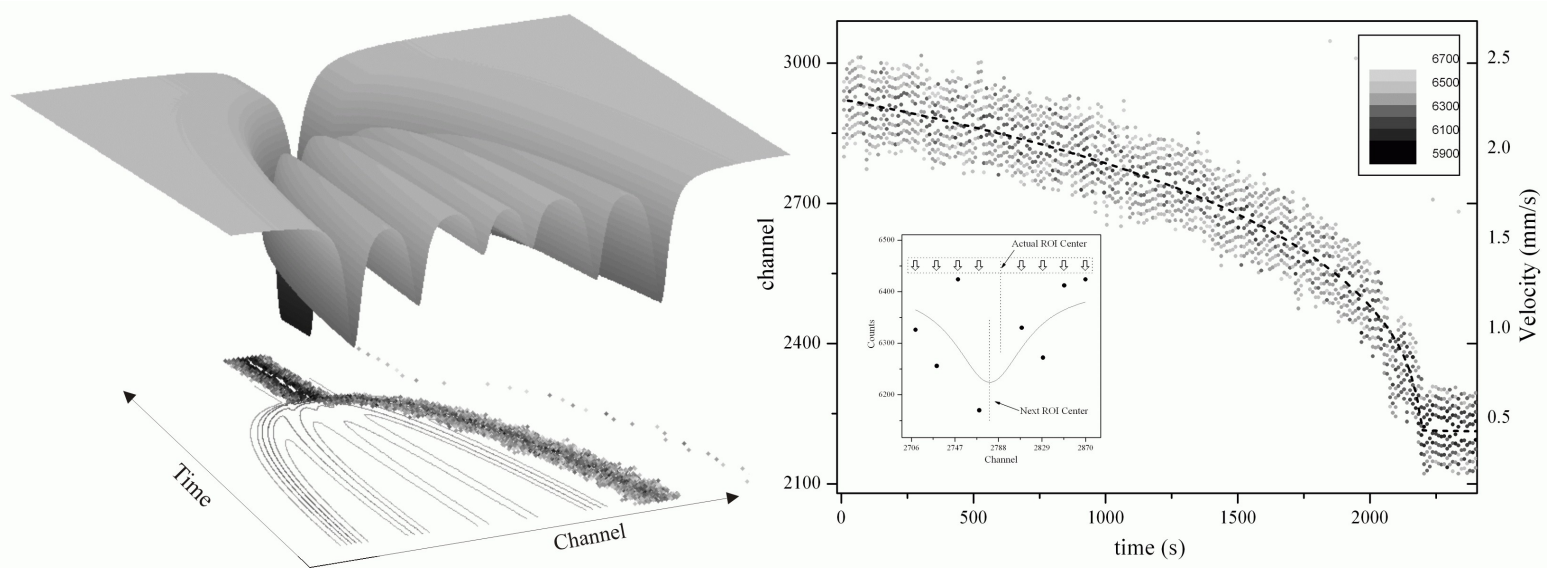

Figura 81: Simulación del seguimiento de la sexta línea del FeSn $n_{2}$ con una región de interés no uniforme de 8 canales, con una tasa de eventos de $6500 \mathrm{c} / \mathrm{s}$. a) Absorción en función del tiempo y la energía (canales). b) Evolución de la región de interés simulada en el tiempo, comparada con la posición del centro de la sexta línea (punteada); en el detalle se muestra el ajuste de una de las regiones de interés para la determinación del mínimo.

En la Figura 82 pueden verse tres instancias del experimento con fuentes de diferente actividad ( $2 \mathrm{mCi}$ y $50 \mathrm{mCi})$. En el primer caso debió utilizarse un barrido lento $\left(0.1^{\circ} \mathrm{C} / \mathrm{min}\right)$ y una región de sólo dos puntos. En el caso de la fuente de mayor actividad se pudo aplicar un barrido más rápido (del orden de $1{ }^{\circ} \mathrm{C} / \mathrm{min}$ ), pudiéndose contrastar la estrategia de dos puntos con una de ocho puntos. La sexta línea fue seguida exitosamente en todos los casos hasta producirse el colapso magnético, momento en el cual el algoritmo se engancha con la línea paramagnética. Una reconstrucción posterior con los datos registrados permitió determinar una $T_{N}$ de $95^{\circ} \mathrm{C}$ en todos los casos [Pasquevich a ser publicado].

Este tipo de experimento ha sido realizado para el estudio de otros materiales [Chien 1978] [Baek et al 1987] [Nama et al 2005], tomando espectros completos a algunas pocas temperaturas discretas, lo cual no sólo insume una cantidad de tiempo enorme, sino que además no permite disponer de información tan precisa acerca del comportamiento del material en la zona de interés. En base a los resultados puede afirmarse entonces que la metodología descripta presenta perspectivas interesantes para aplicaciones en las que se desea observar en forma detallada alguna región particular del espectro, incluyendo regiones que evolucionan en el tiempo. 


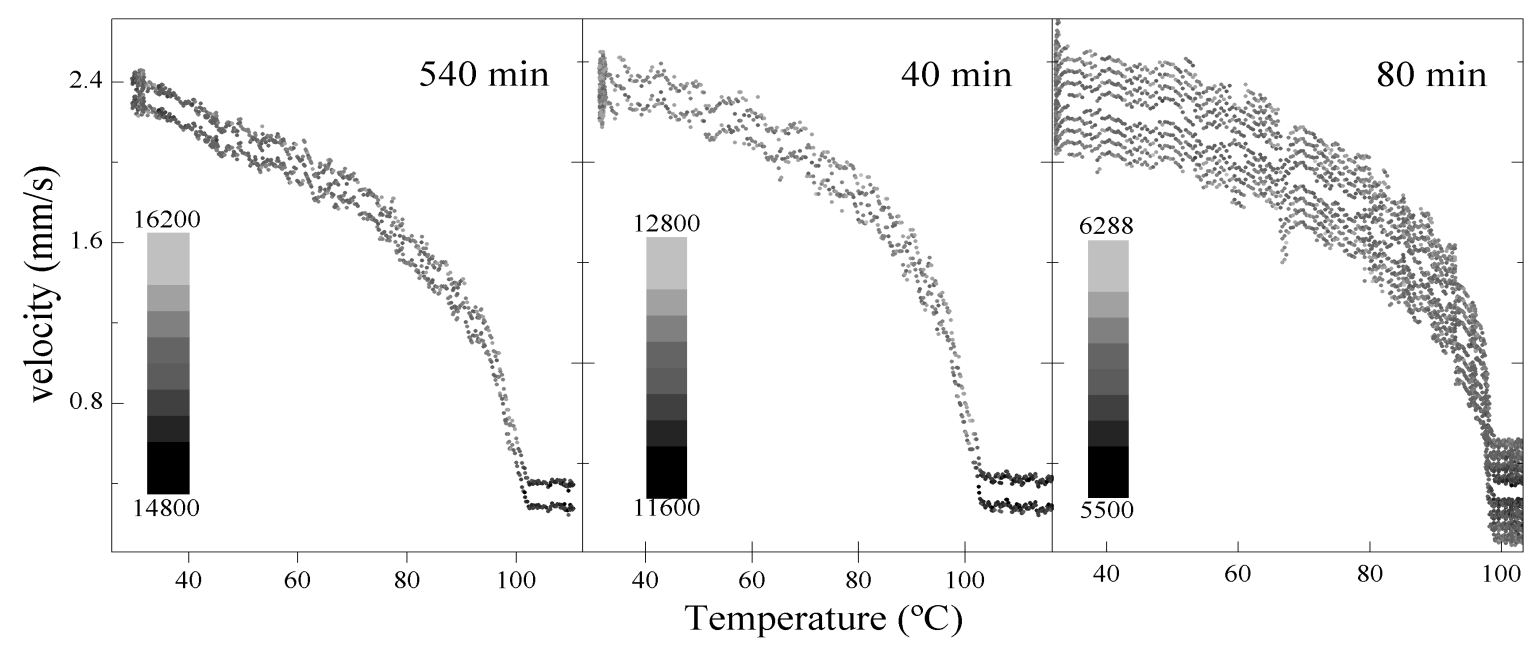

Figura 82: Resultados experimentales para el seguimiento de la sexta línea del FeSn2 (efecto de $2.5 \%$ ). a) Resultados utilizando una fuente de baja actividad $(2 \mathrm{mCi}, 850 \mathrm{c} / \mathrm{s})$ y una región de interés de 2 canales con $20 \mathrm{~s} / \mathrm{c}$. b) y c) Resultados obtenidos con una fuente más activa (50 $\mathrm{mCi}, 6500 \mathrm{c} / \mathrm{s}$, $100{ }^{\circ} \mathrm{C}$ por hora) utilizando una región de interés de 2 canales con $2 \mathrm{~s} / \mathrm{c}$ (b) y 8 canales con $1 \mathrm{~s} / \mathrm{c}$ (c).

\subsection{Magnificación selectiva}

Se describe a continuación un experimento simple pero contundente respecto de las ventajas de la selectividad disponible sobre el eje de energías.

Se dispone de una muestra de un compuesto de hierro que presenta desdoblamiento cuadrupolar con $3 \%$ de efecto aproximadamente (doblete en $+-1 \mathrm{~mm} / \mathrm{s}$ ). Se sospecha que la muestra puede contener una baja proporción de hierro en forma magnética (hematita), cuyo espectro de absorción es conocido (sextete con la primera línea en $-8 \mathrm{~mm} / \mathrm{s}$ aproximadamente). Se desea realizar la comprobación de la existencia de hematita utilizando espectroscopia Mössbauer. La baja concentración hace esperar un efecto menor que el $0.1 \%$ en la línea de mayor absorción del sextete, la cual se encuentra lejos del origen del espectro.

Para poder determinar la existencia de la línea, es necesario que el ruido estadístico en el fondo del espectro medido sea al menos un orden menor que la línea, o sea del $0.01 \%$. Para ello es necesario que el espectro acumule al menos $M$ cuentas por canal, estando $M$ determinada por:

$$
\frac{\sqrt{M}}{M}<\frac{0.01}{100}
$$

O sea que se necesitan al menos $10^{8}$ cuentas por canal. Si se dispone de una tasa de $10^{4} \mathrm{c} / \mathrm{s} \mathrm{y}$ se desea tomar un espectro utilizando la técnica de aceleración constante en 256 canales (es necesario pues debe medirse entre $-8 \mathrm{y}+8 \mathrm{~mm} / \mathrm{s}$ ), la medida insumiría casi un mes de laboratorio.

La determinación pudo realizarse en sólo un día utilizando el espectrómetro de velocidad constante. Se registró una región de interés de 5 canales igualmente espaciados, centrada en la 
posición en que debería estar la primera línea, más un punto alejado $(-10 \mathrm{~mm} / \mathrm{s})$ para registrar el fondo.

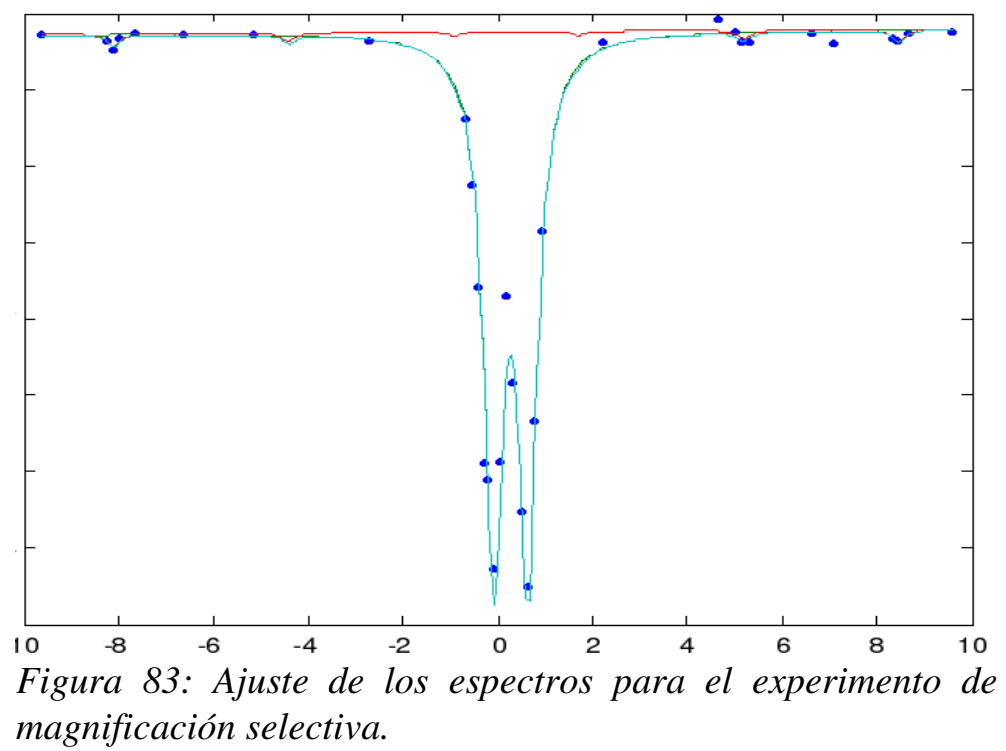

El experimento se repitió con dos zonas de interés registrando las líneas 1 y 2, lo que permitió ajustar un espectro completo y calcular así la concentración relativa de hematita en el compuesto, que resultó ser del 3.4\%. En la Figura 83 se muestra el ajuste de ambos espectros para un grupo seleccionado de puntos que permite calcular la concentración. 



\section{Capítulo 7}

\section{Conclusiones, aportes originales y líneas abiertas de trabajo}

En esta tesis se han presentado los resultados obtenidos a lo largo del diseño, implementación y verificación de una nueva técnica de espectroscopia Mössbauer con características propias. En este último capítulo se realiza una discusión final, resaltando las contribuciones personales realizadas y presentando las perspectivas de futuras investigaciones.

Respecto del núcleo central de la tesis, se ha mostrado cómo, al perfeccionar la operación a velocidad constante al punto de hacerla programable, no sólo se obtuvo un instrumento más versátil que sus predecesores sino que también se dio lugar a un nuevo modo de operación. Este nuevo modo, nombrado de velocidad programable, mejora en algunos aspectos las prestaciones de las técnicas Mössbauer conocidas y además agrega al laboratorio una funcionalidad hasta el momento inexistente, habilitando la realización eficiente de experimentos dinámicos sin precedentes.

Sin embargo, por estar basado en la operación a velocidad constante, el nuevo modo tiene la desventaja inicial de ver disminuida su eficiencia en la detección. Para salvar este inconveniente se dedicó especial atención a tres aspectos críticos del experimento: la optimización de la eficiencia en la detección y selección de eventos, el perfeccionamiento de la operación a velocidad constante y la optimización del sistema de adquisición y procesamiento de datos.

El primero de los temas condujo a un estudio detallado de los componentes electrónicos que integran la etapa de detección del experimento. La optimización de los dispositivos en conjunto permitió desarrollar un mecanismo novedoso que permite encontrar el criterio de ventaneo y la tasa de trabajo que hacen máxima la relación señal a ruido de los espectros. Se 
consigue así compensar en parte la pérdida de eficiencia inicial.

Respecto de la operación a velocidad constante programable, puede decirse que hasta el momento de inicio de esta tesis se trataba de un tema escasamente desarrollado. Luego de haber sido estudiados los transductores y los mecanismos de control en profundidad, se obtuvieron algunos aspectos sensibles de mejoras significativas que permitieron reducir el tiempo de retorno, minimizando así la pérdida de eficiencia. Los aportes más importantes en este sentido fueron la compensación del lazo de control, la optimización de la trayectoria de retorno, el procedimiento para el cambio de canal y la realimentación de posición.

La etapa de procesamiento del experimento fue rediseñada en su totalidad para cumplir con dos objetivos principales. El primero de ellos es coordinar la detección y el accionamiento, lo que implica una sincronización adecuada, una base de tiempo precisa y un tiempo muerto en el conteo despreciable. El segundo objetivo es satisfacer los requerimientos propios de un experimento moderno, como son la capacidad de programación y automatización, el acceso remoto y una interfaz de usuario adecuada.

El espectrómetro fue implementado en su totalidad y puesto en marcha en el Departamento de Física de la UNLP, encontrándose operativo actualmente. Los procedimientos de calibración realizados arrojaron resultados satisfactorios que garantizan la calidad de las medidas que se llevaron a cabo a continuación. Explotando las nuevas prestaciones se pudieron implementar experimentos novedosos que permiten tener una visión optimista respecto del futuro del nuevo instrumento.

En resumen, puede decirse que se consiguió liberar al espectrómetro Mössbauer de algunas de las serias limitaciones impuestas por la técnica de aceleración constante. Esta técnica obliga a medir espectros de rango continuo centrados en velocidad nula. En cambio, la nueva técnica de velocidad programable permite adquirir cada canal en forma independiente, concediendo mayor libertad a los espectros obtenidos.

Esto invita a proponer un nuevo punto de vista respecto de la resolución. Puede ahora plantearse cuál sería el número de canales necesarios para medir un determinado espectro. ¿Sería preferible volcar la estadística en unos pocos canales, ahora que es posible hacerlo? ¿O resulta mejor medir en un mayor número de canales con menor estadística? Esta cuestión no se encuentra presente en los espectrómetros de aceleración constante, pues su referencia de velocidad es una rampa y por lo tanto al disminuir el número de canales desmejora la medida. Cada canal acumula los eventos correspondientes a una zona de velocidad. En cambio, en el modo de velocidad programable la referencia es una constante en cada instante. Por lo tanto el tiempo de medida por canal es proporcional a la relación $\mathrm{S} / \mathrm{N}$ que se obtiene. En ese sentido, disminuir el número de canales puede mejorar el ajuste del espectro. Este razonamiento tiene un límite inferior en el número de puntos, el cual puede suponerse determinado por la cantidad de grados de libertad del ajuste posterior. Evidentemente, no será necesaria la misma cantidad de canales para medir un espectro totalmente desconocido que para saber cuánto se movió una línea conocida previamente.

Si se opta por adquirir un número reducido de canales, será preferible que estos se encuentren en una zona relevante del espectro, la que en general incluirá una o más líneas de absorción. En términos generales, puede aceptarse que la información del espectro se encuentra más concentrada en las líneas, mientras que el fondo no contiene tanta información. Incluso puede 
afirmarse que la línea contiene más información en las zonas de mayor pendiente. Medir más tiempo en esas zonas conduce a una reconstrucción más precisa del espectro. Pero para que la selectividad resulte útil debe disponerse de información previa respecto de lo que se está midiendo. Si, por ejemplo, se desconoce la posición de las líneas, se necesitará realizar el barrido de un gran número de canales para encontrarlas, no produciéndose mejora.

Este concepto queda bien ilustrado con el experimento de seguimiento de una línea presentado en la Sección 6.3. En cada ciclo se dispone de abundante información respecto de la línea de absorción, pues fue medida en sucesivos períodos anteriores. Incluso se sabe la dirección en que se está moviendo. Sólo se desea conocer la magnitud del desplazamiento y el cambio en el efecto al aumentar la temperatura. Este es un caso extremo en el que hay sólo dos grados de libertad, por lo que puede obtenerse una solución midiendo sólo dos canales. Este razonamiento es análogo al que fundamenta las experiencias de velocidad constante presentadas en la Sección 6.2, en las cuales se registran espectros de un solo punto que permiten obtener únicamente una variable.

Este concepto tiene actualmente su formalización teórica en proceso [Sánchez et al 2004] [Pasquevich et al 2006]. Sin embargo, es importante notar que en la práctica la técnica ha probado su eficacia. Se mostraron casos en los que fue posible reducir el número de canales a tal punto de registrar información significativa lo suficientemente rápido como para detectar cambios continuos en un espectro de absorción. Es tarea del experimentador el diseñar correctamente el experimento, pues cada caso de aplicación tiene sus características propias. Basándose en el tiempo disponible, los grados de libertad del espectro, y las características del material en estudio, el profesional debe diseñar la dinámica del experimento a fin de optimizar los recursos disponibles.

\section{Líneas abiertas de investigación}

Futuras investigaciones sobre los temas que siguen a continuación podrían conducir a un refinamiento de la técnica y a mejoras importantes en las prestaciones del instrumento.

\section{a) Digitalización de la etapa de detección}

Se propone diseñar un discriminador diferencial digital que muestree directamente la salida del preamplificador y genere a su salida un pulso lógico si detecta un salto de tensión de determinada altura. En este caso no sería necesario derivar en forma analógica ya que ello puede hacerse en forma digital, sin necesidad de conformar el pulso. La principal ventaja de esta propuesta es que se podría prescindir del amplificador, que es el instrumento más costoso del laboratorio. La etapa sería difícil de ventanear, pero puede utilizarse un amplificador sólo para observaciones, o bien puede realizarse el ventaneo utilizando técnicas digitales. No es posible muestrear directamente a la salida del detector porque su salida es en carga. El preamplificador es necesario para realizar la conversión a tensión.

Respecto de la velocidad de muestreo, debe considerarse que la salida del preamplificador está compuesta por exponenciales superpuestas con constantes de tiempo de aproximadamente 50 us. Para que la exponencial caiga menos del $1 \%$ entre muestras es necesario muestrear cada 0.5 us, lo que equivale a 2 Msamples/s. Si se desea menos que el $0.1 \%$ de error se necesita al menos 20 Msamples/s. 
Respecto de la resolución, debe tenerse en cuenta que el rango de salida del preamplificador es actualmente menor a un volt. Deberá trabajarse sobre su ganancia para reducir la incidencia del ruido eléctrico. Además debe atenderse el rango dinámico de entrada ya que las exponenciales de salida están superpuestas. Con 16 bits de resolución para el conversor debería ser posible resolver el problema.

Un procesamiento de señales con una resolución de 16 bits y una frecuencia de muestreo entre 2 y $20 \mathrm{MHz}$ es un desafío interesante, que cae dentro el ámbito de aplicación de los procesadores digitales de señales (DSP). En caso de resolverse exitosamente puede significar una disminución importante en el costo y un aumento en las prestaciones de la etapa de detección. El procesamiento digital de la señal debería reducir el tiempo muerto de conformación, que es el limitante actual en la tasa de trabajo.

\section{b) Utilización del tiempo de retorno del eje}

En la implementación actual, el tiempo de regreso del eje del transductor es un tiempo perdido respecto de la estadística, lo que se manifiesta en una eficiencia disminuida. Los eventos detectados durante dicho intervalo son descartados y no contribuyen a la medida. Podría implementarse un segundo ratímetro que mida la tasa durante este tiempo de retorno a mayor velocidad. Éste podría incluirse sin mayores complicaciones dentro de las prestaciones del escalímetro, más allá del significado físico que tenga dicha tasa.

Existen algunos casos en los que puede ser útil disponer de esa información. Por ejemplo, supóngase un ciclo de trabajo del $80 \%$. En este caso, cada cuatro ciclos de accionamiento puedo enviar una tasa equivalente compuesta por los eventos detectados durante los cuatro retornos. Si la velocidad a la que se está midiendo es lo suficientemente alta como para que no haya absorción a cuatro veces la velocidad en sentido contrario, lo que se estaría midiendo con el segundo ratímetro sería aproximadamente el fondo. Por ejemplo, en el caso del hierro natural, este criterio sería aplicable en el caso de estar midiendo la sexta línea de absorción del hierro $(5.3 \mathrm{~mm} / \mathrm{s})$; se sabe que a cuatro veces dicha energía del otro lado del eje (-21.2 $\mathrm{mm} / \mathrm{s})$ se estará lo suficientemente lejos de la primera línea $(-5.4 \mathrm{~mm} / \mathrm{s})$ como para afirmar que se trata de una medición del fondo. Sin embargo este criterio no aplica a la medición de las líneas 3 y 4 (-0.9 y $0.7 \mathrm{~mm} / \mathrm{s}$ respectivamente). En ese caso, el retorno se hace a una velocidad que corresponde con la posición de las líneas 5 y 2 respectivamente.

En las condiciones favorables, el escalímetro estaría en condiciones de medir directamente absorción relativa, lo cual aumentaría notablemente la eficiencia. Considérese el caso del experimento de magnificación selectiva presentado en la Sección 6.4, en el cual se midieron 5 canales sobre la posición esperada de la línea, más un canal de fondo para calcular el efecto. En caso de disponerse del segundo ratímetro no sería necesario medir en este último canal, lo que implicaría un aumento del $20 \%$ en la eficiencia.

\section{c) Etapa de accionamiento piezoeléctrica}

Los accionadores piezoeléctricos pueden ser una opción interesante para el accionamiento a velocidad constante. Dadas las características mecánicas de los mismos, difícilmente podría obtenerse un desplazamiento mayor que un milímetro, lo que aumentaría la frecuencia de operación, modificando las restricciones de la etapa de procesamiento. Estos dispositivos tienen la ventaja de ser pequeños, potentes, disponen de un buen ancho de banda y permiten 
posicionar en el orden de los nanometros. Entre las desventajas puede mencionarse que son costosos, deben operarse con tensiones altas, del orden de los KV y que los desplazamientos que se consiguen son muy limitados.

d) Software

Si bien no modificaría la calidad final de las medidas, un paquete de software amigable, intuitivo y portable liberaría al experimentalista de los aspectos técnicos de operación del instrumento, para permitirle concentrar su atención en los aspectos relacionados con la física del experimento. El entorno de trabajo podría confeccionarse inicialmente utilizando las facilidades interactivas de Matlab, aprovechando que ya han sido implementadas las rutinas de acceso al dispositivo en bajo nivel. Esta primer versión podría utilizarse para definir lo que debería llegar a ser una interfaz genérica para un experimento de velocidad programable, que contemple todas las posibilidades de aplicación sin complicar en exceso la operación. La versión definitiva debería programarse en un lenguaje eficiente y portable, respetando la filosofía open-source que caracteriza a todos los trabajos realizados en nuestros laboratorios. 


\section{Apéndice A}

\section{Mejoras en la etapa de detección}

El siguiente circuito discriminador diferencial puede incorporarse a la entrada del contador a fin de realizar la tarea de selección de eventos por altura del pulso. De esta manera puede prescindirse del discriminador NIM utilizado en la etapa de detección. El diagrama esquemático se presenta en la Figura 84.

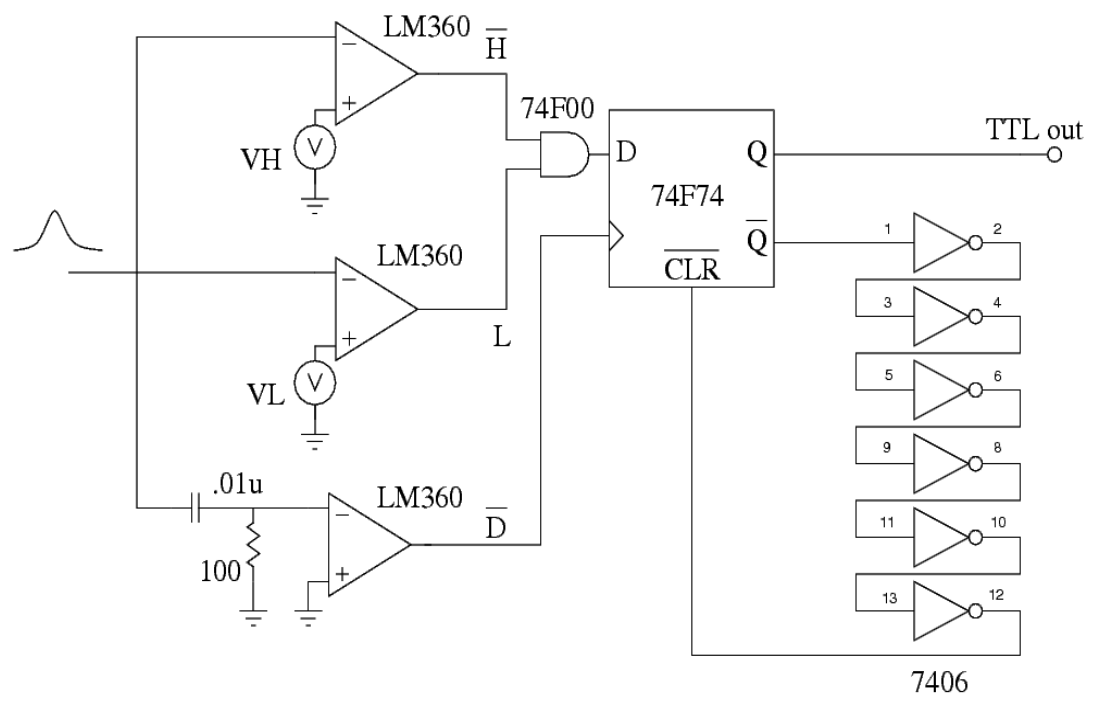

Figura 84: Diagrama esquemático del discriminador diferencial integrado.

Para su implementación se utilizan tres comparadores de alta velocidad LM360 de National (3 ns) con salidas complementarias para obtener las siguientes señales lógicas:

i. El nivel alto no ha sido superado $(/ \mathrm{H})$

ii. El nivel bajo ha sido superado (L)

iii. La derivada tiene valor nulo (/D)

Cuando la derivada del pulso de entrada vale cero (máximo) se activa el reloj del flip-flop. Si el nivel del pulso de entrada está en la ventana (/H AND L) el valor de la salida Q pasa a 1 y / Q a 0. Esto último produce un reset luego del retardo producido por las seis compuertas 7406 .

El monoestable ya se encuentra implementado en el diseño integrado (74F74 y retardo, ver Sección 5.2). Sólo se necesitan incorporar los tres comparadores y una compuerta 74F00. Para $V_{H}$ y $V_{L}$ pueden utilizarse dos helipot conectados a $V_{D D}$.

Este circuito se presenta como de implementación opcional ya que en los laboratorios de espectroscopia nuclear es muy común disponer de discriminadores de ventana que son operados con destreza por los físicos experimentales. Se recomienda su implementación si se desea simplificar la configuración del laboratorio o para una implementación de bajo costo.

Al circuito de la Figura 84 puede agregársele sin mayores inconvenientes un 
microcontrolador con dos conversores DA integrados (como el ADuC utilizado anteriormente, por ejemplo) a fin de comandar digitalmente los niveles alto y bajo de la ventana (por ejemplo via RS-232 o USB).

La nueva configuración programable puede utilizarse, utilizando el algoritmo adecuado y monitoreando el conteo, para realizar un ventaneo automático. Un algoritmo inteligente puede utilizarse para que la ventana se ajuste automáticamente ante un cambio en la sensibilidad del detector, lo cual es un problema como se mostró anteriormente, sobre todo considerando la dependencia de la ganancia con la temperatura. Esta estrategia resulta viable y de calidad aceptable, ya que un conversor DA de 12 bits, con sus 4096 niveles diferentes de tensión, tiene mejor resolución que un Helipot que tiene 2000 niveles diferenciables (correspondientes a 100 divisiones, 10 vueltas y un factor dos correspondiente a la media división. 


\section{Apéndice B \\ Barridos de campo magnético}

A continuación se presenta la instrumentación adicional necesaria para implementar una aplicación novedosa de la técnica mejorada de velocidad constante, publicada oportunamente [Pasquevich et al 2006]. En esta configuración la velocidad de la fuente se fija en una energía de interés, mientras se produce un barrido del campo magnético al que está sometido el material en estudio. Para el control del campo magnético se utilizó una disposición Helmholtz de bobinas (para obtener una zona de campo uniforme), cuya corriente se controló por medio de un amplificador especialmente diseñado a tal efecto. En la Figura 85 puede verse la configuración experimental.

Los bloques de instrumentación involucrados son los siguientes:

i. Rama de accionamiento compuesta por una implementación autónoma del generador de referencia de velocidad constante, más un transductor Wissel y su correspondiente control de velocidad.

ii. Rama de detección estándar (contador proporcional, preamplificador, amplificador de conformación y discriminador).

iii. Rama de procesamiento compuesta por un multiescalímetro Nucleus MCS II con el GATE del generador de referencia inhibiendo la entrada del contador. La onda triangular generada por el multiescalímetro se utilizó para barrer el campo magnético, para lo cual se diseñó un amplificador que acciona sobre la corriente de las bobinas.

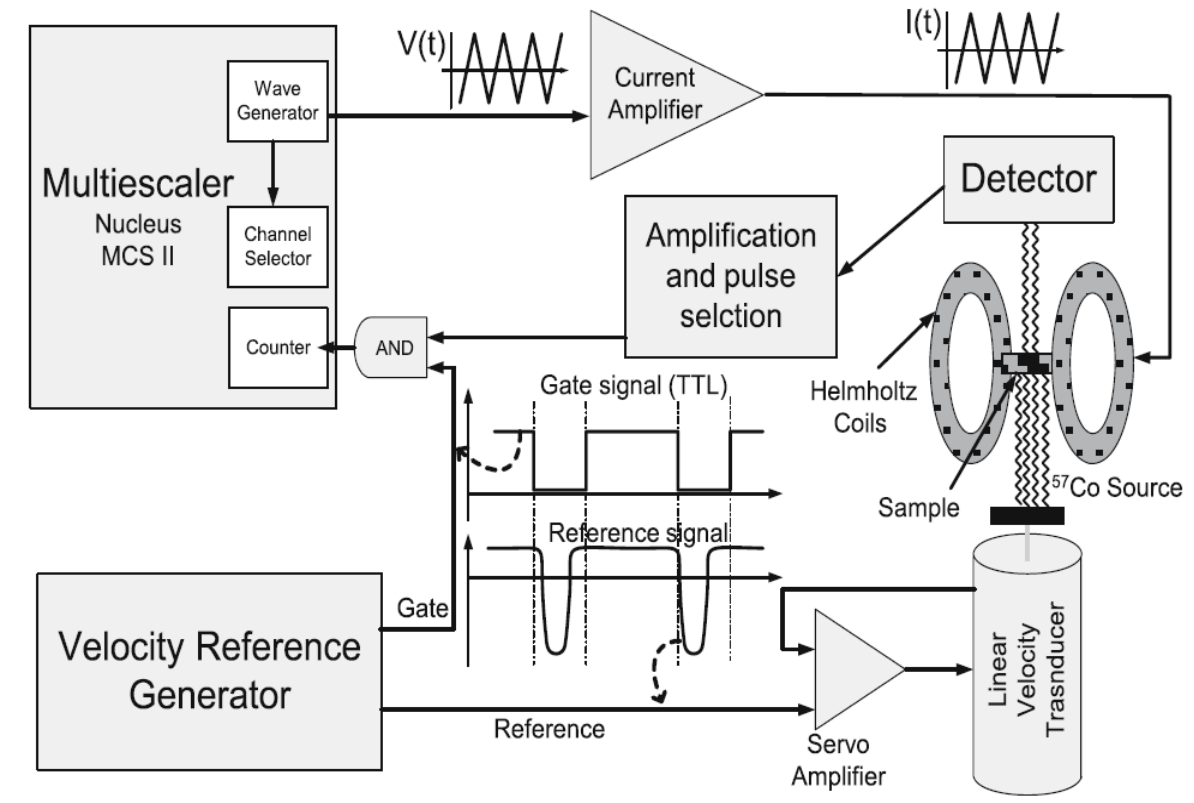

Figura 85: Disposición experimental para los experimentos de barrido de campo magnético [Pasquevich et al 2006] 
En la Figura 86 se muestra el diagrama esquemático del amplificador de corriente diseñado. Éste convierte la referencia triangular de tensión en corriente en el bobinado (proporcional al campo magnético). Para el sensado de la corriente se utilizó un resistor en serie con el bobinado, utilizando la tensión sobre éste como realimentación. Se utilizó el amplificador de potencia integrado LM4780 de National. Las alimentaciones de este amplificador se implementaron utilizando los reguladores ajustables con protecciones internas LM317HVK (positivo) y LM337HVK (negativo) de National Semiconductor (40 V, 1.5 A máximo).

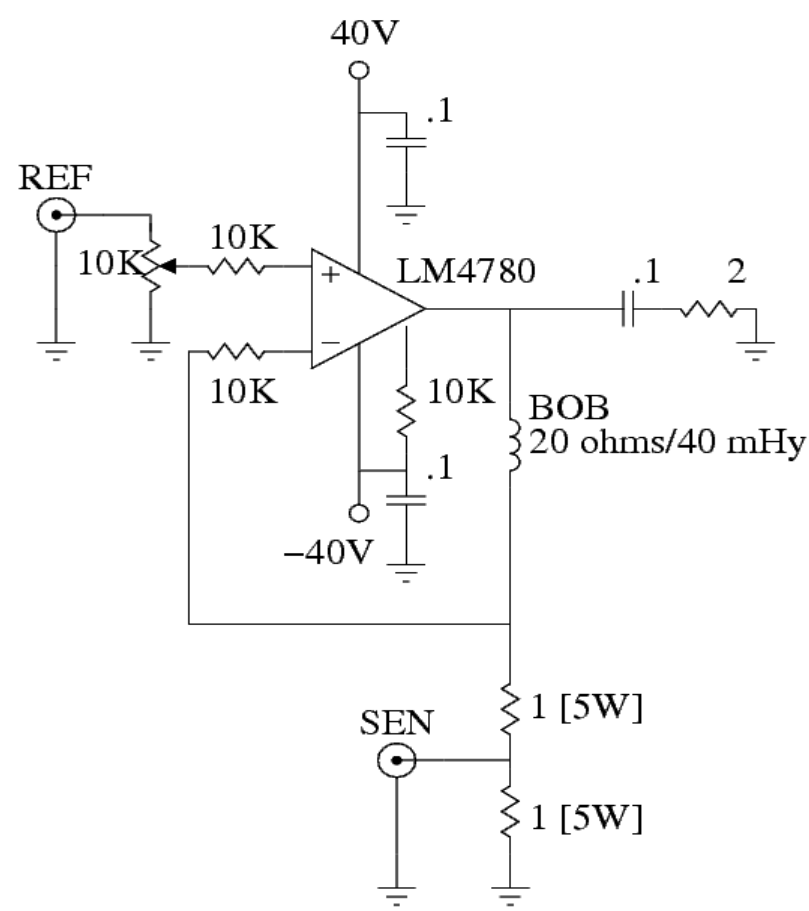

Figura 86: Esquema realimentado para control de corriente sobre un arrollamiento utilizando una referencia de tensión. 


\section{Apéndice $\mathbf{C}$}

\section{Control de velocidad con realimentación de posición}

A continuación se presenta la instrumentación diseñada para la operación de los transductores a desplazamiento constante, con realimentación discreta de la posición. Recordemos que en la Sección 4.3 se presentaron los fundamentos y los modos de operación de esta técnica. En la Figura 87 se presenta un diagrama funcional de la configuración experimental utilizada.

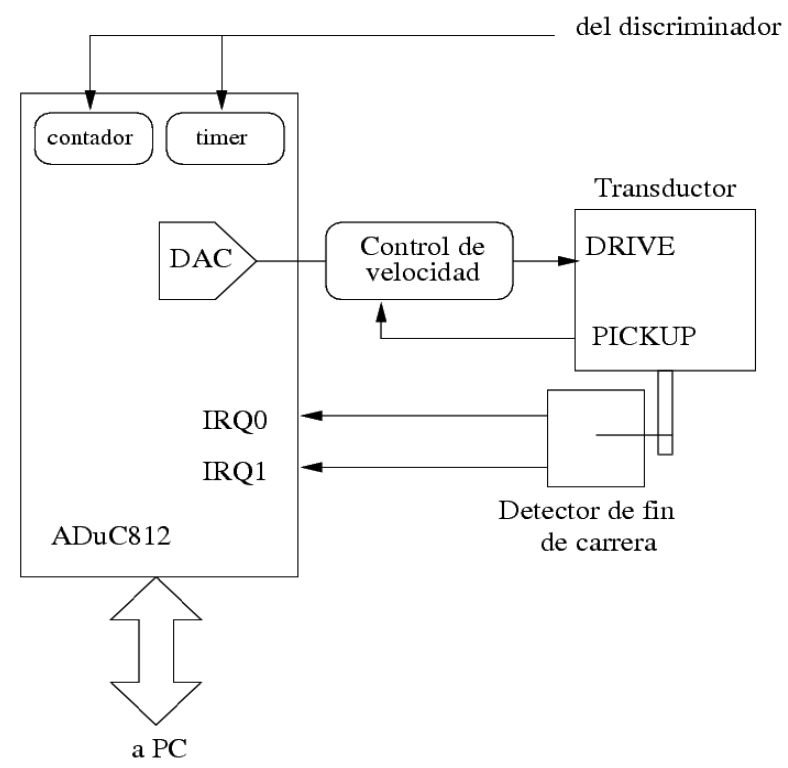

Figura 87: Diagrama en bloques de la configuración experimental.

El espectrómetro se compone de los siguientes bloques:

- Fuente de $\mathrm{Co}^{57}$

- Absorbente de hierro natural para las calibraciones. Espesor menor que el óptimo, lo que resulta en poco efecto (10\% esperado) y líneas bien angostas (ancho cercano al teórico).

- Etapa de detección idéntica a la utilizada en el Capítulo 6, optimizada para máxima relación señal a ruido.

- Etapa de accionamiento compuesta por:

i. Transductor ASA con una resistencia de $62 \Omega$ en serie, montado cuidadosamente para evitar las perturbaciones externas.

ii. Control de velocidad proporcional con realimentación unitaria, compensador serie y feedforward, acoplado en continua. Se utilizó un amplificador diseñado específicamente, con buena capacidad de tensión y corriente (Figura 88).

iii. Realimentación de posición discreta por fines de carrera, para accionamiento a desplazamiento constante, con un recorrido de aproximadamente $3 \mathrm{~mm}$, a una 
distancia grande a la fuente (Figura 89).

- Etapa de procesamiento diseñada para la aplicación, basada en el microcontrolador ADuC812 (versión previa al ADuC841 utilizado en versiones posteriores, con menor velocidad de procesamiento). El sensor de posición detecta los dos extremos de la trayectoria, indicando su ocurrencia con sendas interrupciones al microcontrolador. Ante estas señales, el programa conmuta entre la tensión positiva correspondiente a la velocidad deseada y la tensión negativa de retorno a máxima velocidad. El ratímetro y la inhibición del conteo se implementaron con recursos del microcontrolador. La etapa se montó en una placa con interfaz de 8 bits ISA para PC, utilizando comunicaciones basadas en interrupciones. El diagrama esquemático de la interfaz se presenta en la Figura 90. En la Figura 91 puede verse la configuración circuital hacia el exterior, en la cual se destaca el circuito de acondicionamiento de los pulsos por medio de un monoestable, y el acondicionamiento bipolar de la salida del conversor DA.

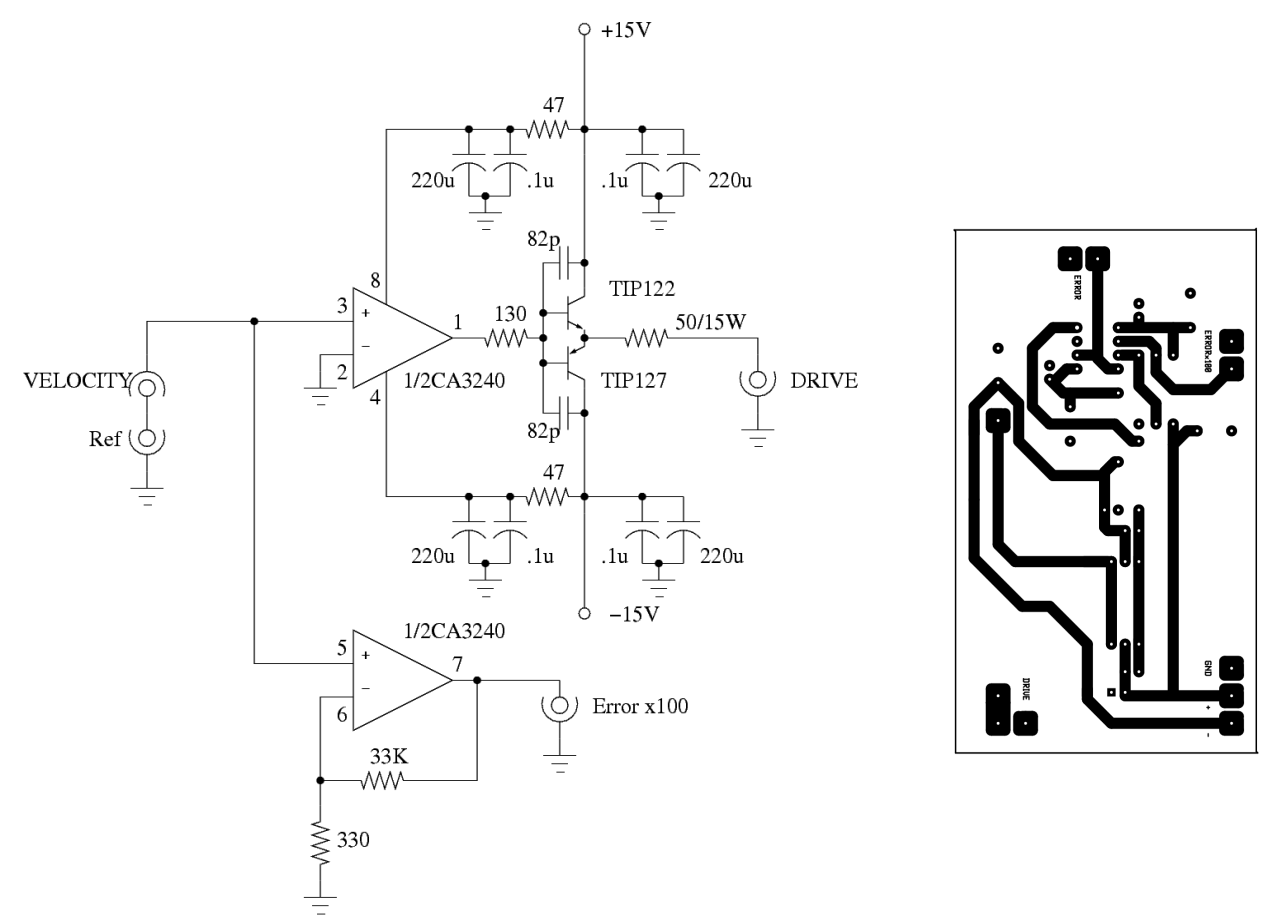

Figura 88: Diagrama esquemático y circuito impreso del control de velocidad acoplado en continua.
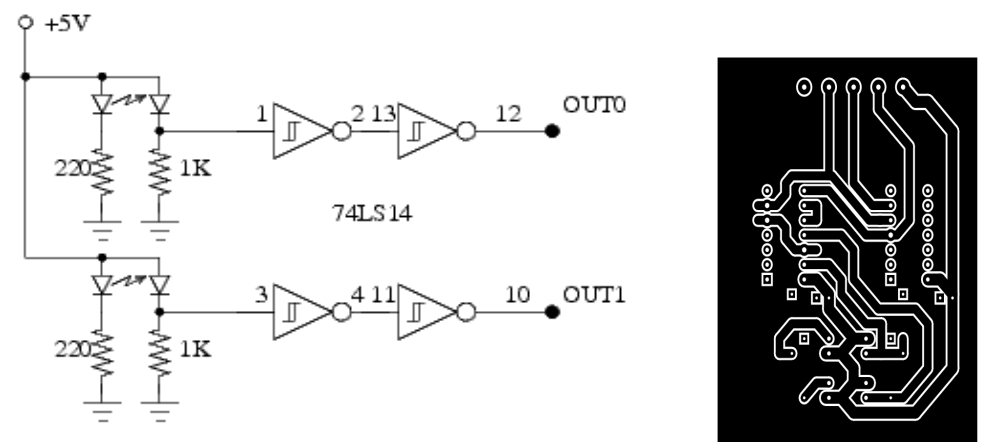

Figura 89: Diagrama esquemático y circuito impreso para el sensor de posición discreto. 


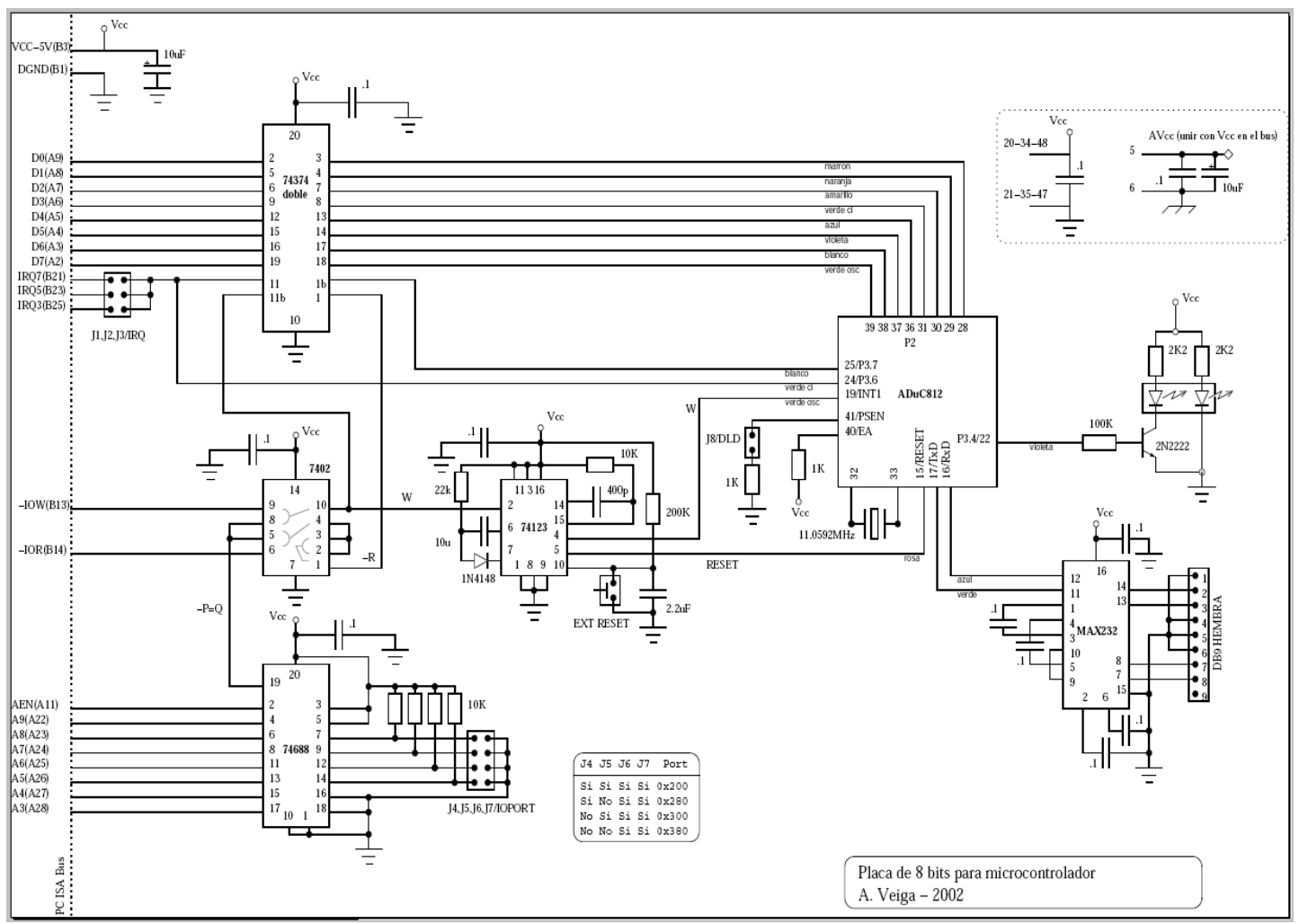

Figura 90: Diagrama esquemático de la placa ISA de 8 bits basada en el microcontrolador AduC812 que se utilizó como base para el diseño de la etapa de procesamiento.

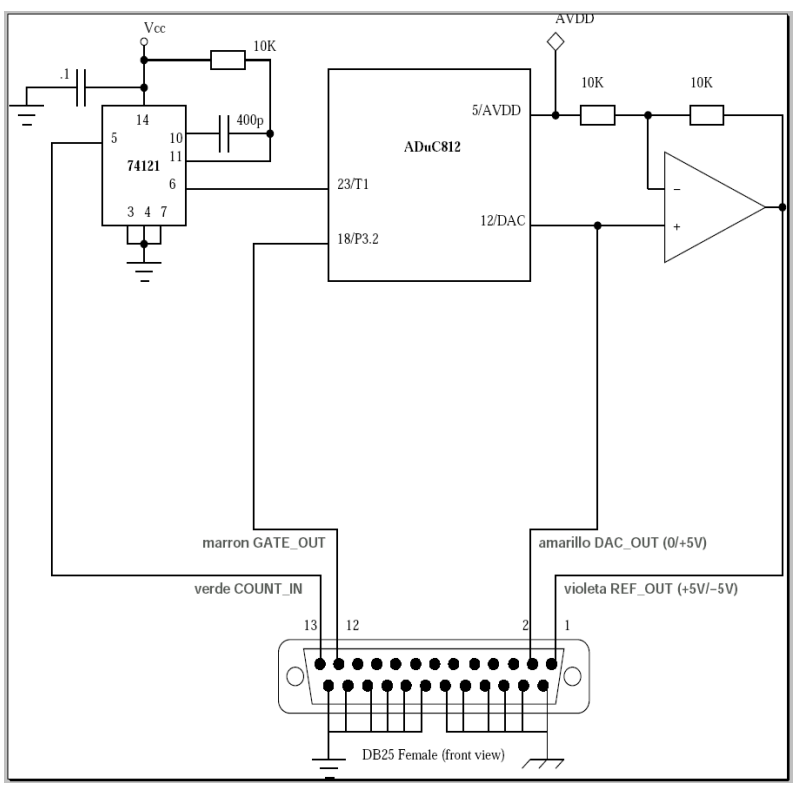

Figura 91: Entrada digital y salida analógica de la etapa de procesamiento. 
A fin de realizar una calibración del instrumento y observar su desempeño, se utilizó el espectrómetro para barrer el rango de velocidades que va desde el origen hasta los $6 \mathrm{~mm} / \mathrm{s}$, sólo velocidades positivas, utilizando el absorbente de calibración. Se midieron 120 canales en dos pasadas de 10 segundos netos por canal. En la Figura 92 puede verse uno de los espectros obtenidos en estas condiciones.

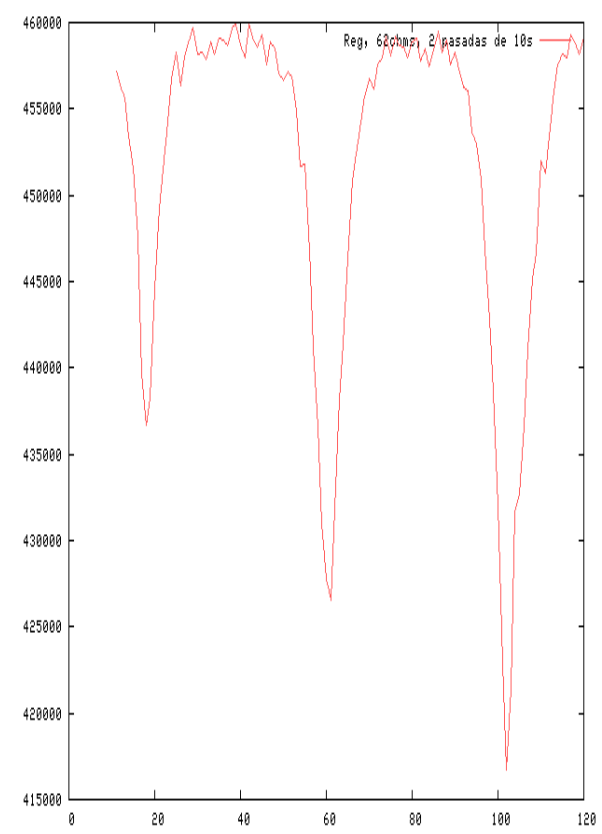

Figura 92: Espectro resultante de la calibración con hierro natural.

Respecto de la calidad del espectro pueden hacerse las siguientes observaciones:

Eficiencia: Resulta un duty-cycle de 0,6 para altas velocidades (pobre) y de casi 1 para bajas velocidades, propiedad innata del accionamiento a desplazamiento constante.

Resolución: Puede observarse un ancho de línea de aproximadamente $0.20 \mathrm{~mm} / \mathrm{s}$, lo cual es excelente en términos del control de velocidad.

Linealidad: La calibración de la abscisa, con los mínimos en los canales 18, 60 y 102 resulta satisfactoria $(60-18=42,102-60=42)$. Se observa un corrimiento de 5 canales a la derecha debido al offset de la etapa de accionamiento.

El control de velocidad presenta la limitación descripta a bajas velocidades y no posee buen comportamiento ante cambios grandes de velocidad, por lo que el espectro se tomó a canales adyacentes.

En conclusión, el espectro de calibración muestra un comportamiento aceptable del instrumento. La realimentación de posición discreta demostró ser un procedimiento sencillo y eficiente para auxiliar al sistema de control durante el accionamiento a velocidad constante programable. El diseño del instrumental asociado es lo suficientemente simple como para poder implementarlo a bajo costo en cualquier laboratorio de espectroscopia Mössbauer. 


\section{Apéndice D \\ Publicaciones relacionadas con la tesis}

\section{Tesis de Magister}

Sistemas jerárquicos de tiempo real para la adquisición de datos y control. Tesis de Magister en Ingeniería de la Universidad Nacional de La Plata. 1999, Facultad de Ingeniería. Director: Dr. Miguel Mayosky. Co-director: Nolberto Martínez.

\section{Artículos en revistas internacionales con referato}

Experimental design and methodology for a new Mössbauer scan experiment: absorption line tracking. A. Veiga, G.A. Pasquevich, P. Mendoza Zélis, F.H. Sánchez, M.B. Fernández van Raap and N. Martínez. Hyperfine Interactions (In Press).

Advances in constant-velocity Mössbauer instrumentation. A. Veiga, N. Martínez, P. Mendoza Zélis, G.A. Pasquevich and F.H. Sánchez. Hyperfine Interactions (2006) 167:905-909

Magnetic and thermal Mössbauer effect scans: a new approach. G.A. Pasquevich, P. Mendoza Zélis, F.H. Sánchez, M.B. Fernández van Raap, A. Veiga and N. Martínez. Hyperfine Interactions (2006) 167:839-844.

Determination of the iron atomic magnetic moments dynamics in the nanocrystalline ribbons Fe90Zr7B3 by Mössbauer magnetic scans. G.A. Pasquevich, P. Mendoza Zélis, F.H. Sánchez, M.B. Fernández van Raap, A. Veiga and N. Martínez. Physica B: Condensed Matter (2006) 384:348-350.

A constant velocity Mossbauer spectrometer with controlled temperature swep. $\underline{\text { A. }}$ Veiga, M.A. Mayosky, N. Martínez, E. Spinelli, P. Mendoza Zélis, G. Pasquevich and F. Sánchez. Review of Scientific Instruments (2002) 73:3579-3583.

A New Application of Mössbauer Effect Thermal Scans: Determination of the Magnetic Hiperfine Field Temperature Dependence. P. Mendoza Zélis, G. Pasquevich, F. Sánchez, N. Martínez and A. Veiga. Physics Letters A (2001) 289:55-59.

A hardware/software environment for real time data acquisition and control. A. Veiga, M.A. Mayosky and N. Martínez. IEEE Transactions on Nuclear Science (2000) 47:132-135.

\section{Presentaciones a congresos}

ICAME 2007, International Conference on the Applications of the Mössbauer Effect. IIT Kanpur, India. Octubre de 2007. 
Experimental design and methodology for a new Mössbauer scan experiment: absorption line tracking. A. Veiga, G.A. Pasquevich, P. Mendoza Zélis, F.H. Sánchez, M. B. Fernández van Raap and N. Martínez. Res. pág. T9-P18.

LACAME 2006, Latin American Conference on the Applications of the Mössbauer Effect. Rio de Janeiro, Brasil. Noviembre de 2006.

Mössbauer Magnetic Scans technique development. G.A. Pasquevich, P. Mendoza Zélis, M.B. Fernández van Raap, A. Veiga, N. Martínez, F.H. Sánchez. P044, abstracts p. 55.

HFILP 2005, Hyperfine Interactions International Workshop. La Plata, Noviembre de 2005. Tres trabajos presentados.

Advances in constant-velocity Mössbauer instrumentation. A. Veiga, N. Martínez, G.A. Pasquevich, F.H. Sánchez, P. Mendoza Zélis. Res. pág. 87.

Experimental design and quantitative analysis of Mössbauer scans. Present and future applications. F.H. Sánchez, G.A. Pasquevich, P. Mendoza Zélis, A. Veiga, N. Martínez. Res. Pág. 95.

Nanocrystallization kinetic of $\mathrm{Fe}_{90} \mathrm{Zr}_{7} \mathrm{~B}_{3}$ studied by Mössbauer isotermal scans. G.A. Pasquevich, F.H. Sánchez, P. Mendoza Zélis, M.B. Fernández van Raap y A. Veiga. Res. pág. 82.

90ª Reunión de la Asociación Física Argentina. La Plata, Septiembre de 2005. Tres trabajos presentados.

Control doble de temperatura con salidas acopladas. A. Veiga, N. Martínez y S.A. Verne. Res. pág. 194.

Etapa de potencia programable para un control de temperatura. S.A. Verne, A. Veiga y N. Martínez. Res. pág. 197.

Estudio de la dinámica de los momentos magnéticos del Fe en el nanocompuesto $\mathrm{Fe}_{90} \mathrm{Zr}_{7} \mathrm{~B}_{3}$ mediante transmisión Mössbauer a velocidad Doppler constante. G.A. Pasquevich, F.H. Sánchez, P. Mendoza Zélis, M.B. Fernández van Raap y A. Veiga. Res. pág. 169.

ICAME 2005, International Conference on the Applications of the Mössbauer Effect. Montpellier, Francia, Septiembre de 2005. Dos trabajos presentados.

Advances in constant-velocity Mössbauer instrumentation. A. Veiga, N. Martínez, G.A. Pasquevich, F.H. Sánchez, P. Mendoza Zélis. Res. pág. T6-P26.

Experimental design and quantitative analysis of Mössbauer scans. Present and future applications. F.H. Sánchez, G.A. Pasquevich, P. Mendoza Zélis, A. Veiga, N. Martínez.

86 $^{a}$ Reunión de la Asociación Física Argentina. Rosario, Septiembre de 2001.

Barrido Térmico del Efecto Mössbauer a Velocidad Constante: Deteminación de la dependencia con la Temperatura del campo Magnético Hiperfino. P. Mendoza Zélis, G. Pasquevich, F. Sánchez, N. Martínez, A. Veiga. 
$6^{\text {th }}$ IFAC Workshop on Algorithms and Architectures for Real Time Control. Palma de Mallorca, España. Mayo 2000.

Distributed Data Acquisition and Control using Embedded RTLinux Nodes: Performance and Perspectives. C. Grunfeld, A. Veiga, M.A. Mayosky y N. Martínez.

IEEE Real-Time Conference. New Mexico, USA. Junio 1999.

A hardware/software environment for real time data acquisition and control. A. Veiga, M.A. Mayosky y N. Martínez.

XVI Congreso Argentino de Control Automático. 1998, Buenos Aires, Argentina. Uso del Linux en la supervision y control de experimentos. N. Martínez, E. Spinelli y A. Veiga. Proceedings VOL II, pp567-572.

$8^{\circ}$ Congreso Latinoamericano de Control Automático. 1998, Viña del Mar, Chile. Uso del Linux en la supervision y control de experimentos. N. Martínez, E. Spinelli y A. Veiga. Proceedings VOL II, pp523-528.

III Congreso Argentino de Ciencias de la Computación (CACIC 97). La Plata, 1997. Microcontroladores en tiempo real. E. Spinelli y A. Veiga. Proceedings del Congreso, Vol 1, pag 96-113.

82 ${ }^{a}$ Reunión de la Asociación Física Argentina. 1997, San Luis, Argentina. Categoría: Física General, Ciencia de la Medición, Técnicas de Laboratorio e Instrumentación. Control y supervisión remota de experiencias de laboratorio. N. Martínez, E. Spinelli y $\underline{\text { A. Veiga. }}$.

81ª Reunión de la Asociación Física Argentina. 1996, Tandil, Argentina. Regulador de alta tensión para detectores proporcionales. N. Martínez, E. Spinelli y A. Veiga.

$7^{\circ}$ Congreso Latinoamericano de Control Automático. 1996, Buenos Aires, Argentina. Plataforma de bajo costo para implementación de algoritmos de control digital. $\mathrm{N}$. Martínez, E. Spinelli y A. Veiga. 


\section{Bibliografía}

Bhide V. G. (1973) Mössbauer Effect and its Applications, McGraw-Hill Publishing Co. Ltd.

Bracewell R. N. (1965) The uncertainty relation in The Fourier Transform and its Applications, Capítulo 8, p.160-163. McGraw-Hill 1965.

Cranshaw T. E., B.W. Dale, C.O. Longworth and C.E. Johnson (1985) Mössbauer Spectroscopy and its Applications, Cambridge Univ. Press.

Danon J., Gordon and Breach (1968) Lectures on the Mössbauer Effect, Science Publishers.

Dickson, D. and F. Berry (1986) Mössbauer Spectroscopy, Cambridge University Press.

EG \& G-Ortec Catalog, EG \& G-Ortec, PSD Division, 100 Midland Road, Oak Ridge, Tenn. 37380 USA.

Fraunfelder H. (1963) The Mössbauer Effect, W. A. Benjamin, New York.

Greenwood N.N. and T.C. Gibb (1971) Mössbauer Spectroscopy, Chapman and Hall, London.

Knoll G.F. (2000) Radiation Detection and Measurement, $3^{\text {rd }}$ Edition, John Willey \& Sons, New York.

Kuo B (1996) Sistemas de Control Automático, séptima ed. Prentice Hall.

Leo W.R. (1994) Techniques for Nuclear and Particle Physics Experiments, $2^{\text {nd }}$ Edition, Springer-Verlag, New York.

Lewis P. and C. Yang (1997) Sistemas de Control en Ingeniería. Prentice Hall.

Long G. J. and J.G. Stevens (1986) Industrial applications of the Mössbauer Effect, Plenum Press, New York.

Lurie B. and P. Enright (2000) Classical Feedback Control. Marcel Dekker Inc, NY.

Ogata K (1998) Ingeniería de Control Moderna. Prentice Hall.

Papoulis, A. (1962) Duration of a signal and uncertainty principle, en "The Fourier Integral and its Applications", Capítulo 4, p.62-74. McGraw-Hill, New York 1962.

Thosar B.V. and P.K. Iyengar, Eds. (1982) Advances in Mössbauer Spectroscopy, Elsevier, New York.

Vértes A., L. Korecz and K. Burger (1979) Mössbauer Spectroscopy, Elsevier Scientific Publishing Company.

Wertheim G. K. (1964) Mössbauer Effect: Principles and Applications, Academic Press. 


\section{Referencias}

Azevedo M.M.P. de, M.S. Rogalski and J.B. Sousa (1997) A user-friendly PC program for evaluation of Mössbauer spectra. Measusement Science and Technology 8941.

Baek, K.S., E.J. Hahn and H.N. Ok (1987) Mössbauer study of Ni0.01Fe0.99S. Physical Review B 361.

Battaiotto P., J.M. Catalfo, N. Martínez, M.A. Mayosky and G. Toccaceli (1994) A DSPbased Mossbauer Spectrometer. Proccedings of the 20th International Conference on Industrial Electronics, Control and and Instrumentation 31834.

Benedetti M. and D.R. Fernandez (1981) Fully programmable microprocessor-controlled Mössbauer spectrometer. Review of Scientific Instruments 521406.

Benedetti M. and N. Martínez (1982) Servosistema de control de velocidad para un transductor Mossbauer. Revista Telegráfica Electrónica 82766.

Blois J. de, A. J. H. Beunes, E. Boellaard, A. M. van der Kraan, E. A. Osterholt and M. N. Schipper (1998) A single-board VME mössbauer-spectrometry module. Nuclear Instruments and Methods in Physics Research Section B: Beam Interactions with Materials and Atoms 142173.

Carmeliet J. and S. Lejeune (1968) Electromechanical drive employing feedback for Mossbauer experiments. Nuclear Instruments and Methods 62166.

Casas Ll., E. Molins and A. Roig (2002) Miniaturization of a Mössbauer spectrometer using a piezotransducer and a solid state detector. Hyperfine Interactions 141125.

Chase R.L. (1969) Flyback system for a constant velocity Mössbauer drive. Review of Scientific Instruments 4085.

Chien C.E. (1978) Mössbauer study of a binary amorphous ferromagnet $\mathrm{Fe}_{80} \mathrm{~B}_{20}$. Physical Review B 181003.

Cohen R.L., P.G. McMullin and G.K. Wertheim (1963) High velocity drive for Mössbauer experiments. Review of Scientific Instruments 34671.

Cook D.C. (2004) Application of Mössbauer spectroscopy to the study of corrosion. Hyperfine Interactions 15361.

Corson M.R. (1980) Shape correction technique for stabilizing and linearizing a constant acceleration Mössbauer velocity drive. Review of Scientific Instruments 51331.

Cranshaw T.E. (1973) The problems associated with the interferometric calibration of Mössbauer spectrometer. Journal of Physics E: Scientific Instruments 61053.

Cranshaw T.E. (1974) Mössbauer Spectroscopy. Journal of Physics E: Scientific Instruments 
7497.

De Benedetti S. (1960) The Mössbauer Effect. Scientific American 20273.

De Souza P., B. Bernhardt, G. Klingerlhöfer and P. Gütlich (2003) Surface analysis in archelogy using the miniaturized Mössbauer spectrometer MIMOS II. Hyperfine Interactions 151125.

Ericson M.N, G.W. Turner, D.E. McMillan, B.S. Hoffheins, R.A. Todd and J.M. Hiller (1994) A Miniaturized ASIC-Based Multichannel Scaler Instrument. IEEE Transactions on Nuclear Science 411217.

Eymery J.P. and Teillet J. (2000) Spectrométrie Mössbauer, Techniques de l'Ingénieur, traité Analyse et Caractérisation.

Filter W.F., R.H. Sands and W.R. Dunham (1996) An ultra-high-stability Mössbauer spectrometer drive using a type-2 feedback system. Nuclear Instruments and Methods in Physics Research Section B: Beam Interactions with Materials and Atoms 119565.

Flinn P.A. (1963) Velocity servo drive for a high precision Mössbauer spectrometer. Review of Scientific Instruments 341422.

Gaitanis N., A. Kostikas and A. Simopoulos (1969) A constant acceleration Mossbauer spectrometer with velocity range selectivity. Nuclear Instruments and Methods 75274.

Genand-Riondet N., P. Imbert and J.F. Lericque (1978) Improved electromagnetic velocity drive for Mössbauer experiments. Review of Scientific Instruments 49606.

Gupta A.K. and K.R. Sarma (1973) An ultrastable Mössbauer spectrometer using integrated circuits. Review of Scientific Instruments 4514234.

Jing J., S.J. Campbell and J. Pellegrino (1992) A stand-alone Mössbauer spectrometer based on the MC68008 microprocessor. Measurement Sciece and Technology 380.

Kankeleit E. (1964) Velocity spectrometer for Mössbauer experiments. Review of Scientific Instruments 35194.

Klingelhöfer G. (2002) The miniaturized Mössbauer spectrometer MIMOS II for extraterrestrial and outdoor terrestrial applications: A status report. Hyperfine Interactions 144371.

Kluger A. and S.E. Enescu (2003) Mössbauer acquisition system for Rayleigh scattering experiments. Review of Scientific Instruments 743035.

Lang G. (1970) Mössbauer Spectroscopy of Haem Proteins. Quarterly Review of Biophysics 31.

Lierop van J. and D.H. Ryan (2001) An improved selective excitation double Mössbauer spectrometer. Review of Scientific Instruments 723349.

Lipkin J., B. Schechter, S. Shtrikman and D. Treves (1964) Inexpensive automatic recording 
Mössbauer spectrometer. Review of Scientific Instruments 351336.

Martínez N., E. Spinelli and A. Veiga (1996) Fuente de alimentación para contadores proporcionales. 81 ${ }^{\circ}$ Reunión Nacional de Física. Tandil, 16 al 20 de septiembre de 1996.

Mashlan M., D. Jancik, A. Kholmetskii, A. Mulaba and H. Pollak (1999) Mössbauer spectrometer with YAIO3:Ce scintillation crystal, Hyperfine Interactions 120769.

Mashlan M., A. Kholmetskii, V. Yevdokimov, J. Pechousek, O. Verich, R. Zboril and R. Tsonchev (2006) Mössbauer spectrometer with resonant detector. Nuclear Instruments and Methods in Physics Research Section B: Beam Interactions with Materials and Atoms 243 241.

Mendoza Zélis P., G. Pasquevich, F. Sánchez, N. Martínez and A. Veiga (2002) A New Application of Mössbauer Effect Thermal Scans: Determination of the Magnetic Hiperfine Field Temperature Dependence. Physics Letters A 28955.

Mendoza Zélis P., C. Rodriguez Torres, F.A. Cabrera, M. Fernández Van Raap, G. Pasquevich, F.H. Sánchez, A. González and J.J. Suñol. (2004) Thermal evolution of $\mathrm{Fe}_{65} \mathrm{Ni}_{20} \mathrm{Nb}_{6} \mathrm{~B}_{9}$ nanocrystalline metastable alloy. Journal of Metastable and Nanocrystalline Materials 20571.

Mössbauer R.L. (1958) Kernresonzflureszenz von Gammastrahlung in Ir191. Z. Physik 151 124.

Mössbauer R.L. (1961) Recoiless nuclear resonance absorption of gamma radiation. Nobel lecture. December 11, 1961.

Müller W.M. (1973) Dead-time problems. Nuclear Instruments and Methods 11247.

Nama H.D., E.C. Kimb and J.S. Hanb (2005) Mössbauer study of iron sulfides doped with 3d-transition metals. Solid State Communications 1355.

Overbeeke J. van (1971) A springless Mössbauer drive system. Journal of Physics E: Scientific Instruments 4549.

Pasquevich G.A., P. Mendoza Zélis, M.B. Fernández van Raap and F. H. Sánchez (2004) Hyperfine field temperature dependence of $\mathrm{Fe}_{3} \mathrm{Si}$ from Mössbauer thermal scans. Physica B 354369.

Pasquevich G.A., P. Mendoza Zélis, F.H. Sánchez, M.B. Fernández van Raap, A.Veiga and N. Martínez (2006) Magnetic and thermal Mössbauer effect scans: a new approach. Hyperfine Interactions 167839

Pasquevich G.A., P. Mendoza Zélis, F.H. Sánchez, M.B. Fernández van Raap, A. Veiga and N. Martínez (2006) Determination of the iron atomic magnetic moments dynamics in the nanocrystalline ribbons Fe90Zr7B3 by Mössbauer magnetic scans. Physica B: Condensed Matter 384348.

Player M.A. and F.W.D. Woodhams (1976) An improved interferometric calibrator for Mössbauer spectrometer drive systems. Journal of Physics E: Scientific Instruments 91148. 
Player M.A. and F.W.D. Woodhams (1978) A simple microprocessor Mössbauer spectrometer. Journal of Physics E: Scientific Instruments 11191.

Ponikvar D. (2002) Simple drive system for Mössbauer spectroscopy. Nuclear Instruments and Methods in Physics Research Section B: Beam Interactions with Materials and Atoms 187555.

Pourcell K.F. and M.P. Edwards (1985) State-of-the-art time-resolved emission Mössbauer spectrometer. Review of Scientific Instruments 56108.

Rubin D. (1962) Constant acceleration transducer employing negative feedback for use in Mössbauer experiments. Review of Scientific Instruments 331358.

Ruegg, F.C., J.J. Spijkerman and J.R. DeVoe (1965) Drift free Mössbauer spectrometer. Review of Scientific Instruments 36356.

Saccone F.D., C.E. Rodríguez Torres, G.A. Pasquevich, M.B. Fernández van Raap and F.H. Sánchez (2004) Crystallization kinetics of B-rich Mischmetal-Fe-B nanocomposite ribbons. Physica B 354237.

Sánchez F.H. , G.A. Pasquevich, P. Mendoza Zélis, A.F. Cabrera, L. Ying 'Feng and M. Vázquez (2004) Study of Magnetic Materials by Mössbauer Thermal Scans. Journal of Metastable and Nanocrystalline Materials 2239.

Sarma P.R., A.K. Sharma and K.C. Tripathi (1979) A constant velocity Mossbauer spectrometer free of long-term instrumental and radioactive decay drift. Nuclear Instruments and Methods 164591.

Seberini M. (1988) A constant velocity Mössbauer drive. Journal of Physics E: Scientific Instruments 21641.

Veiga A. (1999) Sistemas Jerárquicos de Tiempo Real para Adquisición de Datos y Control. Tesis de Magister en Ingeniería, UNLP.

Veiga A., M.A. Mayosky and N. Martínez (2000) A hardware/software environment for real time data acquisition and control. IEEE Transactions on Nuclear Science 47132.

Veiga A., M.A. Mayosky, N. Martínez, E. Spinelli, P. Mendoza Zélis, G. Pasquevich and F. Sánchez (2002) A constant velocity Mossbauer spectrometer with controlled temperature sweep. Review of Scientific Instruments 733579.

Veiga A., N. Martínez, P. Mendoza Zélis, G.A. Pasquevich and F.H. Sánchez (2006) Advances in constant-velocity Mössbauer instrumentation. Hyperfine Interactions 167905

Veiga A., G.A. Pasquevich, P. Mendoza Zélis, F.H. Sánchez, M.B. Fernández van Raap and N. Martínez (2008) Experimental design and methodology for a new Mössbauer scan experiment: absorption line tracking. Hyperfine Interactions (In Press).

Velázquez A. (2005) Design and construction of an autonomous control system for Mössbauer Spectrometry. Hyperfine Interactions 169200. 
Waard H. de (1965) Optical method for absolute velocity calibration of Mössbauer spectrometers. Review of Scientific Instruments 361728.

Woodhams F.W.D. (1967) A simple Mössbauer spectrometer. Journal of Scientific Instruments $\mathbf{4 4} 285$.

Woodhams F.W.D. and S.M. Reeder (1991) A transputer-based Mössbauer spectrometer. Measurement Science and Technology 2217.

Woodhams F.W.D. and M.A. Player (2001) A low-cost electrically programmable logic device controlled Mössbauer spectrometer. Measurement Science and Technology 12 N1.

Yoshimura T. (1977) Absolute velocity control of a Mössbauer spectrometer by utilizing a laser interferometer. Journal of Physics E: Scientific Instruments 10829.

Zane R. (1966) Mossbauer effect velocity drive linearized by shape correction. Nuclear Instruments and Methods $\mathbf{4 3} 333$. 